Supporting Information

for

\title{
Enantioselective Copper-Catalyzed Alkynylation of Benzylic C-H Bonds via Radical Relay
}

\begin{abstract}
Liang Fu, ${ }^{\mathrm{a}}$ Zhihan, Zhang, ${ }^{\mathrm{b}}$ Pinhong Chen, ${ }^{\mathrm{a}}$ Zhenyang Lin, ${ }^{* \mathrm{~b}}$ and Guosheng Liu* ${ }^{\mathrm{a}, \mathrm{c}}$
${ }^{a}$ State Key Laboratory of Organometallic Chemistry and Shanghai Hongkong Joint Laboratory in Chemical Synthesis, Center for Excellence in Molecular Synthesis, Shanghai Institute of Organic Chemistry, Chinese Academy of Sciences, 345 Lingling Road, Shanghai 200032, China. ${ }^{b}$ Department of Chemistry, The Hong Kong University of Science and Technology, Clear Water Bay, Kowloon, Hong Kong, China. ${ }^{c}$ Chang-Kung Chuang Institute, East China Normal University, 3663 North Zhongshan Road, Shanghai 200062, China.
\end{abstract}

Email: gliu@mail.sioc.ac.cn

Table of Contents

$\begin{array}{ll}\text { 1. General } & \text { S2 }\end{array}$

2. Synthesis and Characterization of Substrates and N-F Reagents $\quad$ S2

3. Optimization of the Reaction Conditions $\quad$ S5

4. General Procedure for the Asymmetric Alkynylation of Benzylic C-H Bonds $\quad$ S7

5. Late-Stage Functionalization and Synthetic Applications $\quad$ S7

$\begin{array}{ll}\text { 6. Synthetic Transformations } & \text { S10 }\end{array}$

$\begin{array}{ll}\text { 7. Mechanistic Studies } & \text { S13 }\end{array}$

$\begin{array}{lr}\text { 8. Large-Scale Reactions } & \text { S19 }\end{array}$

$\begin{array}{ll}\text { 9. Product Characterization } & \text { S21 }\end{array}$

10. Single Crystal X-Ray Diffraction Data (3s, 4d and 8) S44

$\begin{array}{ll}\text { 11. DFT Calculations } & \text { S69 }\end{array}$

12. References $\quad$ S82 


\section{General}

All commercially available compounds were purchased from Aldrich, Alfa Aesar or Adamas. NMR spectra was recorded on Bruker 400, Varian Inova 400 or Aglient $400\left(400 \mathrm{MHz}\right.$ for ${ }^{1} \mathrm{H}$; $376 \mathrm{MHz}$ for ${ }^{19} \mathrm{~F} ; 100 \mathrm{MHz}$ for ${ }^{13} \mathrm{C}$ ) spectrometer. The chemical shifts $(\delta)$ are given in parts per million relative to $\mathrm{CDCl}_{3}\left(7.26 \mathrm{ppm}\right.$ for $\left.{ }^{1} \mathrm{H}\right)$ and $\mathrm{CDCl}_{3}\left(77.0 \mathrm{ppm}\right.$ for $\left.{ }^{13} \mathrm{C}\right)$ and DMSO- $d_{6}$ $\left(2.50 \mathrm{ppm}\right.$ for $\left.{ }^{1} \mathrm{H}\right)$ and DMSO- $d_{6}\left(39.5 \mathrm{ppm}\right.$ for $\left.{ }^{13} \mathrm{C}\right)$. High performance liquid chromatography was performed on Waters 2487-600E, Thermo UltiMate 3000 or Waters ACQUITY UPC2, using AD-H, OD-H and OJ-H chiral columns eluted with a mixture of hexane and isopropyl alcohol (for $\mathrm{SFC}, \mathrm{CO}_{2}$ and $\mathrm{MeOH}$ were used as eluent). Optical rotation was measured on a Rudolph-Autopol I. Melting point was measured on SMP30. Flash column chromatography was performed on silica gel (particle size 200-300 mesh, purchased from Canada) and eluted with petroleum ether/ethyl acetate. Solvent was purified according to the procedure from a book named "Purification of Laboratory Chemicals". DMA and 1,2,4,5tetrafluorobenzene were deoxygenated with anhydrous Ar bubble for at least $30 \mathrm{~min}$ before they were used.

\section{Synthesis and Characterization of Substrates and N-F Reagents}

\subsection{Synthesis of N-F Reagents}

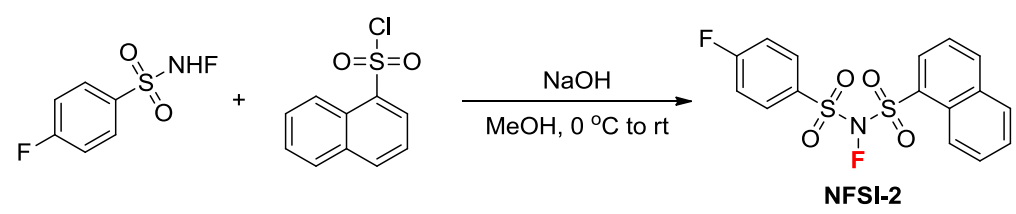

To a solution of $\mathrm{NaOH}(3.16 \mathrm{~g}, 79 \mathrm{mmol})$ in $\mathrm{MeOH}(80 \mathrm{~mL})$ was added $N$-fluoro-4fluorobenzenesulfonamide $(13.9 \mathrm{~g}, 72 \mathrm{mmol})$ at $0{ }^{\circ} \mathrm{C}$ under $\mathrm{N}_{2}$. After that, naphthalene-1sulfonyl chloride $(17.9 \mathrm{~g}, 79 \mathrm{mmol})$ was added to the homogenous solution in portions. The reaction was then warmed up to room temperature and stirred for 2 hours. The reaction mixture was filtered, the filter cake was crystallized from DCM to yield NFSI-2 (11.08 g, $40 \%$ yield).

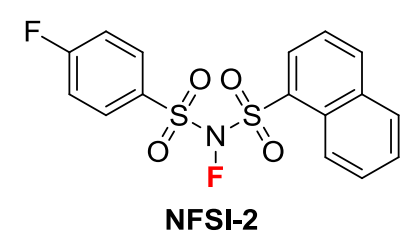

$N$-Fluoro- $N$-((4-fluorophenyl)sulfonyl)naphthalene-1-sulfonamide (NFSI-2): ${ }^{1} \mathrm{H}$ NMR (400 $\left.\mathrm{MHz}, \mathrm{CDCl}_{3}\right) \delta 8.75(\mathrm{~d}, J=8.4 \mathrm{~Hz}, 1 \mathrm{H}), 8.31-8.29(\mathrm{~m}, 1 \mathrm{H}), 8.25(\mathrm{~d}, J=8.0 \mathrm{~Hz}, 1 \mathrm{H}), 8.15-$ $8.11(\mathrm{~m}, 2 \mathrm{H}), 7.98(\mathrm{~d}, J=8.4 \mathrm{~Hz}, 1 \mathrm{H}), 7.81-7.77(\mathrm{~m}, 1 \mathrm{H}), 7.70-7.66(\mathrm{~m}, 1 \mathrm{H}), 7.60(\mathrm{t}, J=8.0$ $\mathrm{Hz}, 1 \mathrm{H}), 7.33-7.28(\mathrm{~m}, 2 \mathrm{H}) ;{ }^{13} \mathrm{C} \mathrm{NMR}\left(100 \mathrm{MHz}, \mathrm{CDCl}_{3}\right) \delta 167.14(\mathrm{~d}, J=258.6 \mathrm{~Hz}), 137.95$, 
134.15, 133.49, 133.14 (d, $J=9.4 \mathrm{~Hz}), 130.47$ (d, $J=2.8 \mathrm{~Hz}), 129.70,129.66,129.09,128.63$, 127.66, 124.46, 123.99, $116.94(\mathrm{~d}, J=23.0 \mathrm{~Hz}) ;{ }^{19} \mathrm{~F} \mathrm{NMR}\left(376 \mathrm{MHz}, \mathrm{CDCl}_{3}\right) \delta-36.06(\mathrm{~s}, 1 \mathrm{~F})$, -98.72-98.79 (m, 1F); IR (neat): $v=3108,1587,1488,1409,1373,1240,1191,1174,1084$, 786, 762, 673, 582, 550, 496; Anal. calcd for $\mathrm{C}_{16} \mathrm{H}_{11} \mathrm{~F}_{2} \mathrm{NO}_{4} \mathrm{~S}_{2}$ : C 50.13, H 2.89, N 3.65, found: C 50.08, H 3.13, N 3.63; melting point: $144-146{ }^{\circ} \mathrm{C}$.<smiles>O=S(=O)(c1ccc(F)cc1)N(F)S(=O)(=O)c1c(Cl)cccc1Cl</smiles>

2,6-Dichloro- $N$-fluoro- $N$-((4-fluorophenyl)sulfonyl)benzenesulfonamide (NFSI-1): ${ }^{1} \mathrm{H}$ NMR $\left(400 \mathrm{MHz}, \mathrm{CDCl}_{3}\right) \delta 8.18-8.14(\mathrm{~m}, 2 \mathrm{H}), 7.56-7.49(\mathrm{~m}, 3 \mathrm{H}), 7.36-7.32(\mathrm{~m}, 2 \mathrm{H}) ;{ }^{13} \mathrm{C} \mathrm{NMR}$ $\left(100 \mathrm{MHz}, \mathrm{CDCl}_{3}\right) \delta 167.33(\mathrm{~d}, J=259.3 \mathrm{~Hz}), 138.06(\mathrm{~d}, J=3.6 \mathrm{~Hz}), 135.53,135.47,133.36$ $(\mathrm{d}, J=10.0 \mathrm{~Hz}), 132.07,132.06,117.12(\mathrm{~d}, J=22.9 \mathrm{~Hz}) ;{ }^{19} \mathrm{~F}$ NMR $\left(376 \mathrm{MHz}, \mathrm{CDCl}_{3}\right) \delta-$ 36.71 (s, 1F), -98.23-98.30 (m, 1F); IR (neat): $v=3088,1586,1559,1489,1431,1404,1395$, $1245,1197,1185,1158,1086,853,784,676,590,543$; Anal. calcd for $\mathrm{C}_{12} \mathrm{H}_{7} \mathrm{Cl}_{2} \mathrm{~F}_{2} \mathrm{NO}_{4} \mathrm{~S}_{2}$ : C $35.84, \mathrm{H} 1.75, \mathrm{~N} 3.48$, found: C 35.95, H 1.86, N 3.61; melting point: $121-123{ }^{\circ} \mathrm{C}$.

\subsection{Effect of N-F Reagents on the Reaction}

Scheme S1. The effect of N-fluoroarenesulfonimides

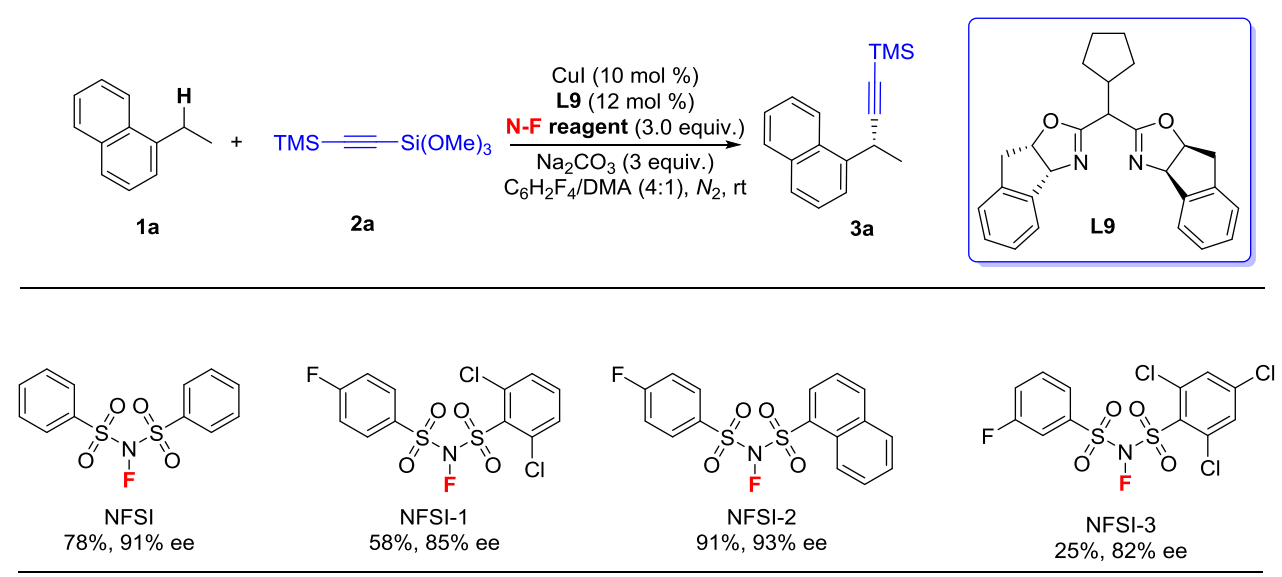

\subsection{Synthesis of Alkylarenes}
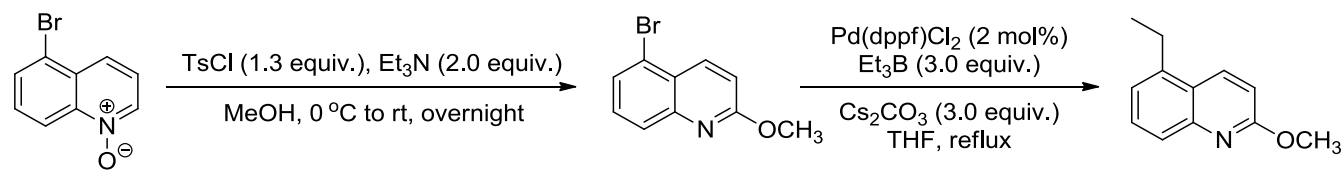

5-Bromo-2-methoxyquinoline was synthesized according to the literature. ${ }^{\mathrm{S} 1}$ To an icecooled solution of 5-bromoquinoline 1-oxide (3.36 g, $15 \mathrm{mmol})$ and $\mathrm{TsCl}$ (3.8 g, $20 \mathrm{mmol})$ in 
$\mathrm{MeOH}(90 \mathrm{~mL}), \mathrm{Et}_{3} \mathrm{~N}(4.2 \mathrm{~mL}, 30 \mathrm{mmol})$ was added at $0{ }^{\circ} \mathrm{C}$. The reaction mixture was then stirred at room temperature overnight. After reaction completion, $\mathrm{MeOH}$ was removed under vacuum, and the residue was quenched by $10 \% \mathrm{HCl}$. Then $\mathrm{HCl}$ solution was neutralized with aq. $\mathrm{Na}_{2} \mathrm{CO}_{3}$ and extracted with $\mathrm{CHCl}_{3}$. The combined organic phase was dried over $\mathrm{Na}_{2} \mathrm{SO}_{4}$ and concentrated under vacuum. The residue was then purified by column chromatography on silica gel $(\mathrm{PE} / \mathrm{EA}=20: 1)$ to yield 5-bromo-2-methoxyquinoline (2.49 g, 70\% yield) as a white solid.

5-Ethyl-2-methoxyquinoline was prepared according to the literature. ${ }^{\mathrm{S} 2}$ To a solution of $\mathrm{Pd}(\mathrm{dppf}) \mathrm{Cl}_{2} \quad(146 \mathrm{mg}, \quad 0.2 \mathrm{mmol}), \mathrm{Cs}_{2} \mathrm{CO}_{3} \quad(9.77 \mathrm{~g}, \quad 30 \mathrm{mmol})$ and 5-bromo-2methoxyquinoline $(2.38 \mathrm{~g}, 10 \mathrm{mmol})$ in dried THF $(30 \mathrm{~mL}), \mathrm{BEt}_{3}(30 \mathrm{~mL}, 30 \mathrm{mmol}, 1.0 \mathrm{M}$ in THF) was added under $\mathrm{N}_{2}$. Then, the mixture was heated up to reflux and monitored by TLC. After cooling down to room temperature, the mxiture was filtered through a short pad of celite, and the filtrate was concentrated under vacuum. The residue was purified by column chromatography on silica gel $(\mathrm{PE} / \mathrm{EA}=20: 1)$ to yield 5-ethyl-2-methoxyquinoline $(1.81 \mathrm{~g}$, $97 \%$ yield) as colorless oil.

${ }^{1} \mathrm{H}$ NMR $\left(400 \mathrm{MHz}, \mathrm{CDCl}_{3}\right) \delta 8.19(\mathrm{~d}, J=9.2 \mathrm{~Hz}, 1 \mathrm{H}), 7.71(\mathrm{~d}, J=8.4 \mathrm{~Hz}, 1 \mathrm{H}), 7.53(\mathrm{t}, J=$ $8.0 \mathrm{~Hz}, 1 \mathrm{H}), 7.21(\mathrm{~d}, J=7.2 \mathrm{~Hz}, 1 \mathrm{H}), 6.90(\mathrm{~d}, J=9.2 \mathrm{~Hz}, 1 \mathrm{H}), 4.07$ (s, 3H), 3.02 (q, $J=7.6$ $\mathrm{Hz}, 2 \mathrm{H}), 1.33(\mathrm{t}, J=7.6 \mathrm{~Hz}, 3 \mathrm{H}) ;{ }^{13} \mathrm{C} \mathrm{NMR}\left(100 \mathrm{MHz}, \mathrm{CDCl}_{3}\right) \delta 161.92,147.08,140.63$, $134.80,129.31,125.54,123.33,123.07,112.37,53.25,25.49,15.34$; IR (neat): $v=2966$, 2943, 2877, 1609, 1579, 1505, 1400, 1321, 1270, 1241, 1027, 808, 763, 716, 446, $432 \mathrm{~cm}^{-1}$; HRMS: m/z (ESI) calculated for $\left(\mathrm{C}_{12} \mathrm{H}_{14} \mathrm{NO}\right)[\mathrm{M}+\mathrm{H}]^{+}: 188.1070$, found: 188.1068 .

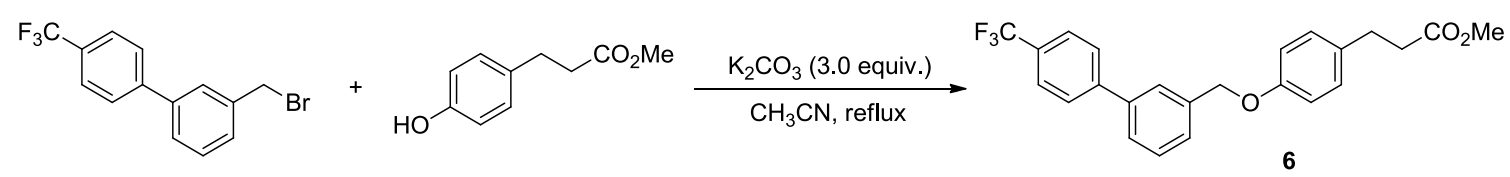

To a solution of 3-(bromomethyl)-4'-(trifluoromethyl)-1,1'-biphenyl (1.74 g, $5.5 \mathrm{mmol})$ and methyl 3-(4-hydroxyphenyl)propanoate $(0.9 \mathrm{~g}, 5 \mathrm{mmol})$ in $\mathrm{CH}_{3} \mathrm{CN}(20 \mathrm{~mL}), \mathrm{K}_{2} \mathrm{CO}_{3}(2.07$ $\mathrm{g}, 15 \mathrm{mmol}$ ) was added. The mixture was then heated up to reflux for 6 hours. After cooling down to room temperature, $\mathrm{CH}_{3} \mathrm{CN}$ was removed under vacuum and the residue was purified by column chromatography on silica gel (PE/EA = from 40:1 to 15:1) to yield methyl 3-(4((4'-(trifluoromethyl)-[1,1'-biphenyl]-3-yl)methoxy)phenyl)propanoate 6 (1.85 g, 89\% yield) as a white solid.

${ }^{1} \mathrm{H}$ NMR (400 MHz, $\left.\mathrm{CDCl}_{3}\right) \delta 7.69(\mathrm{~s}, 4 \mathrm{H}), 7.66(\mathrm{~s}, 1 \mathrm{H}), 7.55(\mathrm{~d}, J=6.8 \mathrm{~Hz}, 1 \mathrm{H}), 7.51-7.45$ $(\mathrm{m}, 2 \mathrm{H}), 7.13(\mathrm{~d}, J=7.6 \mathrm{~Hz}, 2 \mathrm{H}), 6.93(\mathrm{~d}, J=7.2 \mathrm{~Hz}, 2 \mathrm{H}), 5.10(\mathrm{~s}, 2 \mathrm{H}), 3.66(\mathrm{~s}, 3 \mathrm{H}), 2.90$ (t, $J=8.0 \mathrm{~Hz}, 2 \mathrm{H}), 2.60(\mathrm{t}, J=8.0 \mathrm{~Hz}, 2 \mathrm{H}) ;{ }^{13} \mathrm{C} \mathrm{NMR}\left(100 \mathrm{MHz}, \mathrm{CDCl}_{3}\right) \delta 173.31,157.16$, 144.36, 140.03, 137.96, 133.05, 129.39 (q, $J=32.3 \mathrm{~Hz}$ ), 129.27, 129.18, 127.41, 127.18, 126.79, 126.26, $125.65(\mathrm{q}, J=4.0 \mathrm{~Hz}), 124.24(\mathrm{q}, J=270.7 \mathrm{~Hz}), 114.83,69.79,51.50,35.86$, 30.04; ${ }^{19} \mathrm{~F}$ NMR (376 MHz, $\left.\mathrm{CDCl}_{3}\right) \delta-62.42$ (s, 3F); IR (neat): $v=2951,1733,1613,1583$, 1322, 1238, 1163, 1110, 1068, 1014, 810, 783, 699, 518, $429 \mathrm{~cm}^{-1}$; HRMS: m/z (EI) calculated for $\left(\mathrm{C}_{24} \mathrm{H}_{21} \mathrm{~F}_{3} \mathrm{O}_{3}\right)[\mathrm{M}]^{+}: 414.1437$, found: 414.1445 ; melting point: $62-64{ }^{\circ} \mathrm{C}$. 


\section{Optimization of the Reaction Conditions}

Table S1. Evaluation of copper catalysts., ${ }^{a, b}$

\begin{tabular}{|c|c|c|c|c|}
\hline \multirow[b]{3}{*}{ Entry } & \multirow{3}{*}{$\begin{array}{c}+\mathrm{TMS}=\mathrm{Si} \\
\mathbf{2 a} \\
\text { Copper catalyst }\end{array}$} & \multirow{2}{*}{\multicolumn{2}{|c|}{ 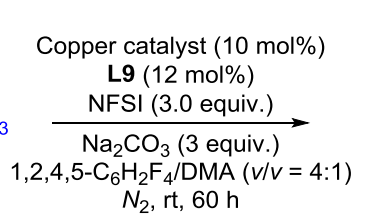 }} & \multirow{3}{*}{$\underbrace{e^{c}}_{3 a}$} \\
\hline & & & & \\
\hline & & Conv. $(\mathbf{1} \mathbf{a})^{b}$ & Yield of $\mathbf{3} \mathbf{a}^{b}$ & \\
\hline 1 & CuOAc & $84 \%$ & $75 \%$ & $91 \%$ \\
\hline 2 & Cul & $84 \%$ & $78 \%$ & $91 \%$ \\
\hline 3 & {$[\mathrm{CuOTf}]_{2} \mathrm{PhH}$} & $75 \%$ & $75 \%$ & $89 \%$ \\
\hline 4 & $\mathrm{Cu}\left(\mathrm{CH}_{3} \mathrm{CN}\right)_{4} \mathrm{PF}_{6}$ & $73 \%$ & $70 \%$ & $84 \%$ \\
\hline 5 & $\mathrm{Cu}\left(\mathrm{CH}_{3} \mathrm{CN}\right)_{4} \mathrm{BF}_{4}$ & $70 \%$ & $63 \%$ & $87 \%$ \\
\hline 6 & $\mathrm{Cu}\left(\mathrm{CH}_{3} \mathrm{CN}\right)_{4} \mathrm{OTf}$ & $74 \%$ & $72 \%$ & $87 \%$ \\
\hline 7 & CuTc & $84 \%$ & $74 \%$ & $91 \%$ \\
\hline 8 & $\mathrm{Cu}(\mathrm{OAc})_{2}$ & $86 \%$ & $69 \%$ & $91 \%$ \\
\hline 9 & $\mathrm{Cu}(\mathrm{OTf})_{2}$ & $69 \%$ & $69 \%$ & $87 \%$ \\
\hline
\end{tabular}

${ }^{a}$ Reaction conditions: copper catalyst (10 mol\%), L9 (12 mol\%), 1a $(0.2 \mathrm{mmmol}), 2 a(0.8 \mathrm{mmol}), \mathrm{NFSI}(0.6 \mathrm{mmol})$ and $\mathrm{Na}_{2} \mathrm{CO}_{3}(0.6 \mathrm{mmol})$ in $1,2,4,5-\mathrm{C}_{6} \mathrm{H}_{2} \mathrm{~F}_{4} / \mathrm{DMA}(2.5 \mathrm{~mL}, v / v=4: 1)$ under $N_{2}$ at rt for $60 \mathrm{~h} .{ }^{b}{ }^{1} \mathrm{H}$ NMR yield and conversion were determined by using $\mathrm{CH}_{2} \mathrm{Br}_{2}$ as an internal standard. ${ }^{c}$ ee value was determined by HPLC on a chiral stationary phase.

Table S2. Evaluation of solvents. ${ }^{a, b}$

A) Solvent screening.
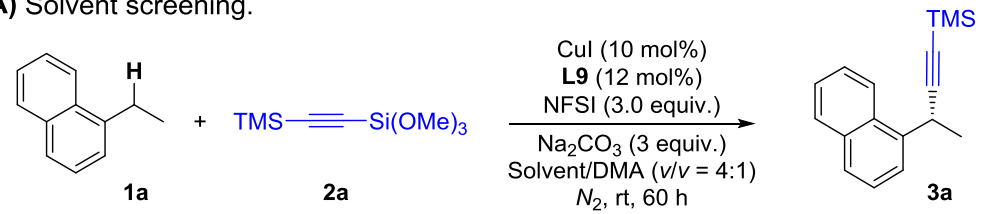

\begin{tabular}{|c|c|c|c|c|}
\hline Entry & Solvent & Conv. $(\mathbf{1} \mathbf{a})^{b}$ & Yield of $\mathbf{3} \mathbf{a}^{b}$ & $\mathrm{ee}^{c}$ \\
\hline 1 & $\mathrm{C}_{6} \mathrm{H}_{6}$ & $73 \%$ & $66 \%$ & $91 \%$ \\
\hline 2 & $\mathrm{Ph}-\mathrm{Cl}$ & $78 \%$ & $71 \%$ & $89 \%$ \\
\hline 3 & $\mathrm{Ph}-\mathrm{CH}_{3}$ & $67 \%$ & $61 \%$ & $90 \%$ \\
\hline 4 & $\mathrm{Ph}-\mathrm{CF}_{3}$ & $78 \%$ & $65 \%$ & $83 \%$ \\
\hline 5 & $\mathrm{Ph}-\mathrm{F}$ & $84 \%$ & $65 \%$ & $89 \%$ \\
\hline 6 & $1,2-\mathrm{C}_{6} \mathrm{H}_{4} \mathrm{~F}_{2}$ & $83 \%$ & $70 \%$ & $82 \%$ \\
\hline 7 & $1,2,3,4-\mathrm{C}_{6} \mathrm{H}_{2} \mathrm{~F}_{4}$ & $77 \%$ & $68 \%$ & $76 \%$ \\
\hline 8 & $1,2,4,5-C_{6} H_{2} F_{4}$ & $84 \%$ & $78 \%$ & $91 \%$ \\
\hline 9 & $\mathrm{C}_{6} \mathrm{~F}_{6}$ & $53 \%$ & $55 \%$ & $91 \%$ \\
\hline 10 & $1,4-\mathrm{C}_{6} \mathrm{H}_{4}-\left(\mathrm{CH}_{3}\right)_{2}$ & $47 \%$ & $42 \%$ & $89 \%$ \\
\hline $11^{d}$ & $\mathrm{C}_{6} \mathrm{~F}_{6}$ & $84 \%$ & $31 \%$ & $99 \%$ \\
\hline
\end{tabular}

${ }^{a}$ Reaction conditions: Cul (10 mol\%), L9 (12 mol\%), 1a $(0.2 \mathrm{mmmol}), \mathbf{2 a}(0.8 \mathrm{mmol})$, NFSI $(0.6 \mathrm{mmol})$ and $\mathrm{Na}_{2} \mathrm{CO}_{3}(0.6 \mathrm{mmol})$ in solvent/DMA $(2.5 \mathrm{~mL}, v / v=4: 1)$ under $\mathrm{N}_{2}$ at $\mathrm{rt}$ for $60 \mathrm{~h} .{ }^{b}{ }^{1} \mathrm{H}$ NMR yield and conversion were determined by using $\mathrm{CH}_{2} \mathrm{Br}_{2}$ as an internal standard. ${ }^{c}$ ee value was determined by HPLC on a chiral stationary phase. ${ }^{d}$ Pure $\mathrm{C}_{6} \mathrm{~F}_{6}(2 \mathrm{~mL})$ was used. 
B) The effect of DMAc and Box ligands on the asymmetric reaction.
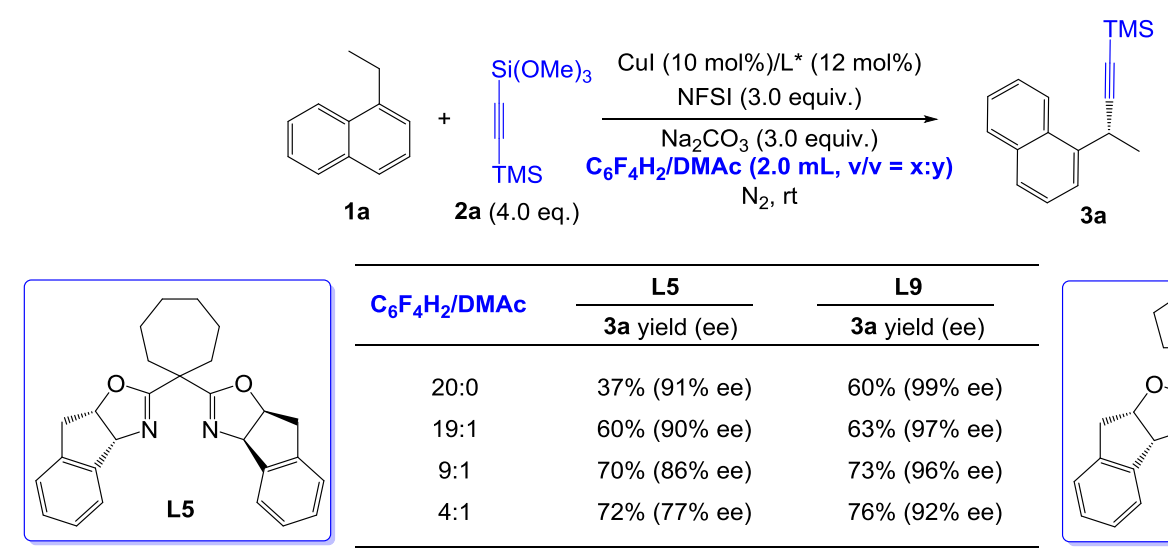

\begin{tabular}{|c|c|c|}
\hline \multirow{2}{*}{$\mathrm{C}_{6} \mathrm{~F}_{4} \mathrm{H}_{2} / \mathrm{DMAc}$} & L5 & L9 \\
\hline & 3a yield (ee) & 3a yield (ee) \\
\hline $20: 0$ & $37 \%$ (91\% ee) & $60 \%(99 \%$ ee $)$ \\
\hline $19: 1$ & $60 \%(90 \%$ ee $)$ & $63 \%(97 \%$ ee $)$ \\
\hline $9: 1$ & $70 \%$ ( $86 \%$ ee) & $73 \%(96 \%$ ee $)$ \\
\hline $4: 1$ & $72 \%(77 \%$ ee $)$ & $76 \%(92 \%$ ee $)$ \\
\hline
\end{tabular}

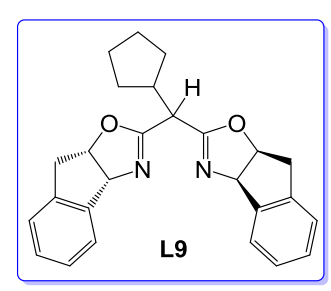

The experimental results in Figure S2-B suggested that the covalent complex is less likely involved in the catalytic reaction, and more elaborations are given as follows:

(1) During the ligand screening (Table S2-B), we found that the ligand $\mathbf{L 5}$ without gem-C-H bonds also exhibited excellent enantioselectivity (up to $91 \%$ ee) as L9 (up to 99\% ee). In addition, DMAc as co-solvent played an essential role in yields and enantioselectivities of the alkynylation products. Increasing the amount of DMAc could lead to better yields, but lower enantioselectivities; notably, a similar trend towards the DMAc effect on the reaction using L5 or L9 was observed (see Table S2B).

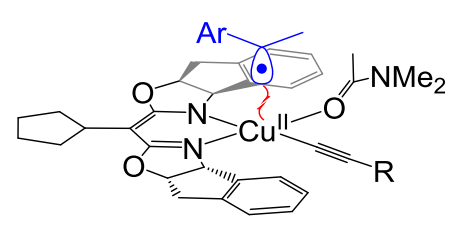

complex A

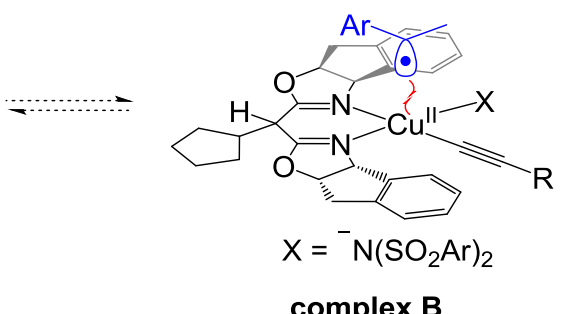

complex B

(eq S1)

(2) For L9, if a covalent $\mathrm{Cu}$ (II) complex $\mathbf{A}$ (eq S1) was involved in the radical trapping step, a neutral solvent molecule such as DMAc, instead of an anionic ligand, should coordinate to the $\mathrm{Cu}$ (II) center. However, the excellent enantioselectivity (up to $99 \%$ ee) was obtained in the absence of DMAc as co-solvent (see Table S2-B), and the ee values were decreased by increasing the concentration of DMAc, which are difficult to be addressed by the model of a covalent $\mathrm{Cu}(\mathrm{II})$ complex $\mathbf{A}$ (eq S1). In addition, the results presented in the manuscript showed that different N-F reagents could slightly change the ee values (see Table 1c, also see Scheme S1), suggesting that the non-covalent $\mathrm{Cu}$ (II) complex $\mathbf{B}$ containing the anion of bisarenesulfonamide (eq S1) is more likely involved. In view of the effect of N-F reagents on the ee values, we employed an anionic bisarylsulfonamide ligand instead of fluoride for our DFT mechanistic calculations (vide infra). As shown in Figure 2, the difference in the computed activation free energy $\left(\Delta \Delta \mathrm{G}^{\ddagger}\right)$ for the benzylic radical trapping by chiral $\mathrm{Cu}$ (II) species with a bismethylsulfonimide anion $(2.6 \mathrm{kcal} / \mathrm{mol})$ is pretty consistent with the 
experimental result observed in the absence of DMAc (99\% ee in Table S2-B), which also strongly support this mode.

Collected all, the additional experimental results suggest that a covalent complex is less likely involved in our reaction. However, we have to mention that it is difficult to explain the effect of co-solvent DMAc on yields and enantioselectivity at the moment.

Table S3. Evaluation of inorganic bases. ${ }^{a, b}$

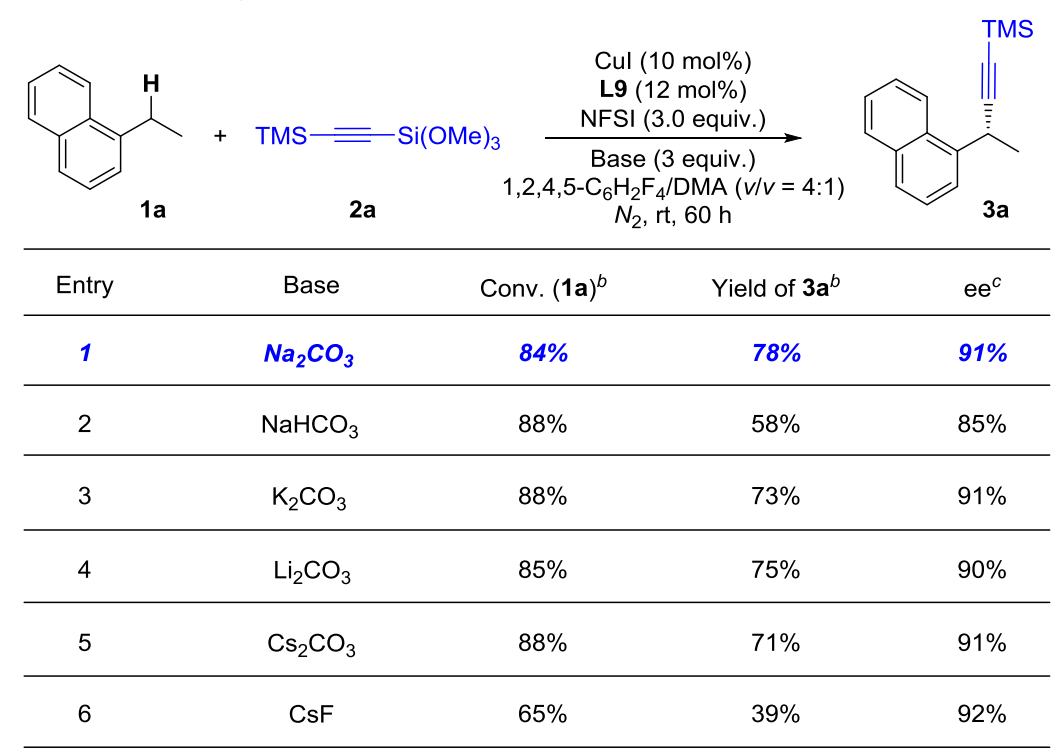

${ }^{a}$ Reaction conditions: Cul (10 mol\%), L9 (12 mol\%), 1a $(0.2 \mathrm{mmmol}), 2 \mathrm{a}(0.8 \mathrm{mmol}), \mathrm{NFSI}(0.6 \mathrm{mmol})$ and base $(0.6 \mathrm{mmol})$ in $1,2,4,5-\mathrm{C}_{6} \mathrm{H}_{2} \mathrm{~F}_{4} / \mathrm{DMA}(2.5 \mathrm{~mL}, v / v=4: 1)$ under $N_{2}$ at $\mathrm{rt}$ for $60 \mathrm{~h} .{ }^{b 1} \mathrm{H}$ NMR yield and conversion were determined by using $\mathrm{CH}_{2} \mathrm{Br}_{2}$ as an internal standard. ${ }^{c}$ ee value was determined by HPLC on a chiral stationary phase.

\section{General Procedure for the Asymmetric Alkynylation of Benzylic C-H Bonds}

In a dried sealed $10 \mathrm{~mL}$ Schlenk tube, $\mathbf{L 9}(9.6 \mathrm{mg}, 0.024 \mathrm{mmol}, 12 \mathrm{~mol} \%), \mathrm{CuI}(3.8 \mathrm{mg}$, $0.02 \mathrm{mmol}, 10 \mathrm{~mol} \%$ ) and anhydrous $\mathrm{Na}_{2} \mathrm{CO}_{3}(63.6 \mathrm{mg}, 0.6 \mathrm{mmol}, 3.0$ equiv.) were dissolved in a mixed solvent of $1,2,4,5-\mathrm{C}_{6} \mathrm{H}_{2} \mathrm{~F}_{4}$ and DMA $(2.5 \mathrm{~mL}, v / v=4: 1)$ under $\mathrm{N}_{2}$, and the mixture was stirred for $30 \mathrm{~min}$. Then, alkylarenes 1 ( $0.2 \mathrm{mmol}, 1.0$ equiv.), NFSI-2 (230 $\mathrm{mg}, 0.6 \mathrm{mmol}, 3.0$ equiv.) and alkynylating reagents 2 (0.8 mmol, 4.0 equiv.) were added sequentially, and the tube was sealed with Teflon septum. The reaction was stirred at room temperature for $60 \mathrm{~h}$. The reaction was quenched by a short pad of silica gel with a gradient eluent of petroleum ether and ethyl acetate, solvent was removed under vacuum, and the residue was purified by column chromatography on silica gel with a gradient eluent of petroleum ether and ethyl acetate to give the desired product $\mathbf{3}$ or $\mathbf{4}$. These results were summaried in Table 2.

\section{Late-Stage Functionalization and Synthetic Applications}




\subsection{Late-Stage Functionalization of Splitomicin}

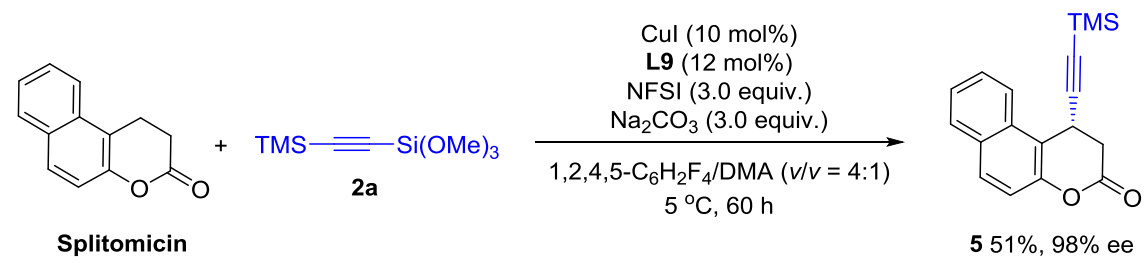

In a dried sealed $10 \mathrm{~mL}$ Schlenk tube, $\mathbf{L 9}$ (9.6 mg, $0.024 \mathrm{mmol}, 12 \mathrm{~mol} \%)$, CuI (3.8 mg, $0.02 \mathrm{mmol}, 10 \mathrm{~mol} \%$ ) and anhydrous $\mathrm{Na}_{2} \mathrm{CO}_{3}$ (63.6 mg, $0.6 \mathrm{mmol}, 3.0$ equiv.) were dissolved in a mixed solvent of $1,2,4,5-\mathrm{C}_{6} \mathrm{H}_{2} \mathrm{~F}_{4}$ and DMA $(2.5 \mathrm{~mL}, v / v=4: 1)$ under $\mathrm{N}_{2}$, and the mixture was stirred for $30 \mathrm{~min}$ and cooled down to $5{ }^{\circ} \mathrm{C}$. Then, Splitomicin $(39.7 \mathrm{mg}, 0.2$ mmol, 1.0 equiv.), NFSI (189.2 $\mathrm{mg}, 0.6 \mathrm{mmol}, 3.0$ equiv.) and $\mathbf{2 a}(174.7 \mathrm{mg}, 0.8 \mathrm{mmol}, 4.0$ equiv.) were added sequentially, and the tube was sealed with Teflon septum. The reaction was stirred at $5{ }^{\circ} \mathrm{C}$ for $60 \mathrm{~h}$. According to the above-mentioned workup procedure by column chromatography $(\mathrm{PE} / \mathrm{EA}=15: 1)$, product $5(29.8 \mathrm{mg}, 51 \%$ yield, $98 \%$ ee $)$ was obtained as a white solid.

${ }^{1} \mathrm{H}$ NMR $\left(400 \mathrm{MHz}, \mathrm{CDCl}_{3}\right) \delta 7.99(\mathrm{~d}, J=8.4 \mathrm{~Hz}, 1 \mathrm{H}), 7.87(\mathrm{~d}, J=8.0 \mathrm{~Hz}, 1 \mathrm{H}), 7.83(\mathrm{~d}, J=$ $9.2 \mathrm{~Hz}, 1 \mathrm{H}), 7.63$ (t, $J=8.0 \mathrm{~Hz}, 1 \mathrm{H}), 7.50$ (t, $J=8.4 \mathrm{~Hz}, 1 \mathrm{H}), 7.26$ (d, $J=8.8 \mathrm{~Hz}, 1 \mathrm{H}), 4.58$ $(\mathrm{dd}, J=6.8,1.6 \mathrm{~Hz}, 1 \mathrm{H}), 3.20(\mathrm{dd}, J=15.6,1.6 \mathrm{~Hz}, 1 \mathrm{H}), 2.92(\mathrm{dd}, J=15.6,6.8 \mathrm{~Hz}, 1 \mathrm{H}), 0.07$ $(\mathrm{s}, 9 \mathrm{H}) ;{ }^{13} \mathrm{C} \mathrm{NMR}\left(100 \mathrm{MHz}, \mathrm{CDCl}_{3}\right) \delta 166.33,148.89,130.93,130.30,129.96,128.71$, $127.58,125.34,122.74,117.69,115.41,102.41,89.16,35.14,24.77,-0.20$; IR (neat): $v=$ 2962, 2161, 1755, 1465, 1352, 1259, 1159, 1081, 1046, 1017, 837, 797, 760, $750 \mathrm{~cm}^{-1}$; HRMS: $\mathrm{m} / \mathrm{z}(\mathrm{EI})$ calculated for $\left(\mathrm{C}_{18} \mathrm{H}_{18} \mathrm{O}_{2} \mathrm{Si}\right) \quad[\mathrm{M}]^{+}:$294.1071, found: 294.1070; melting point: $109-111^{\circ} \mathrm{C}$.

$[\alpha]_{\mathrm{D}}^{30.7}=-131.96\left(c 0.9, \mathrm{CHCl}_{3}\right)$.

HPLC (AD-H, 0.46*25 cm, $5 \mu \mathrm{m}$, hexane/isopropanol = 98/2, flow $0.7 \mathrm{~mL} / \mathrm{min}$, detection at $214 \mathrm{~nm}$ ) retention time $=10.01 \mathrm{~min}$ (major) and $11.68 \mathrm{~min}$ (minor).

\subsection{Synthetic Applications}

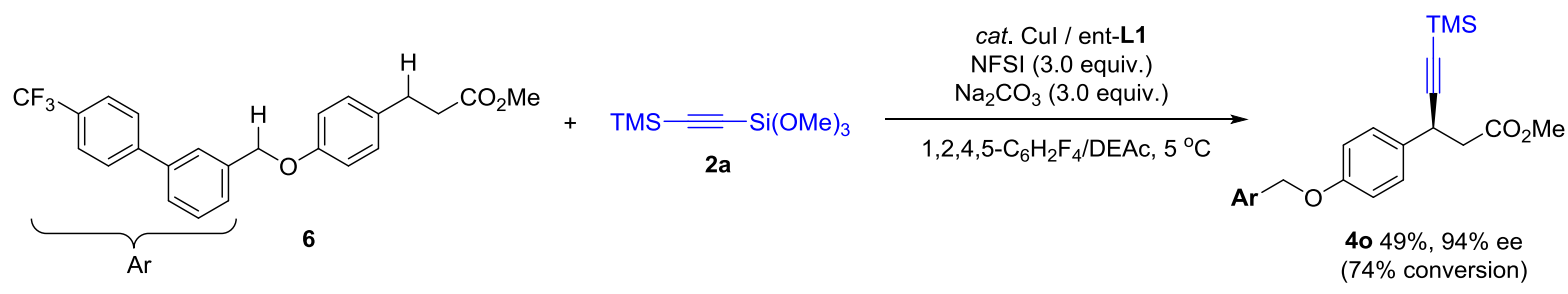

In a dried sealed $10 \mathrm{~mL}$ Schlenk tube, ent-L1 (12.3 mg, $0.024 \mathrm{mmol}, 12 \mathrm{~mol} \%), \mathrm{CuI}$ (3.8 $\mathrm{mg}, 0.02 \mathrm{mmol}, 10 \mathrm{~mol} \%$ ) and anhydrous $\mathrm{Na}_{2} \mathrm{CO}_{3}(63.6 \mathrm{mg}, 0.6 \mathrm{mmol}, 3.0$ equiv.) were dissolved in a mixed solvent of $1,2,4,5-\mathrm{C}_{6} \mathrm{H}_{2} \mathrm{~F}_{4}$ and DEAc ( $N, N$-diethylacetamide) ( $2.5 \mathrm{~mL}$, $v / v=4: 1)$ under $N_{2}$, the mixture was stirred for $30 \mathrm{~min}$ and cooled down to $5{ }^{\circ} \mathrm{C}$. Then, methyl 
3-(4-((4'-(trifluoromethyl)-[1,1'-biphenyl]-3-yl)methoxy)phenyl)propanoate 6 (82.9 mg, 0.2 mmol, 1.0 equiv.), NFSI (189.2 $\mathrm{mg}, 0.6 \mathrm{mmol}, 3.0$ equiv.) and $\mathbf{2 a}(174.7 \mathrm{mg}, 0.8 \mathrm{mmol}, 4.0$ equiv.) were added sequentially, and the tube was sealed with Teflon septum. The reaction was stirred at $5{ }^{\circ} \mathrm{C}$ for $4.5 \mathrm{~d}$. According to the same workup process by column chromatography $(\mathrm{PE} / \mathrm{EA}=8: 1)$, product $40(50.5 \mathrm{mg}, 49 \%$ yield, $94 \%$ ee $)$ was obtained as colorless oil.

${ }^{1} \mathrm{H}$ NMR (400 MHz, $\left.\mathrm{CDCl}_{3}\right) \delta 7.70(\mathrm{~s}, 4 \mathrm{H}), 7.66(\mathrm{~s}, 1 \mathrm{H}), 7.56(\mathrm{~d}, J=6.8 \mathrm{~Hz}, 1 \mathrm{H}), 7.52-7.46$ $(\mathrm{m}, 2 \mathrm{H}), 7.31(\mathrm{~d}, J=8.8 \mathrm{~Hz}, 2 \mathrm{H}), 6.96(\mathrm{~d}, J=8.4 \mathrm{~Hz}, 2 \mathrm{H}), 5.12(\mathrm{~s}, 2 \mathrm{H}), 4.15(\mathrm{t}, J=7.6 \mathrm{~Hz}$, 1H), $3.66(\mathrm{~s}, 3 \mathrm{H}), 2.80(\mathrm{dd}, J=15.2,8.0 \mathrm{~Hz}, 1 \mathrm{H}), 2.69$ (dd, $J=15.2,7.6 \mathrm{~Hz}, 1 \mathrm{H}), 0.17(\mathrm{~s}, 9 \mathrm{H})$; ${ }^{13} \mathrm{C}$ NMR (400 MHz, $\mathrm{CDCl}_{3}$ ) $\delta 171.26,157.80,144.37,140.14,137.83,132.67,129.46$ (q, $J$ $=32.2 \mathrm{~Hz}), 129.26,128.52,127.47,127.23,126.92,126.34,125.72(\mathrm{q}, J=3.9 \mathrm{~Hz}), 124.24(\mathrm{q}$, $J=269.7 \mathrm{~Hz}), 114.91,106.64,87.60,69.84,51.71,43.40,34.33,0.01 ;{ }^{19} \mathrm{~F}$ NMR $(376 \mathrm{MHz}$, $\left.\mathrm{CDCl}_{3}\right) \delta-62.44(\mathrm{~s}, 3 \mathrm{~F})$; IR (neat): $v=2955,2174,1738,1611,1509,1324,1247,1163$, 1122, 1111, 1070, 1016, 839, 789, 759, $699 \mathrm{~cm}^{-1}$; HRMS: m/z (EI) calculated for $\left(\mathrm{C}_{29} \mathrm{H}_{29} \mathrm{~F}_{3} \mathrm{O}_{3} \mathrm{Si}\right)[\mathrm{M}]^{+}:$510.1833, found: 510.1840 .

$[\alpha]_{\mathrm{D}}^{23.1}=16.3\left(c 0.67, \mathrm{CHCl}_{3}\right)$.

HPLC (OD-H, 0.46*25 cm, $5 \mu \mathrm{m}$, hexane/isopropanol = 95/5, flow $0.7 \mathrm{~mL} / \mathrm{min}$, detection at $254 \mathrm{~nm}$ ) retention time $=7.74 \mathrm{~min}$ (minor) and $10.25 \mathrm{~min}$ (major).

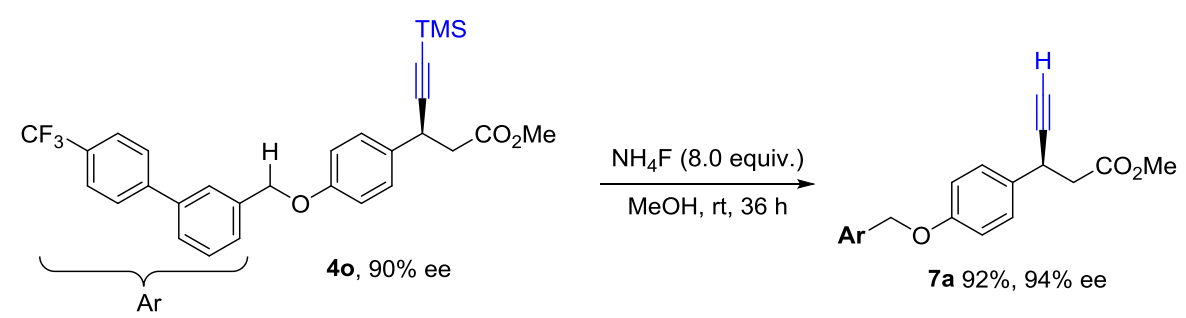

To a solution of $4 \mathbf{0}(51 \mathrm{mg}, 0.1 \mathrm{mmol})$ in $\mathrm{MeOH}(2 \mathrm{~mL}), \mathrm{NH}_{4} \mathrm{~F}(29.6 \mathrm{mg}, 0.8 \mathrm{mmol})$ was added. The mixture was stirred at room temperature for $36 \mathrm{~h}$. After the reaction was completed, the organic solvent was removed under vacuum, and the residue was purifed by column chromatography $(\mathrm{PE} / \mathrm{EA}=8: 1)$ to give the product $7 \mathbf{a}(40.1 \mathrm{mg}, 92 \%$ yield, $94 \%$ ee $)$ as colorless oil.

${ }^{1} \mathrm{H}$ NMR (400 MHz, $\left.\mathrm{CDCl}_{3}\right) \delta 7.69(\mathrm{~s}, 4 \mathrm{H}), 7.65$ (s, 1H), $7.56(\mathrm{~d}, J=7.2 \mathrm{~Hz}, 1 \mathrm{H}), 7.51-7.45$ $(\mathrm{m}, 2 \mathrm{H}), 7.32(\mathrm{~d}, J=8.8 \mathrm{~Hz}, 2 \mathrm{H}), 6.96(\mathrm{~d}, J=8.4 \mathrm{~Hz}, 2 \mathrm{H}), 5.11(\mathrm{~s}, 2 \mathrm{H}), 4.16-4.11(\mathrm{~m}, 1 \mathrm{H})$, $3.67(\mathrm{~s}, 3 \mathrm{H}), 2.83(\mathrm{dd}, J=15.2,8.0 \mathrm{~Hz}, 1 \mathrm{H}), 2.71(\mathrm{dd}, J=15.2,6.8 \mathrm{~Hz}, 1 \mathrm{H}), 2.28(\mathrm{~d}, J=2.4$ $\mathrm{Hz}, 1 \mathrm{H}) ;{ }^{13} \mathrm{C} \mathrm{NMR}\left(100 \mathrm{MHz}, \mathrm{CDCl}_{3}\right) \delta 171.19,157.89,144.36,140.13,137.77,132.29$, $129.45(\mathrm{q}, J=32.1 \mathrm{~Hz}), 129.26,128.44,127.46,127.22,126.92,126.31,125.71(\mathrm{q}, J=3.7$ $\mathrm{Hz}), 124.23$ (q, $J=271.1 \mathrm{~Hz}), 115.00,84.62,71.24,69.84,51.81,42.86,33.04 ;{ }^{19} \mathrm{~F}$ NMR $\left(376 \mathrm{MHz}, \mathrm{CDCl}_{3}\right) \delta-62.42$ (s, 3F); IR (neat): $v=3294,1736,1612,1509,1323,1241,1161$, 1110, 1070, 1016, 842, 830, 789, $643 \mathrm{~cm}^{-1}$; HRMS: m/z (EI) calculated for $\left(\mathrm{C}_{26} \mathrm{H}_{21} \mathrm{~F}_{3} \mathrm{O}_{3}\right)[\mathrm{M}]^{+}$: 438.1437, found: 438.1450 . 
These data are consistent with the reports in the literature. ${ }^{\mathrm{S} 3}$

$[\alpha]_{\mathrm{D}}^{20.0}=3.40\left(c 1.06, \mathrm{CHCl}_{3}\right)$.

HPLC (OD-H, 0.46*25 cm, $5 \mu \mathrm{m}$, hexane/isopropanol $=95 / 5$, flow $0.7 \mathrm{~mL} / \mathrm{min}$, detection at $254 \mathrm{~nm}$ ) retention time $=17.56 \mathrm{~min}$ (minor) and $22.94 \mathrm{~min}$ (major).

\section{Synthetic Transformations}

\subsection{Removing the TMS Group in 3a}

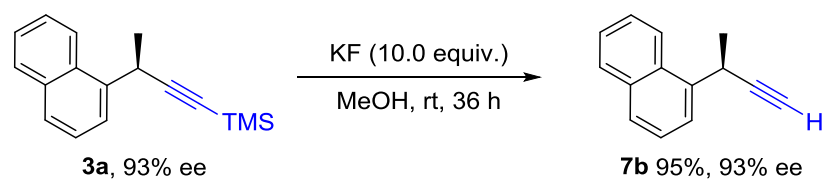

$\mathrm{KF}(116 \mathrm{mg}, 2.0 \mathrm{mmol})$ was added to a solution of $\mathbf{3 a}(50.5 \mathrm{mg}, 0.2 \mathrm{mmol})$ in $\mathrm{MeOH}(2$ $\mathrm{mL}$ ), and the mixture was stirred at room temperature for $36 \mathrm{~h}$. After the reaction was completed, the organic solvent was removed under vacuum, and the residue was purified by column chromatography $(\mathrm{PE} / \mathrm{EA}=100: 1)$ to give product $\mathbf{7 b}(34.2 \mathrm{mg}, 95 \%$ yield, $93 \%$ ee $)$ as colorless oil.

${ }^{1} \mathrm{H}$ NMR $\left(400 \mathrm{MHz}, \mathrm{CDCl}_{3}\right) \delta 8.09(\mathrm{~d}, J=8.0 \mathrm{~Hz}, 1 \mathrm{H}), 7.88-7.86(\mathrm{~m}, 1 \mathrm{H}), 7.76(\mathrm{~d}, J=7.6 \mathrm{~Hz}$, 2H), 7.56-7.45 (m, 3H), 4.56-4.50 (qd, $J=7.2,2.4 \mathrm{~Hz}, 1 \mathrm{H}), 2.31(\mathrm{~d}, J=2.4 \mathrm{~Hz}, 1 \mathrm{H}), 1.66$ (d, $J=7.2 \mathrm{~Hz}, 3 \mathrm{H}) ;{ }^{13} \mathrm{C}$ NMR $\left(100 \mathrm{MHz}, \mathrm{CDCl}_{3}\right) \delta 138.13,133.95,130.43,129.00,127.58$, $126.06,125.62,125.53,124.10,122.98,87.23,70.39,28.13,23.10$; IR (neat): $v=3293,3048$, 2977, 2931, 2113, 1596, 1510, 1449, 1395, 797, 774, 632, 517, $433 \mathrm{~cm}^{-1}$; HRMS: m/z (EI) calculated for $\left(\mathrm{C}_{14} \mathrm{H}_{12}\right)[\mathrm{M}]^{+}:$: 180.0934 , found: 180.0932 .

$[\alpha]_{\mathrm{D}}^{30.8}=18.40\left(c 0.71, \mathrm{CHCl}_{3}\right)$.

HPLC (AD-H, 0.46*25 cm, $5 \mu \mathrm{m}$, hexane/isopropanol = 100/0, flow $0.7 \mathrm{~mL} / \mathrm{min}$, detection at $214 \mathrm{~nm}$ ) retention time $=12.10 \mathrm{~min}$ (minor) and $13.02 \mathrm{~min}$ (major).

\subsection{Click Reaction}

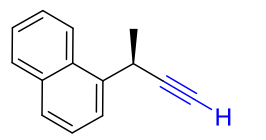

7b, $93 \%$ ee

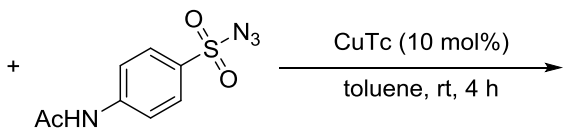

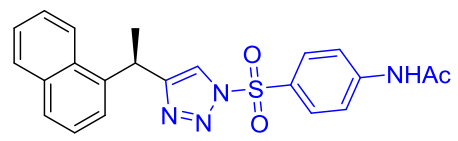

$882 \%, 93 \%$ ee

To a solution of CuTc $(3.8 \mathrm{mg}, 0.02 \mathrm{mmol})$ and $7 \mathbf{b}(36 \mathrm{mg}, 0.2 \mathrm{mmol})$ in toluene $(2 \mathrm{~mL})$, 4-acetamidobenzenesulfonyl azide $(39.5 \mathrm{mg}, 0.2 \mathrm{mmol}$ ) was added, and the mixture was stirred at room temperature for $4 \mathrm{~h}$. After the reaction was completed, the organic solvent was removed under vacuum, and the residue was purified by column chromatography (PE/EA = $3: 1)$ to give the product $8(69.2 \mathrm{mg}, 82 \%$ yield, $93 \%$ ee) as a white solid. 
${ }^{1} \mathrm{H}$ NMR $\left(400 \mathrm{MHz}\right.$, DMSO-d $\left.d_{6}\right) \delta 10.58(\mathrm{~s}, 1 \mathrm{H}), 8.74(\mathrm{~s}, 1 \mathrm{H}), 8.21-8.19(\mathrm{~m}, 1 \mathrm{H}), 8.08-8.05$ $(\mathrm{m}, 2 \mathrm{H}), 7.93-7.86(\mathrm{~m}, 3 \mathrm{H}), 7.79(\mathrm{~d}, J=8.4 \mathrm{~Hz}, 1 \mathrm{H}), 7.55-7.48(\mathrm{~m}, 2 \mathrm{H}), 7.43(\mathrm{t}, J=7.6 \mathrm{~Hz}$, $1 \mathrm{H}), 7.28(\mathrm{~d}, J=7.6 \mathrm{~Hz}, 1 \mathrm{H}), 5.13(\mathrm{q}, J=7.2 \mathrm{~Hz}, 1 \mathrm{H}), 2.10(\mathrm{~s}, 3 \mathrm{H}), 1.70(\mathrm{~d}, J=6.8 \mathrm{~Hz}, 3 \mathrm{H})$; ${ }^{13} \mathrm{C}$ NMR (100 MHz, DMSO- $\left.d_{6}\right) \delta 169.61,152.16,146.17,140.05,133.59,130.62,130.02$, 128.84, 127.97, 127.16, 126.31, 125.72, 125.70, 124.30, 123.29, 122.20, 119.24, 31.61, 24.34, 20.99; IR (neat): $v=3326,3119,1699,1589,1527,1392,1319,1193,1174,1018,1004,776$, 728, 647, 631, 579, $541 \mathrm{~cm}^{-1}$; HRMS: m/z (ESI) calculated for $\left(\mathrm{C}_{22} \mathrm{H}_{21} \mathrm{~N}_{4} \mathrm{O}_{3} \mathrm{~S}\right)[\mathrm{M}+\mathrm{H}]^{+}$: 421.1329, found: 421.1337 . The melting point (8) couldn't be determined, for it went bad at high temperature.

$[\alpha]_{\mathrm{D}}^{30.1}=-85.15(c 0.69, \mathrm{MeOH})$.

HPLC (AD-H, 0.46*25 cm, 5 $\mu \mathrm{m}$, hexane/isopropanol = 85/15, flow $0.7 \mathrm{~mL} / \mathrm{min}$, detection at $214 \mathrm{~nm}$ ) retention time $=27.52 \mathrm{~min}$ (major) and $38.21 \mathrm{~min}$ (minor).

\subsection{Reduction of the C-C Triple Bond by DIBAL-H}

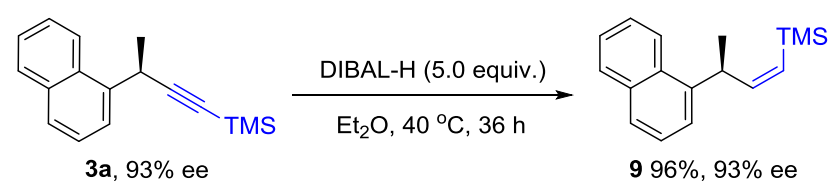

To a solution of $3 \mathrm{a}(50.5 \mathrm{mg}, 0.2 \mathrm{mmol})$ in $\mathrm{Et}_{2} \mathrm{O}(2 \mathrm{~mL})$, DIBAL-H $(1.0 \mathrm{~mL}, 1.0 \mathrm{M}$ solution in hexanes) was added, then the mixture was stirred at $40{ }^{\circ} \mathrm{C}$ for $36 \mathrm{~h}$. After the reaction was completed, aqueous solution of $\mathrm{NaOH}$ was added to quench extra DIABL-H, and the solution was extracted by $\mathrm{Et}_{2} \mathrm{O}$. The combined organic layer was dried over $\mathrm{MgSO}_{4}$, and filtered. After the removal of the solvent, the residue was purified by column chromatography (PE) to give product 9 (48.7 $\mathrm{mg}, 96 \%$ yield, $93 \%$ ee) as colorless oil.

${ }^{1} \mathrm{H}$ NMR $\left(400 \mathrm{MHz}, \mathrm{CDCl}_{3}\right) \delta 8.16(\mathrm{~d}, J=8.4 \mathrm{~Hz}, 1 \mathrm{H}), 7.89-7.87(\mathrm{~m}, 1 \mathrm{H}), 7.76-7.72(\mathrm{~m}, 1 \mathrm{H})$, 7.57-7.44 (m, 4H), $6.68(\mathrm{dd}, J=14.0,9.6 \mathrm{~Hz}, 1 \mathrm{H}), 5.64(\mathrm{~d}, J=14.0 \mathrm{~Hz}, 1 \mathrm{H}), 4.50-4.43$ (qd, $J$ $=9.6,6.4 \mathrm{~Hz}, 1 \mathrm{H}), 1.51(\mathrm{~d}, J=6.8 \mathrm{~Hz}, 3 \mathrm{H}), 0.18(\mathrm{~s}, 9 \mathrm{H}) ;{ }^{13} \mathrm{C} \mathrm{NMR}\left(100 \mathrm{MHz}, \mathrm{CDCl}_{3}\right) \delta$ $152.70,142.29$, 133.95, 131.24, 128.92, 128.56, 126.62, 125.65, 125.61, 125.31, 123.44, 122.93, 38.21, 23.09, 0.25; IR (neat): $v=2955,1597,1247,832,794,773,689.671 \mathrm{~cm}^{-1}$; HRMS: $\mathrm{m} / \mathrm{z}$ (EI) calculated for $\left(\mathrm{C}_{17} \mathrm{H}_{22} \mathrm{Si}\right)[\mathrm{M}]^{+}: 254.1485$, found: 254.1491 .

$[\alpha]_{\mathrm{D}}^{30.8}=39.11\left(c 0.93, \mathrm{CHCl}_{3}\right)$.

HPLC (OD-H, 0.46*25 cm, 5 $\mu \mathrm{m}$, hexane/isopropanol $=100 / 0$, flow $0.7 \mathrm{~mL} / \mathrm{min}$, detection at $214 \mathrm{~nm}$ ) retention time $=19.31 \mathrm{~min}$ (major) and $21.57 \mathrm{~min}$ (minor).

\subsection{Pd/C-Catalyzed Hydrogenation of the C-C Triple Bond}

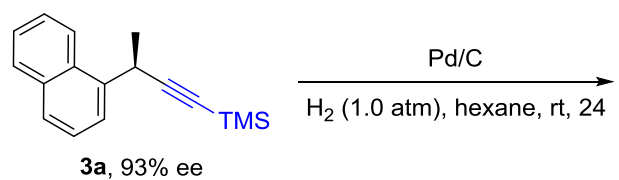

S11 
To a solution of 3a $(50.5 \mathrm{mg}, 0.2 \mathrm{mmol})$ in hexane $(2.0 \mathrm{~mL}), 10 \mathrm{wt} \% \mathrm{Pd} / \mathrm{C}(5.1 \mathrm{mg})$ was added under $\mathrm{H}_{2}(1.0 \mathrm{~atm})$, and the hydrogenation was performed at room temperature for $24 \mathrm{~h}$. After filtration through a short pad of celite, the organic solvent was removed under vacuum. The residue was purified by column chromatography (PE) to give product $\mathbf{1 0}(50.5 \mathrm{mg}, 98 \%$ yield, $86 \%$ ee) as colorless oil.

${ }^{1} \mathrm{H}$ NMR $\left(400 \mathrm{MHz}, \mathrm{CDCl}_{3}\right) \delta 8.16(\mathrm{~d}, J=8.0 \mathrm{~Hz}, 1 \mathrm{H}), 7.90-7.88(\mathrm{~m}, 1 \mathrm{H}), 7.73(\mathrm{~d}, J=8.0 \mathrm{~Hz}$, $1 \mathrm{H}), 7.56-7.47(\mathrm{~m}, 3 \mathrm{H}), 7.40(\mathrm{~d}, J=7.2 \mathrm{~Hz}, 1 \mathrm{H}), 3.60-3.52(\mathrm{~m}, 1 \mathrm{H}), 1.91-1.82(\mathrm{~m}, 1 \mathrm{H}), 1.73-$ $1.64(\mathrm{~m}, 1 \mathrm{H}), 1.41(\mathrm{~d}, J=7.2 \mathrm{~Hz}, 3 \mathrm{H}), 0.67-0.59(\mathrm{~m}, 1 \mathrm{H}), 0.55-0.47(\mathrm{~m}, 1 \mathrm{H}),-0.01(\mathrm{~s}, 9 \mathrm{H})$; ${ }^{13} \mathrm{C}$ NMR $\left(100 \mathrm{MHz}, \mathrm{CDCl}_{3}\right) \delta 143.85,133.94,131.86,128.91,126.12,125.60,125.58$, $125.17,123.25,122.41,36.78,31.99,21.28,14.75,-1.79$; IR (neat): $v=3047,2953,2917$, 1596, 1509, 1454, 1246, 1024, 859, 830, 795, 775, 690, $437 \mathrm{~cm}^{-1}$; HRMS: m/z (EI) calculated for $\left(\mathrm{C}_{17} \mathrm{H}_{24} \mathrm{Si}\right)[\mathrm{M}]^{+}:$256.1642, found: 256.1648 .

$[\alpha]_{\mathrm{D}}^{30.9}=-43.96\left(c 1.0, \mathrm{CHCl}_{3}\right)$.

HPLC (OD-H, 0.46*25 cm, 5 $\mu \mathrm{m}$, hexane/isopropanol $=100 / 0$, flow $0.7 \mathrm{~mL} / \mathrm{min}$, detection at $214 \mathrm{~nm}$ ) retention time $=24.25 \mathrm{~min}$ (major) and $33.73 \mathrm{~min}$ (minor).

\subsection{TBD-Catalyzed Isomerization}
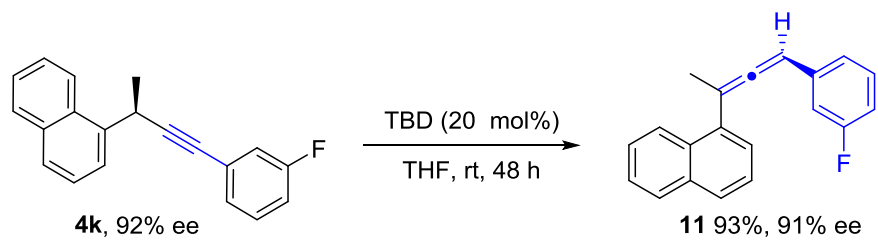

To a solution of $\mathbf{4 k}(55 \mathrm{mg}, 0.2 \mathrm{mmol})$ in THF (4 mL), triazabicyclodecene (TBD, $5.6 \mathrm{mg}$, $0.04 \mathrm{mmol}$ ) was added, and the mixture was stirred at room temperature for $48 \mathrm{~h}$. After the reaction was completed, the organic solvent was removed under vacuum, and the residue was purified by column chromatography (PE/EA = 100:1) to give product 11 (51.2 mg, 93\% yield, $91 \%$ ee) as colorless oil.

${ }^{1} \mathrm{H}$ NMR $\left(400 \mathrm{MHz}, \mathrm{CDCl}_{3}\right) \delta 8.27(\mathrm{~d}, J=7.6 \mathrm{~Hz}, 1 \mathrm{H}), 7.90-7.88(\mathrm{~m}, 1 \mathrm{H}), 7.82(\mathrm{~d}, J=7.6 \mathrm{~Hz}$, $1 \mathrm{H}), 7.56-7.49(\mathrm{~m}, 4 \mathrm{H}), 7.32-7.26(\mathrm{~m}, 1 \mathrm{H}), 7.17-7.11(\mathrm{~m}, 2 \mathrm{H}), 6.95-6.90(\mathrm{~m}, 1 \mathrm{H}), 6.30$ (q, $J=$ $2.8 \mathrm{~Hz}, 1 \mathrm{H}), 2.38(\mathrm{~d}, J=2.8 \mathrm{~Hz}, 3 \mathrm{H}) ;{ }^{13} \mathrm{C} \mathrm{NMR}\left(100 \mathrm{MHz}, \mathrm{CDCl}_{3}\right) \delta 205.50,163.15(\mathrm{~d}, J=$ $243.5 \mathrm{~Hz}), 137.47(\mathrm{~d}, J=7.7 \mathrm{~Hz}), 135.91,133.94,130.83,129.96(\mathrm{~d}, J=8.0 \mathrm{~Hz}), 128.51$, 127.84, 126.09, 125.81, 125.46, 125.30, 125.14, 122.67 (d, $J=2.6 \mathrm{~Hz}), 113.66$ (d, $J=20.6$ $\mathrm{Hz}), 113.41(\mathrm{~d}, J=21.8 \mathrm{~Hz}), 103.66,93.29(\mathrm{~d}, J=2.0 \mathrm{~Hz}), 21.13 ;{ }^{19} \mathrm{~F}$ NMR $(376 \mathrm{MHz}$, $\left.\mathrm{CDCl}_{3}\right) \delta$-113.53-113.60 (m, 1F); IR (neat): $v=2980,1610,1584,1487,1445,1247,1131$, 872, 798, 772, 741, $681 \mathrm{~cm}^{-1}$; HRMS: $\mathrm{m} / \mathrm{z}$ (EI) calculated for $\left(\mathrm{C}_{20} \mathrm{H}_{15} \mathrm{~F}\right)[\mathrm{M}]^{+}:$274.1152, found: 274.1155 .

$[\alpha]_{\mathrm{D}}^{31.0}=-478.84\left(c 0.94, \mathrm{CHCl}_{3}\right)$. 
HPLC (AD-H, 0.46*25 cm, $5 \mu \mathrm{m}$, hexane/isopropanol = 99/1, flow $0.7 \mathrm{~mL} / \mathrm{min}$, detection at $214 \mathrm{~nm}$ ) retention time $=6.28 \mathrm{~min}$ (minor) and $7.04 \mathrm{~min}$ (major).

\subsection{Oxidation of the C-C Triple Bond}

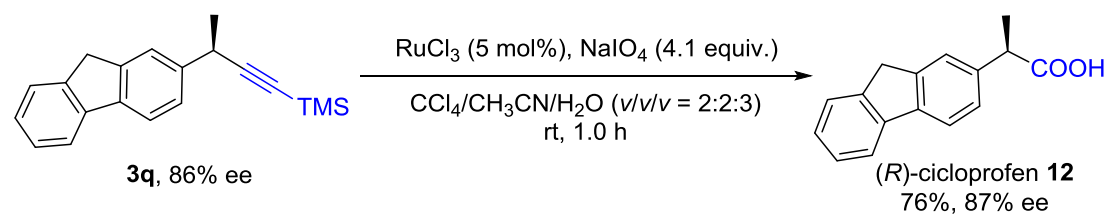

To a solution of $\mathbf{3 q}(23.8 \mathrm{mg}, 0.082 \mathrm{mmol})$ in a mixed solvent of $\mathrm{CCl}_{4}, \mathrm{CH}_{3} \mathrm{CN}$ and $\mathrm{H}_{2} \mathrm{O}$ $(0.7 \mathrm{~mL}, v / v / v 2: 2: 3), \mathrm{RuCl}_{3}(0.8 \mathrm{mg}, 5 \mathrm{~mol} \%)$ and sodium periodate (72 mg, 4.1 equiv.) were added, then the mixture was stirred vigorously at room temperature for $1 \mathrm{~h}$. After reaction completion, the mixture was extracted with dichloromethane $(3 \times 5 \mathrm{~mL})$. The combined organic phase was dried over $\mathrm{Na}_{2} \mathrm{SO}_{4}$. The organic solvent was removed under vacuum, and the residue was purified via a preparative plate $(\mathrm{PE} / \mathrm{EA}=3: 2)$ to give $(R)$-cicloprofen 12 (14.8 $\mathrm{mg}, 76 \%, 86 \%$ ee) as a white solid.

${ }^{1} \mathrm{H}$ NMR $\left(400 \mathrm{MHz}, \mathrm{CDCl}_{3}\right) \delta$ 7.76-7.72 (m, 2H), $7.52(\mathrm{~d}, J=10.4 \mathrm{~Hz}, 2 \mathrm{H}), 7.38-7.29(\mathrm{~m}$, $3 \mathrm{H}), 3.88(\mathrm{~s}, 2 \mathrm{H}), 3.82(\mathrm{q}, J=7.2 \mathrm{~Hz}, 1 \mathrm{H}), 1.56(\mathrm{~d}, J=7.2 \mathrm{~Hz}, 3 \mathrm{H}) ;{ }^{13} \mathrm{C}$ NMR $(100 \mathrm{MHz}$, $\left.\mathrm{CDCl}_{3}\right) \delta 179.95,143.76,143.29,141.27,141.09,138.33,126.73,126.70,126.31,124.99$, 124.19, 119.99, 119.86, 45.32, 36.84, 18.33; IR (neat): $v=2962,1692,1410,1259,1014$, 794, 766, 733, 611, $460 \mathrm{~cm}^{-1}$; HRMS: m/z (EI) calculated for $\left(\mathrm{C}_{16} \mathrm{H}_{14} \mathrm{O}_{2}\right)[\mathrm{M}]^{+}: 238.0988$, found: 238.0995 .

These data are consitent with those reported in the literature. ${ }^{\mathrm{S}}$

$[\alpha]_{\mathrm{D}}^{30.9}=-26.76(c 0.33, \mathrm{MeOH})$.

HPLC (AD-H, 0.46*25 cm, $5 \mu \mathrm{m}$, hexane/isopropanol/TFA = 90/10/0.1, flow $0.7 \mathrm{~mL} / \mathrm{min}$, detection at $254 \mathrm{~nm}$ ) retention time $=12.76 \mathrm{~min}$ (major) and $13.80 \mathrm{~min}$ (minor).

\section{Mechanistic Studies}

\subsection{Synthesis of Alkynylsilanes}

Alkynylsilanes were synthesized according to the reported literature. ${ }^{\text {55a }}$

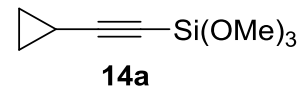

(Cyclopropylethynyl)trimethoxysilane (14a): ${ }^{1} \mathrm{H}$ NMR $\left(400 \mathrm{MHz}, \mathrm{CDCl}_{3}\right) \delta 3.58(\mathrm{~s}, 9 \mathrm{H})$, 1.36-1.29 (m, 1H), 0.86-0.81 (m, 4H); ${ }^{13} \mathrm{C}$ NMR (100 MHz, $\left.\mathrm{CDCl}_{3}\right) \delta 111.05,69.35,50.71$, 8.89, 0.08; IR (neat): $v=2944,2843,2166,1456,1352,1191,1072,983,712,566,486 \mathrm{~cm}^{-1}$; HRMS: m/z (EI) calculated for $\left(\mathrm{C}_{8} \mathrm{H}_{14} \mathrm{O}_{3} \mathrm{Si}\right)[\mathrm{M}]^{+}$: 186.0712, found: 186.0709. 


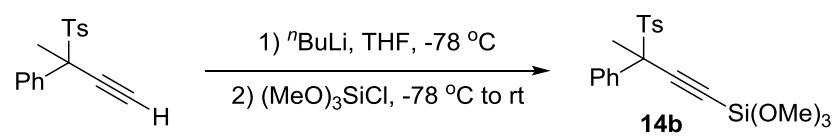

1-Methyl-4-((2-phenylbut-3-yn-2-yl)sulfonyl)benzene was synthesized according to the literature. ${ }^{\text {S5b }}$ To a solution of 1-methyl-4-((2-phenylbut-3-yn-2-yl)sulfonyl)benzene (1.422 g, $5 \mathrm{mmol})$ in dried THF $(10 \mathrm{~mL})$ was slowly added ${ }^{n} \mathrm{BuLi}(2.4 \mathrm{~mL}, 2.5 \mathrm{M}$ in solution in hexanes) at $-78{ }^{\circ} \mathrm{C}$ under $\mathrm{N}_{2}$, the reaction mixture was warmed up to room temperature and stirred at room tempeprature for $30 \mathrm{~min}$. Then, $(\mathrm{MeO})_{3} \mathrm{SiCl}(10 \mathrm{mmol}, 2.0$ equiv.) was dropwise added to the mixture at $-78{ }^{\circ} \mathrm{C}$, and the reaction was warmed up to room temperature and stirred at room tempeprature overnight. After reaction completion, THF was removed under vacuum, dried $\mathrm{Et}_{2} \mathrm{O}(50 \mathrm{~mL})$ was then poured into the residue, the resulting mixture was sonicated for $30 \mathrm{~min}$. After filtration, $\mathrm{Et}_{2} \mathrm{O}$ was removed and $1.97 \mathrm{~g}$ the residue was used directly for the enantioselective benzylic C-H bond alkynylation. IR (neat): $v=2944,2844$, 2182 (C-C triple bond), 1596, 1492, 1451, 1315, 1188, 1144, 1065, 946, 815, 763, 708, 683, 670, 573, 527, 450; HRMS: m/z (EI) calculated for $\left(\mathrm{C}_{20} \mathrm{H}_{24} \mathrm{O}_{5} \mathrm{SSi}\right)[\mathrm{M}]^{+}:$404.1114, found: 404.1110. Note: The product $\mathbf{1 4 b}$ can not be isolated by flash column chromatography or distillation under high vacuum. THF and $\mathrm{Et}_{2} \mathrm{O}$ shoud be very dry, all the apparatuses shoud be dried before they were used.

\subsection{Using $\mathrm{CBrCl}_{3}$ as a Radical Scavenger}

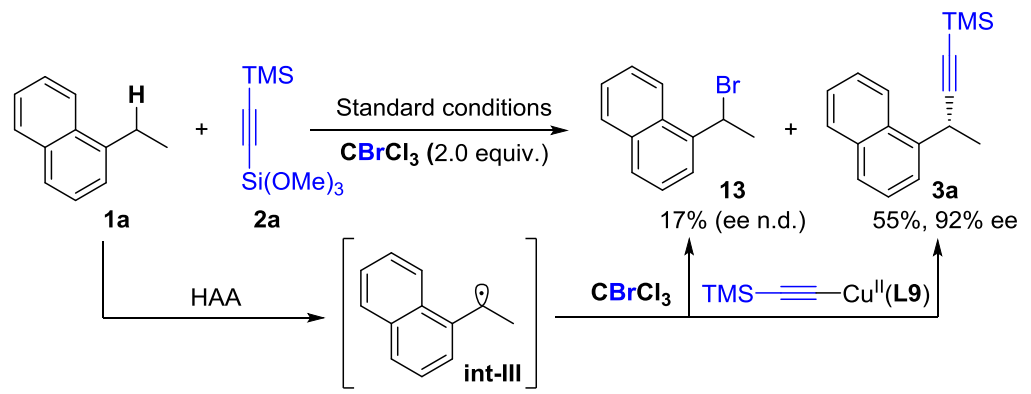

In a dried sealed $10 \mathrm{~mL}$ Schlenk tube, $\mathbf{L 9}(9.6 \mathrm{mg}, 0.024 \mathrm{mmol}, 12 \mathrm{~mol} \%)$, CuI (3.8 mg, $0.02 \mathrm{mmol}, 10 \mathrm{~mol} \%)$ and anhydrous $\mathrm{Na}_{2} \mathrm{CO}_{3}(63.6 \mathrm{mg}, 0.6 \mathrm{mmol})$ were dissolved in 1,2,4,5$\mathrm{C}_{6} \mathrm{H}_{2} \mathrm{~F}_{4}$ and DMA $(2.5 \mathrm{~mL}, v / v=4: 1)$ under $\mathrm{N}_{2}$, and the mixture was stirred for $30 \mathrm{~min}$. Then, $1 \mathbf{a}$ (31.2 mg, $0.2 \mathrm{mmol}, 1.0$ equiv.), NFSI-2 (230 mg, $0.6 \mathrm{mmol}, 3.0$ equiv.), $2 \mathbf{a}$ (174.7 mg, $0.8 \mathrm{mmol}, 4.0$ equiv.) and $\mathrm{CBrCl}_{3}(79.3 \mathrm{mg}, 0.4 \mathrm{mmol}, 2.0$ equiv.) were added. The tube was sealed with Teflon septum, the reaction mixture was stirred at room temperature for $39 \mathrm{~h}$. The reaction was quenched by a short pad of silica gel $(\mathrm{PE} / \mathrm{EA}=10: 1)$, the solvent was removed under vacuum, and the residue was analyzed by ${ }^{1} \mathrm{H}$ NMR using $\mathrm{CH}_{2} \mathrm{Br}_{2}$ as an internal standard. The formation of $\mathbf{1 3}$ indicated the involvement of a benzylic radical.

\subsection{Competition KIE Experiment}




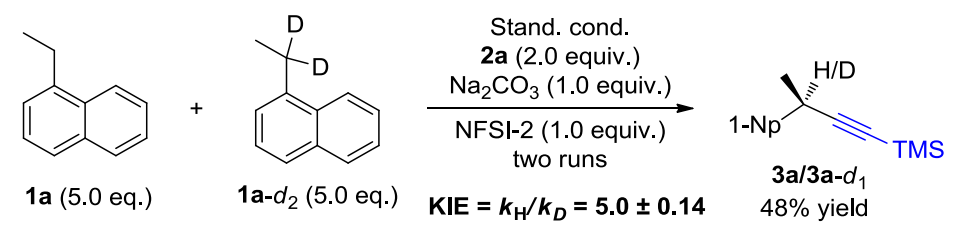

The reaction was performed using NFSI-2 as a limiting reagent. In a dried sealed $10 \mathrm{~mL}$ Schlenk tube, $\mathbf{L 9}$ (4.8 mg, $0.012 \mathrm{mmol}, 12 \mathrm{~mol} \%$ ), CuI (1.9 mg, $0.01 \mathrm{mmol}, 10 \mathrm{~mol} \%$ ) and anhydrous $\mathrm{Na}_{2} \mathrm{CO}_{3}(10.6 \mathrm{mg}, 0.1 \mathrm{mmol})$ were dissolved in $1,2,4,5-\mathrm{C}_{6} \mathrm{H}_{2} \mathrm{~F}_{4}$ and DMA (1.25 $\mathrm{mL}, v / v=4: 1)$ under $\mathrm{N}_{2}$, and the mixture was stirred for $30 \mathrm{~min}$. Then, $1 \mathrm{a}(78.1 \mathrm{mg}, 0.5 \mathrm{mmol}$, 5.0 equiv.), 1 a- $d_{2}$ (79.1 mg, $0.5 \mathrm{mmol}, 5.0$ equiv.), NFSI-2 (38.3 mg, $0.1 \mathrm{mmol}, 1.0$ equiv.) and $2 \mathbf{a}(43.7 \mathrm{mg}, 0.2 \mathrm{mmol}, 2.0$ equiv.) were added. The tube was sealed with Teflon septum, the reaction mixture was stirred at room temperature for $38 \mathrm{~h}$. The reaction was quenched by a short pad of silica gel $(\mathrm{PE} / \mathrm{EA}=10: 1)$, the solvent was removed under vacuum, and the residue was purified by column chromatography (PE) to give the mixture products $\mathbf{3 a} / \mathbf{3 a}-d_{1}$ as colorless oil. The KIE ratio was determined by ${ }^{1} \mathrm{H}$ qNMR spectroscopy.

First run: $\mathrm{KIE}=4.86$ (48\% isolated yield); second run: $\mathrm{KIE}=5.14$ (49\% isolated yield).

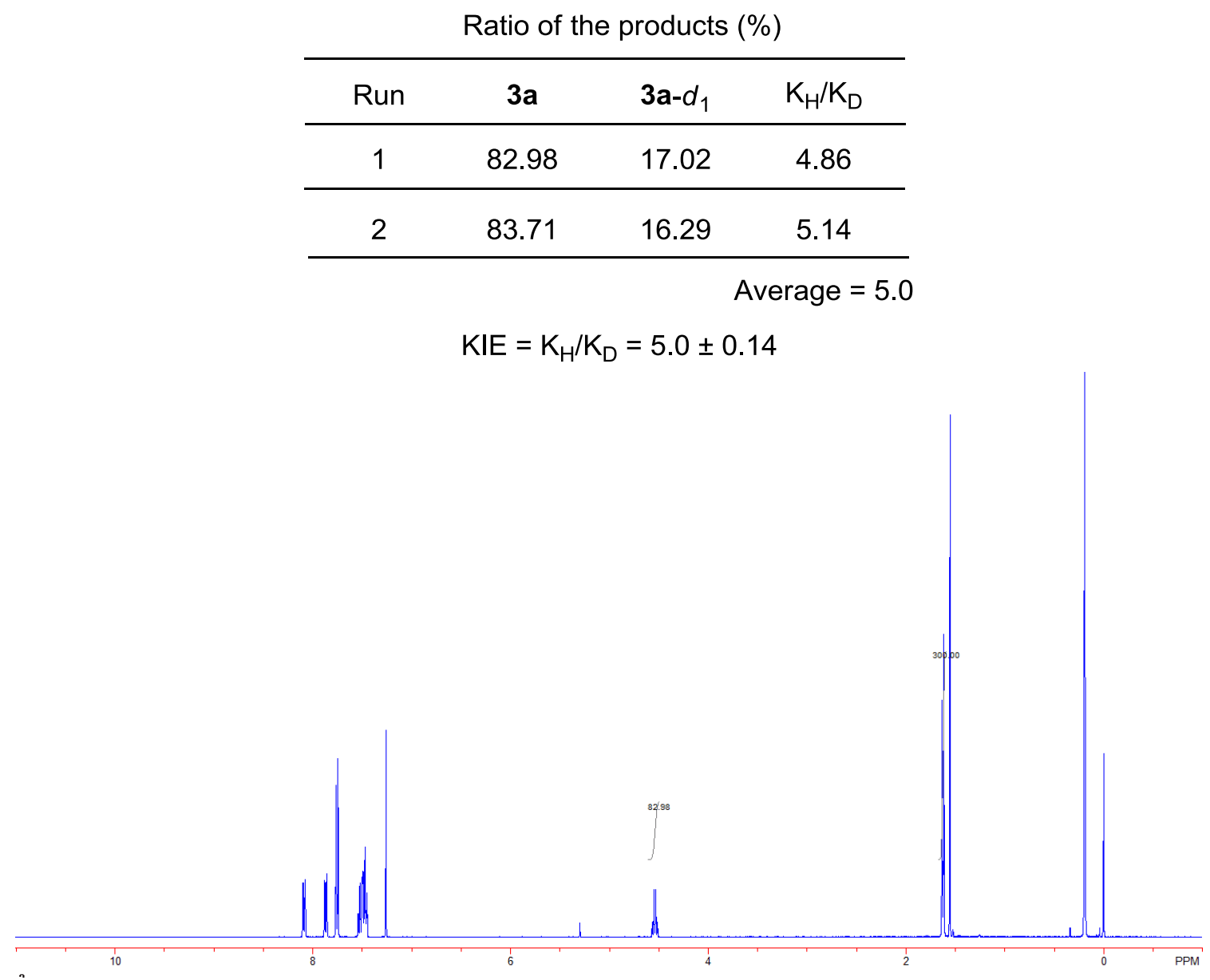




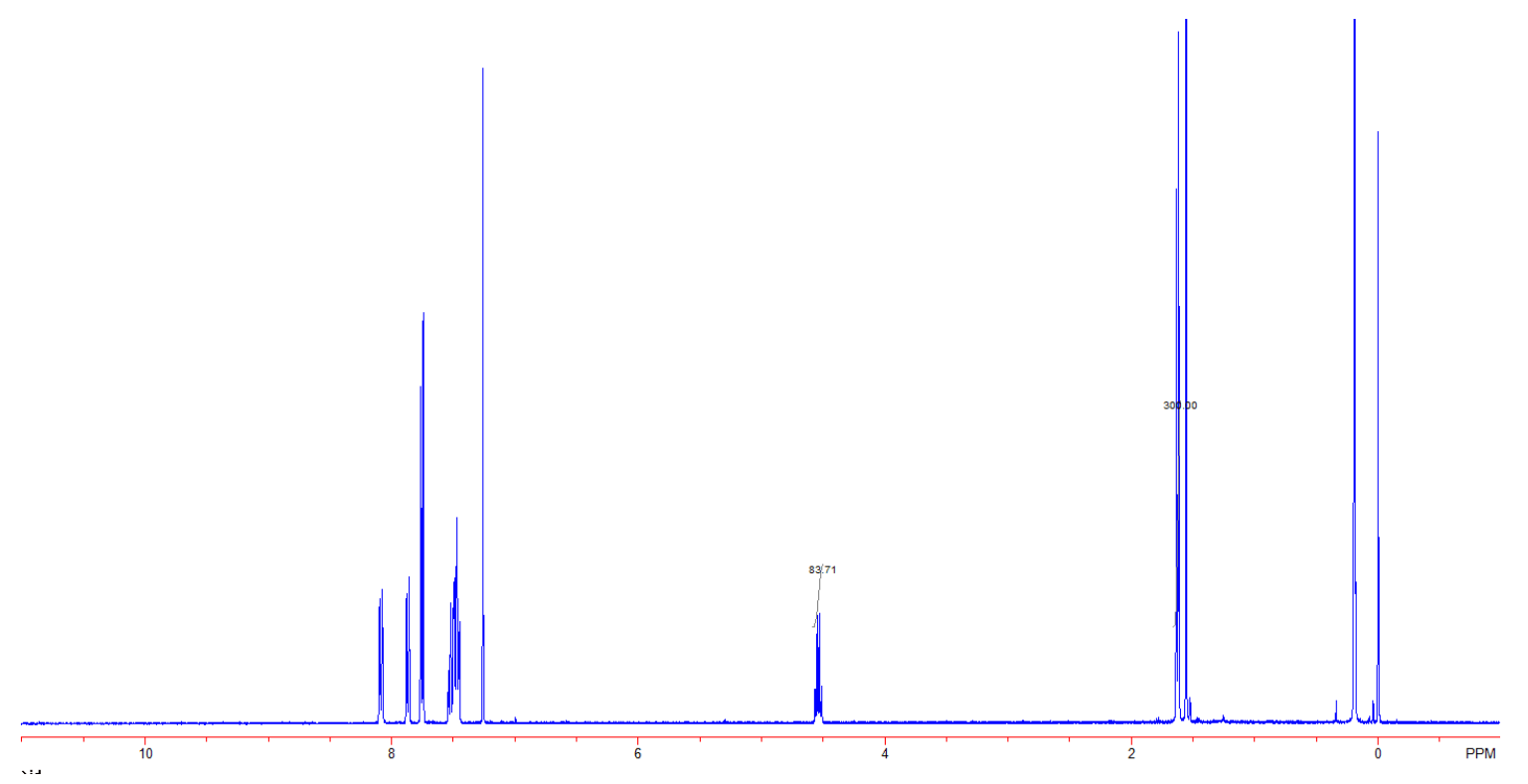

\subsection{Independent-Rate KIE Experiments}

For the enantioselective benzylic $\mathrm{C}-\mathrm{H}$ bond alkynylation is heterogeneous, the reaction of 1a and the reaction of $\mathbf{1 a}-d_{2}$ under the standard conditions were carried out at various time independently. In a glove box, a homogenous solution of $\mathrm{CuI}(38.1 \mathrm{mg}, 0.2 \mathrm{mmol})$ and $\mathbf{L 9}$ (95.6 mg, $0.24 \mathrm{mmol}$ ) in dried and degassed DCM was prepared in a $2.0 \mathrm{~mL}$ volumetric flask. In the glove box, anhydrous $\mathrm{Na}_{2} \mathrm{CO}_{3}(31.8 \mathrm{mg}, 3.0$ equiv.) and $100 \mu \mathrm{L}$ the solution of $\mathrm{CuI} / \mathrm{L9}$ in DCM were added to a dried sealed $10 \mathrm{~mL}$ Schlenk tube, and DCM was removed under high vacuum very carefully. Then, the residue was dissolved in 1,2,4,5- $\mathrm{C}_{6} \mathrm{H}_{2} \mathrm{~F}_{4} / \mathrm{DMA}(1.25$ $\mathrm{mL}, v / v=4: 1)$ under $\mathrm{N}_{2}, \mathbf{1 a} / \mathbf{1 a}-d_{2}(0.1 \mathrm{mmol}, 1.0$ equiv.), NFSI-2 (115 mg, $0.3 \mathrm{mmol}, 3.0$ equiv.) and 2a (87.4 mg, $0.4 \mathrm{mmol}, 4.0$ equiv.) were added. The tube was sealed with Teflon septum, the reaction mixture was stirred at room temperature for the indicated time. All the reactions were quenched through a short pad of silica gel $(\mathrm{PE} / \mathrm{EA}=10: 1)$ and solvent was removed under vacuum. The residue was analyzed by GC using dodecane as an internal standard. These studies revealed an independent KIE of 2.27, suggesting that $\mathrm{C}-\mathrm{H}$ bond cleavage is only partially rate-determining.

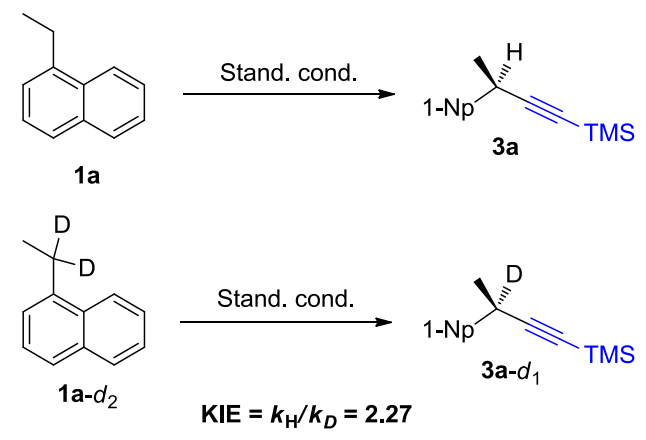



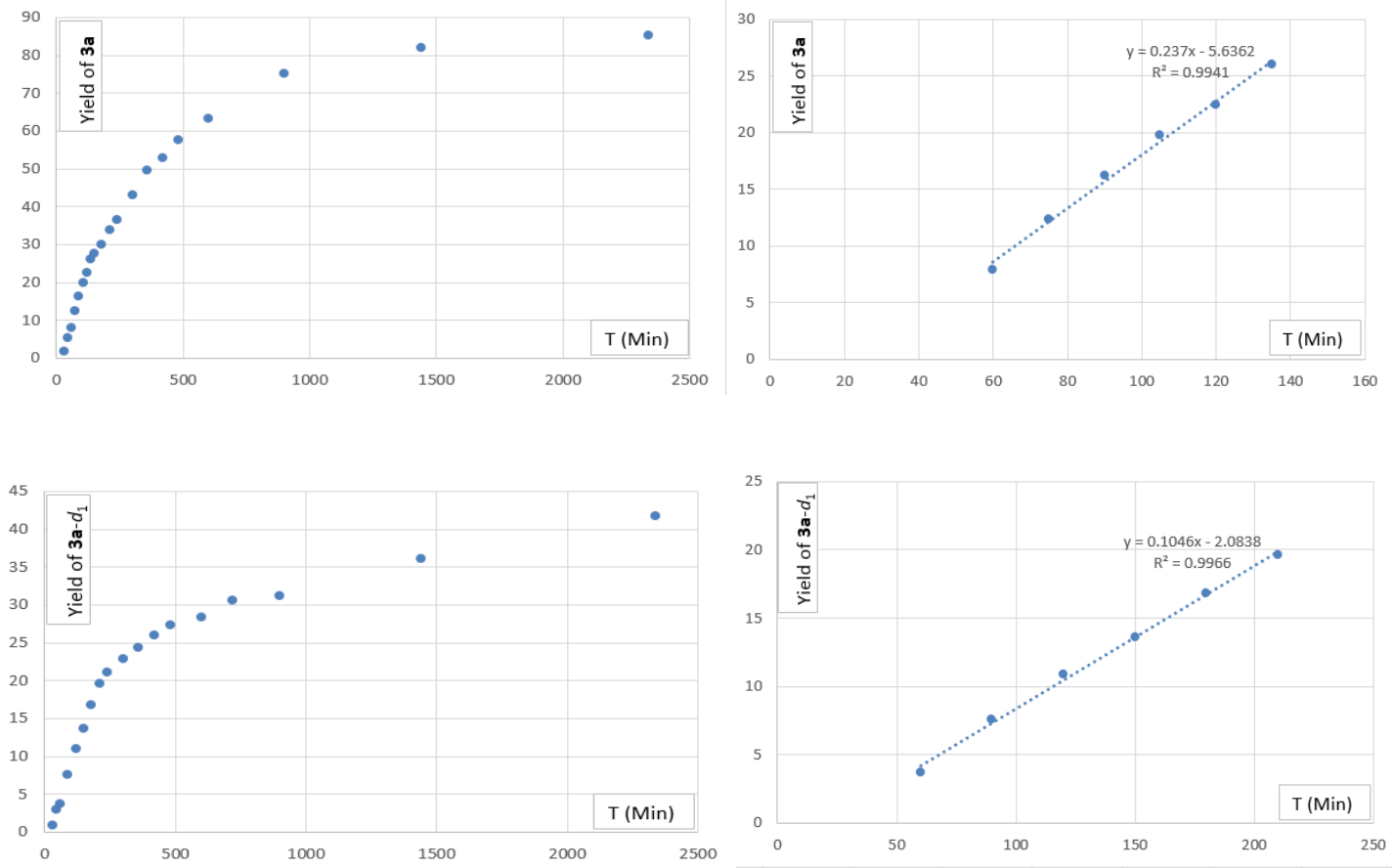

\subsection{Experimental Mechanistic Studies}

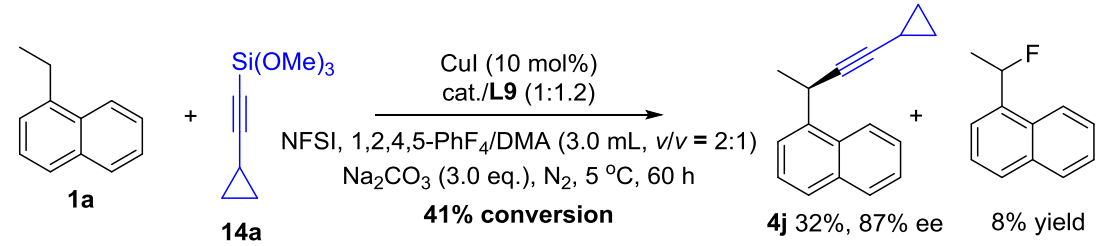

In a dried sealed $10 \mathrm{~mL}$ Schlenk tube, $\mathbf{L 9}$ (9.6 mg, $0.024 \mathrm{mmol}, 12 \mathrm{~mol} \%)$, CuI (3.8 mg, $0.02 \mathrm{mmol}, 10 \mathrm{~mol} \%$ ) and anhydrous $\mathrm{Na}_{2} \mathrm{CO}_{3}(63.6 \mathrm{mg}, 0.6 \mathrm{mmol}, 3.0$ equiv.) were dissolved in a mixed solvent of $1,2,4,5-\mathrm{C}_{6} \mathrm{H}_{2} \mathrm{~F}_{4}$ and DMA $(3.0 \mathrm{~mL}, v / v=2: 1)$ under $\mathrm{N}_{2}$, and the mixture was stirred for $30 \mathrm{~min}$. Then, $1 \mathrm{a}(31.2 \mathrm{mg}, 0.2 \mathrm{mmol}, 1.0$ equiv.), NFSI (189.2 mg, 0.6 mmol, 3.0 equiv.) and (cyclopropylethynyl)trimethoxysilane $\mathbf{1 4 a}$ (149 mg, $0.8 \mathrm{mmol}, 4.0$ equiv.) were added sequentially, the tube was sealed with Teflon septum, and the reaction was stirred at $5{ }^{\circ} \mathrm{C}$ for $60 \mathrm{~h}$. The reaction was quenched by a short pad of silica gel (PE/EA = 4:1), the solvent was removed under vacuum and the residue was purified by column chromatography (PE) to give product $\mathbf{4 j}$ (14.2 $\mathrm{mg}$, 32\% yield, $87 \%$ ee) as colorless oil. $41 \%$ Conversion (1a) and $8 \%$ yield of the $\mathrm{C}-\mathrm{H}$ fluorination product were determined by ${ }^{1} \mathrm{H}$ NMR using $\mathrm{CH}_{2} \mathrm{Br}_{2}$ as an internal standard. Excellent mass balance was observed for the reaction.

$[\alpha]_{\mathrm{D}}^{25.5}=14.99\left(c 0.41, \mathrm{CHCl}_{3}\right)$.

HPLC (OD-H, 0.46*25 cm, $5 \mu \mathrm{m}$, hexane/isopropanol $=99 / 1$, flow $0.7 \mathrm{~mL} / \mathrm{min}$, detection at $214 \mathrm{~nm}$ ) retention time $=8.93 \mathrm{~min}$ (major) and $13.88 \mathrm{~min}$ (minor). 


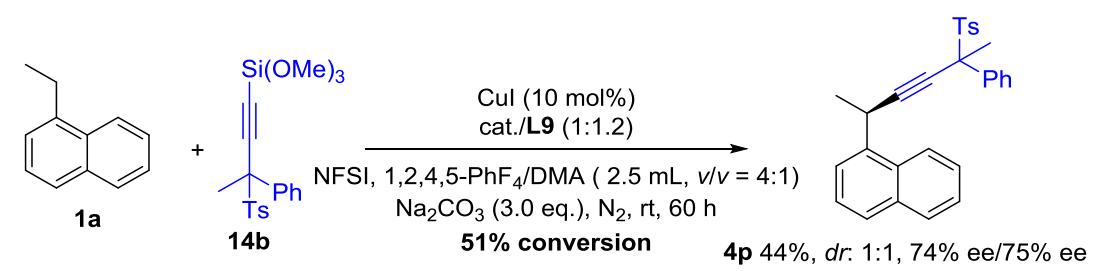

In a dried sealed $10 \mathrm{~mL}$ Schlenk tube, $\mathbf{L 9}$ (9.6 mg, $0.024 \mathrm{mmol}, 12 \mathrm{~mol} \%)$, CuI (3.8 mg, $0.02 \mathrm{mmol}, 10 \mathrm{~mol} \%)$ and anhydrous $\mathrm{Na}_{2} \mathrm{CO}_{3}(63.6 \mathrm{mg}, 0.6 \mathrm{mmol}, 3.0$ equiv.) were dissolved in a mixed solvent of $1,2,4,5-\mathrm{C}_{6} \mathrm{H}_{2} \mathrm{~F}_{4}$ and DMA $(2.5 \mathrm{~mL}, v / v=4: 1)$ under $\mathrm{N}_{2}$, and the mixture was stirred for $30 \mathrm{~min}$. Then, $1 \mathbf{a}(31.2 \mathrm{mg}, 0.2 \mathrm{mmol}, 1.0$ equiv.), NFSI (189.2 mg, $0.6 \mathrm{mmol}, 3.0$ equiv.) and the unisolable alkynylsilane $\mathbf{1 4 b}(0.8 \mathrm{mmol}, 4.0$ equiv. $)$ were added sequentially, the tube was sealed with Teflon septum, and the reaction was stirred at room temperature for $60 \mathrm{~h}$. The reaction was quenched by a short pad of silica gel $(\mathrm{PE} / \mathrm{EA}=4: 1)$, the solvent was removed under vacuum. The residue was purified by RP-HPLC $\left(\mathrm{CH}_{3} \mathrm{CN} / \mathrm{H}_{2} \mathrm{O}\right.$ = 9:1) to give product $\mathbf{4 p}(25.5 \mathrm{mg}, 29 \%$ yield, dr: $1.1: 1,74 \%$ ee/75\% ee) as very sticky colorless oil. 51\% Conversion (1a) and 44\% ${ }^{1} \mathrm{H}$ NMR yield (4p) were determined by using $\mathrm{CH}_{2} \mathrm{Br}_{2}$ as an internal standard. Excellent mass balance was also observed for the reaction.

${ }^{1} \mathrm{H} \mathrm{NMR}\left(400 \mathrm{MHz}, \mathrm{CDCl}_{3}\right) \delta 8.10-8.06(\mathrm{~m}, 1 \mathrm{H}), 7.91-7.87(\mathrm{~m}, 1 \mathrm{H}), 7.80-7.78(\mathrm{~d}, J=8.4 \mathrm{~Hz}$, $1 \mathrm{H}), 7.66(\mathrm{t}, J=6.8 \mathrm{~Hz}, 1 \mathrm{H}), 7.55-7.44(\mathrm{~m}, 5 \mathrm{H}), 7.34-7.24(\mathrm{~m}, 5 \mathrm{H}), 6.91(\mathrm{t}, J=6.8 \mathrm{~Hz}, 2 \mathrm{H})$ $4.62(\mathrm{q}, J=7.2 \mathrm{~Hz}, 1 \mathrm{H}), 2.31-2.30$ (diastereomers, 3H), 2.13 (s, 3H), 1.71-1.69 (diastereomers, $3 \mathrm{H}) ;{ }^{13} \mathrm{C} \mathrm{NMR}\left(100 \mathrm{MHz}, \mathrm{CDCl}_{3}\right) \delta 144.44,144.41,137.98,137.93,134.79$, $133.97,131.69,131.66,130.82,130.80,130.49,130.48,129.07,128.97,128.78,128.45$, $128.42,127.84,127.63,126.12,126.07,125.63,125.60,124.32,124.29,123.12,92.77,92.74$, $79.67,79.62,67.24,28.70,28.63,22.68,22.61,22.36,22.30,21.55,21.53$; IR (neat): $v=$ 3054, 2980, 2932, 2248, 1593, 1498, 1448, 1386, 1142, 1061, 909, 783, 731, 690, 642, 576, $517 \mathrm{~cm}^{-1}$; HRMS: m/z (ESI) calculated for $\left(\mathrm{C}_{29} \mathrm{H}_{26} \mathrm{O}_{2} \mathrm{NaS}\right)[\mathrm{M}+\mathrm{Na}]^{+}: 461.1546$, found: 461.1549 .

$[\alpha]_{\mathrm{D}}^{25.5}=-11.71\left(c 0.64, \mathrm{CHCl}_{3}\right)$.

HPLC (OD-H, 0.46*25 cm, $5 \mu \mathrm{m}$, hexane/isopropanol = 97/3, flow $0.7 \mathrm{~mL} / \mathrm{min}$, detection at $214 \mathrm{~nm}$ ) retention time $=23.41 \mathrm{~min}$ (minor) and $33.84 \mathrm{~min}$ (major), $25.94 \mathrm{~min}$ (major) and $29.58 \mathrm{~min}$ (minor).

\subsection{Equimolar Complex of $\mathrm{CuI}$ and $\mathrm{L} 9$ in the Presence of Anhydrous $\mathrm{Na}_{2} \mathrm{CO}_{3}$}

In a glove box, $\mathrm{CuI}(7.6 \mathrm{mg}, 0.04 \mathrm{mmol}), \mathbf{L 9}(15.9 \mathrm{mg}, 0.04 \mathrm{mmol})$ and anhydrous $\mathrm{Na}_{2} \mathrm{CO}_{3}(127.2 \mathrm{mg}, 1.2 \mathrm{mmol})$ were dissolved in $2 \mathrm{~mL} \mathrm{CDCl}_{3}$, and the mixture was stirred at room temperature for $2 \mathrm{~h}$. After filtration in the glove box, the filtrate was directly measured by ${ }^{1} \mathrm{H}$ NMR. The ${ }^{1} \mathrm{H}$ NMR results clearly demonstrated that $\mathbf{L 9}$ couldn't generate a covalent complex with $\mathrm{CuI}$ in the presence of anhydrous $\mathrm{Na}_{2} \mathrm{CO}_{3}$, for $\mathrm{Na}_{2} \mathrm{CO}_{3}$ is probably not basic enough for the deprotonation process. 


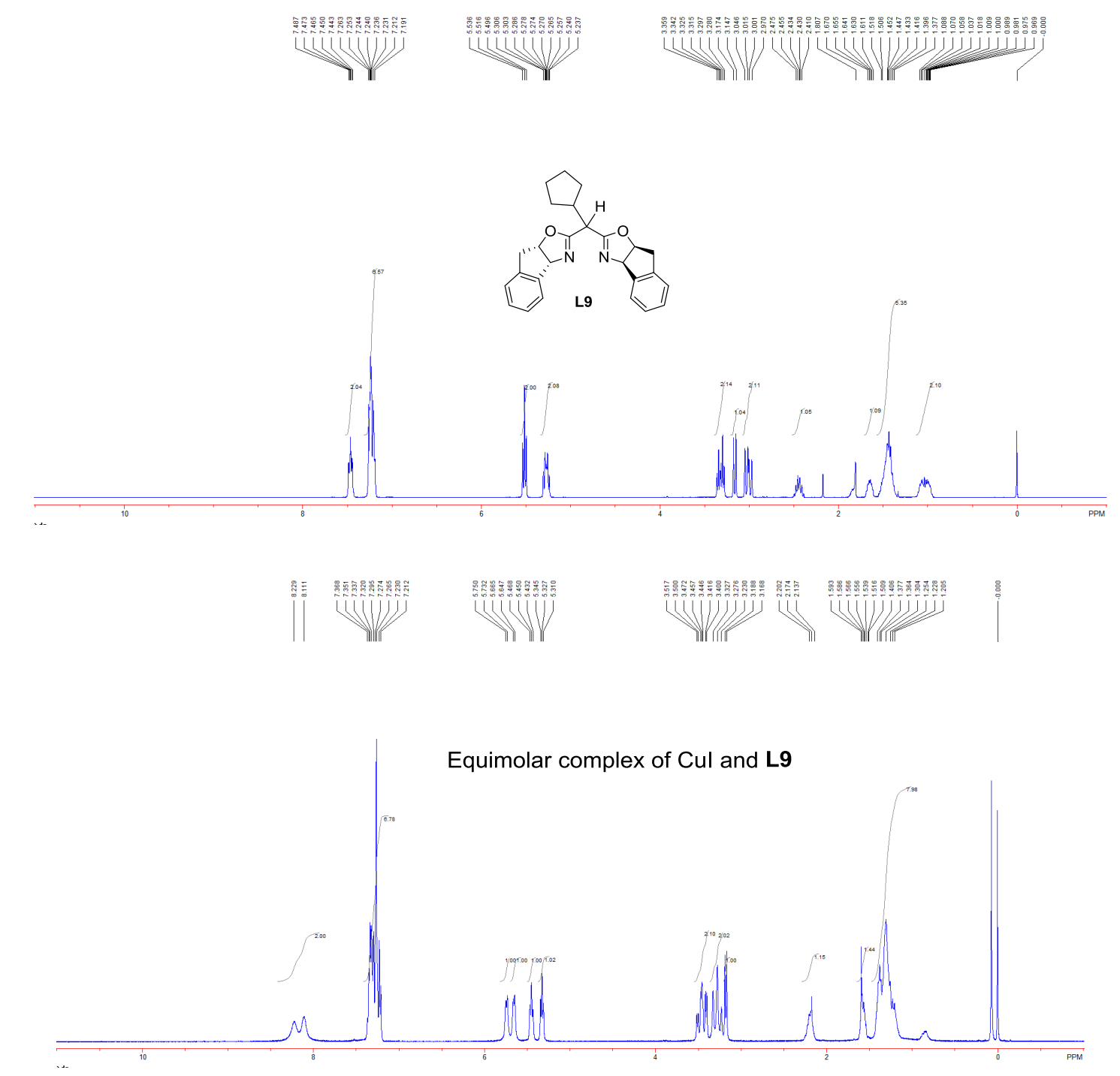

\section{Large-Scale Reactions}

\subsection{Using 1,2,4,5- $\mathrm{C}_{6} \mathrm{H}_{2} \mathrm{~F}_{4}$ and DMA as Solvents}

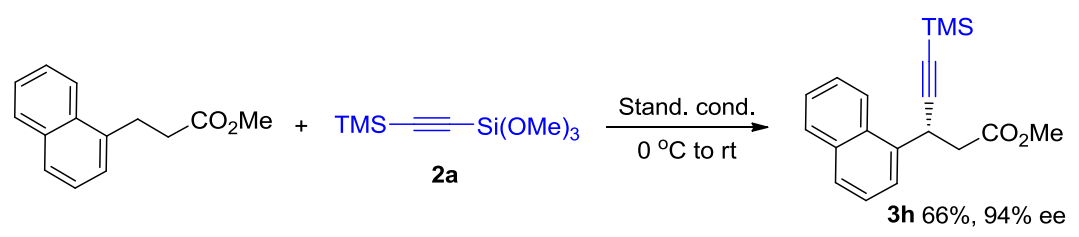

In a dried sealed $250 \mathrm{~mL}$ Schlenk tube, L9 (239.1 mg, $0.6 \mathrm{mmol}, 12 \mathrm{~mol} \%), \mathrm{CuI}(95.2 \mathrm{mg}$, $0.5 \mathrm{mmol}, 10 \mathrm{~mol} \%)$ and anhydrous $\mathrm{Na}_{2} \mathrm{CO}_{3}(1.59 \mathrm{~g}, 15 \mathrm{mmol})$ were dissolved in a mixed solvent of $1,2,4,5-\mathrm{C}_{6} \mathrm{H}_{2} \mathrm{~F}_{4}$ and DMA $(62.5 \mathrm{~mL}, v / v=4: 1)$ under $\mathrm{N}_{2}$, and the mixture was stirred for $30 \mathrm{~min}$ and cooled down to $0{ }^{\circ} \mathrm{C}$. Then, methyl 3-(naphthalen-1-yl)propanoate $\mathbf{1 h}$ (1.07 g, 5.0 mmol, 1.0 equiv.), NFSI-2 (5.75 g, 15.0 mmol, 3.0 equiv.) and 2a (4.37 g, 20 mmol, 4.0 equiv.) were added sequentially, and the tube was sealed with Teflon septum. The reaction mixture was warmed up to room temperature and stirred for $60 \mathrm{~h}$. According to the same workup procedure, the residue was purified by column chromatography (PE/EA = 20:1) 
to give product $3 \mathrm{~h}$ (1.03 g, 66\% yield, $94 \%$ ee) as colorless oil. $75 \%$ Conversion (alkylarene) was determined by using $\mathrm{CH}_{2} \mathrm{Br}_{2}$ as an internal standard.

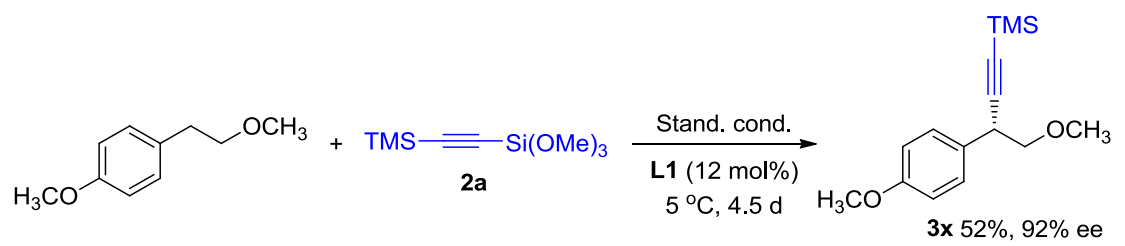

In a dried sealed $250 \mathrm{~mL}$ Schlenk tube, L1 (612.8 mg, $1.2 \mathrm{mmol}, 12 \mathrm{~mol} \%)$, CuI (190.5 mg, $1.0 \mathrm{mmol}, 10 \mathrm{~mol} \%)$ and anhydrous $\mathrm{Na}_{2} \mathrm{CO}_{3}(3.18 \mathrm{~g}, 30 \mathrm{mmol})$ were dissolved in a mixed solvent of $1,2,4,5-\mathrm{C}_{6} \mathrm{H}_{2} \mathrm{~F}_{4}$ and DMA $(125 \mathrm{~mL}, v / v=4: 1)$ under $\mathrm{N}_{2}$, and the mixture was stirred for $30 \mathrm{~min}$ and cooled down to $5{ }^{\circ} \mathrm{C}$. Then, 1-methoxy-4-(2-methoxyethyl)benzene $\mathbf{1 x}$ (1.66 g, 10.0 mmol, 1.0 equiv.), NFSI (9.46 g, 30.0 mmol, 3.0 equiv.) and $2 \mathbf{a}$ (8.74 g, 40 mmol, 4.0 equiv.) were added sequentially, and the tube was sealed with Teflon septum. The reaction mixture was stirred at $5{ }^{\circ} \mathrm{C}$ for $4.5 \mathrm{~d}$. According to the same workup procedure, the residue was purified by column chromatography $(\mathrm{PE} / \mathrm{EA}=10: 1)$ to give product $\mathbf{3 x}(1.36 \mathrm{~g}$, $52 \%$ yield, $92 \%$ ee) as colorless oil. 57\% Conversion (alkylarene) was determined by using $\mathrm{CH}_{2} \mathrm{Br}_{2}$ as an internal standard.

\subsection{Using PhCl and DMA as Solvents}

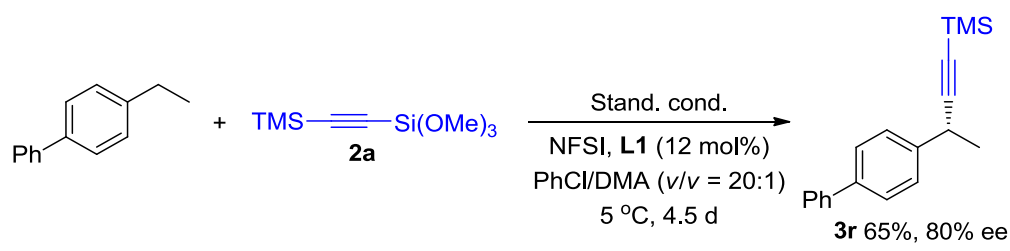

In a dried sealed $250 \mathrm{~mL}$ Schlenk tube, L1 (612.8 mg, $1.2 \mathrm{mmol}, 12 \mathrm{~mol} \%), \mathrm{CuI}(190.5 \mathrm{mg}$, $1.0 \mathrm{mmol}, 10 \mathrm{~mol} \%)$ and anhydrous $\mathrm{Na}_{2} \mathrm{CO}_{3}(3.18 \mathrm{~g}, 30 \mathrm{mmol})$ were dissolved in a mixed solvent of $\mathrm{PhCl}$ and DMA $(105 \mathrm{~mL}, v / v=20: 1)$ under $N_{2}$, and the mixture was stirred at room temperature for $30 \mathrm{~min}$ and cooled down to $5{ }^{\circ} \mathrm{C}$. Then, 4-ethyl-1,1'-biphenyl (1.83 g, 10 mmol, 1.0 equiv.), NFSI (9.46 g, 30 mmol, 3.0 equiv.) and $\mathbf{2 a}(8.74$ g, 40 mmol, 4.0 equiv.) were added sequentially, and the tube was sealed with Teflon septum. The reaction mixture was stirred at $5{ }^{\circ} \mathrm{C}$ for $4.5 \mathrm{~d}$. According to the same workup procedure, the residue was purified by column chromatography $(\mathrm{PE} / \mathrm{EA}=50: 1)$ to give product $3 \mathrm{r}$ (1.82 $\mathrm{g}, 65 \%$ yield, $80 \%$ ee) as colorless oil. $80 \%$ Conversion (alkylarene) was determined by using $\mathrm{CH}_{2} \mathrm{Br}_{2}$ as an internal standard.

$[\alpha]_{\mathrm{D}}^{24.6}=-0.19\left(c 1.1, \mathrm{CHCl}_{3}\right)$.

HPLC (OD-H, 0.46*25 cm, $5 \mu \mathrm{m}$, hexane/isopropanol $=100 / 0$, flow $0.7 \mathrm{~mL} / \mathrm{min}$, detection at $254 \mathrm{~nm}$ ) retention time $=20.49 \mathrm{~min}$ (major) and $26.82 \mathrm{~min}$ (minor). 


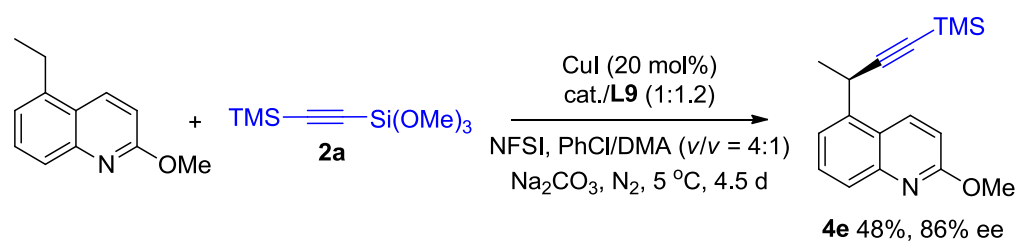

In a dried sealed $250 \mathrm{~mL}$ Schlenk tube, $\mathbf{L 9}$ (669.5 mg, $1.68 \mathrm{mmol}, 24 \mathrm{~mol} \%)$, CuI (266.6 $\mathrm{mg}, 1.4 \mathrm{mmol}, 20 \mathrm{~mol} \%)$ and anhydrous $\mathrm{Na}_{2} \mathrm{CO}_{3}(4.45 \mathrm{~g}, 42 \mathrm{mmol})$ were dissolved in a mixed solvent of $\mathrm{PhCl}$ and DMA $(87.5 \mathrm{~mL}, v / v=4: 1)$ under $N_{2}$, and the mixture was stirred at room temperature for $30 \mathrm{~min}$ and cooled down to $5{ }^{\circ} \mathrm{C}$. Then, 5-ethyl-2-methoxyquinoline (1.31 g, 7 mmol, 1.0 equiv.), NFSI (13.24 g, $42 \mathrm{mmol}, 6.0$ equiv.) and 2a (12.23 g, 56 mmol, 8.0 equiv.) were added sequentially, and the tube was sealed with Teflon septum. The reaction mixture was stirred at $5{ }^{\circ} \mathrm{C}$ for $4.5 \mathrm{~d}$. According to the same workup procedure, the residue was purified by column chromatography (PE/EA $=60: 1)$ to give product $4 \mathbf{e}(950.2 \mathrm{mg}, 48 \%$ yield, $86 \%$ ee) as colorless oil. $50 \%$ Conversion (alkylarene) was determined by using $\mathrm{CH}_{2} \mathrm{Br}_{2}$ as an internal standard.

$[\alpha]_{\mathrm{D}}^{23.5}=-6.32\left(c 1.04, \mathrm{CHCl}_{3}\right)$.

HPLC (OD-H, 0.46*25 cm, $5 \mu \mathrm{m}$, hexane/isopropanol = 99/1, flow $0.7 \mathrm{~mL} / \mathrm{min}$, detection at $214 \mathrm{~nm}$ ) retention time $=5.27 \mathrm{~min}$ (minor) and $5.50 \mathrm{~min}$ (major).

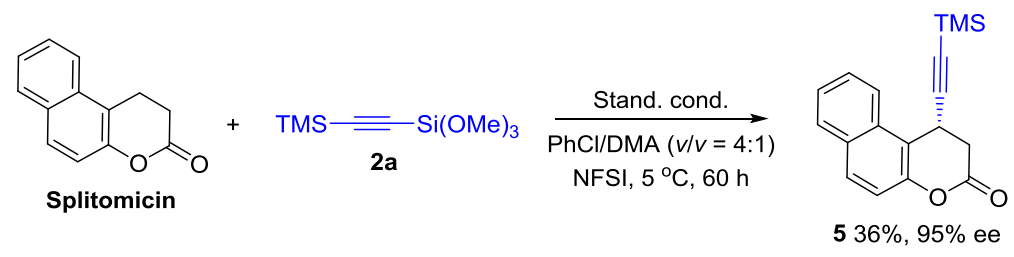

In a dried sealed $250 \mathrm{~mL}$ Schlenk tube, L9 (334.7 mg, $0.84 \mathrm{mmol}, 12 \mathrm{~mol} \%), \mathrm{CuI}$ (133.3 $\mathrm{mg}, 0.7 \mathrm{mmol}, 10 \mathrm{~mol} \%)$ and anhydrous $\mathrm{Na}_{2} \mathrm{CO}_{3}(2.23 \mathrm{~g}, 21 \mathrm{mmol})$ were dissolved in a mixed solvent of $\mathrm{PhCl}$ and DMA $(87.5 \mathrm{~mL}, v / v=4: 1)$ under $\mathrm{N}_{2}$, and the mixture was stirred at room temperature for $30 \mathrm{~min}$ and cooled down to $5{ }^{\circ} \mathrm{C}$. Then, Splitomicin $(1.39 \mathrm{~g}, 7 \mathrm{mmol}$, 1.0 equiv.), NFSI (6.62 g, $21 \mathrm{mmol}, 3.0$ equiv.) and 2a (6.12 g, $28 \mathrm{mmol}, 4.0$ equiv.) were added sequentially, and the tube was sealed with Teflon septum. The reaction mixture was stirred at $5{ }^{\circ} \mathrm{C}$ for $60 \mathrm{~h}$. According to the same workup procedure, the residue was purified by column chromatography $(\mathrm{PE} / \mathrm{EA}=15: 1)$ to give product $5(743.7 \mathrm{mg}, 36 \%$ yield, $95 \%$ ee $)$ as a white solid.

$[\alpha]_{\mathrm{D}}^{26.1}=-127.24\left(c 0.79, \mathrm{CHCl}_{3}\right)$.

HPLC (AD-H, 0.46*25 cm, $5 \mu \mathrm{m}$, hexane/isopropanol = 98/2, flow $0.7 \mathrm{~mL} / \mathrm{min}$, detection at $214 \mathrm{~nm}$ ) retention time $=9.55 \mathrm{~min}$ (major) and $11.12 \mathrm{~min}$ (minor) .

\section{Product Characterization}




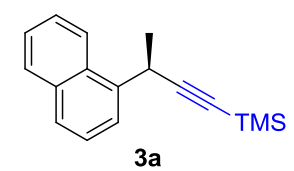

The reaction was conducted on a $0.2 \mathrm{mmol}$ scale according to the general conditions. Column chromatography (PE) yielded product 3a (45.0 mg, 89\% yield, 93\% ee) as colorless oil.

${ }^{1} \mathrm{H}$ NMR (400 MHz, $\left.\mathrm{CDCl}_{3}\right) \delta 8.09(\mathrm{~d}, J=8.4 \mathrm{~Hz}, 1 \mathrm{H}), 7.87(\mathrm{~d}, J=8.0 \mathrm{~Hz}, 1 \mathrm{H}), 7.76(\mathrm{~d}, J=$ $7.6 \mathrm{~Hz}, 2 \mathrm{H}), 7.54-7.46(\mathrm{~m}, 3 \mathrm{H}), 4.55(\mathrm{q}, J=6.8 \mathrm{~Hz}, 1 \mathrm{H}), 1.63(\mathrm{~d}, J=6.8 \mathrm{~Hz}, 3 \mathrm{H}), 0.20$ (s, $9 \mathrm{H}) ;{ }^{13} \mathrm{C} \mathrm{NMR}\left(100 \mathrm{MHz}, \mathrm{CDCl}_{3}\right) \delta 138.64,133.94,130.45,128.93,127.41,125.90,125.65$, 125.44, 124.31, 123.13, 109.71, 86.57, 29.52, 23.56, 0.16; IR (neat): $v=2959,2165,1248$, $1115,1007,912,838,797,774,758,654,425 \mathrm{~cm}^{-1} ;$ HRMS: $\mathrm{m} / \mathrm{z}$ (EI) calculated for $\left(\mathrm{C}_{17} \mathrm{H}_{20} \mathrm{Si}\right)$ $[\mathrm{M}]^{+}: 252.1329$, found: 252.1331 .

$[\alpha]_{\mathrm{D}}^{25.9}=1.33\left(c 0.94, \mathrm{CHCl}_{3}\right)$.

HPLC (AD-H, 0.46*25 cm, $5 \mu \mathrm{m}$, hexane/isopropanol $=100 / 0$, flow $0.7 \mathrm{~mL} / \mathrm{min}$, detection at $214 \mathrm{~nm}$ ) retention time $=6.43 \mathrm{~min}$ (major) and $7.39 \mathrm{~min}$ (minor)<smiles>CC#CC(CCl)c1cccc2ccccc12</smiles>

The reaction was conducted on a $0.2 \mathrm{mmol}$ scale according to the general conditions. Column chromatography (PE) yielded product $\mathbf{3 b}(48.5 \mathrm{mg}, 85 \%$ yield, $94 \%$ ee) as colorless oil.

${ }^{1} \mathrm{H}$ NMR $\left(400 \mathrm{MHz}, \mathrm{CDCl}_{3}\right) \delta 8.06(\mathrm{~d}, J=8.4 \mathrm{~Hz}, 1 \mathrm{H}), 7.90(\mathrm{~d}, J=8.0 \mathrm{~Hz}, 1 \mathrm{H}), 7.82(\mathrm{t}, J=$ $8.4 \mathrm{~Hz}, 2 \mathrm{H}), 7.59-7.49(\mathrm{~m}, 3 \mathrm{H}), 4.80(\mathrm{dd}, J=8.4,5.6 \mathrm{~Hz}, 1 \mathrm{H}), 3.90(\mathrm{dd}, J=10.4,5.2 \mathrm{~Hz}, 1 \mathrm{H})$, $3.80(\mathrm{dd}, J=10.0,8.4 \mathrm{~Hz}, 1 \mathrm{H}), 0.24(\mathrm{~s}, 9 \mathrm{H}) ;{ }^{13} \mathrm{C} \mathrm{NMR}\left(100 \mathrm{MHz}, \mathrm{CDCl}_{3}\right) \delta 133.93,133.44$, $130.55,129.15,128.58,126.50,126.32,125.75,125.48,122.43,104.76,89.54,47.92,38.63$, -0.01; IR (neat): $v=2959,1248,1089,1075,979,951,880,859,835,783,757,409 \mathrm{~cm}^{-1}$; HRMS: m/z (EI) calculated for $\left(\mathrm{C}_{17} \mathrm{H}_{19} \mathrm{ClSi}\right)[\mathrm{M}]^{+}: 286.0939$, found: 286.0941 .

$[\alpha]_{\mathrm{D}}^{26.5}=29.88\left(c 0.8, \mathrm{CHCl}_{3}\right)$.

HPLC (AD-H, 0.46*25 cm, $5 \mu \mathrm{m}$, hexane/isopropanol = 100/0, flow $0.7 \mathrm{~mL} / \mathrm{min}$, detection at $214 \mathrm{~nm}$ ) retention time $=7.80 \mathrm{~min}$ (major) and $8.60 \mathrm{~min}$ (minor).

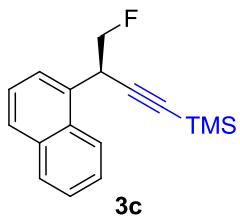


The reaction was conducted on a $0.2 \mathrm{mmol}$ scale according to the general conditions. Column chromatography (PE) yielded product 3c (30.4 mg, 56\% yield, 96\% ee) as colorless oil. 72\% Conversion (alkylarene) was determined by using $\mathrm{CH}_{2} \mathrm{Br}_{2}$ as an internal standard.

${ }^{1} \mathrm{H}$ NMR $\left(400 \mathrm{MHz}, \mathrm{CDCl}_{3}\right) \delta 8.09(\mathrm{~d}, J=8.4 \mathrm{~Hz}, 1 \mathrm{H}), 7.90(\mathrm{~d}, J=8.0 \mathrm{~Hz}, 1 \mathrm{H}), 7.84-7.79(\mathrm{~m}$, 2H), 7.58-7.49 (m, 3H), 4.90-4.83 (m, 1H), 4.76-4.69 (m, 1H), 4.65-4.57 (m, 1H), $0.23(\mathrm{~s}$, $9 \mathrm{H}) ;{ }^{13} \mathrm{C} \mathrm{NMR}\left(100 \mathrm{MHz}, \mathrm{CDCl}_{3}\right) \delta 133.88,132.00(\mathrm{~d}, J=5.8 \mathrm{~Hz}), 130.90,129.05,128.47$, 126.44, 126.40, 125.73, 125.54, 122.59, 103.97 (d, $J=6.4 \mathrm{~Hz}), 89.22,85.42$ (d, $J=179.8 \mathrm{~Hz})$, $36.57(\mathrm{~d}, J=22.5 \mathrm{~Hz}),-0.01 ;{ }^{19} \mathrm{~F}$ NMR $\left(376 \mathrm{MHz}, \mathrm{CDCl}_{3}\right) \delta-211.63(\mathrm{td}, J=47.4,14.7 \mathrm{~Hz}$, 1F); IR (neat): $v=2959,2165,1250,1017,993,905,836,796,778,758,413 \mathrm{~cm}^{-1}$; HRMS: $\mathrm{m} / \mathrm{z}$ (EI) calculated for $\left(\mathrm{C}_{17} \mathrm{H}_{19} \mathrm{FSi}\right)[\mathrm{M}]^{+}: 270.1235$, found: 270.1230 .

$[\alpha]_{\mathrm{D}}^{24.8}=7.71\left(c 0.72, \mathrm{CHCl}_{3}\right)$.

HPLC (AD-H, 0.46*25 cm, $5 \mu \mathrm{m}$, hexane/isopropanol = 100/0, flow $0.7 \mathrm{~mL} / \mathrm{min}$, detection at $214 \mathrm{~nm}$ ) retention time $=8.24 \mathrm{~min}$ (major) and $9.74 \mathrm{~min}$ (minor).

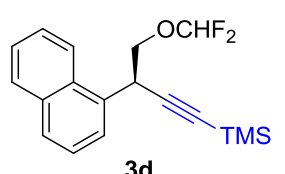

The reaction was conducted on a $0.2 \mathrm{mmol}$ scale according to the general conditions. Column chromatography $(\mathrm{PE} / \mathrm{EA}=50: 1)$ yielded product 3d $(38.0 \mathrm{mg}, 60 \%$ yield, $96 \%$ ee $)$ as colorless oil. $64 \%$ Conversion (alkylarene) was determined by using $\mathrm{CH}_{2} \mathrm{Br}_{2}$ as an internal standard.

${ }^{1} \mathrm{H}$ NMR $\left(400 \mathrm{MHz}, \mathrm{CDCl}_{3}\right) \delta 8.08(\mathrm{~d}, J=8.4 \mathrm{~Hz}, 1 \mathrm{H}), 7.89(\mathrm{~d}, J=7.6 \mathrm{~Hz}, 1 \mathrm{H}), 7.82(\mathrm{~d}, J=$ $8.4 \mathrm{~Hz}, 1 \mathrm{H}), 7.80(\mathrm{~d}, J=7.6 \mathrm{~Hz}, 1 \mathrm{H}), 7.58-7.48(\mathrm{~m}, 3 \mathrm{H}), 6.26(\mathrm{t}, J=74.8 \mathrm{~Hz}, 1 \mathrm{H}), 4.78(\mathrm{dd}$, $J=8.0,6.0 \mathrm{~Hz}, 1 \mathrm{H}), 4.21(\mathrm{dd}, J=10.0,6.0 \mathrm{~Hz}, 1 \mathrm{H}), 4.12(\mathrm{dd}, J=9.6,8.4 \mathrm{~Hz}, 1 \mathrm{H}), 0.22(\mathrm{~s}$, $9 \mathrm{H}) ;{ }^{13} \mathrm{C}$ NMR $\left(100 \mathrm{MHz}, \mathrm{CDCl}_{3}\right) \delta 133.88,132.61,130.78,129.07,128.45,126.46,126.29$, 125.72, 125.51, 122.53, $115.84(\mathrm{t}, J=259.6 \mathrm{~Hz}), 104.73,89.11,66.60(\mathrm{t}, J=5.7 \mathrm{~Hz}), 35.79$, $0.03 ;{ }^{19} \mathrm{~F}$ NMR $\left(376 \mathrm{MHz}, \mathrm{CDCl}_{3}\right) \delta-84.15(\mathrm{~d}, J=73.70 \mathrm{~Hz}, 1 \mathrm{~F}),-84.18(\mathrm{~d}, J=74.1 \mathrm{~Hz}, 1 \mathrm{~F})$; IR (neat): $v=2960,2170,1395,1250,1185,1016,839,798,775,759,652,423 \mathrm{~cm}^{-1}$; HRMS: $\mathrm{m} / \mathrm{z}$ (EI) calculated for $\left(\mathrm{C}_{18} \mathrm{H}_{20} \mathrm{~F}_{2} \mathrm{OSi}\right)[\mathrm{M}]^{+}: 318.1246$, found: 318.1249 .

$[\alpha]_{\mathrm{D}}^{26.9}=21.15\left(c 1.0, \mathrm{CHCl}_{3}\right)$.

HPLC (OD-H, 0.46*25 cm, $5 \mu \mathrm{m}$, hexane/isopropanol = 99/1, flow $0.7 \mathrm{~mL} / \mathrm{min}$, detection at $214 \mathrm{~nm}$ ) retention time $=6.94 \mathrm{~min}$ (minor) and $8.32 \mathrm{~min}$ (major).

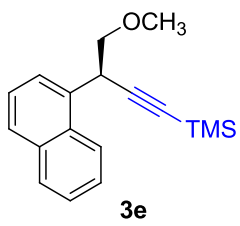


The reaction was conducted on a $0.2 \mathrm{mmol}$ scale according to the general conditions. Column chromatography $(\mathrm{PE} / \mathrm{EA}=40: 1)$ yielded product $3 \mathbf{e}(40.8 \mathrm{mg}, 72 \%$ yield, $92 \%$ ee $)$ as colorless oil.

${ }^{1} \mathrm{H}$ NMR $\left(400 \mathrm{MHz}, \mathrm{CDCl}_{3}\right) \delta 8.14(\mathrm{~d}, J=8.4 \mathrm{~Hz}, 1 \mathrm{H}), 7.89-7.87(\mathrm{~m}, 1 \mathrm{H}), 7.80-7.77(\mathrm{~m}, 2 \mathrm{H})$, 7.56-7.47 (m, 3H), $4.73(\mathrm{dd}, J=8.0,5.6 \mathrm{~Hz}, 1 \mathrm{H}), 3.80-3.71(\mathrm{~m}, 2 \mathrm{H}), 3.41(\mathrm{~s}, 3 \mathrm{H}), 0.22$ (s, $9 \mathrm{H}) ;{ }^{13} \mathrm{C} \mathrm{NMR}\left(100 \mathrm{MHz}, \mathrm{CDCl}_{3}\right) \delta 134.10,133.88,130.93,128.93,127.94,126.08,126.00$, $125.53,125.51,122.97,106.53,87.97,76.74,59.17,36.32,0.08$; IR (neat): $v=2957,2168$, 1248, 1191, 1115, 838, 798, 775, 758, $422 \mathrm{~cm}^{-1}$; HRMS: $\mathrm{m} / \mathrm{z}(\mathrm{EI})$ calculated for $\left(\mathrm{C}_{18} \mathrm{H}_{22} \mathrm{OSi}\right)$ $[\mathrm{M}]^{+}:$282.1434, found: 282.1438 .

$[\alpha]_{\mathrm{D}}^{27.1}=33.64\left(c 0.78, \mathrm{CHCl}_{3}\right)$.

HPLC (OD-H, 0.46*25 cm, $5 \mu \mathrm{m}$, hexane/isopropanol = 100/0, flow $0.7 \mathrm{~mL} / \mathrm{min}$, detection at $214 \mathrm{~nm}$ ) retention time $=44.51 \mathrm{~min}$ (minor) and $69.63 \mathrm{~min}$ (major).<smiles>N#CC(CN)c1cccc2ccccc12</smiles>

The reaction was conducted on a $0.2 \mathrm{mmol}$ scale according to the general conditions. Column chromatography $(\mathrm{PE} / \mathrm{EA}=50: 1)$ yielded product $3 \mathbf{f}(37.7 \mathrm{mg}, 64 \%$ yield, 94\% ee) as colorless oil. $65 \%$ Conversion (alkylarene) was determined by using $\mathrm{CH}_{2} \mathrm{Br}_{2}$ as an internal standard.

${ }^{1} \mathrm{H}$ NMR $\left(400 \mathrm{MHz}, \mathrm{CDCl}_{3}\right) \delta 8.01(\mathrm{~d}, J=8.4 \mathrm{~Hz}, 1 \mathrm{H}), 7.91-7.88(\mathrm{~m}, 1 \mathrm{H}), 7.84-7.81(\mathrm{~m}, 2 \mathrm{H})$, 7.57-7.49 (m, 3H), $4.74(\mathrm{t}, J=6.8 \mathrm{~Hz}, 1 \mathrm{H}), 3.60(\mathrm{~d}, J=7.6 \mathrm{~Hz}, 1 \mathrm{H}), 3.60(\mathrm{~d}, J=5.6 \mathrm{~Hz}, 1 \mathrm{H})$, 0.25 (s, 9H); ${ }^{13} \mathrm{C}$ NMR $\left(100 \mathrm{MHz}, \mathrm{CDCl}_{3}\right) \delta 133.93,133.28,130.41,129.17,128.47,126.47$, $126.21,125.75,125.55,122.29,104.97,90.15,56.13,36.58,-0.12$; IR (neat): $v=2958,2174$, 2096, 1597, 1511, 1315, 1280, 1249, 838, 798, 775, $759 \mathrm{~cm}^{-1}$; HRMS: m/z (EI) calculated for $\left(\mathrm{C}_{17} \mathrm{H}_{19} \mathrm{NSi}\right)\left[\mathrm{M}-\mathrm{N}_{2}\right]^{+}:$265.1281, found: 265.1281 .

$[\alpha]_{\mathrm{D}}^{27.4}=46.68\left(c 0.87, \mathrm{CHCl}_{3}\right)$.

HPLC (OD-H, 0.46*25 cm, $5 \mu \mathrm{m}$, hexane/isopropanol = 99/1, flow $0.7 \mathrm{~mL} / \mathrm{min}$, detection at $214 \mathrm{~nm}$ ) retention time $=7.34 \mathrm{~min}$ (minor) and $8.25 \mathrm{~min}$ (major).

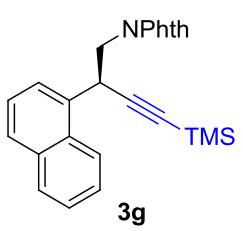

The reaction was conducted on a $0.2 \mathrm{mmol}$ scale according to the general conditions. L9 (19.1 $\mathrm{mg}, 0.048 \mathrm{mmol}, 24 \mathrm{~mol} \%)$, $\mathrm{CuI}$ (7.6 mg, $0.04 \mathrm{mmol}, 20 \mathrm{~mol} \%)$, anhydrous $\mathrm{Na}_{2} \mathrm{CO}_{3}(127.2$ $\mathrm{mg}, 1.2 \mathrm{mmol}), \mathrm{NFSI}(378.4 \mathrm{mg}, 1.2 \mathrm{mmol}, 6.0$ equiv.) and $\mathbf{2 a}(349.4 \mathrm{mg}, 1.6 \mathrm{mmol}, 8.0$ 
equiv.) were used. Column chromatography $(\mathrm{PE} / \mathrm{EA}=4: 1)$ yielded product $\mathbf{3 g}(51.2 \mathrm{mg}, 64 \%$ yield, $90 \%$ ee) as a white solid.

${ }^{1} \mathrm{H}$ NMR $\left(400 \mathrm{MHz}, \mathrm{CDCl}_{3}\right) \delta 8.46(\mathrm{~d}, J=8.4 \mathrm{~Hz}, 1 \mathrm{H}), 7.93-7.87(\mathrm{~m}, 4 \mathrm{H}), 7.82(\mathrm{~d}, J=8.4 \mathrm{~Hz}$, $1 \mathrm{H}), 7.76-7.72(\mathrm{~m}, 2 \mathrm{H}), 7.63-7.59(\mathrm{~m}, 1 \mathrm{H}), 7.54-7.48(\mathrm{~m}, 2 \mathrm{H}), 5.15(\mathrm{dd}, J=10.8,5.2 \mathrm{~Hz}$, $1 \mathrm{H}), 4.17(\mathrm{dd}, J=13.2,10.8 \mathrm{~Hz}, 1 \mathrm{H}), 3.99(\mathrm{dd}, J=13.6,5.2 \mathrm{~Hz}, 1 \mathrm{H}), 0.08(\mathrm{~s}, 9 \mathrm{H}) ;{ }^{13} \mathrm{C} \mathrm{NMR}$ $\left(100 \mathrm{MHz}, \mathrm{CDCl}_{3}\right) \delta 168.12,133.98,133.83,133.37,132.04,130.85,128.87,128.38,126.64$, $126.09,125.74,125.60,123.25,122.98,104.73,89.72,43.48,34.62,-0.30$; IR (neat): $v=$ 2955, 1770, 1713, 1391, 1260, 1247, 1102, 1085, 983, 836, 796, 768, 715, 653, 530, 462, 417 $\mathrm{cm}^{-1}$; HRMS: $\mathrm{m} / \mathrm{z}$ (ESI) calculated for $\left(\mathrm{C}_{25} \mathrm{H}_{24} \mathrm{NO}_{2} \mathrm{Si}\right) \quad[\mathrm{M}+\mathrm{H}]^{+}:$398.1571, found: 398.1575; melting point: $110-112^{\circ} \mathrm{C}$.

$[\alpha]_{\mathrm{D}}^{23.4}=-141.69\left(c 0.86, \mathrm{CHCl}_{3}\right)$.

HPLC (AD-H, 0.46*25 cm, $5 \mu \mathrm{m}$, hexane/isopropanol = 97/3, flow $0.7 \mathrm{~mL} / \mathrm{min}$, detection at $214 \mathrm{~nm}$ ) retention time $=8.46 \mathrm{~min}$ (major) and $8.82 \mathrm{~min}$ (minor).<smiles>CC(=O)CC(C#CCNc1ccccc1)c1cccc2ccccc12</smiles>

The reaction was conducted on a $0.2 \mathrm{mmol}$ scale according to the general conditions. Column chromatography $(\mathrm{PE} / \mathrm{EA}=20: 1)$ yielded product $\mathbf{3 h}(43.5 \mathrm{mg}, 70 \%$ yield, $94 \%$ ee $)$ as colorless oil.

${ }^{1} \mathrm{H}$ NMR $\left(400 \mathrm{MHz}, \mathrm{CDCl}_{3}\right) \delta 8.14(\mathrm{~d}, J=8.4 \mathrm{~Hz}, 1 \mathrm{H}), 7.89-7.86(\mathrm{~m}, 1 \mathrm{H}), 7.78(\mathrm{~d}, J=8.4 \mathrm{~Hz}$, $1 \mathrm{H}), 7.75(\mathrm{~d}, J=7.2 \mathrm{~Hz}, 1 \mathrm{H}), 7.57-7.44(\mathrm{~m}, 3 \mathrm{H}), 4.96(\mathrm{dd}, J=9.6,5.2 \mathrm{~Hz}, 1 \mathrm{H}), 3.72(\mathrm{~s}, 3 \mathrm{H})$, $2.93(\mathrm{dd}, J=15.2,5.2 \mathrm{~Hz}, 1 \mathrm{H}), 2.84(\mathrm{dd}, J=15.2,9.6 \mathrm{~Hz}, 1 \mathrm{H}), 0.18(\mathrm{~s}, 9 \mathrm{H}) ;{ }^{13} \mathrm{C} \mathrm{NMR}(100$ $\left.\mathrm{MHz}, \mathrm{CDCl}_{3}\right) \delta 171.50,135.55,133.97,130.26,129.04,128.06,126.31,125.68,125.54$, 125.32, 122.84, 106.34, 88.54, 51.86, 42.24, 32.06, 0.00; IR (neat): $v=2954,2174,1737$, 1436, 1397, 1348, 1248, 1168, 1149, 838, 800, 775, 759, $637 \mathrm{~cm}^{-1}$; HRMS: m/z (EI) calculated for $\left(\mathrm{C}_{19} \mathrm{H}_{22} \mathrm{O}_{2} \mathrm{Si}\right)[\mathrm{M}]^{+}: 310.1384$, found: 310.1376 .

$[\alpha]_{\mathrm{D}}^{25.7}=12.57\left(c 0.58, \mathrm{CHCl}_{3}\right)$.

HPLC (AD-H, 0.46*25 cm, $5 \mu \mathrm{m}$, hexane/isopropanol = 99/1, flow $0.7 \mathrm{~mL} / \mathrm{min}$, detection at $214 \mathrm{~nm}$ ) retention time $=6.47 \mathrm{~min}$ (minor) and $6.76 \mathrm{~min}$ (major).<smiles>N#CCC(C#CCC(C#N)c1cccc2ccccc12)c1ccccc1</smiles>

The reaction was conducted on a $0.2 \mathrm{mmol}$ scale according to the general conditions. NFSI (189.2 mg, $0.6 \mathrm{mmol}, 3.0$ equiv.) was used. Column chromatography (PE/EA = 25:1) yielded 
product 3i (34.6 mg, 62\% yield, 97\% ee) as colorless oil. 65\% Conversion (alkylarene) was determined by using $\mathrm{CH}_{2} \mathrm{Br}_{2}$ as an internal standard.

${ }^{1} \mathrm{H}$ NMR $\left(400 \mathrm{MHz}, \mathrm{CDCl}_{3}\right) \delta 7.96(\mathrm{~d}, J=8.4 \mathrm{~Hz}, 1 \mathrm{H}), 7.92(\mathrm{~d}, J=7.2 \mathrm{~Hz}, 1 \mathrm{H}), 7.85(\mathrm{t}, J=$ $7.2 \mathrm{~Hz}, 2 \mathrm{H}$ ), 7.60-7.51 (m, 3H), $4.83(\mathrm{dd}, J=7.6,5.6 \mathrm{~Hz}, 1 \mathrm{H}), 3.01(\mathrm{dd}, J=16.4,5.2 \mathrm{~Hz}$, $1 \mathrm{H}), 2.88(\mathrm{dd}, J=16.4,7.6 \mathrm{~Hz}, 1 \mathrm{H}), 0.25(\mathrm{~s}, 9 \mathrm{H}) ;{ }^{13} \mathrm{C} \mathrm{NMR}\left(100 \mathrm{MHz}, \mathrm{CDCl}_{3}\right) \delta 133.96$, $133.18,129.81,129.39$, 128.91, 126.75, 125.89, 125.86, 125.61, 121.76, 117.16, 103.61, 90.87, 32.04, 26.00, -0.13; IR (neat): $v=2960,2184,1597,1510,1413,1250,1076,874$, 840, 804, 778, 762, 613, $423 \mathrm{~cm}^{-1}$; HRMS: $\mathrm{m} / \mathrm{z}$ (EI) calculated for $\left(\mathrm{C}_{18} \mathrm{H}_{19} \mathrm{NSi}\right)[\mathrm{M}]^{+}$: 277.1281, found: 277.1285 .

$[\alpha]_{\mathrm{D}}^{27.9}=45.96\left(c 0.55, \mathrm{CHCl}_{3}\right)$.

HPLC (AD-H, 0.46*25 cm, $5 \mu \mathrm{m}$, hexane/isopropanol = 98/2, flow $0.7 \mathrm{~mL} / \mathrm{min}$, detection at $214 \mathrm{~nm}$ ) retention time $=10.13 \mathrm{~min}$ (major) and $12.39 \mathrm{~min}$ (minor).

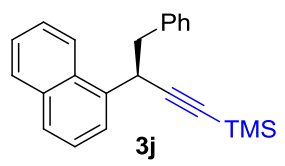

The reaction was conducted on a $0.2 \mathrm{mmol}$ scale according to the general conditions. NFSI (189.2 mg, $0.6 \mathrm{mmol}, 3.0$ equiv.) was used. Column chromatography (PE/EA = 100:1) yielded product $\mathbf{3 j}$ (36.6 mg, 56\% yield, 91\% ee) as colorless oil. 60\% Conversion (alkylarene) was determined by using $\mathrm{CH}_{2} \mathrm{Br}_{2}$ as an internal standard.

${ }^{1} \mathrm{H}$ NMR $\left(400 \mathrm{MHz}, \mathrm{CDCl}_{3}\right) \delta 8.14(\mathrm{~d}, J=8.4 \mathrm{~Hz}, 1 \mathrm{H}), 7.90-7.88(\mathrm{~m}, 1 \mathrm{H}), 7.77(\mathrm{~d}, J=8.4 \mathrm{~Hz}$, $1 \mathrm{H}), 7.66(\mathrm{~d}, J=7.2 \mathrm{~Hz}, 1 \mathrm{H}), 7.57-7.47(\mathrm{~m}, 2 \mathrm{H}), 7.43(\mathrm{t}, J=7.6 \mathrm{~Hz}, 1 \mathrm{H}), 7.29-7.20(\mathrm{~m}, 5 \mathrm{H})$, $4.65(\mathrm{dd}, J=8.8,4.4 \mathrm{~Hz}, 1 \mathrm{H}), 3.22(\mathrm{dd}, J=12.8,4.4 \mathrm{~Hz}, 1 \mathrm{H}), 3.05(\mathrm{dd}, J=13.2,9.2 \mathrm{~Hz}, 1 \mathrm{H})$, 0.17 (s, 9H); ${ }^{13} \mathrm{C}$ NMR (100 MHz, $\left.\mathrm{CDCl}_{3}\right) \delta 139.01,136.59,133.95,130.45,129.56,129.08$, 127.91, 127.65, 126.46, 126.09, 125.65, 125.50, 125.48, 122.80, 107.73, 89.13, 43.45, 37.69, 0.02; IR (neat): $v=2956,2172,1248,837,794,774,758,730,696,496,422, \mathrm{~cm}^{-1}$; HRMS: $\mathrm{m} / \mathrm{z}(\mathrm{EI})$ calculated for $\left(\mathrm{C}_{23} \mathrm{H}_{24} \mathrm{Si}\right)[\mathrm{M}]^{+}: 328.1642$, found: 328.1646 .

$[\alpha]_{\mathrm{D}}^{28.1}=37.15\left(c 0.45, \mathrm{CHCl}_{3}\right)$.

HPLC (OD-H, 0.46*25 cm, $5 \mu \mathrm{m}$, hexane/isopropanol = 99/1, flow $0.7 \mathrm{~mL} / \mathrm{min}$, detection at $214 \mathrm{~nm}$ ) retention time $=6.09 \mathrm{~min}$ (minor) and $7.11 \mathrm{~min}$ (major).

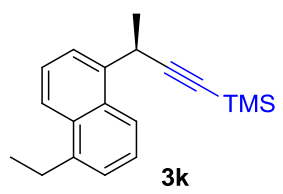

The reaction was conducted on a $0.2 \mathrm{mmol}$ scale according to the general conditions. Column chromatography (PE) yielded product 3k (40.8 mg, 73\% yield, 91\% ee) as colorless oil. (Note: the reaction was monitored by TLC plate until 1,5-diethylnaphthalene was comsumed, and the 
yield (21\%) of $\alpha, \alpha^{6}$-dialkynylation product $\mathbf{3} \mathbf{k}^{\text {' }}$ was determined by ${ }^{1} \mathrm{H}$ NMR spectroscopy of the crude mixture using $\mathrm{CH}_{2} \mathrm{Br}_{2}$ as an internal standard).

${ }^{1} \mathrm{H}$ NMR $\left(400 \mathrm{MHz}, \mathrm{CDCl}_{3}\right) \delta 8.01(\mathrm{~d}, J=8.4 \mathrm{~Hz}, 1 \mathrm{H}), 7.97(\mathrm{~d}, J=8.4 \mathrm{~Hz}, 1 \mathrm{H}), 7.79(\mathrm{~d}, J=$ $6.8 \mathrm{~Hz}, 1 \mathrm{H}), 7.54-7.44(\mathrm{~m}, 2 \mathrm{H}), 7.36(\mathrm{~d}, J=6.8 \mathrm{~Hz}, 1 \mathrm{H}), 4.58(\mathrm{q}, J=6.8 \mathrm{~Hz}, 1 \mathrm{H}), 3.14(\mathrm{q}, J$ $=7.2 \mathrm{~Hz}, 2 \mathrm{H}), 1.64(\mathrm{~d}, J=7.2 \mathrm{~Hz}, 3 \mathrm{H}), 1.40(\mathrm{t}, J=7.6 \mathrm{~Hz}, 3 \mathrm{H}), 0.22(\mathrm{~s}, 9 \mathrm{H}) ;{ }^{13} \mathrm{C} \mathrm{NMR}(100$ $\left.\mathrm{MHz}, \mathrm{CDCl}_{3}\right) \delta 141.09,139.26,132.20,130.74,125.68,125.42,124.63,123.95,123.02$, $121.35,109.91,86.47,29.73,26.39,23.71,15.14,0.16$; IR (neat): $v=2962,2167,1248$, 1097, 1004, 919, 836, 782, 757, 697, 642, $462 \mathrm{~cm}^{-1}$; HRMS: m/z (EI) calculated for $\left(\mathrm{C}_{19} \mathrm{H}_{24} \mathrm{Si}\right)$ $[\mathrm{M}]^{+}: 280.1642$, found: 280.1646 .

$[\alpha]_{\mathrm{D}}^{28.4}=12.55\left(c 0.58, \mathrm{CHCl}_{3}\right)$.

HPLC (OD-H, 0.46*25 cm, $5 \mu \mathrm{m}$, hexane/isopropanol = 99/1, flow $0.7 \mathrm{~mL} / \mathrm{min}$, detection at $254 \mathrm{~nm}$ ) retention time $=5.09 \mathrm{~min}$ (minor) and $5.37 \mathrm{~min}$ (major).

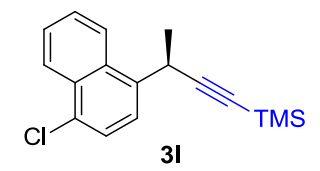

The reaction was conducted on a $0.2 \mathrm{mmol}$ scale according to the general conditions. Column chromatography (PE) yielded product 31 (47.7 mg, 83\% yield, 93\% ee) as colorless oil.

${ }^{1} \mathrm{H}$ NMR (400 MHz, $\left.\mathrm{CDCl}_{3}\right) \delta 8.36-8.34(\mathrm{~m}, 1 \mathrm{H}), 8.13-8.10(\mathrm{~m}, 1 \mathrm{H}), 7.69(\mathrm{~d}, J=8.0 \mathrm{~Hz}, 1 \mathrm{H})$, 7.63-7.57 (m, 3H), $4.52(\mathrm{q}, J=6.8 \mathrm{~Hz}, 1 \mathrm{H}), 1.63(\mathrm{~d}, J=7.2 \mathrm{~Hz}, 3 \mathrm{H}), 0.22(\mathrm{~s}, 9 \mathrm{H}) ;{ }^{13} \mathrm{C} \mathrm{NMR}$ $\left(100 \mathrm{MHz}, \mathrm{CDCl}_{3}\right) \delta 138.01,131.54,131.00,130.95,126.68,126.59,125.94,125.35,124.34$, 123.51, 109.09, 86.98, 29.35, 23.49, 0.11; IR (neat): $v=2958,2168,1509,1381,1248,934$, 888, 836, 754, 698, 651, $420 \mathrm{~cm}^{-1}$; HRMS: m/z (EI) calculated for $\left(\mathrm{C}_{17} \mathrm{H}_{19} \mathrm{ClSi}\right)[\mathrm{M}]^{+}$: 286.0939, found: 286.0944 .

$[\alpha]_{\mathrm{D}}^{28.6}=8.03\left(c 0.97, \mathrm{CHCl}_{3}\right)$

HPLC (OD-H, 0.46*25 cm, $5 \mu \mathrm{m}$, hexane/isopropanol = 100/0, flow $0.7 \mathrm{~mL} / \mathrm{min}$, detection at $214 \mathrm{~nm}$ ) retention time $=14.77 \mathrm{~min}$ (major) and $17.29 \mathrm{~min}$ (minor).

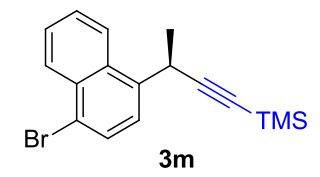

The reaction was conducted on a $0.2 \mathrm{mmol}$ scale according to the general conditions. Column chromatography (PE) yielded product $\mathbf{3 m}(53.9 \mathrm{mg}, 81 \%$ yield, $93 \%$ ee) as colorless oil.

${ }^{1} \mathrm{H} \mathrm{NMR}\left(400 \mathrm{MHz}, \mathrm{CDCl}_{3}\right) \delta 8.32(\mathrm{~d}, J=7.6 \mathrm{~Hz}, 1 \mathrm{H}), 8.10(\mathrm{~d}, J=8.0 \mathrm{~Hz}, 1 \mathrm{H}), 7.80(\mathrm{~d}, J=$ $8.0 \mathrm{~Hz}, 1 \mathrm{H}), 7.64-7.56(\mathrm{~m}, 3 \mathrm{H}), 4.52(\mathrm{q}, J=7.2 \mathrm{~Hz}, 1 \mathrm{H}), 1.63(\mathrm{~d}, J=7.2 \mathrm{~Hz}, 3 \mathrm{H}), 0.22$ (s, 9H); ${ }^{13} \mathrm{C} \mathrm{NMR}\left(100 \mathrm{MHz}, \mathrm{CDCl}_{3}\right) \delta 138.79,132.07,131.68,129.76,128.13,126.89,126.73$, 124.86, 123.51, 121.97, 109.00, 87.00, 29.37, 23.43, 0.10; IR (neat): $v=2958,2163,1592$, 
1507, 1449, 1378, 1247, 1124, 1101, 1009, 920, 877, 862, 830, 751, 655, $426 \mathrm{~cm}^{-1}$; HRMS: $\mathrm{m} / \mathrm{z}(\mathrm{EI})$ calculated for $\left(\mathrm{C}_{17} \mathrm{H}_{19} \mathrm{BrSi}\right)[\mathrm{M}]^{+}: 330.0434$, found: 330.0438 .

$[\alpha]_{\mathrm{D}}^{28.8}=10.54\left(c 1.0, \mathrm{CHCl}_{3}\right)$.

HPLC (OD-H, 0.46*25 cm, $5 \mu \mathrm{m}$, hexane/isopropanol = 100/0, flow $0.7 \mathrm{~mL} / \mathrm{min}$, detection at $214 \mathrm{~nm}$ ) retention time $=17.40 \mathrm{~min}$ (major) and $20.40 \mathrm{~min}$ (minor).

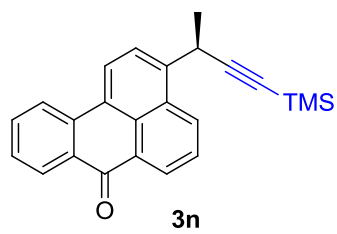

The reaction was conducted on a 0.2 mmol scale according to the general conditions. Column chromatography $(\mathrm{PE} / \mathrm{EA}=15: 1)$ yielded product $3 \mathrm{n}(42.3 \mathrm{mg}, 60 \%$ yield, $95 \%$ ee $)$ as a yellow solid.

${ }^{1} \mathrm{H}$ NMR (400 MHz, $\left.\mathrm{CDCl}_{3}\right) \delta 8.77(\mathrm{~d}, J=8.0 \mathrm{~Hz}, 1 \mathrm{H}), 8.51(\mathrm{~d}, J=8.4 \mathrm{~Hz}, 1 \mathrm{H}), 8.47(\mathrm{~d}, J=$ $8.4 \mathrm{~Hz}, 1 \mathrm{H}), 8.43(\mathrm{~d}, J=8.0 \mathrm{~Hz}, 1 \mathrm{H}), 8.31(\mathrm{~d}, J=8.0 \mathrm{~Hz}, 1 \mathrm{H}), 7.93(\mathrm{~d}, J=7.6 \mathrm{~Hz}, 1 \mathrm{H}), 7.81$ $(\mathrm{t}, J=8.0 \mathrm{~Hz}, 1 \mathrm{H}), 7.72(\mathrm{t}, J=8.0 \mathrm{~Hz}, 1 \mathrm{H}), 7.53(\mathrm{t}, J=8.0 \mathrm{~Hz}, 1 \mathrm{H}), 4.61(\mathrm{q}, J=7.6 \mathrm{~Hz}, 1 \mathrm{H})$, 1.69 (d, $J=7.2 \mathrm{~Hz}, 3 \mathrm{H}), 0.22$ (s, 9H); ${ }^{13} \mathrm{C} \mathrm{NMR}\left(100 \mathrm{MHz}, \mathrm{CDCl}_{3}\right) \delta 183.99,141.54,136.16$, $133.40,130.63,130.23,130.20,129.50,129.16,128.42,128.11,127.99,126.41,125.83$, 125.04, 124.24, 122.91, 108.72, 87.47, 29.81, 23.91, 0.09; IR (neat): $v=2957,2174,1650$, $1577,1313,1245,838,777,762,752,702,632,469 \mathrm{~cm}^{-1}$; HRMS: m/z (EI) calculated for $\left(\mathrm{C}_{24} \mathrm{H}_{22} \mathrm{OSi}\right)[\mathrm{M}]^{+}: 354.1434$, found: 354.1436 ; melting point: $168-170{ }^{\circ} \mathrm{C}$.

$[\alpha]_{\mathrm{D}}^{28.9}=12.64\left(c 0.54, \mathrm{CHCl}_{3}\right)$.

HPLC (AD-H, 0.46*25 cm, $5 \mu \mathrm{m}$, hexane/isopropanol = 98/2, flow $0.7 \mathrm{~mL} / \mathrm{min}$, detection at $214 \mathrm{~nm}$ ) retention time $=11.52 \mathrm{~min}$ (minor) and $13.08 \mathrm{~min}$ (major).

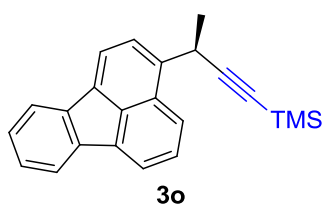

The reaction was conducted on a $0.2 \mathrm{mmol}$ scale according to the general conditions. NFSI (189.2 mg, $0.6 \mathrm{mmol}, 3.0$ equiv.) was used. Column chromatography (PE) yielded product 30 (48.0 mg, 74\% yield, 94\% ee) as a white solid. 82\% Conversion (alkylarene) was determined by using $\mathrm{CH}_{2} \mathrm{Br}_{2}$ as an internal standard.

${ }^{1} \mathrm{H}$ NMR $\left(400 \mathrm{MHz}, \mathrm{CDCl}_{3}\right) \delta 8.07(\mathrm{~d}, J=8.4 \mathrm{~Hz}, 1 \mathrm{H}), 7.93-7.87(\mathrm{~m}, 4 \mathrm{H}), 7.82(\mathrm{~d}, J=7.2 \mathrm{~Hz}$, $1 \mathrm{H}), 7.64(\mathrm{dd}, J=8.4,7.2 \mathrm{~Hz}, 1 \mathrm{H}), 7.39-7.36(\mathrm{~m}, 2 \mathrm{H}), 4.59$ (q, $J=6.8 \mathrm{~Hz}, 1 \mathrm{H}), 1.70(\mathrm{~d}, J=$ $7.2 \mathrm{~Hz}, 3 \mathrm{H}), 0.24(\mathrm{~s}, 9 \mathrm{H}) ;{ }^{13} \mathrm{C} \mathrm{NMR}\left(100 \mathrm{MHz}, \mathrm{CDCl}_{3}\right) \delta 139.87,139.46,139.17,137.50$, $136.05,132.89$, 127.82, 127.71, 127.56, 127.32, 125.98, 123.15, 121.39, 121.27, 120.22, 119.80, 109.56, 86.77, 29.56, 24.79, 0.14; IR (neat): $v=2959,2167,1449,1440,1248,1076$, 
938, 900, 838, 779, 755, $743 \mathrm{~cm}^{-1}$; HRMS: m/z (EI) calculated for $\left(\mathrm{C}_{23} \mathrm{H}_{22} \mathrm{Si}\right)[\mathrm{M}]^{+}: 326.1485$, found: 326.1491 ; melting point: $69-71{ }^{\circ} \mathrm{C}$.

$[\alpha]_{\mathrm{D}}^{29.1}=42.50\left(c 0.60, \mathrm{CHCl}_{3}\right)$.

HPLC (OD-H, 0.46*25 cm, $5 \mu \mathrm{m}$, hexane/isopropanol $=99 / 1$, flow $0.7 \mathrm{~mL} / \mathrm{min}$, detection at $214 \mathrm{~nm}$ ) retention time $=8.81 \mathrm{~min}$ (major) and $11.94 \mathrm{~min}$ (minor).

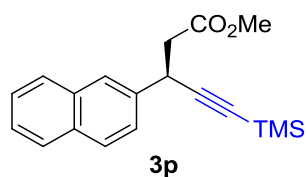

The reaction was conducted on a $0.2 \mathrm{mmol}$ scale according to the general conditions. $\mathrm{CuI}$ ( 7.6 mg, 20 mol\%), L1 (24.5 mg, 24 mol\%), 2 a (349.4 mg, 1.6 mmol, 8.0 equiv.) and NFSI (378.4 $\mathrm{mg}, 1.2 \mathrm{mmol}, 6.0$ equiv.) were used. Column chromatography (PE/EA = 15:1) yielded product 3p (25.0 mg, 40\% yield, $92 \%$ ee) as colorless oil. $43 \%$ Conversion (alkylarene) was determined by using $\mathrm{CH}_{2} \mathrm{Br}_{2}$ as an internal standard.

${ }^{1} \mathrm{H}$ NMR $\left(400 \mathrm{MHz}, \mathrm{CDCl}_{3}\right) \delta 7.83-7.81(\mathrm{~m}, 4 \mathrm{H}), 7.51-7.44(\mathrm{~m}, 3 \mathrm{H}), 4.36(\mathrm{t}, J=8.0 \mathrm{~Hz}, 1 \mathrm{H})$, $3.66(\mathrm{~s}, 3 \mathrm{H}), 2.90(\mathrm{dd}, J=15.2,8.0 \mathrm{~Hz}, 1 \mathrm{H}), 2.81(\mathrm{dd}, J=15.6,7.6 \mathrm{~Hz}, 1 \mathrm{H}), 0.18(\mathrm{~s}, 9 \mathrm{H}) ;{ }^{13} \mathrm{C}$ NMR $\left(100 \mathrm{MHz}, \mathrm{CDCl}_{3}\right) \delta 171.23,137.45,133.35,132.58,128.44,127.84,127.60,126.17$, $125.87,125.47,106.35,88.06,51.79,43.07,35.23,0.02$; IR (neat): $v=2958,2172,1735$, 1435, 1351, 1293, 1019, 839, 812, 799, 760, 739, 704, $472 \mathrm{~cm}^{-1}$; HRMS: m/z (EI) calculated for $\left(\mathrm{C}_{19} \mathrm{H}_{22} \mathrm{O}_{2} \mathrm{Si}\right)[\mathrm{M}]^{+}:$310.1384, found: 310.1391 .

$[\alpha]_{\mathrm{D}}^{29.3}=-2.78\left(c 0.71, \mathrm{CHCl}_{3}\right)$.

HPLC (OD-H, 0.46*25 cm, $5 \mu \mathrm{m}$, hexane/isopropanol = 98/2, flow $0.7 \mathrm{~mL} / \mathrm{min}$, detection at $214 \mathrm{~nm}$ ) retention time $=8.62 \mathrm{~min}$ (minor) and $9.24 \mathrm{~min}$ (major ).

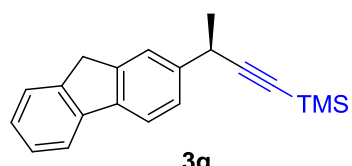

In a dried sealed $10 \mathrm{~mL}$ Schlenk tube, $\mathbf{L 1}(12.3 \mathrm{mg}, 0.024 \mathrm{mmol}, 12 \mathrm{~mol} \%), \mathrm{CuI}$ (3.8 mg, $0.02 \mathrm{mmol}, 10 \mathrm{~mol} \%)$ and anhydrous $\mathrm{Na}_{2} \mathrm{CO}_{3}(63.6 \mathrm{mg}, 0.6 \mathrm{mmol})$ were dissolved in a mixed solvent of $1,2,4,5-\mathrm{C}_{6} \mathrm{H}_{2} \mathrm{~F}_{4}$ and DMA $(4.2 \mathrm{~mL}, v / v=20: 1)$ under $\mathrm{N}_{2}$, and the mixture was stirred for $30 \mathrm{~min}$ and cooled down to $5{ }^{\circ} \mathrm{C}$. Then, 2-ethyl- $9 H$-fluorene $(38.9 \mathrm{mg}, 0.2 \mathrm{mmol}$, 1.0 equiv.), NFSI-2 (230 mg, $0.6 \mathrm{mmol}, 3.0$ equiv.) and $\mathbf{2 a}$ (174.7 mg, $0.8 \mathrm{mmol}, 4.0$ equiv.) were added sequentially, and the tube was sealed with Teflon septum. The reaction mixture was stirred at $5{ }^{\circ} \mathrm{C}$ for $4.5 \mathrm{~d}$. The crude product was purified via a preparative plate $(\mathrm{PE} / \mathrm{EA}=$ $50: 1)$ to yield pure product $\mathbf{3 q}(28.6 \mathrm{mg}, 49 \%$ yield, $86 \%$ ee) as colorless oil.

${ }^{1} \mathrm{H}$ NMR $\left(400 \mathrm{MHz}, \mathrm{CDCl}_{3}\right) \delta 7.77(\mathrm{~d}, J=8.0 \mathrm{~Hz}, 1 \mathrm{H}), 7.74(\mathrm{~d}, J=8.0 \mathrm{~Hz}, 1 \mathrm{H}), 7.57(\mathrm{~s}, 1 \mathrm{H})$, $7.54(\mathrm{~d}, J=7.6 \mathrm{~Hz}, 1 \mathrm{H}), 7.40(\mathrm{~d}, J=7.6 \mathrm{~Hz}, 1 \mathrm{H}), 7.37(\mathrm{t}, J=7.2 \mathrm{~Hz}, 1 \mathrm{H}), 7.29(\mathrm{t}, J=7.2 \mathrm{~Hz}$, 
1H), 3.90(s, 2H), $3.88(\mathrm{q}, J=6.8 \mathrm{~Hz}, 1 \mathrm{H}), 1.54(\mathrm{~d}, J=6.8 \mathrm{~Hz}, 3 \mathrm{H}), 0.22(\mathrm{~s}, 9 \mathrm{H}) ;{ }^{13} \mathrm{C} \mathrm{NMR}$ $\left(100 \mathrm{MHz}, \mathrm{CDCl}_{3}\right) \delta 143.59,143.27,141.77,141.50,140.29,126.68,126.44,125.56,124.96$, 123.54, 119.81. 119.72, 109.74, 86.20, 36.86, 32.92, 24.83, 0.18; IR (neat): $v=2959,2166$, 1455, 1247, 1087, 1013, 879, 839, 762, 735, $421 \mathrm{~cm}^{-1}$; HRMS: m/z (EI) calculated for $\left(\mathrm{C}_{20} \mathrm{H}_{22} \mathrm{Si}\right)[\mathrm{M}]^{+}:$290.1485, found: 290.1493 .

$[\alpha]_{\mathrm{D}}^{29.5}=9.45\left(c 0.65, \mathrm{CHCl}_{3}\right)$.

HPLC (OD-H, 0.46*25 cm, $5 \mu \mathrm{m}$, hexane/isopropanol = 100/0, flow $0.7 \mathrm{~mL} / \mathrm{min}$, detection at $254 \mathrm{~nm}$ ) retention time $=20.59 \mathrm{~min}$ (major) and $27.26 \mathrm{~min}$ (minor).

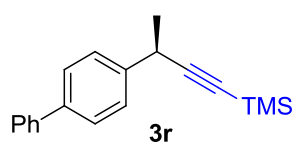

In a dried sealed $10 \mathrm{~mL}$ Schlenk tube, $\mathbf{L 1}(12.3 \mathrm{mg}, 0.024 \mathrm{mmol}, 12 \mathrm{~mol} \%), \mathrm{CuI}$ (3.8 mg, $0.02 \mathrm{mmol}, 10 \mathrm{~mol} \%)$ and anhydrous $\mathrm{Na}_{2} \mathrm{CO}_{3}(63.6 \mathrm{mg}, 0.6 \mathrm{mmol})$ were dissolved in a mixed solvent of $1,2,4,5-\mathrm{C}_{6} \mathrm{H}_{2} \mathrm{~F}_{4}$ and DMA $(2.1 \mathrm{~mL}, v / v=20: 1)$ under $\mathrm{N}_{2}$, and the mixture was stirred for $30 \mathrm{~min}$ and cooled down to $5{ }^{\circ} \mathrm{C}$. Then, 4-ethyl-1,1'-biphenyl (36.5 mg, $0.2 \mathrm{mmol}$, 1.0 equiv.), NFSI-2 (230 mg, $0.6 \mathrm{mmol}, 3.0$ equiv.) and $\mathbf{2 a}(174.7 \mathrm{mg}, 0.8 \mathrm{mmol}, 4.0$ equiv.) were added sequentially, and the tube was sealed with Teflon septum. The reaction mixture was stirred at $5{ }^{\circ} \mathrm{C}$ for $4.5 \mathrm{~d}$. Column chromatography $(\mathrm{PE} / \mathrm{EA}=50: 1)$ yielded product $3 \mathbf{r}$ (36.8 mg, 66\% yield, 90\% ee) as colorless oil. $71 \%$ Conversion (alkylarene) was determined by using $\mathrm{CH}_{2} \mathrm{Br}_{2}$ as an internal standard.

${ }^{1} \mathrm{H}$ NMR (400 MHz, $\left.\mathrm{CDCl}_{3}\right) \delta$ 7.63-7.58 (m, 4H), 7.50-7.45 (m, 4H), $7.37(\mathrm{t}, J=7.6 \mathrm{~Hz}, 1 \mathrm{H})$, $3.87(\mathrm{q}, J=7.2 \mathrm{~Hz}, 1 \mathrm{H}), 1.56(\mathrm{~d}, J=7.2 \mathrm{~Hz}, 3 \mathrm{H}), 0.24(\mathrm{~s}, 9 \mathrm{H}) ;{ }^{13} \mathrm{C} \mathrm{NMR}\left(100 \mathrm{MHz}, \mathrm{CDCl}_{3}\right)$ $\delta 142.11,140.90,139.56,128.72,127.28,127.24,127.13,127.04,109.35,86.29,32.47,24.58$, 0.16; IR (neat): $v=2960,2164,1487,1248,1092,1007,918,835,761,729,697,656,569$, $490 \mathrm{~cm}^{-1}$; HRMS: m/z (EI) calculated for $\left(\mathrm{C}_{19} \mathrm{H}_{22} \mathrm{Si}\right)[\mathrm{M}]^{+}: 278.1485$, found: 278.1490 .

$[\alpha]_{\mathrm{D}}^{29.4}=-0.81\left(c 0.59, \mathrm{CHCl}_{3}\right)$.

HPLC (OD-H, 0.46*25 cm, $5 \mu \mathrm{m}$, hexane / isopropanol = 100/0, flow $0.7 \mathrm{~mL} / \mathrm{min}$, detection at $254 \mathrm{~nm}$ ) retention time $=19.31 \mathrm{~min}$ (major) and $26.02 \mathrm{~min}$ (minor).

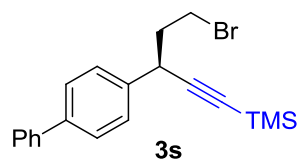

In a dried sealed $10 \mathrm{~mL}$ Schlenk tube, L1 (12.3 mg, $0.024 \mathrm{mmol}, 12 \mathrm{~mol} \%), \mathrm{CuI}$ (3.8 mg, $0.02 \mathrm{mmol}, 10 \mathrm{~mol} \%)$ and anhydrous $\mathrm{Na}_{2} \mathrm{CO}_{3}(63.6 \mathrm{mg}, 0.6 \mathrm{mmol})$ were dissolved in a mixed solvent of $1,2,4,5-\mathrm{C}_{6} \mathrm{H}_{2} \mathrm{~F}_{4}$ and DMA $(2.1 \mathrm{~mL}, v / v=20: 1)$ under $\mathrm{N}_{2}$, and the mixture was stirred for $30 \mathrm{~min}$. Then, 4-(3-bromopropyl)-1,1'-biphenyl (55.1 mg, $0.2 \mathrm{mmol}, 1.0$ equiv.), NFSI (189.2 mg, $0.6 \mathrm{mmol}, 3.0$ equiv.) and $\mathbf{2 a}(174.7 \mathrm{mg}, 0.8 \mathrm{mmol}, 4.0$ equiv.) were added sequentially, and the tube was sealed with Teflon septum. The reaction mixture was stirred at 
room temperature for $60 \mathrm{~h}$. Column chromatography (PE) yielded a mixture of 4-(3bromopropyl)-1,1'-biphenyl and the product 3s. Then, the mxiture was further purified via a preparative plate (PE) to yield product $3 \mathrm{~s}$ ( $40.4 \mathrm{mg}$, $54 \%$ yield, $95 \%$ ee) as a white solid. $74 \%$ Conversion (alkylarene) and $69 \%{ }^{1} \mathrm{H}$ NMR yield (3s) were determined by using $\mathrm{CH}_{2} \mathrm{Br}_{2}$ as an internal standard.

${ }^{1} \mathrm{H}$ NMR $\left(400 \mathrm{MHz}, \mathrm{CDCl}_{3}\right) \delta$ 7.60-7.56 (m, 4H), 7.46-7.42 (m, 4H), 7.37-7.33 (m, 1H), 3.98 $(\mathrm{dd}, J=8.0,6.0 \mathrm{~Hz}, 1 \mathrm{H}), 3.63-3.57(\mathrm{~m}, 1 \mathrm{H}), 3.48-3.43(\mathrm{~m}, 1 \mathrm{H}), 2.34-2.22(\mathrm{~m}, 2 \mathrm{H}), 0.21(\mathrm{~s}$, 9H); ${ }^{13} \mathrm{C}$ NMR (100 MHz, $\left.\mathrm{CDCl}_{3}\right) \delta 140.73,140.09,139.29,128.76,127.87,127.41,127.27$, 127.05, 106.23, 88.43, 41.08, 36.87, 31.11, 0.10; IR (neat): $v=2957,2168,1487,1248,929$, 840, 830, 758, 728, 689, $415 \mathrm{~cm}^{-1}$; HRMS: m/z (EI) calculated for $\left(\mathrm{C}_{20} \mathrm{H}_{23} \mathrm{BrSi}\right)[\mathrm{M}]^{+}$: 370.0747, found: 370.0753 ; melting point: $75-77^{\circ} \mathrm{C}$.

$[\alpha]_{\mathrm{D}}^{29.4}=-17.83\left(c 0.8, \mathrm{CHCl}_{3}\right)$.

HPLC (OD-H, 0.46*25 cm, $5 \mu \mathrm{m}$, hexane/isopropanol = 99/1, flow $0.7 \mathrm{~mL} / \mathrm{min}$, detection at $254 \mathrm{~nm}$ ) retention time $=5.97 \mathrm{~min}$ (major) and $6.49 \mathrm{~min}$ (minor).

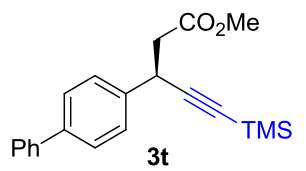

In a dried sealed $10 \mathrm{~mL}$ Schlenk tube, $\mathbf{L 1}(12.3 \mathrm{mg}, 0.024 \mathrm{mmol}, 12 \mathrm{~mol} \%), \mathrm{CuI}$ (3.8 mg, $0.02 \mathrm{mmol}, 10 \mathrm{~mol} \%)$ and anhydrous $\mathrm{Na}_{2} \mathrm{CO}_{3}(63.6 \mathrm{mg}, 0.6 \mathrm{mmol})$ were dissolved in a mixed solvent of $1,2,4,5-\mathrm{C}_{6} \mathrm{H}_{2} \mathrm{~F}_{4}$ and DMA $(4.2 \mathrm{~mL}, v / v=20: 1)$ under $\mathrm{N}_{2}$, and the mixture was stirred for $30 \mathrm{~min}$ and cooled down to $5{ }^{\circ} \mathrm{C}$. Then, methyl 3-([1,1'-biphenyl]-4-yl)propanoate (48.1 mg, $0.2 \mathrm{mmol}, 1.0$ equiv.), NFSI (189.2 mg, $0.6 \mathrm{mmol}, 3.0$ equiv.) and 2a (174.7 mg, $0.8 \mathrm{mmol}, 4.0$ equiv.) were added sequentially, and the tube was sealed with Teflon septum. The reaction mixture was stirred at $5{ }^{\circ} \mathrm{C}$ for $4.5 \mathrm{~d}$. Column chromatography (PE/EA = 10:1) yielded product $3 \mathrm{t}(38.1 \mathrm{mg}, 57 \%$ yield, $97 \%$ ee) as a white solid. $62 \%$ Conversion (alkylarene) was determined by using $\mathrm{CH}_{2} \mathrm{Br}_{2}$ as an internal standard.

${ }^{1} \mathrm{H} \mathrm{NMR}\left(400 \mathrm{MHz}, \mathrm{CDCl}_{3}\right) \delta 7.57(\mathrm{t}, J=8.4 \mathrm{~Hz}, 4 \mathrm{H}), 7.47-7.42(\mathrm{~m}, 4 \mathrm{H}), 7.34(\mathrm{t}, J=7.2 \mathrm{~Hz}$, $1 \mathrm{H}), 4.24(\mathrm{t}, J=7.6 \mathrm{~Hz}, 1 \mathrm{H}), 3.69(\mathrm{~s}, 3 \mathrm{H}), 2.86(\mathrm{dd}, J=15.6,8.4 \mathrm{~Hz}, 1 \mathrm{H}), 2.76(\mathrm{dd}, J=15.2$, $7.2 \mathrm{~Hz}, 1 \mathrm{H}), 0.18(\mathrm{~s}, 9 \mathrm{H}) ;{ }^{13} \mathrm{C}$ NMR $\left(100 \mathrm{MHz}, \mathrm{CDCl}_{3}\right) \delta 171.21,140.68,140.13,139.14$, $128.73,127.81,127.36,127.25,127.02,106.29$, 87.89, 51.75, 43.21, 34.76, 0.01; IR (neat): $v=2954,2174,1736,1487,1452,1359,1248,1201,1152,841,762,689,471 \mathrm{~cm}^{-1}$; HRMS: $\mathrm{m} / \mathrm{z}$ (EI) calculated for $\left(\mathrm{C}_{21} \mathrm{H}_{24} \mathrm{O}_{2} \mathrm{Si}\right)[\mathrm{M}]^{+}: 336.1540$, found: 336.1550 ; melting point: 63-65 ${ }^{\circ} \mathrm{C}$.

$[\alpha]_{\mathrm{D}}^{29.6}=-5.96\left(c 0.67, \mathrm{CHCl}_{3}\right)$.

HPLC (OD-H, 0.46*25 cm, $5 \mu \mathrm{m}$, hexane/isopropanol = 99/1, flow $0.7 \mathrm{~mL} / \mathrm{min}$, detection at $214 \mathrm{~nm}$ ) retention time $=8.52 \mathrm{~min}$ (major) and $9.44 \mathrm{~min}$ (minor). 


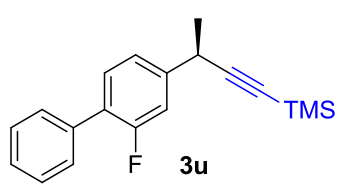

In a dried sealed $10 \mathrm{~mL}$ Schlenk tube, $\mathbf{L 1}$ (24.5 mg, $0.048 \mathrm{mmol}, 24 \mathrm{~mol} \%), \mathrm{CuI}$ (7.6 mg, $0.04 \mathrm{mmol}, 20 \mathrm{~mol} \%)$ and anhydrous $\mathrm{Na}_{2} \mathrm{CO}_{3}(127.2 \mathrm{mg}, 1.2 \mathrm{mmol})$ were dissolved in a mixed solvent of $1,2,4,5-\mathrm{C}_{6} \mathrm{H}_{2} \mathrm{~F}_{4}$ and DMA $(2.1 \mathrm{~mL}, v / v=20: 1)$ under $\mathrm{N}_{2}$, and the mixture was stirred for $30 \mathrm{~min}$. Then, 4-ethyl-2-fluoro-1,1'-biphenyl (40.1 mg, $0.2 \mathrm{mmol}, 1.0$ equiv.), NFSI (378.4 mg, $1.2 \mathrm{mmol}, 6.0$ equiv.) and $2 \mathbf{a}$ (349.4 mg, $1.6 \mathrm{mmol}, 8.0$ equiv.) were added sequentially, and the tube was sealed with Teflon septum. The reaction mixture was stirred at room temperature for $60 \mathrm{~h}$. Column chromatography $(\mathrm{PE} / \mathrm{EA}=50: 1)$ yielded product $3 \mathbf{u}$ (23.9 $\mathrm{mg}, 40 \%$ yield, $79 \%$ ee) as colorless oil. 60\% Conversion (alkylarene) was determined by using $\mathrm{CH}_{2} \mathrm{Br}_{2}$ as an internal standard.

${ }^{1} \mathrm{H}$ NMR $\left(400 \mathrm{MHz}, \mathrm{CDCl}_{3}\right) \delta 7.54(\mathrm{~d}, J=8.4 \mathrm{~Hz}, 2 \mathrm{H}), 7.46-7.34(\mathrm{~m}, 4 \mathrm{H}), 7.20(\mathrm{~d}, J=8.0 \mathrm{~Hz}$, $1 \mathrm{H}), 7.19(\mathrm{~d}, J=11.6 \mathrm{~Hz}, 1 \mathrm{H}), 3.82(\mathrm{q}, J=7.2 \mathrm{~Hz}, 1 \mathrm{H}), 1.52$ (d, $J=7.2 \mathrm{~Hz}, 3 \mathrm{H}), 0.20$ (s, 9H); ${ }^{13} \mathrm{C}$ NMR $\left(100 \mathrm{MHz}, \mathrm{CDCl}_{3}\right) \delta 159.68(\mathrm{~d}, J=247.0 \mathrm{~Hz}), 144.57(\mathrm{~d}, J=7.7 \mathrm{~Hz}), 135.68(\mathrm{~d}, J$ $=1.2 \mathrm{~Hz}), 130.69(\mathrm{~d}, J=3.9 \mathrm{~Hz}), 128.95(\mathrm{~d}, J=2.6 \mathrm{~Hz}), 128.42,127.54,127.23(\mathrm{~d}, J=14.0$ $\mathrm{Hz}), 122.83(\mathrm{~d}, J=3.5 \mathrm{~Hz}), 114.63(\mathrm{~d}, J=23.8 \mathrm{~Hz}), 108.52,86.85,32.32,24.33,0.11 ;{ }^{19} \mathrm{~F}$ NMR (376 MHz, $\left.\mathrm{CDCl}_{3}\right) \delta-117.96-118.01$ (m, 1F); IR (neat): $v=2959,2166,1484,1416$, 1249, 886, 838, 759, 696, $565 \mathrm{~cm}^{-1}$; HRMS: m/z (EI) calculated for $\left(\mathrm{C}_{19} \mathrm{H}_{21} \mathrm{FSi}\right)[\mathrm{M}]^{+}$: 296.1391, found: 296.1397.

$[\alpha]_{\mathrm{D}}^{30.9}=-0.19\left(c 0.72, \mathrm{CHCl}_{3}\right)$.

HPLC (OD-H, 0.46*25 cm, $5 \mu \mathrm{m}$, hexane/isopropanol = 100/0, flow $0.7 \mathrm{~mL} / \mathrm{min}$, detection at $254 \mathrm{~nm}$ ) retention time $=13.45 \mathrm{~min}$ (major) and $17.27 \mathrm{~min}$ (minor).

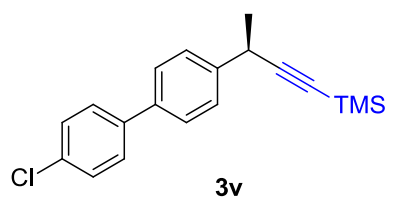

In a dried sealed $10 \mathrm{~mL}$ Schlenk tube, $\mathbf{L 1}$ (12.3 mg, $0.024 \mathrm{mmol}, 12 \mathrm{~mol} \%), \mathrm{CuI}$ (3.8 mg, $0.02 \mathrm{mmol}, 10 \mathrm{~mol} \%)$ and anhydrous $\mathrm{Na}_{2} \mathrm{CO}_{3}(63.6 \mathrm{mg}, 0.6 \mathrm{mmol})$ were dissolved in a mixed solvent of $1,2,4,5-\mathrm{C}_{6} \mathrm{H}_{2} \mathrm{~F}_{4}$ and DMA $(2.1 \mathrm{~mL}, v / v=20: 1)$ under $\mathrm{N}_{2}$, and the mixture was stirred for $30 \mathrm{~min}$ and cooled down to $5{ }^{\circ} \mathrm{C}$. Then, 4-chloro-4'-ethyl-1,1'-biphenyl (43.4 mg, $0.2 \mathrm{mmol}, 1.0$ equiv.), NFSI (189.2 mg, $0.6 \mathrm{mmol}, 3.0$ equiv.) and $\mathbf{2 a}(174.7 \mathrm{mg}, 0.8 \mathrm{mmol}$, 4.0 equiv.) were added sequentially, and the tube was sealed with Teflon septum. The reaction mixture was stirred at $5{ }^{\circ} \mathrm{C}$ for $4.5 \mathrm{~d}$. Column chromatography (PE/EA $\left.=50: 1\right)$ yielded product $3 \mathbf{v}$ ( $38.0 \mathrm{mg}, 61 \%$ yield, $88 \%$ ee) as a white solid. $70 \%$ Conversion (alkylarene) was determined by using $\mathrm{CH}_{2} \mathrm{Br}_{2}$ as an internal standard.

${ }^{1} \mathrm{H}$ NMR (400 MHz, $\left.\mathrm{CDCl}_{3}\right) \delta$ 7.54-7.51 (m, 4H), $7.46(\mathrm{~d}, J=8.0 \mathrm{~Hz}, 2 \mathrm{H}), 7.40(\mathrm{~d}, J=8.4 \mathrm{~Hz}$, $2 \mathrm{H}), 3.84(\mathrm{q}, J=7.2 \mathrm{~Hz}, 1 \mathrm{H}), 1.53(\mathrm{~d}, J=7.6 \mathrm{~Hz}, 3 \mathrm{H}), 0.21(\mathrm{~s}, 9 \mathrm{H}) ;{ }^{13} \mathrm{C}$ NMR $(100 \mathrm{MHz}$, 
$\left.\mathrm{CDCl}_{3}\right) \delta 142.55,139.35,138.32,133.23,128.88,128.26,127.43,127.08,109.22,86.43$, 32.48, 24.53, 0.15; IR (neat): $v=2956,2165,1484,1247,1090,1005,916,837,813,757$ $\mathrm{cm}^{-1}$; HRMS: $\mathrm{m} / \mathrm{z}$ (EI) calculated for $\left(\mathrm{C}_{19} \mathrm{H}_{21} \mathrm{ClSi}\right)[\mathrm{M}]^{+}: 312.1096$, found: 312.1105 ; melting point: $65-67^{\circ} \mathrm{C}$.

$[\alpha]_{\mathrm{D}}^{29.5}=-0.99\left(c 0.63, \mathrm{CHCl}_{3}\right)$.

HPLC (OD-H, 0.46*25 cm, $5 \mu \mathrm{m}$, hexane/isopropanol = 100/0, flow $0.7 \mathrm{~mL} / \mathrm{min}$, detection at $214 \mathrm{~nm}$ ) retention time $=8.60 \mathrm{~min}$ (major) and $9.26 \mathrm{~min}$ (minor).

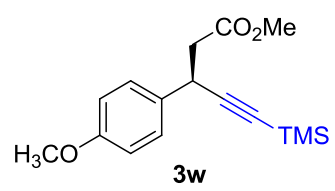

In a dried sealed $10 \mathrm{~mL}$ Schlenk tube, $\mathbf{L 1}(24.5 \mathrm{mg}, 0.048 \mathrm{mmol}, 24 \mathrm{~mol} \%), \mathrm{CuI}$ (7.6 mg, $0.04 \mathrm{mmol}, 20 \mathrm{~mol} \%$ ) and anhydrous $\mathrm{Na}_{2} \mathrm{CO}_{3}$ (127.2 mg, $1.2 \mathrm{mmol}, 6.0$ equiv.) were dissolved in a mixed solvent of $1,2,4,5-\mathrm{C}_{6} \mathrm{H}_{2} \mathrm{~F}_{4}$ and DMA $(2.5 \mathrm{~mL}, v / v=4: 1)$ under $\mathrm{N}_{2}$, and the mixture was stirred for $30 \mathrm{~min}$ and cooled down to $5{ }^{\circ} \mathrm{C}$. Then, methyl 3-(4methoxyphenyl)propanoate ( $38.9 \mathrm{mg}, 0.2 \mathrm{mmol}, 1.0$ equiv.), NFSI (378.4 mg, $1.2 \mathrm{mmol}, 6.0$ equiv.) and $2 \mathrm{a}$ (349.4 mg, $1.6 \mathrm{mmol}, 8.0$ equiv.) were added sequentially, and the tube was sealed with Teflon septum. The reaction mixture was stirred at $5{ }^{\circ} \mathrm{C}$ for $4.5 \mathrm{~d}$. Column chromatography $(\mathrm{PE} / \mathrm{EA}=10: 1)$ yielded product $\mathbf{3 w}(29.3 \mathrm{mg}, 50 \%$ yield, $92 \%$ ee $)$ as colorless oil. $80 \%$ Conversion (alkylarene) was determined by using $\mathrm{CH}_{2} \mathrm{Br}_{2}$ as an internal standard. For the accurate determination of the enatiomeric excess, the TMS goup in $\mathbf{3 w}$ was successful removed using $\mathrm{NH}_{4} \mathrm{~F}$ (8.0 equiv.) and using $\mathrm{MeOH}$ as solvent at room temperature for $36 \mathrm{~h}$.

${ }^{1} \mathrm{H}$ NMR $\left(400 \mathrm{MHz}, \mathrm{CDCl}_{3}\right) \delta 7.29(\mathrm{~d}, J=8.8 \mathrm{~Hz}, 2 \mathrm{H}), 6.85(\mathrm{~d}, J=8.4 \mathrm{~Hz}, 2 \mathrm{H}), 4.13(\mathrm{t}, J=$ $8.0 \mathrm{~Hz}, 1 \mathrm{H}), 3.79(\mathrm{~s}, 3 \mathrm{H}), 3.66(\mathrm{~s}, 3 \mathrm{H}), 2.79(\mathrm{dd}, J=14.8,7.6 \mathrm{~Hz}, 1 \mathrm{H}), 2.68(\mathrm{dd}, J=15.2,7.2$ $\mathrm{Hz}, 1 \mathrm{H}), 0.16(\mathrm{~s}, 9 \mathrm{H}) ;{ }^{13} \mathrm{C} \mathrm{NMR}\left(100 \mathrm{MHz}, \mathrm{CDCl}_{3}\right) \delta 171.27,158.68,132.18,128.41,113.97$, 106.74, 87.51, 55.24, 51.68, 43.44, 34.33, 0.02; IR (neat): $v=2956,2174,1738,1611,1510$, 1246, 1176, 1155, 1028, 838, $759 \mathrm{~cm}^{-1}$; HRMS: m/z (EI) calculated for $\left(\mathrm{C}_{16} \mathrm{H}_{22} \mathrm{O}_{3} \mathrm{Si}\right)[\mathrm{M}]^{+}$: 290.1333, found: 290.1341 .

$[\alpha]_{\mathrm{D}}^{29.7}=-3.16\left(c 0.81, \mathrm{CHCl}_{3}\right)$.

HPLC (OD-H, 0.46*25 cm, $5 \mu \mathrm{m}$, hexane/isopropanol = 98/2, flow $0.7 \mathrm{~mL} / \mathrm{min}$, detection at $214 \mathrm{~nm}$ ) retention time $=13.71 \mathrm{~min}$ (major) and $16.02 \mathrm{~min}$ (minor).

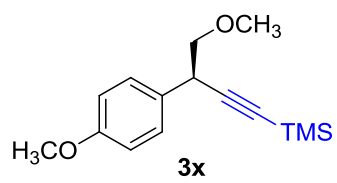

In a dried sealed $10 \mathrm{~mL}$ Schlenk tube, $\mathbf{L 1}$ (12.3 mg, $0.024 \mathrm{mmol}, 12 \mathrm{~mol} \%), \mathrm{CuI}$ (3.8 mg, $0.02 \mathrm{mmol}, 10 \mathrm{~mol} \%)$ and anhydrous $\mathrm{Na}_{2} \mathrm{CO}_{3}(63.6 \mathrm{mg}, 0.6 \mathrm{mmol})$ were dissolved in a mixed 
solvent of $1,2,4,5-\mathrm{C}_{6} \mathrm{H}_{2} \mathrm{~F}_{4}$ and DMA $(2.5 \mathrm{~mL}, v / v=4: 1)$ under $\mathrm{N}_{2}$, and the mixture was stirred for $30 \mathrm{~min}$ and cooled down to $5{ }^{\circ} \mathrm{C}$. Then, 1-methoxy-4-(2-methoxyethyl)benzene $(33.3 \mathrm{mg}$, $0.2 \mathrm{mmol}, 1.0$ equiv.), NFSI (189.2 mg, $0.6 \mathrm{mmol}, 3.0$ equiv.) and $\mathbf{2 a}$ (174.7 mg, $0.8 \mathrm{mmol}$, 4.0 equiv.) were added sequentially, and the tube was sealed with Teflon septum. The reaction mixture was stirred at $5{ }^{\circ} \mathrm{C}$ for $4.5 \mathrm{~d}$. Column chromatography (PE/EA = 10:1) yielded product 3x (27.0 mg, 51\% yield, 92\% ee) as colorless oil. 53\% Conversion (alkylarene) was determined by using $\mathrm{CH}_{2} \mathrm{Br}_{2}$ as an internal standard.

${ }^{1} \mathrm{H}$ NMR $\left(400 \mathrm{MHz}, \mathrm{CDCl}_{3}\right) \delta 7.30(\mathrm{~d}, J=8.8 \mathrm{~Hz}, 2 \mathrm{H}), 6.87(\mathrm{~d}, J=8.4 \mathrm{~Hz}, 2 \mathrm{H}), 3.87(\mathrm{t}, J=$ $7.2 \mathrm{~Hz}, 1 \mathrm{H}), 3.79(\mathrm{~s}, 3 \mathrm{H}), 3.61(\mathrm{dd}, J=9.2,6.8 \mathrm{~Hz}, 1 \mathrm{H}), 3.50(\mathrm{dd}, J=9.2,7.2 \mathrm{~Hz}, 1 \mathrm{H}), 3.35(\mathrm{~s}$, $3 \mathrm{H}), 0.18(\mathrm{~s}, 9 \mathrm{H}) ;{ }^{13} \mathrm{C} \mathrm{NMR}\left(100 \mathrm{MHz}, \mathrm{CDCl}_{3}\right) \delta 158.65,130.72,128.90,113.82,106.04$, 87.65, 77.54, 59.00, 55.22, 38.65, 0.09; IR (neat): $v=2957,2174,1612,1510,1246,1177$, 1114, 1035, 838, $758 \mathrm{~cm}^{-1}$; HRMS: m/z (EI) calculated for $\left(\mathrm{C}_{15} \mathrm{H}_{22} \mathrm{O}_{2} \mathrm{Si}\right)[\mathrm{M}]^{+}: 262.1384$, found: 262.1391 .

$[\alpha]_{\mathrm{D}}^{29.8}=1.03\left(c 0.98, \mathrm{CHCl}_{3}\right)$.

HPLC (AD-H, 0.46*25 cm, $5 \mu \mathrm{m}$, hexane/isopropanol $=99 / 1$, flow $0.7 \mathrm{~mL} / \mathrm{min}$, detection at $214 \mathrm{~nm}$ ) retention time $=6.97 \mathrm{~min}$ (minor) and $7.67 \mathrm{~min}$ (major).

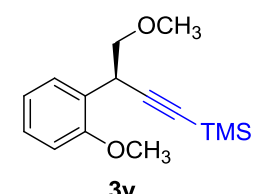

In a dried sealed $10 \mathrm{~mL}$ Schlenk tube, $\mathbf{L 9}(9.6 \mathrm{mg}, 0.024 \mathrm{mmol}, 12 \mathrm{~mol} \%)$, CuI (3.8 mg, 0.02 mmol, $10 \mathrm{~mol} \%)$ and anhydrous $\mathrm{Na}_{2} \mathrm{CO}_{3}(63.6 \mathrm{mg}, 0.6 \mathrm{mmol})$ were dissolved in a mixed solvent of $1,2,4,5-\mathrm{C}_{6} \mathrm{H}_{2} \mathrm{~F}_{4}$ and DMA $(2.1 \mathrm{~mL}, v / v=20: 1)$ under $\mathrm{N}_{2}$, and the mixture was stirred for $30 \mathrm{~min}$ and cooled down to $5{ }^{\circ} \mathrm{C}$. Then, 1-methoxy-2-(2-methoxyethyl)benzene (33.3 mg, $0.2 \mathrm{mmol}, 1.0$ equiv.), NFSI (189.2 mg, $0.6 \mathrm{mmol}, 3.0$ equiv.) and 2a (174.7 mg, $0.8 \mathrm{mmol}, 4.0$ equiv.) were added sequentially, and the tube was sealed with Teflon septum. The reaction mixture was stirred at $5{ }^{\circ} \mathrm{C}$ for $4.5 \mathrm{~d}$. Column chromatography (PE/EA $=10: 1$ ) yielded product $3 \mathbf{y}(28.6 \mathrm{mg}, 54 \%$ yield, $92 \%$ ee) as colorless oil. $57 \%$ Conversion (alkylarene) was determined by using $\mathrm{CH}_{2} \mathrm{Br}_{2}$ as an internal standard.

${ }^{1} \mathrm{H}$ NMR $\left(400 \mathrm{MHz}, \mathrm{CDCl}_{3}\right) \delta 7.55(\mathrm{~d}, J=7.6 \mathrm{~Hz}, 1 \mathrm{H}), 7.22(\mathrm{t}, J=7.6 \mathrm{~Hz}, 1 \mathrm{H}), 6.96(\mathrm{t}, J=$ $7.6 \mathrm{~Hz}, 1 \mathrm{H}), 6.84(\mathrm{~d}, J=8.0 \mathrm{~Hz}, 1 \mathrm{H}), 4.33(\mathrm{dd}, J=6.8,5.2 \mathrm{~Hz}, 1 \mathrm{H}), 3.82$ (s, 3H), 3.55 (dd, $J$ = 9.2, $5.2 \mathrm{~Hz}, 1 \mathrm{H}), 3.42(\mathrm{dd}, J=9.2,6.8 \mathrm{~Hz}, 1 \mathrm{H}), 3.40(\mathrm{~s}, 3 \mathrm{H}), 0.19(\mathrm{~s}, 9 \mathrm{H}) ;{ }^{13} \mathrm{C} \mathrm{NMR}(100$ $\left.\mathrm{MHz}, \mathrm{CDCl}_{3}\right) \delta 156.34,129.07,128.27,126.46,120.66,110.32,106.85,86.74,76.02,58.98$, 55.39, 32.92, 0.15; IR (neat): $v=2957,2172,1600,1588,1492,1462,1439,1243,1114$, 1029, 838, 751, 698, $656 \mathrm{~cm}^{-1}$; HRMS: m/z (EI) calculated for $\left(\mathrm{C}_{15} \mathrm{H}_{22} \mathrm{O}_{2} \mathrm{Si}\right)[\mathrm{M}]^{+}: 262.1384$, found: 262.1386 .

$[\alpha]_{\mathrm{D}}^{29.9}=23.78\left(c 0.55, \mathrm{CHCl}_{3}\right)$. 
HPLC (AD-H, 0.46*25 cm, $5 \mu \mathrm{m}$, hexane/isopropanol = 99/1, flow $0.7 \mathrm{~mL} / \mathrm{min}$, detection at $214 \mathrm{~nm}$ ) retention time $=5.32 \mathrm{~min}$ (minor) and $5.98 \mathrm{~min}$ (major).

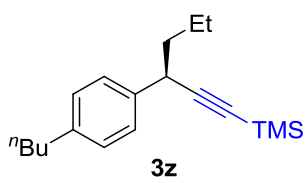

In a dried sealed $10 \mathrm{~mL}$ Schlenk tube, $\mathbf{L 1}$ (12.3 mg, $0.024 \mathrm{mmol}, 12 \mathrm{~mol} \%), \mathrm{CuI}$ (3.8 mg, $0.02 \mathrm{mmol}, 10 \mathrm{~mol} \%)$ and anhydrous $\mathrm{Na}_{2} \mathrm{CO}_{3}(63.6 \mathrm{mg}, 0.6 \mathrm{mmol})$ were dissolved in a mixed solvent of $1,2,4,5-\mathrm{C}_{6} \mathrm{H}_{2} \mathrm{~F}_{4}$ and DEAc ( $N, N$-diethylacetamide) $(2.5 \mathrm{~mL}, v / v=4: 1)$ under $\mathrm{N}_{2}$, and the mixture was stirred for $30 \mathrm{~min}$. Then, 1,4-dibutylbenzene $(38.1 \mathrm{mg}, 0.2 \mathrm{mmol}, 1.0$ equiv.), NFSI (189.2 mg, $0.6 \mathrm{mmol}, 3.0$ equiv.) and 2a (174.7 mg, $0.8 \mathrm{mmol}, 4.0$ equiv.) were added sequentially, and the tube was sealed with Teflon septum. The reaction mixture was stirred at room temperature for $60 \mathrm{~h}$. Column chromatography (PE) yielded product $\mathbf{3 z}$ (22.7 $\mathrm{mg}, 40 \%$ yield, $94 \%$ ee) as colorless oil. For the determination of the enatiomeric excess, the TMS goup in $\mathbf{3 z}$ was successful removed using $\mathrm{K}_{2} \mathrm{CO}_{3}$ (3.0 equiv.) and using $\mathrm{MeOH}$ as solvent at room temperature for $36 \mathrm{~h}$.

${ }^{1} \mathrm{H}$ NMR (400 MHz, $\left.\mathrm{CDCl}_{3}\right) \delta 7.24(\mathrm{~d}, J=8.0 \mathrm{~Hz}, 2 \mathrm{H}), 7.12(\mathrm{~d}, J=8.0 \mathrm{~Hz}, 2 \mathrm{H}), 3.61(\mathrm{dd}, J=$ 8.0, $6.8 \mathrm{~Hz}, 1 \mathrm{H}), 2.58(\mathrm{t}, J=7.6 \mathrm{~Hz}, 2 \mathrm{H}), 1.74-1.55(\mathrm{~m}, 4 \mathrm{H}), 1.51-1.31(\mathrm{~m}, 4 \mathrm{H}), 0.94-0.89(\mathrm{~m}$, $6 \mathrm{H}), 0.17(\mathrm{~s}, 9 \mathrm{H}) ;{ }^{13} \mathrm{C}$ NMR $\left(100 \mathrm{MHz}, \mathrm{CDCl}_{3}\right) \delta 141.09,139.20,128.37,127.26,108.82$, $86.73,40.87,38.15,35.23,33.64,22.39,20.46,13.95,13.77,0.19$; IR (neat): $v=2957,2930$, 2170, 1511, 1459, 1259, 1249, 1093, 1019, 870, 839, 803, $759 \mathrm{~cm}^{-1}$; HRMS: m/z (EI) calculated for $\left(\mathrm{C}_{19} \mathrm{H}_{30} \mathrm{Si}\right)[\mathrm{M}]^{+}: 286.2111$, found: 286.2115 .

$[\alpha]_{\mathrm{D}}^{30.0}=3.34\left(c 0.63, \mathrm{CHCl}_{3}\right)$.

HPLC (OD-H, 0.46*25 cm, $5 \mu \mathrm{m}$, hexane/isopropanol = 100/0, flow $0.7 \mathrm{~mL} / \mathrm{min}$, detection at $214 \mathrm{~nm}$ ) retention time $=8.08 \mathrm{~min}$ (major) and $8.80 \mathrm{~min}$ (minor).

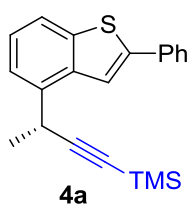

In a dried sealed $10 \mathrm{~mL}$ Schlenk tube, $\mathbf{L 9}$ (9.6 mg, $0.024 \mathrm{mmol}, 12 \mathrm{~mol} \%)$, CuI (3.8 mg, 0.02 mmol, $10 \mathrm{~mol} \%)$ and anhydrous $\mathrm{Na}_{2} \mathrm{CO}_{3}(63.6 \mathrm{mg}, 0.6 \mathrm{mmol})$ were dissolved in a mixed solvent of $1,2,4,5-\mathrm{C}_{6} \mathrm{H}_{2} \mathrm{~F}_{4}$ and DMA $(2.1 \mathrm{~mL}, v / v=20: 1)$ under $\mathrm{N}_{2}$, and the mixture was stirred for $30 \mathrm{~min}$. Then, 4-ethyl-2-phenylbenzo[b]thiophene (47.7 mg, $0.2 \mathrm{mmol}, 1.0$ equiv.), NFSI (189.2 mg, $0.6 \mathrm{mmol}, 3.0$ equiv.) and $\mathbf{2 a}(174.7 \mathrm{mg}, 0.8 \mathrm{mmol}, 4.0$ equiv.) were added sequentially, and the tube was sealed with Teflon septum. The reaction mixture was stirred at room temperature for $60 \mathrm{~h}$. Column chromatography $(\mathrm{PE} / \mathrm{EA}=100: 1)$ yielded product $4 \mathbf{a}$ (31.7 mg, 47\% yield, 91\% ee) as colorless oil. 79\% Conversion (alkylarene) was determined by using $\mathrm{CH}_{2} \mathrm{Br}_{2}$ as an internal standard. 
${ }^{1} \mathrm{H}$ NMR $\left(400 \mathrm{MHz}, \mathrm{CDCl}_{3}\right) \delta 7.77$ (s, $\left.1 \mathrm{H}\right), 7.74-7.71(\mathrm{~m}, 3 \mathrm{H}), 7.46-7.41(\mathrm{~m}, 3 \mathrm{H})$, 7.36-7.28 $(\mathrm{m}, 2 \mathrm{H}), 4.26(\mathrm{q}, J=7.2 \mathrm{~Hz}, 1 \mathrm{H}), 1.62(\mathrm{~d}, J=7.2 \mathrm{~Hz}, 3 \mathrm{H}), 0.19(\mathrm{~s}, 9 \mathrm{H}) ;{ }^{13} \mathrm{C}$ NMR $(100 \mathrm{MHz}$, $\left.\mathrm{CDCl}_{3}\right) \delta 144.00,140.10,138.18,137.58,134.30,128.93,128.28,126.47,124.58,122.53$, 120.88, 117.20, 109.14, 86.53, 31.37, 23.49, 0.12; IR (neat): $v=2957,2167,1488,1459$, $1417,1248,1073,925,837,755,687,640 \mathrm{~cm}^{-1}$; HRMS: $\mathrm{m} / \mathrm{z}$ (EI) calculated for $\left(\mathrm{C}_{21} \mathrm{H}_{22} \mathrm{SSi}\right)$ $[\mathrm{M}]^{+}:$334.1206, found: 334.1211 .

$[\alpha]_{\mathrm{D}}^{29.8}=-26.05\left(c 0.67, \mathrm{CHCl}_{3}\right)$.

HPLC (OD-H, $0.46 * 25 \mathrm{~cm}, 5 \mu \mathrm{m}$, hexane/isopropanol $=100 / 0$, flow $0.7 \mathrm{~mL} / \mathrm{min}$, detection at $214 \mathrm{~nm}$ ) retention time $=29.41 \mathrm{~min}$ (minor) and $32.08 \mathrm{~min}$ (major).

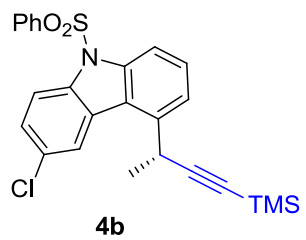

In a dried sealed $10 \mathrm{~mL}$ Schlenk tube, $\mathbf{L 9}(19.1 \mathrm{mg}, 0.048 \mathrm{mmol}, 24 \mathrm{~mol} \%)$, CuI (7.6 mg, $0.04 \mathrm{mmol}, 20 \mathrm{~mol} \%)$ and anhydrous $\mathrm{Na}_{2} \mathrm{CO}_{3}(127.2 \mathrm{mg}, 1.2 \mathrm{mmol})$ were dissolved in a mixed solvent of 1,2,4,5- $\mathrm{C}_{6} \mathrm{H}_{2} \mathrm{~F}_{4}$ and DMA $(2.1 \mathrm{~mL}, v / v=20: 1)$ under $\mathrm{N}_{2}$, and the mixture was stirred for $30 \mathrm{~min}$. Then, 3-chloro-5-ethyl-9-(phenylsulfonyl)- $9 \mathrm{H}$-carbazole (74 $\mathrm{mg}, 0.2$ mmol, 1.0 equiv.), NFSI (378.4 mg, $1.2 \mathrm{mmol}, 6.0$ equiv.) and $\mathbf{2 a}(349.4 \mathrm{mg}, 1.6 \mathrm{mmol}, 8.0$ equiv.) were added sequentially, and the tube was sealed with Teflon septum. The reaction mixture was stirred at room temperature for $60 \mathrm{~h} .48 \%{ }^{1} \mathrm{H}$ NMR yield (4b) was determined by using $\mathrm{CH}_{2} \mathrm{Br}_{2}$ as an internal standard. The product $\mathbf{4 b}$ was purified by RP-HPLC $\left(\mathrm{CH}_{3} \mathrm{CN} / \mathrm{H}_{2} \mathrm{O}=9: 1\right)$ as a white solid. $72 \%$ ee was determined by HPLC on a chiral stationary phase.

${ }^{1} \mathrm{H}$ NMR $\left(400 \mathrm{MHz}, \mathrm{CDCl}_{3}\right) \delta 8.36(\mathrm{~d}, J=8.8 \mathrm{~Hz}, 1 \mathrm{H}), 8.29(\mathrm{~d}, J=8.8 \mathrm{~Hz}, 1 \mathrm{H}), 8.04(\mathrm{~s}, 1 \mathrm{H})$, $7.77(\mathrm{~d}, J=8.4 \mathrm{~Hz}, 2 \mathrm{H}), 7.60(\mathrm{~d}, J=7.2 \mathrm{~Hz}, 1 \mathrm{H}), 7.54-7.45(\mathrm{~m}, 3 \mathrm{H}), 7.33(\mathrm{t}, J=7.6 \mathrm{~Hz}, 2 \mathrm{H})$, $4.48(\mathrm{q}, J=6.8 \mathrm{~Hz}, 1 \mathrm{H}), 1.62(\mathrm{~d}, J=6.8 \mathrm{~Hz}, 3 \mathrm{H}), 0.14(\mathrm{~s}, 9 \mathrm{H}) ;{ }^{13} \mathrm{C} \mathrm{NMR}\left(100 \mathrm{MHz}, \mathrm{CDCl}_{3}\right)$ $\delta 139.23,138.11,137.50,136.83,134.01,129.58,129.12,128.11,126.91,126.89,126.43$, $122.97,122.54,122.31,115.95,113.69,107.63,87.48,30.16,21.81,-0.03$; IR (neat): $v=$ 2960, 2166, 1583, 1446, 1438, 1411, 1373, 1247, 1227, 1213, 1179, 1168, 1130, 1088, 1070, 1018, 935, 903, 839, 758, 722, 681, 594, 585, 566, 554, 535, $476 \mathrm{~cm}^{-1}$; HRMS: $\mathrm{m} / \mathrm{z}$ (EI) calculated for $\left(\mathrm{C}_{25} \mathrm{H}_{24} \mathrm{ClNO}_{2} \mathrm{SSi}\right)[\mathrm{M}]^{+}: 465.0980$, found: 465.0984 ; melting point: $73-75^{\circ} \mathrm{C}$.

$[\alpha]_{\mathrm{D}}^{30.2}=-50.08\left(c 1.0, \mathrm{CHCl}_{3}\right)$.

HPLC (OD-H, $0.46 * 25 \mathrm{~cm}, 5 \mu \mathrm{m}$, hexane/isopropanol $=99 / 1$, flow $0.7 \mathrm{~mL} / \mathrm{min}$, detection at $254 \mathrm{~nm}$ ) retention time $=13.33 \mathrm{~min}$ (major) and $14.22 \mathrm{~min}$ (minor). 


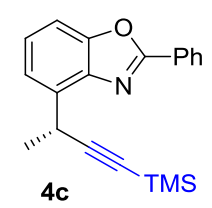

In a dried sealed $10 \mathrm{~mL}$ Schlenk tube, $\mathbf{L 9}$ (19.1 mg, $0.048 \mathrm{mmol}, 24 \mathrm{~mol} \%)$, CuI (7.6 mg, $0.04 \mathrm{mmol}, 20 \mathrm{~mol} \%)$ and anhydrous $\mathrm{Na}_{2} \mathrm{CO}_{3}(127.2 \mathrm{mg}, 1.2 \mathrm{mmol})$ were dissolved in a mixed solvent of 1,2,4,5- $\mathrm{C}_{6} \mathrm{H}_{2} \mathrm{~F}_{4}$ and DMA $(2.1 \mathrm{~mL}, v / v=20: 1)$ under $\mathrm{N}_{2}$, and the mixture was stirred for $30 \mathrm{~min}$ and cooled down to $5{ }^{\circ} \mathrm{C}$. Then, 4-ethyl-2-phenylbenzo[ $d$ ] oxazole (44.7 mg, $0.2 \mathrm{mmol}, 1.0$ equiv.), NFSI (378.4 mg, $1.2 \mathrm{mmol}, 6.0$ equiv.) and 2a (349.4 mg, 1.6 mmol, 8.0 equiv.) were added sequentially, and the tube was sealed with Teflon septum. The reaction mixture was stirred at $5{ }^{\circ} \mathrm{C}$ for $4.5 \mathrm{~d}$. Column chromatography (PE/EA = 30:1) yielded product $4 \mathbf{c}(29.6 \mathrm{mg}, 46 \%$ yield, $90 \%$ ee) as colorless oil. 57\% Conversion (alkylarene) was determined by using $\mathrm{CH}_{2} \mathrm{Br}_{2}$ as an internal standard.

${ }^{1} \mathrm{H}$ NMR (400 MHz, $\left.\mathrm{CDCl}_{3}\right) \delta 8.28-8.23(\mathrm{~m}, 2 \mathrm{H}), 7.56-7.50(\mathrm{~m}, 4 \mathrm{H}), 7.46(\mathrm{~d}, J=7.6 \mathrm{~Hz}, 1 \mathrm{H})$, $7.34(\mathrm{t}, J=7.6 \mathrm{~Hz}, 1 \mathrm{H}), 4.60(\mathrm{q}, J=7.2 \mathrm{~Hz}, 1 \mathrm{H}), 1.65(\mathrm{~d}, J=6.8 \mathrm{~Hz}, 3 \mathrm{H}), 0.21(\mathrm{~s}, 9 \mathrm{H}) ;{ }^{13} \mathrm{C}$ NMR $\left(100 \mathrm{MHz}, \mathrm{CDCl}_{3}\right) \delta 162.34,150.54,139.86,135.28,131.33,128.82,127.65,127.37$, 125.06, 122.18, 109.01, 108.75, 86.24, 28.34, 23.79, 0.19; IR (neat): $v=2958,2169,1623$, 1556, 1488, 1450, 1427, 1245, 1061, 1010, 902, 863, 837, 748, 703, $687 \mathrm{~cm}^{-1}$; HRMS: m/z (ESI) calculated for $\left(\mathrm{C}_{20} \mathrm{H}_{22} \mathrm{NOSi}\right)[\mathrm{M}+\mathrm{H}]^{+}: 320.1465$, found: 320.1470 .

$[\alpha]_{\mathrm{D}}^{30.5}=-54.15\left(c 0.56, \mathrm{CHCl}_{3}\right)$.

HPLC (OD-H, 0.46*25 cm, $5 \mu \mathrm{m}$, hexane/isopropanol = 100/0, flow $0.7 \mathrm{~mL} / \mathrm{min}$, detection at $254 \mathrm{~nm}$ ) retention time $=11.34 \mathrm{~min}$ (minor) and $14.31 \mathrm{~min}$ (major).

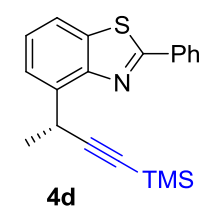

In a dried sealed $10 \mathrm{~mL}$ Schlenk tube, $\mathbf{L 9}(19.1 \mathrm{mg}, 0.048 \mathrm{mmol}, 24 \mathrm{~mol} \%), \mathrm{CuI}$ (7.6 mg, $0.04 \mathrm{mmol}, 20 \mathrm{~mol} \%)$ and anhydrous $\mathrm{Na}_{2} \mathrm{CO}_{3}(127.2 \mathrm{mg}, 1.2 \mathrm{mmol})$ were dissolved in a mixed solvent of 1,2,4,5- $\mathrm{C}_{6} \mathrm{H}_{2} \mathrm{~F}_{4}$ and DMA $(2.1 \mathrm{~mL}, v / v=20: 1)$ under $\mathrm{N}_{2}$, and the mixture was stirred for $30 \mathrm{~min}$. Then, 4-ethyl-2-phenylbenzo[d]thiazole $(47.9 \mathrm{mg}, 0.2 \mathrm{mmol}, 1.0$ equiv.), NFSI (378.4 mg, $1.2 \mathrm{mmol}, 6.0$ equiv.) and 2a (349.4 mg, $1.6 \mathrm{mmol}, 8.0$ equiv.) were added sequentially, and the tube was sealed with Teflon septum. The reaction mixture was stirred at room temperature for $60 \mathrm{~h}$. Column chromatography (PE/EA $=40: 1)$ yielded product $4 \mathbf{d}$ (43.5 mg, 65\% yield, 90\% ee) as a white solid. 83\% Conversion (alkylarene) was determined by using $\mathrm{CH}_{2} \mathrm{Br}_{2}$ as an internal standard.

${ }^{1} \mathrm{H} \mathrm{NMR}\left(400 \mathrm{MHz}, \mathrm{CDCl}_{3}\right) \delta 8.11-8.08(\mathrm{~m}, 2 \mathrm{H}), 7.78(\mathrm{~d}, J=8.0 \mathrm{~Hz}, 1 \mathrm{H}), 7.71(\mathrm{~d}, J=7.2 \mathrm{~Hz}$, $1 \mathrm{H}), 7.52-7.48(\mathrm{~m}, 3 \mathrm{H}), 7.38(\mathrm{t}, J=8.0 \mathrm{~Hz}, 1 \mathrm{H}), 4.92(\mathrm{q}, J=6.8 \mathrm{~Hz}, 1 \mathrm{H}), 1.64(\mathrm{~d}, J=6.8 \mathrm{~Hz}$, $3 \mathrm{H}), 0.21(\mathrm{~s}, 9 \mathrm{H}) ;{ }^{13} \mathrm{C} \mathrm{NMR}\left(100 \mathrm{MHz}, \mathrm{CDCl}_{3}\right) \delta 166.81,151.58,138.04,135.05,133.83$, 
$130.79,128.92,127.51,125.44,124.05,119.87,109.68,86.10,29.38,24.08,0.22$; IR (neat): $v=2956,2171,1479,1409,1249,1104,973,859,841,783,757,684 \mathrm{~cm}^{-1} ;$ HRMS: m/z (ESI) calculated for $\left(\mathrm{C}_{20} \mathrm{H}_{22} \mathrm{NSSi}\right)[\mathrm{M}+\mathrm{H}]^{+}: 336.1237$, found: 336.1241 ; melting point: $99-101$ ${ }^{\circ} \mathrm{C}$

$[\alpha]_{\mathrm{D}}^{31.0}=-67.08\left(c 0.65, \mathrm{CHCl}_{3}\right)$.

HPLC (OD-H, 0.46*25 cm, $5 \mu \mathrm{m}$, hexane/isopropanol = 100/0, flow $0.7 \mathrm{~mL} / \mathrm{min}$, detection at $254 \mathrm{~nm}$ ) retention time $=11.75 \mathrm{~min}$ (minor) and $13.24 \mathrm{~min}$ (major).

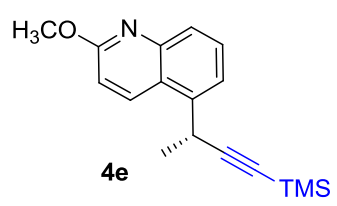

In a dried sealed $10 \mathrm{~mL}$ Schlenk tube, $\mathbf{L 9}$ (19.1 mg, $0.048 \mathrm{mmol}, 24 \mathrm{~mol} \%)$, CuI (7.6 mg, $0.04 \mathrm{mmol}, 20 \mathrm{~mol} \%)$ and anhydrous $\mathrm{Na}_{2} \mathrm{CO}_{3}(127.2 \mathrm{mg}, 1.2 \mathrm{mmol})$ were dissolved in a mixed solvent of $1,2,4,5-\mathrm{C}_{6} \mathrm{H}_{2} \mathrm{~F}_{4}$ and DMA $(2.5 \mathrm{~mL}, v / v=4: 1)$ under $\mathrm{N}_{2}$, and the mixture was stirred for $30 \mathrm{~min}$ and cooled down to $5{ }^{\circ} \mathrm{C}$. Then, 5-ethyl-2-methoxyquinoline (37.5 mg, 0.2 mmol, 1.0 equiv.), NFSI (378.4 mg, $1.2 \mathrm{mmol}, 6.0$ equiv.) and $2 \mathbf{a}$ (349.4 mg, $1.6 \mathrm{mmol}, 8.0$ equiv.) were added sequentially, and the tube was sealed with Teflon septum. The reaction mixture was stirred at $5{ }^{\circ} \mathrm{C}$ for $4.5 \mathrm{~d}$. Column chromatography $(\mathrm{PE} / \mathrm{EA}=60: 1)$ yielded product $4 \mathrm{e}$ (33.8 $\mathrm{mg}, 60 \%$ yield, $92 \%$ ee) as colorless oil. 63\% Conversion (alkylarene) was determined by using $\mathrm{CH}_{2} \mathrm{Br}_{2}$ as an internal standard.

${ }^{1} \mathrm{H}$ NMR $\left(400 \mathrm{MHz}, \mathrm{CDCl}_{3}\right) \delta 8.33(\mathrm{~d}, J=8.8 \mathrm{~Hz}, 1 \mathrm{H}), 7.78-7.76(\mathrm{~m}, 1 \mathrm{H}), 7.61-7.56(\mathrm{~m}, 2 \mathrm{H})$, $6.93(\mathrm{~d}, J=9.2 \mathrm{~Hz}, 1 \mathrm{H}), 4.38(\mathrm{q}, J=7.2 \mathrm{~Hz}, 1 \mathrm{H}), 4.08(\mathrm{~s}, 3 \mathrm{H}), 1.60(\mathrm{~d}, J=7.2 \mathrm{~Hz}, 3 \mathrm{H}), 0.18$ $(\mathrm{s}, 9 \mathrm{H}) ;{ }^{13} \mathrm{C} \mathrm{NMR}\left(100 \mathrm{MHz}, \mathrm{CDCl}_{3}\right) \delta 161.92,147.27,138.96,134.41,129.31,126.65$, $122.35,122.30,112.54,109.20,86.83,53.30,29.59,23.57,0.09$; IR (neat): $v=2957,2169$, 1610, 1578, 1402, 1269, 1249, 1107, 1030, 907, 838, 809, 759, 661, $449 \mathrm{~cm}^{-1}$; HRMS: m/z (ESI) calculated for $\left(\mathrm{C}_{17} \mathrm{H}_{22} \mathrm{NOSi}\right)[\mathrm{M}+\mathrm{H}]^{+}: 284.1465$, found: 284.1470 .

$[\alpha]_{\mathrm{D}}^{30.2}=-16.81\left(c 0.52, \mathrm{CHCl}_{3}\right)$.

HPLC (OD-H, 0.46*25 cm, $5 \mu \mathrm{m}$, hexane/isopropanol = 99/1, flow $0.7 \mathrm{~mL} / \mathrm{min}$, detection at $214 \mathrm{~nm}$ ) retention time $=5.51 \mathrm{~min}$ (minor) and $5.79 \mathrm{~min}$ (major).

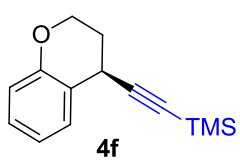

In a dried sealed $10 \mathrm{~mL}$ Schlenk tube, $\mathbf{L 9}(9.6 \mathrm{mg}, 0.024 \mathrm{mmol}, 12 \mathrm{~mol} \%)$, CuI (3.8 mg, 0.02 mmol, $10 \mathrm{~mol} \%)$ and anhydrous $\mathrm{Na}_{2} \mathrm{CO}_{3}(63.6 \mathrm{mg}, 0.6 \mathrm{mmol})$ were dissolved in a mixed solvent of $1,2,4,5-\mathrm{C}_{6} \mathrm{H}_{2} \mathrm{~F}_{4}$ and DMA $(2.5 \mathrm{~mL}, v / v=4: 1)$ under $\mathrm{N}_{2}$, and the mixture was stirred for $30 \mathrm{~min}$ and cooled down to $5{ }^{\circ} \mathrm{C}$. Then, chroman (26.8 mg, $0.2 \mathrm{mmol}, 1.0$ equiv.), NFSI (189.2 mg, $0.6 \mathrm{mmol}, 3.0$ equiv.) and $\mathbf{2 a}(174.7 \mathrm{mg}, 0.8 \mathrm{mmol}, 4.0$ equiv.) were added 
sequentially, and the tube was sealed with Teflon septum. The reaction mixture was stirred at $5{ }^{\circ} \mathrm{C}$ for $4.5 \mathrm{~d}$. Column chromatography (PE) yielded product $\mathbf{4 f}(24.1 \mathrm{mg}, 52 \%$ yield, $87 \%$ ee $)$ as colorless oil. 57\% Conversion (alkylarene) was determined by using $\mathrm{CH}_{2} \mathrm{Br}_{2}$ as an internal standard.

${ }^{1} \mathrm{H}$ NMR $\left(400 \mathrm{MHz}, \mathrm{CDCl}_{3}\right) \delta 7.35(\mathrm{~d}, J=7.6 \mathrm{~Hz}, 1 \mathrm{H}), 7.13(\mathrm{t}, J=7.6 \mathrm{~Hz}, 1 \mathrm{H}), 6.90(\mathrm{t}, J=$ $7.6 \mathrm{~Hz}, 1 \mathrm{H}), 6.79$ (d, $J=8.4 \mathrm{~Hz}, 1 \mathrm{H}), 4.36-4.31(\mathrm{~m}, 1 \mathrm{H}), 4.19-4.13(\mathrm{~m}, 1 \mathrm{H}), 3.86(\mathrm{t}, J=6.4$ $\mathrm{Hz}, 1 \mathrm{H}), 2.25-2.18(\mathrm{~m}, 1 \mathrm{H}), 2.16-2.08(\mathrm{~m}, 1 \mathrm{H}), 0.16(\mathrm{~s}, 9 \mathrm{H}) ;{ }^{13} \mathrm{C} \mathrm{NMR}\left(100 \mathrm{MHz}, \mathrm{CDCl}_{3}\right) \delta$ 153.72, 129.65, 128.30, 121.53, 120.47, 116.85, 107.86, 86.03, 64.36, 29.01, 28.40, 0.06; IR (neat): $v=2958,2169,1607,1582,1488,1453,1310,1267,1248,1224,1070,898,837,750$ $\mathrm{cm}^{-1}$; HRMS: $\mathrm{m} / \mathrm{z}$ (EI) calculated for $\left(\mathrm{C}_{14} \mathrm{H}_{18} \mathrm{OSi}\right)[\mathrm{M}]^{+}: 230.1121$, found: 230.1124 .

$[\alpha]_{\mathrm{D}}^{29.8}=-1.60\left(c 0.68, \mathrm{CHCl}_{3}\right)$.

SFC (OJ-H, 0.46*25 cm, $5 \mu \mathrm{m}$, column T: $40{ }^{\circ} \mathrm{C}$, background press: 2000 psi, $\mathrm{CO}_{2} / \mathrm{MeOH}=$ $95 / 5$, flow $1.0 \mathrm{~mL} / \mathrm{min}$, detection at $214 \mathrm{~nm}$ ) retention time $=9.38 \mathrm{~min}$ (minor) and $9.93 \mathrm{~min}$ (major).

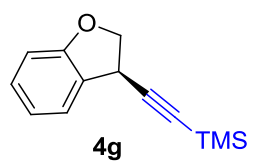

In a dried sealed $10 \mathrm{~mL}$ Schlenk tube, L1 (12.3 mg, $0.024 \mathrm{mmol}, 12 \mathrm{~mol} \%), \mathrm{CuI}$ (3.8 mg, $0.02 \mathrm{mmol}, 10 \mathrm{~mol} \%)$ and anhydrous $\mathrm{Na}_{2} \mathrm{CO}_{3}(63.6 \mathrm{mg}, 0.6 \mathrm{mmol})$ were dissolved in a mixed solvent of $1,2,4,5-\mathrm{C}_{6} \mathrm{H}_{2} \mathrm{~F}_{4}$ and DMA $(2.5 \mathrm{~mL}, v / v=4: 1)$ under $\mathrm{N}_{2}$, and the mixture was stirred for $30 \mathrm{~min}$ and cooled down to $5{ }^{\circ} \mathrm{C}$. Then, 2,3-dihydrobenzofuran $(24 \mathrm{mg}, 0.2 \mathrm{mmol}, 1.0$ equiv.), NFSI (189.2 mg, $0.6 \mathrm{mmol}, 3.0$ equiv.) and $\mathbf{2 a}$ (174.7 mg, $0.8 \mathrm{mmol}, 4.0$ equiv.) were added sequentially, and the tube was sealed with Teflon septum. The reaction mixture was stirred at $5{ }^{\circ} \mathrm{C}$ for $4.5 \mathrm{~d}$. Column chromatography (PE/EA = 100:1) yielded product $\mathbf{4 g}(19.4$ $\mathrm{mg}, 45 \%$ yield, $61 \%$ ee) as colorless oil. $85 \%$ Conversion (alkylarene) was determined by using $\mathrm{CH}_{2} \mathrm{Br}_{2}$ as an internal standard.

${ }^{1} \mathrm{H}$ NMR $\left(400 \mathrm{MHz}, \mathrm{CDCl}_{3}\right) \delta 7.33(\mathrm{~d}, J=7.6 \mathrm{~Hz}, 1 \mathrm{H}), 7.17(\mathrm{t}, J=7.6 \mathrm{~Hz}, 1 \mathrm{H}), 6.93(\mathrm{t}, J=$ $7.6 \mathrm{~Hz}, 1 \mathrm{H}), 6.81(\mathrm{~d}, J=7.6 \mathrm{~Hz}, 1 \mathrm{H}), 4.76(\mathrm{t}, J=12.8 \mathrm{~Hz}, 1 \mathrm{H}), 4.43-4.37(\mathrm{~m}, 2 \mathrm{H}), 0.16$ (s, $9 \mathrm{H}) ;{ }^{13} \mathrm{C}$ NMR $\left(100 \mathrm{MHz}, \mathrm{CDCl}_{3}\right) \delta 159.36,128.87,127.29,124.66,121.04,109.88,104.24$, 87.22, 76.47, 34.80, -0.03; IR (neat): $v=2959,2178,1598,1480,1459,1249,1223,837$, $747 \mathrm{~cm}^{-1}$; HRMS: m/z (EI) calculated for $\left(\mathrm{C}_{13} \mathrm{H}_{16} \mathrm{OSi}\right)[\mathrm{M}]^{+}: 216.0965$, found: 216.0968 .

$[\alpha]_{\mathrm{D}}^{29.9}=-45.94\left(c 0.83, \mathrm{CHCl}_{3}\right)$.

HPLC (OD-H+OD-H, 0.46*25 cm, $5 \mu \mathrm{m}$, hexane/isopropanol $=100 / 0$, flow $0.5 \mathrm{~mL} / \mathrm{min}$, detection at $214 \mathrm{~nm}$ ) retention time $=24.79 \mathrm{~min}$ (minor) and $25.52 \mathrm{~min}$ (major). 


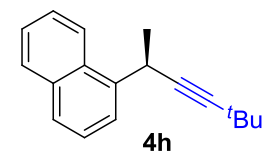

The reaction was conducted on a $0.2 \mathrm{mmol}$ scale according to the general conditions. NFSI (189.2 mg, 0.6 mmol, 3.0 equiv.) was used. Column chromatography (PE) yielded product $\mathbf{4 h}$ (27.7 mg, 59\% yield, 88\% ee) as colorless oil. 83\% Conversion (alkylarene) was determined by using $\mathrm{CH}_{2} \mathrm{Br}_{2}$ as an internal standard.

${ }^{1} \mathrm{H}$ NMR $\left(400 \mathrm{MHz}, \mathrm{CDCl}_{3}\right) \delta 8.12(\mathrm{~d}, J=8.4 \mathrm{~Hz}, 1 \mathrm{H}), 7.88-7.85(\mathrm{~m}, 1 \mathrm{H}), 7.75(\mathrm{t}, J=7.2 \mathrm{~Hz}$, 2H), 7.54-7.45 (m, 3H), $4.49(\mathrm{q}, J=6.8 \mathrm{~Hz}, 1 \mathrm{H}), 1.58(\mathrm{~d}, J=7.2 \mathrm{~Hz}, 3 \mathrm{H}), 1.27(\mathrm{~s}, 9 \mathrm{H}) ;{ }^{13} \mathrm{C}$ NMR $\left(100 \mathrm{MHz}, \mathrm{CDCl}_{3}\right) \delta 139.89,133.91,130.53,128.87,127.15,125.74,125.64,125.33$, 124.16, 123.27, 91.32, 81.43, 31.33, 28.50, 27.48, 24.13; IR (neat): $v=2967,1454,1361$, 1264, 797, 774, 489, $438 \mathrm{~cm}^{-1}$; HRMS: m/z (EI) calculated for $\left(\mathrm{C}_{18} \mathrm{H}_{20}\right)[\mathrm{M}]^{+}:$236.1560, found: 236.1562 .

$[\alpha]_{\mathrm{D}}^{30.1}=11.86\left(c 0.67, \mathrm{CHCl}_{3}\right)$.

HPLC (AD-H, 0.46*25 cm, $5 \mu \mathrm{m}$, hexane/isopropanol = 100/0, flow $0.7 \mathrm{~mL} / \mathrm{min}$, detection at $214 \mathrm{~nm}$ ) retention time $=6.14 \mathrm{~min}$ (major) and $6.72 \mathrm{~min}$ (minor).

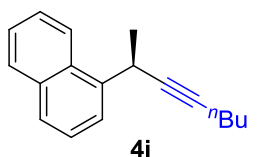

The reaction was conducted on a $0.2 \mathrm{mmol}$ scale according to the general conditions. NFSI (189.2 $\mathrm{mg}, 0.6 \mathrm{mmol}, 3.0$ equiv.) was used and the reaction was performed at $5{ }^{\circ} \mathrm{C}$ for $4.5 \mathrm{~d}$. Column chromatography (PE) yielded product $4 \mathbf{i}$ (20.1 mg, 43\% yield, 91\% ee) as colorless oil. $68 \%$ Conversion (alkylarene) was determined by using $\mathrm{CH}_{2} \mathrm{Br}_{2}$ as an internal standard.

${ }^{1} \mathrm{H}$ NMR $\left(400 \mathrm{MHz}, \mathrm{CDCl}_{3}\right) \delta 8.12(\mathrm{~d}, J=8.4 \mathrm{~Hz}, 1 \mathrm{H}), 7.87-7.85(\mathrm{~m}, 1 \mathrm{H}), 7.76-7.73(\mathrm{~m}, 2 \mathrm{H})$, 7.53-7.44 (m, 3H), 4.52-4.47 (m, 1H), 2.27-2.23 (m, 2H), $1.61(\mathrm{~d}, J=6.8 \mathrm{~Hz}, 3 \mathrm{H}), 1.56-1.39$ $(\mathrm{m}, 4 \mathrm{H}), 0.92(\mathrm{t}, J=6.8 \mathrm{~Hz}, 3 \mathrm{H}) ;{ }^{13} \mathrm{C} \mathrm{NMR}\left(100 \mathrm{MHz}, \mathrm{CDCl}_{3}\right) \delta 139.66,133.93,130.55$, $128.89,127.21,125.81,125.62,125.36,124.08,123.27,83.02,82.62,31.16,28.51,23.76$, 21.97, 18.56, 13.63; IR (neat): $v=2957,2930,2870,1597,1511,1451,797,774,733,573$, 503, $428 \mathrm{~cm}^{-1}$; HRMS: $\mathrm{m} / \mathrm{z}$ (EI) calculated for $\left(\mathrm{C}_{18} \mathrm{H}_{20}\right)[\mathrm{M}]^{+}: 236.1560$, found: 236.1557 .

$[\alpha]_{\mathrm{D}}^{26.4}=-1.01\left(c 0.92, \mathrm{CHCl}_{3}\right)$.

HPLC (AD-H, 0.46*25 cm, $5 \mu \mathrm{m}$, hexane/isopropanol = 100/0, flow $0.7 \mathrm{~mL} / \mathrm{min}$, detection at $214 \mathrm{~nm}$ ) retention time $=8.64 \mathrm{~min}$ (major) and $9.61 \mathrm{~min}$ (minor).

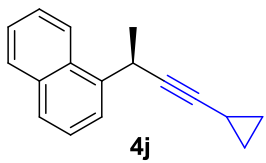


The reaction was conducted on a $0.2 \mathrm{mmol}$ scale according to the general conditions. The reaction in a mixed solvent of $1,2,4,5-\mathrm{C}_{6} \mathrm{H}_{2} \mathrm{~F}_{4}$ and DEAc ( $N, N$-diethylacetamide) $(3.0 \mathrm{~mL}, v / v$ $=2: 1$ ) was performed at $5{ }^{\circ} \mathrm{C}$ for $4.5 \mathrm{~d}$ and NFSI (189.2 mg, $0.6 \mathrm{mmol}, 3.0$ equiv.) was used. Column chromatography (PE) yielded product $4 \mathbf{i}$ (22.9 mg, 52\% yield, $89 \%$ ee) as colorless oil. 89\% Conversion (alkylarene) was determined by using $\mathrm{CH}_{2} \mathrm{Br}_{2}$ as an internal standard.

${ }^{1} \mathrm{H}$ NMR $\left(400 \mathrm{MHz}, \mathrm{CDCl}_{3}\right) \delta 8.09(\mathrm{~d}, J=8.4 \mathrm{~Hz}, 1 \mathrm{H}), 7.85(\mathrm{~d}, J=8.0 \mathrm{~Hz}, 1 \mathrm{H}), 7.73(\mathrm{t}, J=$ $7.2 \mathrm{~Hz}, 2 \mathrm{H}), 7.53-7.43(\mathrm{~m}, 3 \mathrm{H}), 4.46(\mathrm{q}, J=6.8 \mathrm{~Hz}, 1 \mathrm{H}), 1.59(\mathrm{~d}, J=7.2 \mathrm{~Hz}, 3 \mathrm{H}), 1.33-1.26$ $(\mathrm{m}, 1 \mathrm{H}), 0.77-0.71(\mathrm{~m}, 2 \mathrm{H}), 0.69-0.65(\mathrm{~m}, 2 \mathrm{H}) ;{ }^{13} \mathrm{C} \mathrm{NMR}\left(100 \mathrm{MHz}, \mathrm{CDCl}_{3}\right) \delta 139.52$, 133.91, 130.51, 128.89, 127.23, 125.82, 125.62, 125.37, 124.08, 123.20, 85.55, 78.34, 28.41, 23.68, 8.16, 8.13, -0.33; IR (neat): $v=2974,1596,1510,1449,1359,1027,999,797,774$ $\mathrm{cm}^{-1}$; HRMS: $\mathrm{m} / \mathrm{z}$ (EI) calculated for $\left(\mathrm{C}_{17} \mathrm{H}_{16}\right)[\mathrm{M}]^{+}: 220.1247$, found: 220.1244 .

$[\alpha]_{\mathrm{D}}^{26.9}=12.46\left(c 0.34, \mathrm{CHCl}_{3}\right)$.

HPLC (OD-H, 0.46*25 cm, $5 \mu \mathrm{m}$, hexane/isopropanol = 99/1, flow $0.7 \mathrm{~mL} / \mathrm{min}$, detection at $214 \mathrm{~nm}$ ) retention time $=8.12 \mathrm{~min}$ (major) and $12.10 \mathrm{~min}$ (minor).

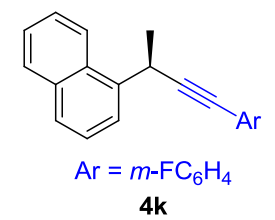

The reaction was conducted on a $0.2 \mathrm{mmol}$ scale according to the general conditions. Column chromatography $(\mathrm{PE} / \mathrm{EA}=100: 1)$ yielded product $\mathbf{4 k}(30.4 \mathrm{mg}, 55 \%$ yield, $91 \%$ ee $)$ as colorless oil.

${ }^{1} \mathrm{H}$ NMR $\left(400 \mathrm{MHz}, \mathrm{CDCl}_{3}\right) \delta 8.14(\mathrm{~d}, J=8.8 \mathrm{~Hz}, 1 \mathrm{H}), 7.87(\mathrm{~d}, J=8.0 \mathrm{~Hz}, 1 \mathrm{H}), 7.77(\mathrm{t}, J=$ $6.8 \mathrm{~Hz}, 2 \mathrm{H}), 7.54(\mathrm{t}, J=8.0 \mathrm{~Hz}, 1 \mathrm{H}), 7.50-7.45(\mathrm{~m}, 2 \mathrm{H}), 7.25-7.19(\mathrm{~m}, 2 \mathrm{H}), 7.14(\mathrm{~d}, J=9.6$ $\mathrm{Hz}, 1 \mathrm{H}), 6.99-6.94(\mathrm{~m}, 1 \mathrm{H}), 4.71(\mathrm{q}, J=7.2 \mathrm{~Hz}, 1 \mathrm{H}), 1.72(\mathrm{~d}, J=7.2 \mathrm{~Hz}, 3 \mathrm{H}) ;{ }^{13} \mathrm{C} \mathrm{NMR}(100$ $\left.\mathrm{MHz}, \mathrm{CDCl}_{3}\right) \delta 162.35(\mathrm{~d}, J=244.8 \mathrm{~Hz}), 138.48,134.00,130.50,129.71(\mathrm{~d}, J=8.9 \mathrm{~Hz})$, 129.01, 127.60, 127.49 (d, $J=2.6 \mathrm{~Hz}), 126.08,125.59$ (d, $J=9.9 \mathrm{~Hz}), 124.24,123.08,118.42$ $(\mathrm{d}, J=22.0 \mathrm{~Hz}), 115.07(\mathrm{~d}, J=21.2 \mathrm{~Hz}), 93.91,81.51(\mathrm{~d}, J=2.9 \mathrm{~Hz}), 29.01,23.16 ;{ }^{19} \mathrm{~F} \mathrm{NMR}$ $\left(376 \mathrm{MHz}, \mathrm{CDCl}_{3}\right) \delta-113.31-113.38(\mathrm{~m}, 1 \mathrm{~F})$; IR (neat): $v=2977,1607,1578,1486,1433$, 1235, 1176, 1150, 868, 798, 774, 734, 681, $504 \mathrm{~cm}^{-1}$; HRMS: m/z (EI) calculated for $\left(\mathrm{C}_{20} \mathrm{H}_{15} \mathrm{~F}\right)[\mathrm{M}]^{+}:$274.1152, found: 274.1155 .

$[\alpha]_{\mathrm{D}}^{30.5}=-76.60\left(c 0.88, \mathrm{CHCl}_{3}\right)$.

HPLC (AD-H, 0.46*25 cm, $5 \mu \mathrm{m}$, hexane/isopropanol = 100/0, flow $0.7 \mathrm{~mL} / \mathrm{min}$, detection at $254 \mathrm{~nm}$ ) retention time $=14.62 \mathrm{~min}$ (major) and $24.17 \mathrm{~min}$ (minor).

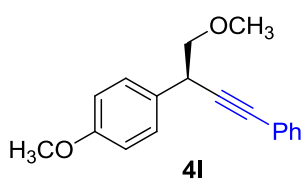


In a dried sealed $10 \mathrm{~mL}$ Schlenk tube, L1 (12.3 mg, $0.024 \mathrm{mmol}, 12 \mathrm{~mol} \%), \mathrm{CuI}$ (3.8 mg, $0.02 \mathrm{mmol}, 10 \mathrm{~mol} \%)$ and anhydrous $\mathrm{Na}_{2} \mathrm{CO}_{3}(63.6 \mathrm{mg}, 0.6 \mathrm{mmol})$ were dissolved in a mixed solvent of $1,2,4,5-\mathrm{C}_{6} \mathrm{H}_{2} \mathrm{~F}_{4}$ and DMA $(2.5 \mathrm{~mL}, v / v=4: 1)$ under $\mathrm{N}_{2}$, and the mixture was stirred for $30 \mathrm{~min}$ and cooled down to $5{ }^{\circ} \mathrm{C}$. Then, 1-methoxy-4-(2-methoxyethyl)benzene (33.3 mg, $0.2 \mathrm{mmol}, \quad 1.0$ equiv.), NFSI (189.2 $\mathrm{mg}, \quad 0.6 \mathrm{mmol}, \quad 3.0$ equiv.) and trimethoxy(phenylethynyl)silane (177.9 $\mathrm{mg}, 0.8 \mathrm{mmol}, 4.0$ equiv.) were added sequentially, and the tube was sealed with Teflon septum. The reaction mixture was stirred at $5{ }^{\circ} \mathrm{C}$ for $4.5 \mathrm{~d}$. Column chromatography (PE/EA = 10:1) yielded product 41 (19.4 mg, 36\% yield, 92\% ee) as colorless oil. 50\% Conversion (alkylarene) was determined by using $\mathrm{CH}_{2} \mathrm{Br}_{2}$ as an internal standard.

${ }^{1} \mathrm{H}$ NMR (400 MHz, $\left.\mathrm{CDCl}_{3}\right) \delta$ 7.47-7.44 (m, 2H), $7.38(\mathrm{~d}, J=8.8 \mathrm{~Hz}, 2 \mathrm{H}), 7.30-7.27(\mathrm{~m}, 3 \mathrm{H})$, $6.89(\mathrm{~d}, J=8.8 \mathrm{~Hz}, 2 \mathrm{H}), 4.07(\mathrm{t}, J=6.8 \mathrm{~Hz}, 1 \mathrm{H}), 3.80(\mathrm{~s}, 3 \mathrm{H}), 3.70(\mathrm{dd}, J=9.2,7.6 \mathrm{~Hz}, 1 \mathrm{H})$, $3.60(\mathrm{dd}, J=9.2,6.8 \mathrm{~Hz}, 1 \mathrm{H}), 3.40(\mathrm{~s}, 3 \mathrm{H}) ;{ }^{13} \mathrm{C} \mathrm{NMR}\left(100 \mathrm{MHz}, \mathrm{CDCl}_{3}\right) \delta 158.70,131.72$, 130.88, 128.94, 128.14, 127.86, 123.44, 113.89, 89.34, 83.43, 77.50, 59.06, 55.26, 38.29; IR (neat): $v=2930,2895,2834,1600,1584,1510,1247,1176,1109,1032,829,756,734,691$, $569,541 \mathrm{~cm}^{-1}$; HRMS: m/z (EI) calculated for $\left(\mathrm{C}_{18} \mathrm{H}_{18} \mathrm{O}_{2}\right)[\mathrm{M}]^{+}:$266.1301, found: 266.1305.

$[\alpha]_{\mathrm{D}}^{30.4}=-13.34\left(c 0.70, \mathrm{CHCl}_{3}\right)$.

HPLC (OD-H, 0.46*25 cm, $5 \mu \mathrm{m}$, hexane/isopropanol = 99/1, flow $0.7 \mathrm{~mL} / \mathrm{min}$, detection at $254 \mathrm{~nm}$ ) retention time $=15.30 \mathrm{~min}$ (major) and $17.27 \mathrm{~min}$ (minor).

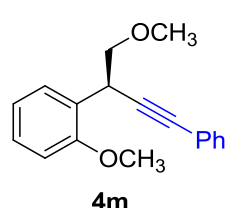

In a dried sealed $10 \mathrm{~mL}$ Schlenk tube, $\mathbf{L 9}(9.6 \mathrm{mg}, 0.024 \mathrm{mmol}, 12 \mathrm{~mol} \%)$, CuI (3.8 mg, 0.02 mmol, $10 \mathrm{~mol} \%)$ and anhydrous $\mathrm{Na}_{2} \mathrm{CO}_{3}(63.6 \mathrm{mg}, 0.6 \mathrm{mmol})$ were dissolved in a mixed solvent of $1,2,4,5-\mathrm{C}_{6} \mathrm{H}_{2} \mathrm{~F}_{4}$ and DMA $(2.1 \mathrm{~mL}, v / v=20: 1)$ under $\mathrm{N}_{2}$, and the mixture was stirred for $30 \mathrm{~min}$ and cooled down to $5{ }^{\circ} \mathrm{C}$. Then, 1-methoxy-2-(2-methoxyethyl)benzene (33.3 mg, $0.2 \mathrm{mmol}, 1.0$ equiv.), NFSI (189.2 mg, $0.6 \mathrm{mmol}, 3.0$ equiv.) and trimethoxy(phenylethynyl)silane (177.9 mg, $0.8 \mathrm{mmol}, 4.0$ equiv.) were added sequentially, and the tube was sealed with Teflon septum. The reaction mixture was stirred at $5{ }^{\circ} \mathrm{C}$ for $4.5 \mathrm{~d}$. Column chromatography $(\mathrm{PE} / \mathrm{EA}=10: 1)$ yielded product $\mathbf{4 m}(16.2 \mathrm{mg}$, 30\% yield, $92 \%$ ee $)$ as colorless oil. 32\% Conversion (alkylarene) was determined by using $\mathrm{CH}_{2} \mathrm{Br}_{2}$ as an internal standard.

${ }^{1} \mathrm{H}$ NMR (400 MHz, $\left.\mathrm{CDCl}_{3}\right) \delta 7.64(\mathrm{~d}, J=7.6 \mathrm{~Hz}, 1 \mathrm{H}), 7.48-7.46(\mathrm{~m}, 2 \mathrm{H}), 7.31-7.24(\mathrm{~m}, 4 \mathrm{H})$, $6.99(\mathrm{t}, J=7.6 \mathrm{~Hz}, 1 \mathrm{H}), 6.88(\mathrm{~d}, J=8.0 \mathrm{~Hz}, 1 \mathrm{H}), 4.62(\mathrm{dd}, J=8.0,6.0 \mathrm{~Hz}, 1 \mathrm{H}), 3.86(\mathrm{~s}, 3 \mathrm{H})$, $3.67(\mathrm{dd}, J=8.8,5.6 \mathrm{~Hz}, 1 \mathrm{H}), 3.59(\mathrm{t}, J=8.0 \mathrm{~Hz}, 1 \mathrm{H}), 3.44(\mathrm{~s}, 3 \mathrm{H}) ;{ }^{13} \mathrm{C} \mathrm{NMR}(100 \mathrm{MHz}$, $\left.\mathrm{CDCl}_{3}\right) \delta 156.38,131.80,129.18,128.35,128.09,127.70,126.67,123.73,120.73,110.43$, 90.06, 82.65, 76.01, 59.00, 55.46, 32.54; IR (neat): $v=2925,2888,2836,1598,1588,1491$, 
1460, 1439, 1242, 1110, 1050, 1026, 751, 691, $503 \mathrm{~cm}^{-1}$; HRMS: m/z (EI) calculated for $\left(\mathrm{C}_{18} \mathrm{H}_{18} \mathrm{O}_{2}\right)[\mathrm{M}]^{+}:$266.1301, found: 266.1301.

$[\alpha]_{\mathrm{D}}^{30.3}=-17.06\left(c 0.65, \mathrm{CHCl}_{3}\right)$.

HPLC (OD-H, 0.46*25 cm, $5 \mu \mathrm{m}$, hexane/isopropanol = 99/1, flow $0.7 \mathrm{~mL} / \mathrm{min}$, detection at $254 \mathrm{~nm}$ ) retention time $=11.93 \mathrm{~min}$ (major) and $22.32 \mathrm{~min}$ (minor).

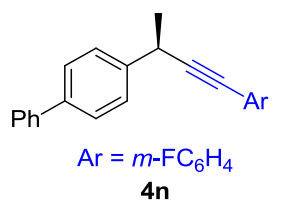

In a dried sealed $10 \mathrm{~mL}$ Schlenk tube, L1 (12.3 mg, $0.024 \mathrm{mmol}, 12 \mathrm{~mol} \%), \mathrm{CuI}$ (3.8 mg, $0.02 \mathrm{mmol}, 10 \mathrm{~mol} \%)$ and anhydrous $\mathrm{Na}_{2} \mathrm{CO}_{3}(63.6 \mathrm{mg}, 0.6 \mathrm{mmol})$ were dissolved in a mixed solvent of $1,2,4,5-\mathrm{C}_{6} \mathrm{H}_{2} \mathrm{~F}_{4}$ and DMA $(2.1 \mathrm{~mL}, v / v=20: 1)$ under $\mathrm{N}_{2}$, and the mixture was stirred for $30 \mathrm{~min}$ and cooled down to $5{ }^{\circ} \mathrm{C}$. Then, 4-ethyl-1,1'-biphenyl (36.5 mg, $0.2 \mathrm{mmol}$, 1.0 equiv.), NFSI-2 (230 $\mathrm{mg}, \quad 0.6 \quad \mathrm{mmol}, \quad 3.0$ equiv.) and ((3fluorophenyl)ethynyl)trimethoxysilane (192.2 $\mathrm{mg}, 0.8 \mathrm{mmol}, 4.0$ equiv.) were added sequentially, and the tube was sealed with Teflon septum. The reaction mixture was stirred at $5{ }^{\circ} \mathrm{C}$ for $4.5 \mathrm{~d}$. Column chromatography (PE/EA = 100:1) yielded product 4n $(24.0 \mathrm{mg}, 40 \%$ yield, $80 \%$ ee) as colorless oil. 59\% Conversion (alkylarene) was determined by using $\mathrm{CH}_{2} \mathrm{Br}_{2}$ as an internal standard.

${ }^{1} \mathrm{H}$ NMR (400 MHz, $\left.\mathrm{CDCl}_{3}\right) \delta$ 7.60-7.56 (m, 4H), 7.51-7.49 (m, 2H), $7.44(\mathrm{t}, J=7.6 \mathrm{~Hz}, 2 \mathrm{H})$, 7.36-7.32 (m, 1H), 7.29-7.22 (m, 2H), 7.17-7.14 (m, 1H), 7.02-6.97 (m, 1H), $4.02(\mathrm{q}, J=7.2$ $\mathrm{Hz}, 1 \mathrm{H}), 1.62(\mathrm{~d}, J=7.2 \mathrm{~Hz}, 3 \mathrm{H}) ;{ }^{13} \mathrm{C} \mathrm{NMR}\left(100 \mathrm{MHz}, \mathrm{CDCl}_{3}\right) \delta 162.36(\mathrm{~d}, J=244.6 \mathrm{~Hz})$, $142.03,140.86,139.79,129.73(\mathrm{~d}, J=8.9 \mathrm{~Hz}), 128.75,127.51(\mathrm{~d}, J=3.0 \mathrm{~Hz}), 127.37,127.32$, 127.20, 127.07, 125.53 (d, $J=9.1 \mathrm{~Hz}), 118.45(\mathrm{~d}, J=23.0 \mathrm{~Hz}), 115.11(\mathrm{~d}, J=20.8 \mathrm{~Hz}), 93.60$, $81.36(\mathrm{~d}, J=3.4 \mathrm{~Hz}), 32.10,24.26 ;{ }^{19} \mathrm{~F}$ NMR $\left(376 \mathrm{MHz}, \mathrm{CDCl}_{3}\right) \delta-113.30-113.36(\mathrm{~m}, 1 \mathrm{~F})$; IR (neat): $v=2975,1607,1578,1485,1263,1169,1150,836,782,763,731,696,681,570$, $518 \mathrm{~cm}^{-1}$; HRMS: m/z (EI) calculated for $\left(\mathrm{C}_{22} \mathrm{H}_{17} \mathrm{~F}\right)[\mathrm{M}]^{+}: 300.1309$, found: 300.1314 .

$[\alpha]_{\mathrm{D}}^{30.6}=-13.99\left(c 0.52, \mathrm{CHCl}_{3}\right)$.

HPLC (OD-H, 0.46*25 cm, $5 \mu \mathrm{m}$, hexane/isopropanol = 99/1, flow $0.7 \mathrm{~mL} / \mathrm{min}$, detection at $254 \mathrm{~nm}$ ) retention time $=8.89 \mathrm{~min}$ (minor) and $9.57 \mathrm{~min}$ (major). 


\section{Single Crystal X-Ray Diffraction Data (3s, 4d and 8)}

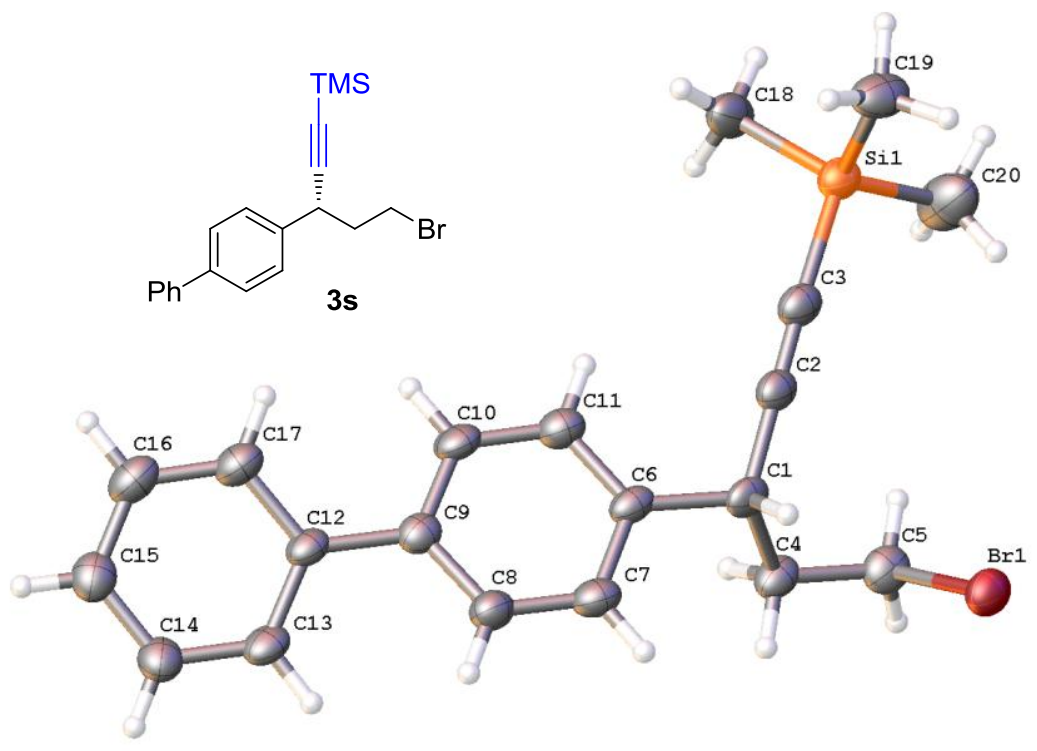

Table 1. Crystal data and structure refinement for mj19476_0m.

Identification code

Empirical formula

Formula weight

Temperature

Wavelength

Crystal system

Space group

Unit cell dimensions

Volume

$\mathrm{Z}$

Density (calculated)

Absorption coefficient

$\mathrm{F}(000)$

Crystal size

Theta range for data collection

Index ranges

Reflections collected

Independent reflections

Completeness to theta $=53.594^{\circ}$

Absorption correction mj19476_0m

C20 H23 Br Si

371.38

$170.01 \mathrm{~K}$

$1.34139 \AA$

Monoclinic

P 1211

$\mathrm{a}=6.2063(10) \AA$

$\alpha=90^{\circ}$.

$\mathrm{b}=8.2335(13) \AA$

$\beta=98.177(9)^{\circ}$.

$\mathrm{c}=18.476(3) \AA$

$\gamma=90^{\circ}$.

934.5(3) $\AA^{3}$

2

$1.320 \mathrm{Mg} / \mathrm{m}^{3}$

$2.416 \mathrm{~mm}^{-1}$

384

$0.1 \times 0.08 \times 0.005 \mathrm{~mm}^{3}$

4.206 to $55.424^{\circ}$.

$-7<=\mathrm{h}<=7,-10<=\mathrm{k}<=10,-22<=1<=19$

8682

$3429[\mathrm{R}(\mathrm{int})=0.1262]$

$98.4 \%$

Semi-empirical from equivalents 
Max. and min. transmission

Refinement method

Data / restraints / parameters

Goodness-of-fit on $\mathrm{F}^{2}$

Final R indices [I $>2 \operatorname{sigma}(\mathrm{I})]$

$\mathrm{R}$ indices (all data)

Absolute structure parameter

Extinction coefficient

Largest diff. peak and hole
0.7508 and 0.3477

Full-matrix least-squares on $\mathrm{F}^{2}$

3429 / 1 / 202

1.122

$\mathrm{R} 1=0.0841, \mathrm{wR} 2=0.2090$

$\mathrm{R} 1=0.1007, \mathrm{wR} 2=0.2314$

$0.07(5)$

$\mathrm{n} / \mathrm{a}$

1.062 and -0.743 e. $\AA^{-3}$

Table 2. Atomic coordinates ( x 10 $)$ and equivalent isotropic displacement parameters $\left(\AA^{2} \times 10^{3}\right)$ for mj19476_0m. U(eq) is defined as one third of the trace of the orthogonalized Uij tensor.

\begin{tabular}{|c|c|c|c|c|}
\hline & $\mathrm{x}$ & $\mathrm{y}$ & $\mathrm{z}$ & $\mathrm{U}(\mathrm{eq})$ \\
\hline $\operatorname{Br}(1)$ & $415(2)$ & $2342(2)$ & $8055(1)$ & $66(1)$ \\
\hline $\operatorname{Si}(1)$ & $7941(5)$ & $6421(4)$ & $8983(2)$ & $56(1)$ \\
\hline $\mathrm{C}(1)$ & $4305(16)$ & $3591(14)$ & $7100(6)$ & $53(2)$ \\
\hline$C(2)$ & $5479(18)$ & $4590(14)$ & $7702(6)$ & $55(2)$ \\
\hline $\mathrm{C}(3)$ & $6432(19)$ & $5369(14)$ & $8188(6)$ & $58(3)$ \\
\hline $\mathrm{C}(4)$ & $4170(20)$ & 1793(13) & $7336(6)$ & $58(3)$ \\
\hline$C(5)$ & $3356(19)$ & $1526(16)$ & $8058(6)$ & $64(3)$ \\
\hline $\mathrm{C}(6)$ & $5364(17)$ & $3717(12)$ & $6404(5)$ & $51(2)$ \\
\hline$C(7)$ & $4240(20)$ & $3023(15)$ & $5757(6)$ & $63(3)$ \\
\hline $\mathrm{C}(8)$ & $5170(20)$ & $3047(15)$ & $5119(6)$ & $59(3)$ \\
\hline $\mathrm{C}(9)$ & 7203(19) & $3746(13)$ & $5091(5)$ & $53(2)$ \\
\hline$C(10)$ & $8260(20)$ & $4427(14)$ & $5739(6)$ & $59(3)$ \\
\hline $\mathrm{C}(11)$ & 7353(19) & $4418(14)$ & $6379(6)$ & $60(3)$ \\
\hline $\mathrm{C}(12)$ & $8233(18)$ & $3731(12)$ & $4418(5)$ & $52(2)$ \\
\hline$C(13)$ & $7430(20)$ & $2754(12)$ & $3819(6)$ & $60(3)$ \\
\hline$C(14)$ & $8430(20)$ & $2699(13)$ & $3190(6)$ & $65(3)$ \\
\hline$C(15)$ & $10220(20)$ & $3635(18)$ & $3140(6)$ & $68(3)$ \\
\hline$C(16)$ & $11010(20)$ & $4630(17)$ & $3720(6)$ & $71(3)$ \\
\hline$C(17)$ & $10010(20)$ & $4714(16)$ & $4340(6)$ & $65(3)$ \\
\hline$C(18)$ & $10870(20)$ & $6510(20)$ & $8905(7)$ & $79(4)$ \\
\hline$C(19)$ & $6930(30)$ & $8499(17)$ & 9012(9) & $86(4)$ \\
\hline$C(20)$ & $7440(30)$ & $5300(30)$ & $9799(7)$ & $108(6)$ \\
\hline
\end{tabular}


Table 3. Bond lengths $[\AA ̊ \AA]$ and angles $\left[^{\circ}\right]$ for mj19476_0m.

\begin{tabular}{|c|c|}
\hline $\operatorname{Br}(1)-C(5)$ & $1.944(12)$ \\
\hline $\operatorname{Si}(1)-C(3)$ & $1.841(11)$ \\
\hline $\mathrm{Si}(1)-\mathrm{C}(18)$ & $1.842(13)$ \\
\hline $\mathrm{Si}(1)-\mathrm{C}(19)$ & $1.827(15)$ \\
\hline $\mathrm{Si}(1)-\mathrm{C}(20)$ & $1.831(15)$ \\
\hline $\mathrm{C}(1)-\mathrm{H}(1)$ & 1.0000 \\
\hline $\mathrm{C}(1)-\mathrm{C}(2)$ & $1.487(15)$ \\
\hline $\mathrm{C}(1)-\mathrm{C}(4)$ & $1.549(16)$ \\
\hline $\mathrm{C}(1)-\mathrm{C}(6)$ & $1.530(15)$ \\
\hline $\mathrm{C}(2)-\mathrm{C}(3)$ & $1.191(16)$ \\
\hline $\mathrm{C}(4)-\mathrm{H}(4 \mathrm{~A})$ & 0.9900 \\
\hline $\mathrm{C}(4)-\mathrm{H}(4 \mathrm{~B})$ & 0.9900 \\
\hline $\mathrm{C}(4)-\mathrm{C}(5)$ & $1.507(17)$ \\
\hline $\mathrm{C}(5)-\mathrm{H}(5 \mathrm{~A})$ & 0.9900 \\
\hline $\mathrm{C}(5)-\mathrm{H}(5 \mathrm{~B})$ & 0.9900 \\
\hline$C(6)-C(7)$ & $1.416(14)$ \\
\hline$C(6)-C(11)$ & $1.369(16)$ \\
\hline $\mathrm{C}(7)-\mathrm{H}(7)$ & 0.9500 \\
\hline $\mathrm{C}(7)-\mathrm{C}(8)$ & $1.386(17)$ \\
\hline $\mathrm{C}(8)-\mathrm{H}(8)$ & 0.9500 \\
\hline $\mathrm{C}(8)-\mathrm{C}(9)$ & $1.394(17)$ \\
\hline $\mathrm{C}(9)-\mathrm{C}(10)$ & $1.398(15)$ \\
\hline $\mathrm{C}(9)-\mathrm{C}(12)$ & $1.477(15)$ \\
\hline $\mathrm{C}(10)-\mathrm{H}(10)$ & 0.9500 \\
\hline $\mathrm{C}(10)-\mathrm{C}(11)$ & $1.382(17)$ \\
\hline $\mathrm{C}(11)-\mathrm{H}(11)$ & 0.9500 \\
\hline $\mathrm{C}(12)-\mathrm{C}(13)$ & $1.402(15)$ \\
\hline $\mathrm{C}(12)-\mathrm{C}(17)$ & $1.392(16)$ \\
\hline $\mathrm{C}(13)-\mathrm{H}(13)$ & 0.9500 \\
\hline $\mathrm{C}(13)-\mathrm{C}(14)$ & $1.394(17)$ \\
\hline $\mathrm{C}(14)-\mathrm{H}(14)$ & 0.9500 \\
\hline $\mathrm{C}(14)-\mathrm{C}(15)$ & $1.369(18)$ \\
\hline $\mathrm{C}(15)-\mathrm{H}(15)$ & 0.9500 \\
\hline$C(15)-C(16)$ & $1.382(18)$ \\
\hline $\mathrm{C}(16)-\mathrm{H}(16)$ & 0.9500 \\
\hline$C(16)-C(17)$ & $1.382(18)$ \\
\hline
\end{tabular}




\begin{tabular}{|c|c|}
\hline $\mathrm{C}(17)-\mathrm{H}(17)$ & 0.9500 \\
\hline $\mathrm{C}(18)-\mathrm{H}(18 \mathrm{~A})$ & 0.9800 \\
\hline $\mathrm{C}(18)-\mathrm{H}(18 \mathrm{~B})$ & 0.9800 \\
\hline $\mathrm{C}(18)-\mathrm{H}(18 \mathrm{C})$ & 0.9800 \\
\hline $\mathrm{C}(19)-\mathrm{H}(19 \mathrm{~A})$ & 0.9800 \\
\hline $\mathrm{C}(19)-\mathrm{H}(19 \mathrm{~B})$ & 0.9800 \\
\hline $\mathrm{C}(19)-\mathrm{H}(19 \mathrm{C})$ & 0.9800 \\
\hline $\mathrm{C}(20)-\mathrm{H}(20 \mathrm{~A})$ & 0.9800 \\
\hline $\mathrm{C}(20)-\mathrm{H}(20 \mathrm{~B})$ & 0.9800 \\
\hline $\mathrm{C}(20)-\mathrm{H}(20 \mathrm{C})$ & 0.9800 \\
\hline$C(3)-\operatorname{Si}(1)-C(18)$ & $110.6(5)$ \\
\hline $\mathrm{C}(19)-\mathrm{Si}(1)-\mathrm{C}(3)$ & 109.0(6) \\
\hline $\mathrm{C}(19)-\mathrm{Si}(1)-\mathrm{C}(18)$ & $108.3(8)$ \\
\hline $\mathrm{C}(19)-\mathrm{Si}(1)-\mathrm{C}(20)$ & 110.4(9) \\
\hline$C(20)-S i(1)-C(3)$ & 107.1(7) \\
\hline $\mathrm{C}(20)-\mathrm{Si}(1)-\mathrm{C}(18)$ & $111.5(8)$ \\
\hline $\mathrm{C}(2)-\mathrm{C}(1)-\mathrm{H}(1)$ & 107.9 \\
\hline$C(2)-C(1)-C(4)$ & 111.1(9) \\
\hline $\mathrm{C}(2)-\mathrm{C}(1)-\mathrm{C}(6)$ & $111.6(10)$ \\
\hline $\mathrm{C}(4)-\mathrm{C}(1)-\mathrm{H}(1)$ & 107.9 \\
\hline $\mathrm{C}(6)-\mathrm{C}(1)-\mathrm{H}(1)$ & 107.9 \\
\hline$C(6)-C(1)-C(4)$ & $110.4(9)$ \\
\hline $\mathrm{C}(3)-\mathrm{C}(2)-\mathrm{C}(1)$ & $179.1(13)$ \\
\hline $\mathrm{C}(2)-\mathrm{C}(3)-\mathrm{Si}(1)$ & $175.4(11)$ \\
\hline $\mathrm{C}(1)-\mathrm{C}(4)-\mathrm{H}(4 \mathrm{~A})$ & 108.5 \\
\hline $\mathrm{C}(1)-\mathrm{C}(4)-\mathrm{H}(4 \mathrm{~B})$ & 108.5 \\
\hline $\mathrm{H}(4 \mathrm{~A})-\mathrm{C}(4)-\mathrm{H}(4 \mathrm{~B})$ & 107.5 \\
\hline$C(5)-C(4)-C(1)$ & $115.3(10)$ \\
\hline $\mathrm{C}(5)-\mathrm{C}(4)-\mathrm{H}(4 \mathrm{~A})$ & 108.5 \\
\hline $\mathrm{C}(5)-\mathrm{C}(4)-\mathrm{H}(4 \mathrm{~B})$ & 108.5 \\
\hline $\mathrm{Br}(1)-\mathrm{C}(5)-\mathrm{H}(5 \mathrm{~A})$ & 109.2 \\
\hline $\mathrm{Br}(1)-\mathrm{C}(5)-\mathrm{H}(5 \mathrm{~B})$ & 109.2 \\
\hline $\mathrm{C}(4)-\mathrm{C}(5)-\mathrm{Br}(1)$ & $112.2(7)$ \\
\hline $\mathrm{C}(4)-\mathrm{C}(5)-\mathrm{H}(5 \mathrm{~A})$ & 109.2 \\
\hline $\mathrm{C}(4)-\mathrm{C}(5)-\mathrm{H}(5 \mathrm{~B})$ & 109.2 \\
\hline $\mathrm{H}(5 \mathrm{~A})-\mathrm{C}(5)-\mathrm{H}(5 \mathrm{~B})$ & 107.9 \\
\hline$C(7)-C(6)-C(1)$ & $117.6(11)$ \\
\hline
\end{tabular}




\begin{tabular}{|c|c|}
\hline $\mathrm{C}(11)-\mathrm{C}(6)-\mathrm{C}(1)$ & 123.6(9) \\
\hline$C(11)-C(6)-C(7)$ & $118.8(10)$ \\
\hline $\mathrm{C}(6)-\mathrm{C}(7)-\mathrm{H}(7)$ & 120.1 \\
\hline $\mathrm{C}(8)-\mathrm{C}(7)-\mathrm{C}(6)$ & $119.8(12)$ \\
\hline $\mathrm{C}(8)-\mathrm{C}(7)-\mathrm{H}(7)$ & 120.1 \\
\hline $\mathrm{C}(7)-\mathrm{C}(8)-\mathrm{H}(8)$ & 119.1 \\
\hline $\mathrm{C}(7)-\mathrm{C}(8)-\mathrm{C}(9)$ & $121.8(10)$ \\
\hline $\mathrm{C}(9)-\mathrm{C}(8)-\mathrm{H}(8)$ & 119.1 \\
\hline $\mathrm{C}(8)-\mathrm{C}(9)-\mathrm{C}(10)$ & $116.8(10)$ \\
\hline $\mathrm{C}(8)-\mathrm{C}(9)-\mathrm{C}(12)$ & $122.0(9)$ \\
\hline$C(10)-C(9)-C(12)$ & $121.1(11)$ \\
\hline $\mathrm{C}(9)-\mathrm{C}(10)-\mathrm{H}(10)$ & 118.9 \\
\hline $\mathrm{C}(11)-\mathrm{C}(10)-\mathrm{C}(9)$ & $122.1(11)$ \\
\hline $\mathrm{C}(11)-\mathrm{C}(10)-\mathrm{H}(10)$ & 118.9 \\
\hline$C(6)-C(11)-C(10)$ & $120.7(10)$ \\
\hline $\mathrm{C}(6)-\mathrm{C}(11)-\mathrm{H}(11)$ & 119.7 \\
\hline $\mathrm{C}(10)-\mathrm{C}(11)-\mathrm{H}(11)$ & 119.7 \\
\hline $\mathrm{C}(13)-\mathrm{C}(12)-\mathrm{C}(9)$ & $121.4(10)$ \\
\hline $\mathrm{C}(17)-\mathrm{C}(12)-\mathrm{C}(9)$ & $121.7(10)$ \\
\hline $\mathrm{C}(17)-\mathrm{C}(12)-\mathrm{C}(13)$ & $116.9(10)$ \\
\hline $\mathrm{C}(12)-\mathrm{C}(13)-\mathrm{H}(13)$ & 119.2 \\
\hline $\mathrm{C}(14)-\mathrm{C}(13)-\mathrm{C}(12)$ & $121.6(11)$ \\
\hline C(14)-C(13)-H(13) & 119.2 \\
\hline $\mathrm{C}(13)-\mathrm{C}(14)-\mathrm{H}(14)$ & 119.9 \\
\hline $\mathrm{C}(15)-\mathrm{C}(14)-\mathrm{C}(13)$ & $120.1(10)$ \\
\hline $\mathrm{C}(15)-\mathrm{C}(14)-\mathrm{H}(14)$ & 119.9 \\
\hline $\mathrm{C}(14)-\mathrm{C}(15)-\mathrm{H}(15)$ & 120.5 \\
\hline$C(14)-C(15)-C(16)$ & $118.9(11)$ \\
\hline $\mathrm{C}(16)-\mathrm{C}(15)-\mathrm{H}(15)$ & 120.5 \\
\hline $\mathrm{C}(15)-\mathrm{C}(16)-\mathrm{H}(16)$ & 119.3 \\
\hline$C(15)-C(16)-C(17)$ & $121.4(12)$ \\
\hline $\mathrm{C}(17)-\mathrm{C}(16)-\mathrm{H}(16)$ & 119.3 \\
\hline $\mathrm{C}(12)-\mathrm{C}(17)-\mathrm{H}(17)$ & 119.6 \\
\hline$C(16)-C(17)-C(12)$ & $120.9(11)$ \\
\hline $\mathrm{C}(16)-\mathrm{C}(17)-\mathrm{H}(17)$ & 119.6 \\
\hline $\mathrm{Si}(1)-\mathrm{C}(18)-\mathrm{H}(18 \mathrm{~A})$ & 109.5 \\
\hline $\mathrm{Si}(1)-\mathrm{C}(18)-\mathrm{H}(18 \mathrm{~B})$ & 109.5 \\
\hline $\mathrm{Si}(1)-\mathrm{C}(18)-\mathrm{H}(18 \mathrm{C})$ & 109.5 \\
\hline
\end{tabular}




$\begin{array}{ll}\text { H(18A)-C(18)-H(18B) } & 109.5 \\ \text { H(18A)-C(18)-H(18C) } & 109.5 \\ \text { H(18B)-C(18)-H(18C) } & 109.5 \\ \text { Si(1)-C(19)-H(19A) } & 109.5 \\ \text { Si(1)-C(19)-H(19B) } & 109.5 \\ \text { Si(1)-C(19)-H(19C) } & 109.5 \\ \text { H(19A)-C(19)-H(19B) } & 109.5 \\ \text { H(19A)-C(19)-H(19C) } & 109.5 \\ \text { H(19B)-C(19)-H(19C) } & 109.5 \\ \text { Si(1)-C(20)-H(20A) } & 109.5 \\ \text { Si(1)-C(20)-H(20B) } & 109.5 \\ \text { Si(1)-C(20)-H(20C) } & 109.5 \\ \text { H(20A)-C(20)-H(20B) } & 109.5 \\ \text { H(20A)-C(20)-H(20C) } & 109.5 \\ \text { H(20B)-C(20)-H(20C) } & 109.5\end{array}$

Symmetry transformations used to generate equivalent atoms:

Table 4. Anisotropic displacement parameters $\left(\AA^{2} \times 10^{3}\right)$ for mj19476_0m. The anisotropic displacement factor exponent takes the form: $-2 \pi^{2}\left[h^{2} a^{* 2} U^{11}+\ldots+2 h k a^{*} b^{*} U^{12}\right]$

\begin{tabular}{lcccccc}
\hline & $\mathrm{U}^{11}$ & $\mathrm{U}^{22}$ & $\mathrm{U}^{33}$ & $\mathrm{U}^{23}$ & $\mathrm{U}^{13}$ & $\mathrm{U}^{12}$ \\
\hline $\mathrm{Br}(1)$ & $54(1)$ & $62(1)$ & $86(1)$ & $-2(1)$ & $19(1)$ & $3(1)$ \\
$\mathrm{Si}(1)$ & $48(2)$ & $52(2)$ & $69(1)$ & $-1(1)$ & $11(1)$ & $2(1)$ \\
$\mathrm{C}(1)$ & $30(5)$ & $50(6)$ & $82(6)$ & $-4(5)$ & $12(4)$ & $3(4)$ \\
$\mathrm{C}(2)$ & $43(6)$ & $49(6)$ & $76(6)$ & $-4(5)$ & $18(4)$ & $1(5)$ \\
$\mathrm{C}(3)$ & $44(6)$ & $53(6)$ & $80(6)$ & $-1(5)$ & $13(5)$ & $1(5)$ \\
$\mathrm{C}(4)$ & $52(7)$ & $40(6)$ & $85(6)$ & $5(4)$ & $18(5)$ & $3(4)$ \\
$\mathrm{C}(5)$ & $54(7)$ & $56(6)$ & $83(6)$ & $6(5)$ & $11(5)$ & $11(6)$ \\
$\mathrm{C}(6)$ & $39(6)$ & $36(5)$ & $78(6)$ & $3(4)$ & $8(4)$ & $-1(4)$ \\
$\mathrm{C}(7)$ & $58(7)$ & $49(6)$ & $79(6)$ & $-2(5)$ & $5(5)$ & $-10(5)$ \\
$\mathrm{C}(8)$ & $52(7)$ & $55(6)$ & $72(5)$ & $-6(5)$ & $10(4)$ & $-6(5)$ \\
$\mathrm{C}(9)$ & $52(6)$ & $40(5)$ & $69(5)$ & $2(4)$ & $11(4)$ & $2(5)$ \\
$\mathrm{C}(10)$ & $56(7)$ & $41(5)$ & $80(6)$ & $1(4)$ & $10(5)$ & $-13(5)$ \\
$\mathrm{C}(11)$ & $56(7)$ & $52(6)$ & $72(5)$ & $0(5)$ & $5(5)$ & $-6(5)$ \\
$\mathrm{C}(12)$ & $48(6)$ & $36(5)$ & $73(5)$ & $3(4)$ & $13(4)$ & $-5(4)$ \\
$\mathrm{C}(13)$ & $58(7)$ & $36(6)$ & $87(6)$ & $3(4)$ & $16(5)$ & $-5(4)$ \\
& & & & & &
\end{tabular}




$\begin{array}{lcccccc}\mathrm{C}(14) & 76(8) & 48(8) & 75(5) & -2(4) & 22(5) & 0(5) \\ \mathrm{C}(15) & 72(9) & 57(7) & 77(6) & 8(5) & 21(5) & 8(6) \\ \mathrm{C}(16) & 56(8) & 66(8) & 89(7) & 17(6) & 9(6) & -8(6) \\ \mathrm{C}(17) & 59(8) & 58(7) & 78(6) & 8(5) & 8(5) & -11(6) \\ \mathrm{C}(18) & 47(7) & 88(9) & 105(8) & -33(8) & 19(5) & -3(7) \\ \mathrm{C}(19) & 66(10) & 54(8) & 137(12) & -19(7) & 13(8) & 4(7) \\ \mathrm{C}(20) & 109(14) & 130(16) & 80(7) & 17(9) & -5(8) & -30(12)\end{array}$

Table 5. Hydrogen coordinates ( $\left.\mathrm{x} 10^{4}\right)$ and isotropic displacement parameters $\left(\AA^{2} \times 10^{3}\right)$ for mj19476_0m.

\begin{tabular}{|c|c|c|c|c|}
\hline & $\mathrm{x}$ & $\mathrm{y}$ & z & $\mathrm{U}(\mathrm{eq})$ \\
\hline $\mathrm{H}(1)$ & 2787 & 4020 & 6988 & 64 \\
\hline $\mathrm{H}(4 \mathrm{~A})$ & 3192 & 1207 & 6952 & 70 \\
\hline $\mathrm{H}(4 \mathrm{~B})$ & 5632 & 1302 & 7363 & 70 \\
\hline $\mathrm{H}(5 \mathrm{~A})$ & 4346 & 2078 & 8449 & 77 \\
\hline $\mathrm{H}(5 \mathrm{~B})$ & 3385 & 349 & 8169 & 77 \\
\hline $\mathrm{H}(7)$ & 2848 & 2542 & 5761 & 75 \\
\hline $\mathrm{H}(8)$ & 4403 & 2575 & 4689 & 71 \\
\hline $\mathrm{H}(10)$ & 9651 & 4912 & 5738 & 70 \\
\hline $\mathrm{H}(11)$ & 8115 & 4904 & 6807 & 72 \\
\hline $\mathrm{H}(13)$ & 6164 & 2113 & 3842 & 72 \\
\hline $\mathrm{H}(14)$ & 7862 & 2010 & 2796 & 78 \\
\hline $\mathrm{H}(15)$ & 10913 & 3602 & 2713 & 81 \\
\hline $\mathrm{H}(16)$ & 12273 & 5269 & 3691 & 85 \\
\hline $\mathrm{H}(17)$ & 10541 & 5452 & 4719 & 78 \\
\hline $\mathrm{H}(18 \mathrm{~A})$ & 11350 & 5443 & 8754 & 119 \\
\hline $\mathrm{H}(18 \mathrm{~B})$ & 11687 & 6799 & 9380 & 119 \\
\hline $\mathrm{H}(18 \mathrm{C})$ & 11115 & 7325 & 8541 & 119 \\
\hline $\mathrm{H}(19 \mathrm{~A})$ & 6811 & 8982 & 8523 & 129 \\
\hline $\mathrm{H}(19 \mathrm{~B})$ & 7938 & 9141 & 9355 & 129 \\
\hline $\mathrm{H}(19 \mathrm{C})$ & 5491 & 8489 & 9174 & 129 \\
\hline $\mathrm{H}(20 \mathrm{~A})$ & 5898 & 5014 & 9757 & 162 \\
\hline $\mathrm{H}(20 \mathrm{~B})$ & 7846 & 5978 & 10233 & 162 \\
\hline $\mathrm{H}(20 \mathrm{C})$ & 8323 & 4306 & 9844 & 162 \\
\hline
\end{tabular}

Table 6. Torsion angles $\left[{ }^{\circ}\right]$ for mj19476_0m. 


\begin{tabular}{lc}
\hline $\mathrm{C}(2)-\mathrm{C}(1)-\mathrm{C}(4)-\mathrm{C}(5)$ & $49.8(14)$ \\
$\mathrm{C}(6)-\mathrm{C}(1)-\mathrm{C}(4)-\mathrm{C}(5)$ & $174.2(9)$ \\
$\mathrm{C}(1)-\mathrm{C}(4)-\mathrm{C}(5)-\mathrm{Br}(1)$ & $61.2(12)$ \\
$\mathrm{C}(2)-\mathrm{C}(1)-\mathrm{C}(6)-\mathrm{C}(11)$ & $10.9(15)$ \\
$\mathrm{C}(4)-\mathrm{C}(1)-\mathrm{C}(6)-\mathrm{C}(11)$ & $-113.1(11)$ \\
$\mathrm{C}(2)-\mathrm{C}(1)-\mathrm{C}(6)-\mathrm{C}(7)$ & $-170.9(10)$ \\
$\mathrm{C}(4)-\mathrm{C}(1)-\mathrm{C}(6)-\mathrm{C}(7)$ & $65.1(13)$ \\
$\mathrm{C}(11)-\mathrm{C}(6)-\mathrm{C}(7)-\mathrm{C}(8)$ & $0.8(17)$ \\
$\mathrm{C}(1)-\mathrm{C}(6)-\mathrm{C}(7)-\mathrm{C}(8)$ & $-177.4(10)$ \\
$\mathrm{C}(6)-\mathrm{C}(7)-\mathrm{C}(8)-\mathrm{C}(9)$ & $-0.2(18)$ \\
$\mathrm{C}(7)-\mathrm{C}(8)-\mathrm{C}(9)-\mathrm{C}(10)$ & $-0.1(18)$ \\
$\mathrm{C}(7)-\mathrm{C}(8)-\mathrm{C}(9)-\mathrm{C}(12)$ & $178.1(11)$ \\
$\mathrm{C}(8)-\mathrm{C}(9)-\mathrm{C}(10)-\mathrm{C}(11)$ & $-0.1(17)$ \\
$\mathrm{C}(12)-\mathrm{C}(9)-\mathrm{C}(10)-\mathrm{C}(11)$ & $-178.3(10)$ \\
$\mathrm{C}(7)-\mathrm{C}(6)-\mathrm{C}(11)-\mathrm{C}(10)$ & $-1.0(17)$ \\
$\mathrm{C}(1)-\mathrm{C}(6)-\mathrm{C}(11)-\mathrm{C}(10)$ & $177.1(10)$ \\
$\mathrm{C}(9)-\mathrm{C}(10)-\mathrm{C}(11)-\mathrm{C}(6)$ & $0.7(18)$ \\
$\mathrm{C}(8)-\mathrm{C}(9)-\mathrm{C}(12)-\mathrm{C}(17)$ & $165.9(11)$ \\
$\mathrm{C}(10)-\mathrm{C}(9)-\mathrm{C}(12)-\mathrm{C}(17)$ & $-15.9(17)$ \\
$\mathrm{C}(8)-\mathrm{C}(9)-\mathrm{C}(12)-\mathrm{C}(13)$ & $-12.3(17)$ \\
$\mathrm{C}(10)-\mathrm{C}(9)-\mathrm{C}(12)-\mathrm{C}(13)$ & $165.8(11)$ \\
$\mathrm{C}(17)-\mathrm{C}(12)-\mathrm{C}(13)-\mathrm{C}(14)$ & $3.5(16)$ \\
$\mathrm{C}(9)-\mathrm{C}(12)-\mathrm{C}(13)-\mathrm{C}(14)$ & $-178.1(10)$ \\
$\mathrm{C}(12)-\mathrm{C}(13)-\mathrm{C}(14)-\mathrm{C}(15)$ & $-1.2(17)$ \\
$\mathrm{C}(13)-\mathrm{C}(14)-\mathrm{C}(15)-\mathrm{C}(16)$ & $-0.1(18)$ \\
$\mathrm{C}(14)-\mathrm{C}(15)-\mathrm{C}(16)-\mathrm{C}(17)$ & $-1.0(19)$ \\
$\mathrm{C}(15)-\mathrm{C}(16)-\mathrm{C}(17)-\mathrm{C}(12)$ & $3.5(19)$ \\
$\mathrm{C}(13)-\mathrm{C}(12)-\mathrm{C}(17)-\mathrm{C}(16)$ & $-4.6(17)$ \\
$\mathrm{C}(9)-\mathrm{C}(12)-\mathrm{C}(17)-\mathrm{C}(16)$ & \\
\hline & \\
\hline & \\
& \\
&
\end{tabular}


<smiles>CC(C#CC(C)(C)C)c1cccc2sc(-c3ccccc3)nc12</smiles>

4d
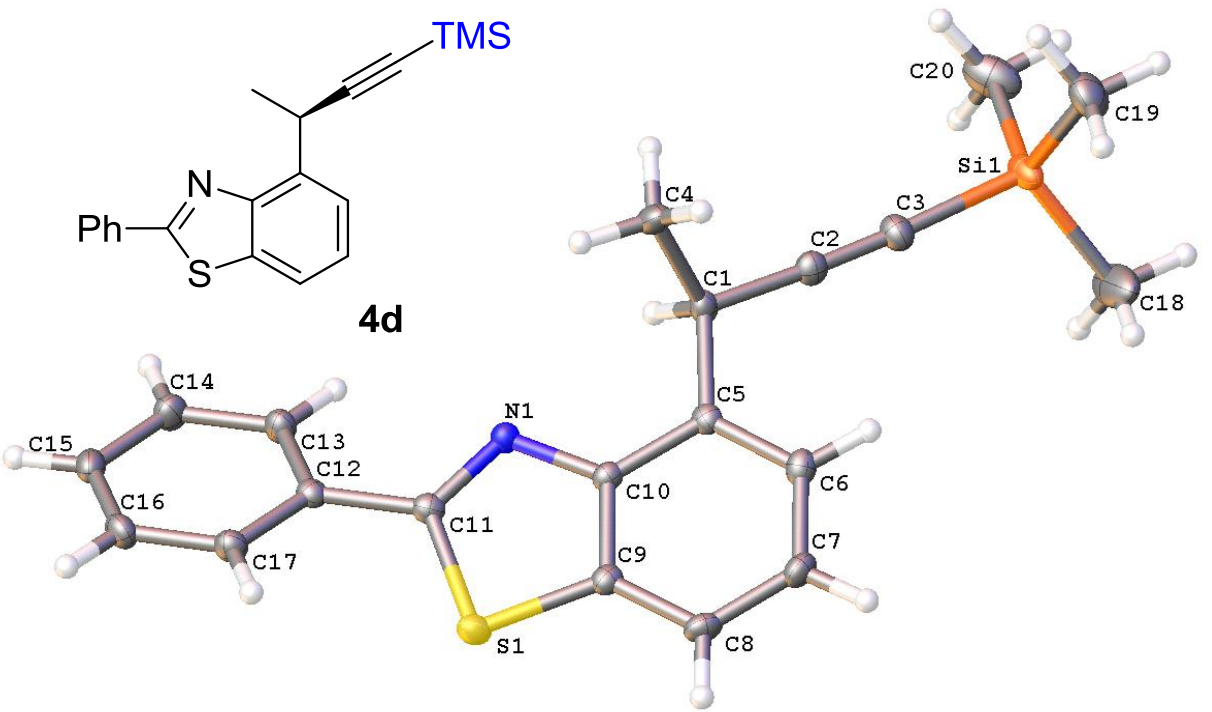

Table 1. Crystal data and structure refinement for mj19475.

Identification code

Empirical formula

Formula weight

Temperature

Wavelength

Crystal system

Space group

Unit cell dimensions

Volume

$\mathrm{Z}$

Density (calculated)

Absorption coefficient

$\mathrm{F}(000)$

Crystal size

Theta range for data collection

Index ranges

Reflections collected

Independent reflections

Completeness to theta $=53.594^{\circ}$

Absorption correction

Max. and min. transmission

Refinement method

Data / restraints / parameters mj19475

C20 H21 N S Si

335.53

$169.97 \mathrm{~K}$

$1.34139 \AA$

Orthorhombic

$\mathrm{P} 2{ }_{1} 2_{1} 2_{1}$

$\mathrm{a}=9.5614(2) \AA$ $\alpha=90^{\circ}$.

$\mathrm{b}=26.9394(5) \AA$

$\beta=90^{\circ}$.

$\mathrm{c}=7.32500(10) \AA$ $\gamma=90^{\circ}$.
4

$1.181 \mathrm{Mg} / \mathrm{m}^{3}$

$1.367 \mathrm{~mm}^{-1}$

712

$0.12 \times 0.1 \times 0.08 \mathrm{~mm}^{3}$

4.269 to $54.961^{\circ}$.

$-11<=\mathrm{h}<=11,-32<=\mathrm{k}<=32,-8<=1<=7$

20486

$3571[\mathrm{R}(\mathrm{int})=0.0626]$

$99.9 \%$

Semi-empirical from equivalents

0.7508 and 0.5859

Full-matrix least-squares on $\mathrm{F}^{2}$

$3571 / 0 / 212$ 
Goodness-of-fit on $\mathrm{F}^{2}$

Final R indices [I $>2 \operatorname{sigma}(\mathrm{I})]$

$\mathrm{R}$ indices (all data)

Absolute structure parameter

Extinction coefficient

Largest diff. peak and hole
1.093

$$
\begin{aligned}
& \mathrm{R} 1=0.0391, \mathrm{wR} 2=0.0796 \\
& \mathrm{R} 1=0.0472, \mathrm{wR} 2=0.0831 \\
& 0.036(13) \\
& \mathrm{n} / \mathrm{a} \\
& 0.228 \text { and }-0.291 \mathrm{e} . \AA^{-3}
\end{aligned}
$$

\begin{tabular}{|c|c|c|c|c|}
\hline & $\mathrm{x}$ & $\mathrm{y}$ & $\mathrm{z}$ & $\mathrm{U}(\mathrm{eq})$ \\
\hline$S(1)$ & 4929(1) & $4824(1)$ & $2949(1)$ & $30(1)$ \\
\hline $\operatorname{Si}(1)$ & $-666(1)$ & 7191(1) & $8333(2)$ & $39(1)$ \\
\hline $\mathrm{N}(1)$ & $4586(2)$ & $5299(1)$ & $6007(3)$ & $25(1)$ \\
\hline $\mathrm{C}(1)$ & $2875(3)$ & $6160(1)$ & $6963(5)$ & $27(1)$ \\
\hline $\mathrm{C}(2)$ & $1673(3)$ & $6486(1)$ & $7342(4)$ & $29(1)$ \\
\hline $\mathrm{C}(3)$ & $730(3)$ & $6760(1)$ & $7702(5)$ & $35(1)$ \\
\hline $\mathrm{C}(4)$ & $4243(3)$ & $6441(1)$ & $7364(5)$ & $38(1)$ \\
\hline$C(5)$ & $2888(3)$ & $5943(1)$ & $5056(4)$ & $24(1)$ \\
\hline $\mathrm{C}(6)$ & $2127(3)$ & $6131(1)$ & $3626(5)$ & $30(1)$ \\
\hline $\mathrm{C}(7)$ & $2202(3)$ & $5929(1)$ & $1864(5)$ & $32(1)$ \\
\hline $\mathrm{C}(8)$ & $3035(3)$ & $5522(1)$ & $1503(5)$ & $31(1)$ \\
\hline $\mathrm{C}(9)$ & $3801(3)$ & $5325(1)$ & $2947(5)$ & $26(1)$ \\
\hline $\mathrm{C}(10)$ & $3740(3)$ & $5529(1)$ & $4709(4)$ & $23(1)$ \\
\hline $\mathrm{C}(11)$ & $5270(3)$ & 4931(1) & $5274(4)$ & $25(1)$ \\
\hline $\mathrm{C}(12)$ & $6244(3)$ & $4610(1)$ & $6271(5)$ & $25(1)$ \\
\hline $\mathrm{C}(13)$ & $6101(3)$ & $4551(1)$ & $8138(5)$ & $31(1)$ \\
\hline $\mathrm{C}(14)$ & $6997(4)$ & $4244(1)$ & $9095(5)$ & $37(1)$ \\
\hline$C(15)$ & $8053(3)$ & 3991(1) & $8176(5)$ & $34(1)$ \\
\hline$C(16)$ & $8210(3)$ & $4053(1)$ & $6331(5)$ & $34(1)$ \\
\hline $\mathrm{C}(17)$ & $7313(3)$ & $4357(1)$ & $5364(5)$ & $31(1)$ \\
\hline $\mathrm{C}(18)$ & $-2278(4)$ & $7004(2)$ & $7106(8)$ & $88(2)$ \\
\hline $\mathrm{C}(19)$ & $-110(4)$ & $7823(1)$ & $7653(6)$ & $56(1)$ \\
\hline $\mathrm{C}(20)$ & $-916(5)$ & $7154(2)$ & 10849(6) & $64(1)$ \\
\hline
\end{tabular}

Table 2. Atomic coordinates ( $\left.\mathrm{x} 10^{4}\right)$ and equivalent isotropic displacement parameters $\left(\AA^{2} \mathrm{x} 10^{3}\right)$ for $\mathrm{mj} 19475$. $U(\mathrm{eq})$ is defined as one third of the trace of the orthogonalized $\mathrm{U}^{\mathrm{ij}}$ tensor.

Table 3. Bond lengths $[\AA]$ and angles $\left[{ }^{\circ}\right]$ for mj19475. 


\begin{tabular}{|c|c|}
\hline$S(1)-C(11)$ & $1.758(3)$ \\
\hline $\operatorname{Si}(1)-C(3)$ & $1.830(3)$ \\
\hline $\mathrm{Si}(1)-\mathrm{C}(18)$ & $1.854(4)$ \\
\hline $\mathrm{Si}(1)-\mathrm{C}(19)$ & $1.852(4)$ \\
\hline $\mathrm{Si}(1)-\mathrm{C}(20)$ & $1.862(4)$ \\
\hline $\mathrm{N}(1)-\mathrm{C}(10)$ & $1.394(4)$ \\
\hline $\mathrm{N}(1)-\mathrm{C}(11)$ & $1.303(4)$ \\
\hline $\mathrm{C}(1)-\mathrm{H}(1)$ & 1.0000 \\
\hline$C(1)-C(2)$ & $1.473(4)$ \\
\hline$C(1)-C(4)$ & $1.540(4)$ \\
\hline$C(1)-C(5)$ & $1.515(4)$ \\
\hline$C(2)-C(3)$ & $1.194(4)$ \\
\hline $\mathrm{C}(4)-\mathrm{H}(4 \mathrm{~A})$ & 0.9800 \\
\hline $\mathrm{C}(4)-\mathrm{H}(4 \mathrm{~B})$ & 0.9800 \\
\hline $\mathrm{C}(4)-\mathrm{H}(4 \mathrm{C})$ & 0.9800 \\
\hline$C(5)-C(6)$ & $1.373(4)$ \\
\hline$C(5)-C(10)$ & $1.403(4)$ \\
\hline $\mathrm{C}(6)-\mathrm{H}(6)$ & 0.9500 \\
\hline$C(6)-C(7)$ & $1.403(5)$ \\
\hline $\mathrm{C}(7)-\mathrm{H}(7)$ & 0.9500 \\
\hline $\mathrm{C}(7)-\mathrm{C}(8)$ & $1.379(4)$ \\
\hline $\mathrm{C}(8)-\mathrm{H}(8)$ & 0.9500 \\
\hline $\mathrm{C}(8)-\mathrm{C}(9)$ & $1.392(4)$ \\
\hline $\mathrm{C}(9)-\mathrm{C}(10)$ & $1.404(4)$ \\
\hline$C(11)-C(12)$ & $1.466(4)$ \\
\hline$C(12)-C(13)$ & $1.384(5)$ \\
\hline$C(12)-C(17)$ & $1.396(4)$ \\
\hline $\mathrm{C}(13)-\mathrm{H}(13)$ & 0.9500 \\
\hline$C(13)-C(14)$ & $1.382(4)$ \\
\hline $\mathrm{C}(14)-\mathrm{H}(14)$ & 0.9500 \\
\hline $\mathrm{C}(14)-\mathrm{C}(15)$ & $1.392(5)$ \\
\hline $\mathrm{C}(15)-\mathrm{H}(15)$ & 0.9500 \\
\hline$C(15)-C(16)$ & $1.370(5)$ \\
\hline $\mathrm{C}(16)-\mathrm{H}(16)$ & 0.9500 \\
\hline$C(16)-C(17)$ & $1.381(4)$ \\
\hline $\mathrm{C}(17)-\mathrm{H}(17)$ & 0.9500 \\
\hline $\mathrm{C}(18)-\mathrm{H}(18 \mathrm{~A})$ & 0.9800 \\
\hline $\mathrm{C}(18)-\mathrm{H}(18 \mathrm{~B})$ & 0.9800 \\
\hline
\end{tabular}




\begin{tabular}{|c|c|}
\hline $\mathrm{C}(18)-\mathrm{H}(18 \mathrm{C})$ & 0.9800 \\
\hline $\mathrm{C}(19)-\mathrm{H}(19 \mathrm{~A})$ & 0.9800 \\
\hline C(19)-H(19B) & 0.9800 \\
\hline $\mathrm{C}(19)-\mathrm{H}(19 \mathrm{C})$ & 0.9800 \\
\hline $\mathrm{C}(20)-\mathrm{H}(20 \mathrm{~A})$ & 0.9800 \\
\hline $\mathrm{C}(20)-\mathrm{H}(20 \mathrm{~B})$ & 0.9800 \\
\hline $\mathrm{C}(20)-\mathrm{H}(20 \mathrm{C})$ & 0.9800 \\
\hline $\mathrm{C}(9)-\mathrm{S}(1)-\mathrm{C}(11)$ & $89.28(15)$ \\
\hline $\mathrm{C}(3)-\mathrm{Si}(1)-\mathrm{C}(18)$ & $108.13(18)$ \\
\hline $\mathrm{C}(3)-\mathrm{Si}(1)-\mathrm{C}(19)$ & $107.86(17)$ \\
\hline $\mathrm{C}(3)-\mathrm{Si}(1)-\mathrm{C}(20)$ & 107.99(19) \\
\hline $\mathrm{C}(18)-\mathrm{Si}(1)-\mathrm{C}(20)$ & $111.0(2)$ \\
\hline$C(19)-\operatorname{Si}(1)-C(18)$ & $111.0(2)$ \\
\hline$C(19)-\operatorname{Si}(1)-C(20)$ & $110.68(19)$ \\
\hline $\mathrm{C}(11)-\mathrm{N}(1)-\mathrm{C}(10)$ & $110.4(3)$ \\
\hline $\mathrm{C}(2)-\mathrm{C}(1)-\mathrm{H}(1)$ & 107.2 \\
\hline$C(2)-C(1)-C(4)$ & $109.5(2)$ \\
\hline$C(2)-C(1)-C(5)$ & $114.3(3)$ \\
\hline $\mathrm{C}(4)-\mathrm{C}(1)-\mathrm{H}(1)$ & 107.2 \\
\hline $\mathrm{C}(5)-\mathrm{C}(1)-\mathrm{H}(1)$ & 107.2 \\
\hline $\mathrm{C}(5)-\mathrm{C}(1)-\mathrm{C}(4)$ & $111.0(3)$ \\
\hline$C(3)-C(2)-C(1)$ & $177.4(4)$ \\
\hline $\mathrm{C}(2)-\mathrm{C}(3)-\mathrm{Si}(1)$ & $177.5(3)$ \\
\hline $\mathrm{C}(1)-\mathrm{C}(4)-\mathrm{H}(4 \mathrm{~A})$ & 109.5 \\
\hline $\mathrm{C}(1)-\mathrm{C}(4)-\mathrm{H}(4 \mathrm{~B})$ & 109.5 \\
\hline $\mathrm{C}(1)-\mathrm{C}(4)-\mathrm{H}(4 \mathrm{C})$ & 109.5 \\
\hline $\mathrm{H}(4 \mathrm{~A})-\mathrm{C}(4)-\mathrm{H}(4 \mathrm{~B})$ & 109.5 \\
\hline $\mathrm{H}(4 \mathrm{~A})-\mathrm{C}(4)-\mathrm{H}(4 \mathrm{C})$ & 109.5 \\
\hline $\mathrm{H}(4 \mathrm{~B})-\mathrm{C}(4)-\mathrm{H}(4 \mathrm{C})$ & 109.5 \\
\hline$C(6)-C(5)-C(1)$ & $123.8(3)$ \\
\hline$C(6)-C(5)-C(10)$ & $117.6(3)$ \\
\hline $\mathrm{C}(10)-\mathrm{C}(5)-\mathrm{C}(1)$ & $118.6(3)$ \\
\hline $\mathrm{C}(5)-\mathrm{C}(6)-\mathrm{H}(6)$ & 119.0 \\
\hline$C(5)-C(6)-C(7)$ & 122.1(3) \\
\hline $\mathrm{C}(7)-\mathrm{C}(6)-\mathrm{H}(6)$ & 119.0 \\
\hline $\mathrm{C}(6)-\mathrm{C}(7)-\mathrm{H}(7)$ & 119.5 \\
\hline $\mathrm{C}(8)-\mathrm{C}(7)-\mathrm{C}(6)$ & $120.9(3)$ \\
\hline
\end{tabular}




\begin{tabular}{|c|c|}
\hline $\mathrm{C}(8)-\mathrm{C}(7)-\mathrm{H}(7)$ & 119.5 \\
\hline $\mathrm{C}(7)-\mathrm{C}(8)-\mathrm{H}(8)$ & 121.2 \\
\hline$C(7)-C(8)-C(9)$ & $117.5(3)$ \\
\hline $\mathrm{C}(9)-\mathrm{C}(8)-\mathrm{H}(8)$ & 121.2 \\
\hline $\mathrm{C}(8)-\mathrm{C}(9)-\mathrm{S}(1)$ & $128.9(3)$ \\
\hline$C(8)-C(9)-C(10)$ & $121.8(3)$ \\
\hline $\mathrm{C}(10)-\mathrm{C}(9)-\mathrm{S}(1)$ & $109.4(2)$ \\
\hline $\mathrm{N}(1)-\mathrm{C}(10)-\mathrm{C}(5)$ & $124.5(3)$ \\
\hline $\mathrm{N}(1)-\mathrm{C}(10)-\mathrm{C}(9)$ & $115.4(3)$ \\
\hline$C(5)-C(10)-C(9)$ & $120.1(3)$ \\
\hline $\mathrm{N}(1)-\mathrm{C}(11)-\mathrm{S}(1)$ & $115.6(2)$ \\
\hline $\mathrm{N}(1)-\mathrm{C}(11)-\mathrm{C}(12)$ & $124.2(3)$ \\
\hline$C(12)-C(11)-S(1)$ & $120.2(2)$ \\
\hline$C(13)-C(12)-C(11)$ & $119.8(3)$ \\
\hline$C(13)-C(12)-C(17)$ & 119.1(3) \\
\hline$C(17)-C(12)-C(11)$ & $121.1(3)$ \\
\hline $\mathrm{C}(12)-\mathrm{C}(13)-\mathrm{H}(13)$ & 119.7 \\
\hline$C(14)-C(13)-C(12)$ & $120.6(3)$ \\
\hline $\mathrm{C}(14)-\mathrm{C}(13)-\mathrm{H}(13)$ & 119.7 \\
\hline $\mathrm{C}(13)-\mathrm{C}(14)-\mathrm{H}(14)$ & 120.1 \\
\hline$C(13)-C(14)-C(15)$ & $119.8(3)$ \\
\hline $\mathrm{C}(15)-\mathrm{C}(14)-\mathrm{H}(14)$ & 120.1 \\
\hline $\mathrm{C}(14)-\mathrm{C}(15)-\mathrm{H}(15)$ & 120.1 \\
\hline$C(16)-C(15)-C(14)$ & $119.8(3)$ \\
\hline$C(16)-C(15)-H(15)$ & 120.1 \\
\hline $\mathrm{C}(15)-\mathrm{C}(16)-\mathrm{H}(16)$ & 119.7 \\
\hline$C(15)-C(16)-C(17)$ & $120.7(3)$ \\
\hline $\mathrm{C}(17)-\mathrm{C}(16)-\mathrm{H}(16)$ & 119.7 \\
\hline $\mathrm{C}(12)-\mathrm{C}(17)-\mathrm{H}(17)$ & 120.0 \\
\hline$C(16)-C(17)-C(12)$ & $120.0(3)$ \\
\hline $\mathrm{C}(16)-\mathrm{C}(17)-\mathrm{H}(17)$ & 120.0 \\
\hline $\mathrm{Si}(1)-\mathrm{C}(18)-\mathrm{H}(18 \mathrm{~A})$ & 109.5 \\
\hline $\mathrm{Si}(1)-\mathrm{C}(18)-\mathrm{H}(18 \mathrm{~B})$ & 109.5 \\
\hline $\mathrm{Si}(1)-\mathrm{C}(18)-\mathrm{H}(18 \mathrm{C})$ & 109.5 \\
\hline $\mathrm{H}(18 \mathrm{~A})-\mathrm{C}(18)-\mathrm{H}(18 \mathrm{~B})$ & 109.5 \\
\hline $\mathrm{H}(18 \mathrm{~A})-\mathrm{C}(18)-\mathrm{H}(18 \mathrm{C})$ & 109.5 \\
\hline $\mathrm{H}(18 \mathrm{~B})-\mathrm{C}(18)-\mathrm{H}(18 \mathrm{C})$ & 109.5 \\
\hline $\mathrm{Si}(1)-\mathrm{C}(19)-\mathrm{H}(19 \mathrm{~A})$ & 109.5 \\
\hline
\end{tabular}




$\begin{array}{ll}\mathrm{Si}(1)-\mathrm{C}(19)-\mathrm{H}(19 B) & 109.5 \\ \text { Si(1)-C(19)-H(19C) } & 109.5 \\ \text { H(19A)-C(19)-H(19B) } & 109.5 \\ \text { H(19A)-C(19)-H(19C) } & 109.5 \\ \text { H(19B)-C(19)-H(19C) } & 109.5 \\ \text { Si(1)-C(20)-H(20A) } & 109.5 \\ \text { Si(1)-C(20)-H(20B) } & 109.5 \\ \text { Si(1)-C(20)-H(20C) } & 109.5 \\ \text { H(20A)-C(20)-H(20B) } & 109.5 \\ \text { H(20A)-C(20)-H(20C) } & 109.5 \\ \text { H(20B)-C(20)-H(20C) } & 109.5\end{array}$

Symmetry transformations used to generate equivalent atoms:

Table 4. Anisotropic displacement parameters $\left(\AA^{2} \times 10^{3}\right)$ for mj19475. The anisotropic displacement factor exponent takes the form: $-2 \pi^{2}\left[h^{2} a^{* 2} U^{11}+\ldots+2 h k a^{*} b^{*} U^{12}\right]$

\begin{tabular}{lcccccc}
\hline & $\mathrm{U}^{11}$ & $\mathrm{U}^{22}$ & $\mathrm{U}^{33}$ & $\mathrm{U}^{23}$ & $\mathrm{U}^{13}$ & $\mathrm{U}^{12}$ \\
\hline $\mathrm{S}(1)$ & $38(1)$ & $28(1)$ & $25(1)$ & $-3(1)$ & $0(1)$ & $2(1)$ \\
$\mathrm{Si}(1)$ & $25(1)$ & $33(1)$ & $59(1)$ & $-14(1)$ & $3(1)$ & $2(1)$ \\
$\mathrm{N}(1)$ & $26(1)$ & $26(1)$ & $22(1)$ & $1(1)$ & $0(1)$ & $2(1)$ \\
$\mathrm{C}(1)$ & $30(2)$ & $27(2)$ & $24(2)$ & $3(1)$ & $-1(2)$ & $6(1)$ \\
$\mathrm{C}(2)$ & $32(2)$ & $31(2)$ & $25(2)$ & $4(2)$ & $1(1)$ & $1(1)$ \\
$\mathrm{C}(3)$ & $33(2)$ & $31(2)$ & $40(2)$ & $-1(2)$ & $3(2)$ & $2(2)$ \\
$\mathrm{C}(4)$ & $32(2)$ & $46(2)$ & $35(2)$ & $-14(2)$ & $-8(2)$ & $5(2)$ \\
$\mathrm{C}(5)$ & $20(2)$ & $25(2)$ & $27(2)$ & $1(1)$ & $2(1)$ & $-2(1)$ \\
$\mathrm{C}(6)$ & $26(2)$ & $31(2)$ & $32(2)$ & $7(2)$ & $1(2)$ & $0(1)$ \\
$\mathrm{C}(7)$ & $33(2)$ & $36(2)$ & $28(2)$ & $5(2)$ & $-9(2)$ & $-1(1)$ \\
$\mathrm{C}(8)$ & $39(2)$ & $35(2)$ & $20(2)$ & $-1(1)$ & $-3(2)$ & $-7(2)$ \\
$\mathrm{C}(9)$ & $26(1)$ & $25(2)$ & $26(2)$ & $3(2)$ & $-2(1)$ & $-4(1)$ \\
$\mathrm{C}(10)$ & $24(1)$ & $25(2)$ & $20(2)$ & $2(1)$ & $0(1)$ & $-3(1)$ \\
$\mathrm{C}(11)$ & $27(2)$ & $25(2)$ & $23(2)$ & $1(1)$ & $3(1)$ & $-3(1)$ \\
$\mathrm{C}(12)$ & $26(2)$ & $21(2)$ & $28(2)$ & $-1(1)$ & $1(1)$ & $-2(1)$ \\
$\mathrm{C}(13)$ & $33(2)$ & $34(2)$ & $27(2)$ & $-2(2)$ & $1(2)$ & $7(1)$ \\
$\mathrm{C}(14)$ & $44(2)$ & $39(2)$ & $29(2)$ & $1(2)$ & $-5(2)$ & $7(2)$ \\
$\mathrm{C}(15)$ & $33(2)$ & $28(2)$ & $43(2)$ & $-1(2)$ & $-10(2)$ & $4(1)$ \\
$\mathrm{C}(16)$ & $32(2)$ & $30(2)$ & $41(2)$ & $-6(2)$ & $2(2)$ & $7(1)$ \\
& & & & & &
\end{tabular}




$\begin{array}{lllcccc}\mathrm{C}(17) & 34(2) & 31(2) & 27(2) & -2(2) & 2(2) & 3(1) \\ \mathrm{C}(18) & 36(2) & 89(3) & 138(5) & -57(4) & -23(3) & 14(2) \\ \mathrm{C}(19) & 63(2) & 36(2) & 69(3) & 2(2) & -1(2) & 9(2) \\ \mathrm{C}(20) & 70(3) & 55(3) & 65(3) & -14(2) & 31(2) & -11(2)\end{array}$

Table 5. Hydrogen coordinates ( $\left.\times 10^{4}\right)$ and isotropic displacement parameters $\left(\AA^{2} \times 10^{3}\right)$ for mj19475.

\begin{tabular}{|c|c|c|c|c|}
\hline & $\mathrm{x}$ & $\mathrm{y}$ & z & $\mathrm{U}(\mathrm{eq})$ \\
\hline $\mathrm{H}(1)$ & 2822 & 5876 & 7839 & 32 \\
\hline $\mathrm{H}(4 \mathrm{~A})$ & 5039 & 6216 & 7201 & 56 \\
\hline $\mathrm{H}(4 \mathrm{~B})$ & 4229 & 6564 & 8623 & 56 \\
\hline $\mathrm{H}(4 \mathrm{C})$ & 4332 & 6722 & 6522 & 56 \\
\hline $\mathrm{H}(6)$ & 1532 & 6408 & 3835 & 35 \\
\hline $\mathrm{H}(7)$ & 1671 & 6073 & 905 & 39 \\
\hline $\mathrm{H}(8)$ & 3085 & 5383 & 314 & 38 \\
\hline $\mathrm{H}(13)$ & 5381 & 4724 & 8768 & 37 \\
\hline $\mathrm{H}(14)$ & 6893 & 4206 & 10377 & 45 \\
\hline $\mathrm{H}(15)$ & 8663 & 3776 & 8826 & 41 \\
\hline $\mathrm{H}(16)$ & 8943 & 3885 & 5710 & 41 \\
\hline $\mathrm{H}(17)$ & 7423 & 4394 & 4082 & 37 \\
\hline $\mathrm{H}(18 \mathrm{~A})$ & -2121 & 7027 & 5787 & 132 \\
\hline $\mathrm{H}(18 \mathrm{~B})$ & -3048 & 7224 & 7454 & 132 \\
\hline $\mathrm{H}(18 \mathrm{C})$ & -2516 & 6661 & 7429 & 132 \\
\hline $\mathrm{H}(19 \mathrm{~A})$ & 750 & 7912 & 8305 & 83 \\
\hline $\mathrm{H}(19 \mathrm{~B})$ & -848 & 8061 & 7960 & 83 \\
\hline $\mathrm{H}(19 \mathrm{C})$ & 64 & 7831 & 6335 & 83 \\
\hline $\mathrm{H}(20 \mathrm{~A})$ & -1209 & 6817 & 11182 & 95 \\
\hline $\mathrm{H}(20 \mathrm{~B})$ & -1637 & 7391 & 11226 & 95 \\
\hline $\mathrm{H}(20 \mathrm{C})$ & -34 & 7233 & 11465 & 95 \\
\hline \multicolumn{5}{|c|}{ Table 6. Torsion angles $\left[^{\circ}\right]$ for mj 19475.} \\
\hline $\mathrm{C}(2)-\mathrm{C}(1)-\mathrm{C}(5)-\mathrm{C}(6)$ & & & $18.4(4)$ & \\
\hline $\mathrm{C}(4)-\mathrm{C}(1)-\mathrm{C}(5)-\mathrm{C}(6)$ & & & $-106.0(3)$ & \\
\hline$C(2)-C(1)-C(5)-C(10)$ & & & $-162.7(3)$ & \\
\hline$C(4)-C(1)-C(5)-C(10)$ & & & $72.9(3)$ & \\
\hline
\end{tabular}




$\begin{array}{lc}\mathrm{C}(10)-\mathrm{C}(5)-\mathrm{C}(6)-\mathrm{C}(7) & -1.0(4) \\ \mathrm{C}(1)-\mathrm{C}(5)-\mathrm{C}(6)-\mathrm{C}(7) & 177.9(3) \\ \mathrm{C}(5)-\mathrm{C}(6)-\mathrm{C}(7)-\mathrm{C}(8) & 1.0(5) \\ \mathrm{C}(6)-\mathrm{C}(7)-\mathrm{C}(8)-\mathrm{C}(9) & -0.4(4) \\ \mathrm{C}(7)-\mathrm{C}(8)-\mathrm{C}(9)-\mathrm{C}(10) & -0.3(4) \\ \mathrm{C}(7)-\mathrm{C}(8)-\mathrm{C}(9)-\mathrm{S}(1) & 180.0(2) \\ \mathrm{C}(11)-\mathrm{S}(1)-\mathrm{C}(9)-\mathrm{C}(8) & 178.5(3) \\ \mathrm{C}(11)-\mathrm{S}(1)-\mathrm{C}(9)-\mathrm{C}(10) & -1.3(2) \\ \mathrm{C}(11)-\mathrm{N}(1)-\mathrm{C}(10)-\mathrm{C}(5) & -179.0(3) \\ \mathrm{C}(11)-\mathrm{N}(1)-\mathrm{C}(10)-\mathrm{C}(9) & -0.1(4) \\ \mathrm{C}(6)-\mathrm{C}(5)-\mathrm{C}(10)-\mathrm{N}(1) & 179.2(3) \\ \mathrm{C}(1)-\mathrm{C}(5)-\mathrm{C}(10)-\mathrm{N}(1) & 0.2(4) \\ \mathrm{C}(6)-\mathrm{C}(5)-\mathrm{C}(10)-\mathrm{C}(9) & 0.4(4) \\ \mathrm{C}(1)-\mathrm{C}(5)-\mathrm{C}(10)-\mathrm{C}(9) & -178.6(3) \\ \mathrm{C}(8)-\mathrm{C}(9)-\mathrm{C}(10)-\mathrm{N}(1) & -178.7(3) \\ \mathrm{S}(1)-\mathrm{C}(9)-\mathrm{C}(10)-\mathrm{N}(1) & 1.1(3) \\ \mathrm{C}(8)-\mathrm{C}(9)-\mathrm{C}(10)-\mathrm{C}(5) & 0.3(4) \\ \mathrm{S}(1)-\mathrm{C}(9)-\mathrm{C}(10)-\mathrm{C}(5) & -179.9(2) \\ \mathrm{C}(10)-\mathrm{N}(1)-\mathrm{C}(11)-\mathrm{C}(12) & -179.6(3) \\ \mathrm{C}(10)-\mathrm{N}(1)-\mathrm{C}(11)-\mathrm{S}(1) & -1.0(3) \\ \mathrm{C}(9)-\mathrm{S}(1)-\mathrm{C}(11)-\mathrm{N}(1) & 1.4(2) \\ \mathrm{C}(9)-\mathrm{S}(1)-\mathrm{C}(11)-\mathrm{C}(12) & -179.9(2) \\ \mathrm{N}(1)-\mathrm{C}(11)-\mathrm{C}(12)-\mathrm{C}(13) & 25.2(4) \\ \mathrm{S}(1)-\mathrm{C}(11)-\mathrm{C}(12)-\mathrm{C}(13) & -153.4(2) \\ \mathrm{N}(1)-\mathrm{C}(11)-\mathrm{C}(12)-\mathrm{C}(17) & -155.5(3) \\ \mathrm{S}(1)-\mathrm{C}(11)-\mathrm{C}(12)-\mathrm{C}(17) & 26.0(4) \\ \mathrm{C}(17)-\mathrm{C}(12)-\mathrm{C}(13)-\mathrm{C}(14) & -0.4(5) \\ \mathrm{C}(11)-\mathrm{C}(12)-\mathrm{C}(13)-\mathrm{C}(14) & 179.0(3) \\ \mathrm{C}(12)-\mathrm{C}(13)-\mathrm{C}(14)-\mathrm{C}(15) & 0.0(5) \\ \mathrm{C}(13)-\mathrm{C}(14)-\mathrm{C}(15)-\mathrm{C}(16) & 0.8(5) \\ \mathrm{C}(14)-\mathrm{C}(15)-\mathrm{C}(16)-\mathrm{C}(17) & -1.3(5) \\ \mathrm{C}(15)-\mathrm{C}(16)-\mathrm{C}(17)-\mathrm{C}(12) & 0.8(5) \\ \mathrm{C}(13)-\mathrm{C}(12)-\mathrm{C}(17)-\mathrm{C}(16) & 0.0(5) \\ \mathrm{C}(11)-\mathrm{C}(12)-\mathrm{C}(17)-\mathrm{C}(16) & \\ & \\ & \end{array}$




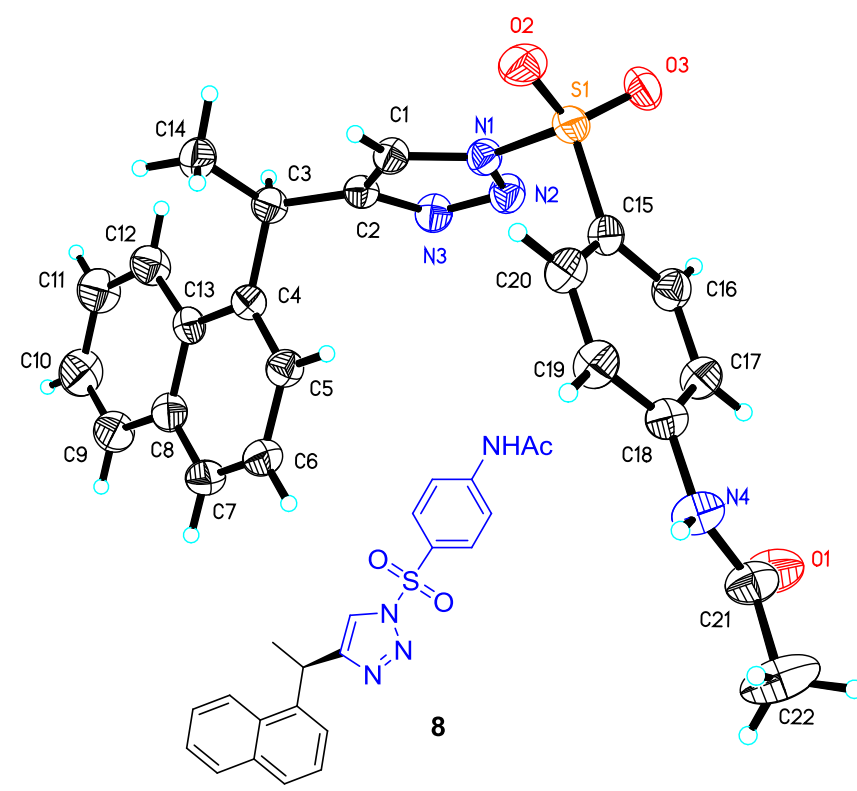

Table 1. Crystal data and structure refinement for mo_d8v18878_0m.

Identification code

Empirical formula

Formula weight

Temperature

Wavelength

Crystal system

Space group

Unit cell dimensions

Volume

Z

Density (calculated)

Absorption coefficient

$\mathrm{F}(000)$

Crystal size

Theta range for data collection

Index ranges

Reflections collected

Independent reflections

Completeness to theta $=25.242^{\circ}$

Absorption correction

Max. and min. transmission mo_d8v18878_0m

C22 H20 N4 O3 S

420.48

296(2) K

$0.71073 \AA$

Orthorhombic

P 212121

$$
\begin{array}{ll}
\mathrm{a}=9.7177(4) \AA & \alpha=90^{\circ} . \\
\mathrm{b}=10.0431(5) \AA & \beta=90^{\circ} . \\
\mathrm{c}=22.0386(10) \AA & \gamma=90^{\circ} .
\end{array}
$$

2150.88(17) $\AA^{3}$

4

$1.298 \mathrm{Mg} / \mathrm{m}^{3}$

$0.181 \mathrm{~mm}^{-1}$

880

$0.180 \times 0.110 \times 0.070 \mathrm{~mm}^{3}$

2.290 to $25.998^{\circ}$.

$-11<=\mathrm{h}<=11,-12<=\mathrm{k}<=12,-27<=\mathrm{l}<=27$

19750

$4215[\mathrm{R}(\mathrm{int})=0.0473]$

$99.8 \%$

Semi-empirical from equivalents

0.7456 and 0.6291 
Refinement method

Data / restraints / parameters

Goodness-of-fit on $\mathrm{F}^{2}$

Final R indices [I $>2 \operatorname{sigma}(\mathrm{I})]$

$\mathrm{R}$ indices (all data)

Absolute structure parameter

Extinction coefficient

Largest diff. peak and hole
Full-matrix least-squares on $\mathrm{F}^{2}$

4215 / 0 / 274

1.059

$\mathrm{R} 1=0.0431, \mathrm{wR} 2=0.1017$

$\mathrm{R} 1=0.0559, \mathrm{wR} 2=0.1118$

$0.06(4)$

$0.030(3)$

0.246 and -0.235 e. $\AA^{-3}$

Table 2. Atomic coordinates ( x 10 $)$ and equivalent isotropic displacement parameters $\left(\AA^{2} \times 10^{3}\right)$ for mo_d8v18878_0m. U(eq) is defined as one third of the trace of the orthogonalized $\mathrm{U}^{\mathrm{ij}}$ tensor.

\begin{tabular}{|c|c|c|c|c|}
\hline & $\mathrm{x}$ & $\mathrm{y}$ & $\mathrm{z}$ & $\mathrm{U}(\mathrm{eq})$ \\
\hline$S(1)$ & $2679(1)$ & $8055(1)$ & $3471(1)$ & $55(1)$ \\
\hline $\mathrm{O}(1)$ & $5507(3)$ & $10436(4)$ & $6088(1)$ & $83(1)$ \\
\hline $\mathrm{O}(2)$ & 1339(2) & $7530(2)$ & $3374(1)$ & 71(1) \\
\hline $\mathrm{O}(3)$ & $3257(3)$ & $9049(2)$ & $3089(1)$ & $73(1)$ \\
\hline $\mathrm{N}(1)$ & $3731(3)$ & $6706(2)$ & $3384(1)$ & $48(1)$ \\
\hline $\mathrm{N}(2)$ & $5100(3)$ & $6878(3)$ & $3328(1)$ & $56(1)$ \\
\hline $\mathrm{N}(3)$ & $5636(3)$ & $5694(3)$ & $3304(1)$ & $55(1)$ \\
\hline $\mathrm{N}(4)$ & $3389(3)$ & $9511(3)$ & $6043(1)$ & $61(1)$ \\
\hline $\mathrm{C}(1)$ & $3402(3)$ & $5396(3)$ & $3405(1)$ & $47(1)$ \\
\hline $\mathrm{C}(2)$ & $4624(3)$ & $4750(3)$ & $3347(1)$ & $45(1)$ \\
\hline$C(3)$ & 4940(3) & $3288(3)$ & $3316(1)$ & $52(1)$ \\
\hline $\mathrm{C}(4)$ & $5778(3)$ & $2825(3)$ & $3862(1)$ & $47(1)$ \\
\hline$C(5)$ & $5654(4)$ & $3436(4)$ & $4414(1)$ & $55(1)$ \\
\hline$C(6)$ & $6356(4)$ & 2983(4) & $4929(2)$ & $63(1)$ \\
\hline$C(7)$ & $7187(4)$ & $1910(4)$ & $4892(2)$ & $68(1)$ \\
\hline $\mathrm{C}(8)$ & $7372(3)$ & $1241(4)$ & $4334(2)$ & $59(1)$ \\
\hline $\mathrm{C}(9)$ & $8232(5)$ & $111(4)$ & $4284(2)$ & $82(1)$ \\
\hline$C(10)$ & $8385(5)$ & $-552(5)$ & $3753(2)$ & $91(1)$ \\
\hline $\mathrm{C}(11)$ & $7668(5)$ & $-119(4)$ & $3243(2)$ & $83(1)$ \\
\hline $\mathrm{C}(12)$ & $6824(4)$ & 959(4) & $3266(2)$ & $69(1)$ \\
\hline$C(13)$ & $6655(3)$ & $1685(3)$ & $3811(2)$ & $50(1)$ \\
\hline$C(14)$ & $3622(4)$ & $2475(3)$ & $3247(2)$ & $72(1)$ \\
\hline$C(15)$ & 2909(3) & $8470(3)$ & $4230(1)$ & $51(1)$ \\
\hline$C(16)$ & $3996(4)$ & $9285(3)$ & $4396(2)$ & $57(1)$ \\
\hline
\end{tabular}




$\begin{array}{lrrrr}\mathrm{C}(17) & 4186(4) & 9627(4) & 4990(2) & 56(1) \\ \mathrm{C}(18) & 3292(3) & 9163(3) & 5433(1) & 51(1) \\ \mathrm{C}(19) & 2207(4) & 8325(4) & 5263(2) & 60(1) \\ \mathrm{C}(20) & 2012(4) & 7978(4) & 4664(2) & 58(1) \\ \mathrm{C}(21) & 4436(4) & 10146(4) & 6332(2) & 66(1) \\ \mathrm{C}(22) & 4156(5) & 10434(7) & 6990(2) & 111(2)\end{array}$

Table 3. Bond lengths $[\AA]$ and angles $\left[^{\circ}\right]$ for mo_d8v18878_0m.

\begin{tabular}{ll}
\hline $\mathrm{S}(1)-\mathrm{O}(2)$ & $1.422(3)$ \\
$\mathrm{S}(1)-\mathrm{O}(3)$ & $1.421(3)$ \\
$\mathrm{S}(1)-\mathrm{N}(1)$ & $1.708(3)$ \\
$\mathrm{S}(1)-\mathrm{C}(15)$ & $1.737(3)$ \\
$\mathrm{O}(1)-\mathrm{C}(21)$ & $1.208(4)$ \\
$\mathrm{N}(1)-\mathrm{N}(2)$ & $1.348(4)$ \\
$\mathrm{N}(1)-\mathrm{C}(1)$ & $1.355(4)$ \\
$\mathrm{N}(2)-\mathrm{N}(3)$ & $1.300(4)$ \\
$\mathrm{N}(3)-\mathrm{C}(2)$ & $1.369(4)$ \\
$\mathrm{N}(4)-\mathrm{C}(21)$ & $1.359(5)$ \\
$\mathrm{N}(4)-\mathrm{C}(18)$ & $1.394(4)$ \\
$\mathrm{N}(4)-\mathrm{H}(4)$ & 0.8600 \\
$\mathrm{C}(1)-\mathrm{C}(2)$ & $1.359(4)$ \\
$\mathrm{C}(1)-\mathrm{H}(1)$ & 0.9300 \\
$\mathrm{C}(2)-\mathrm{C}(3)$ & $1.501(4)$ \\
$\mathrm{C}(3)-\mathrm{C}(4)$ & $1.525(4)$ \\
$\mathrm{C}(3)-\mathrm{C}(14)$ & $1.527(5)$ \\
$\mathrm{C}(3)-\mathrm{H}(3)$ & 0.9800 \\
$\mathrm{C}(4)-\mathrm{C}(5)$ & $1.367(4)$ \\
$\mathrm{C}(4)-\mathrm{C}(13)$ & $1.432(4)$ \\
$\mathrm{C}(5)-\mathrm{C}(6)$ & $1.400(5)$ \\
$\mathrm{C}(5)-\mathrm{H}(5)$ & 0.9300 \\
$\mathrm{C}(6)-\mathrm{C}(7)$ & $1.350(5)$ \\
$\mathrm{C}(6)-\mathrm{H}(6)$ & 0.9300 \\
$\mathrm{C}(7)-\mathrm{C}(8)$ & $1.413(5)$ \\
$\mathrm{C}(7)-\mathrm{H}(7)$ & 0.9300 \\
$\mathrm{C}(8)-\mathrm{C}(9)$ & $1.414(5)$ \\
$\mathrm{C}(8)-\mathrm{C}(13)$ & $1.418(5)$ \\
$\mathrm{C}(9)-\mathrm{C}(10)$ & \\
&
\end{tabular}




\begin{tabular}{|c|c|}
\hline $\mathrm{C}(9)-\mathrm{H}(9)$ & 0.9300 \\
\hline $\mathrm{C}(10)-\mathrm{C}(11)$ & $1.392(6)$ \\
\hline $\mathrm{C}(10)-\mathrm{H}(10)$ & 0.9300 \\
\hline $\mathrm{C}(11)-\mathrm{C}(12)$ & $1.359(5)$ \\
\hline $\mathrm{C}(11)-\mathrm{H}(11)$ & 0.9300 \\
\hline$C(12)-C(13)$ & $1.416(5)$ \\
\hline $\mathrm{C}(12)-\mathrm{H}(12)$ & 0.9300 \\
\hline $\mathrm{C}(14)-\mathrm{H}(14 \mathrm{~A})$ & 0.9600 \\
\hline $\mathrm{C}(14)-\mathrm{H}(14 \mathrm{~B})$ & 0.9600 \\
\hline $\mathrm{C}(14)-\mathrm{H}(14 \mathrm{C})$ & 0.9600 \\
\hline$C(15)-C(16)$ & $1.386(5)$ \\
\hline$C(15)-C(20)$ & $1.386(5)$ \\
\hline$C(16)-C(17)$ & $1.365(5)$ \\
\hline $\mathrm{C}(16)-\mathrm{H}(16)$ & 0.9300 \\
\hline $\mathrm{C}(17)-\mathrm{C}(18)$ & $1.387(5)$ \\
\hline $\mathrm{C}(17)-\mathrm{H}(17)$ & 0.9300 \\
\hline $\mathrm{C}(18)-\mathrm{C}(19)$ & $1.400(5)$ \\
\hline $\mathrm{C}(19)-\mathrm{C}(20)$ & $1.378(5)$ \\
\hline $\mathrm{C}(19)-\mathrm{H}(19)$ & 0.9300 \\
\hline $\mathrm{C}(20)-\mathrm{H}(20)$ & 0.9300 \\
\hline $\mathrm{C}(21)-\mathrm{C}(22)$ & $1.504(6)$ \\
\hline $\mathrm{C}(22)-\mathrm{H}(22 \mathrm{~A})$ & 0.9600 \\
\hline $\mathrm{C}(22)-\mathrm{H}(22 \mathrm{~B})$ & 0.9600 \\
\hline $\mathrm{C}(22)-\mathrm{H}(22 \mathrm{C})$ & 0.9600 \\
\hline $\mathrm{O}(2)-\mathrm{S}(1)-\mathrm{O}(3)$ & $122.18(17)$ \\
\hline $\mathrm{O}(2)-\mathrm{S}(1)-\mathrm{N}(1)$ & $103.70(13)$ \\
\hline $\mathrm{O}(3)-\mathrm{S}(1)-\mathrm{N}(1)$ & $104.72(15)$ \\
\hline $\mathrm{O}(2)-\mathrm{S}(1)-\mathrm{C}(15)$ & $110.63(17)$ \\
\hline $\mathrm{O}(3)-\mathrm{S}(1)-\mathrm{C}(15)$ & $110.53(16)$ \\
\hline $\mathrm{N}(1)-\mathrm{S}(1)-\mathrm{C}(15)$ & $102.83(14)$ \\
\hline $\mathrm{N}(2)-\mathrm{N}(1)-\mathrm{C}(1)$ & $111.2(3)$ \\
\hline $\mathrm{N}(2)-\mathrm{N}(1)-\mathrm{S}(1)$ & $119.9(2)$ \\
\hline $\mathrm{C}(1)-\mathrm{N}(1)-\mathrm{S}(1)$ & $128.7(2)$ \\
\hline $\mathrm{N}(3)-\mathrm{N}(2)-\mathrm{N}(1)$ & $106.3(3)$ \\
\hline $\mathrm{N}(2)-\mathrm{N}(3)-\mathrm{C}(2)$ & $110.1(2)$ \\
\hline $\mathrm{C}(21)-\mathrm{N}(4)-\mathrm{C}(18)$ & $128.4(3)$ \\
\hline $\mathrm{C}(21)-\mathrm{N}(4)-\mathrm{H}(4)$ & 115.8 \\
\hline
\end{tabular}




\begin{tabular}{|c|c|}
\hline $\mathrm{C}(18)-\mathrm{N}(4)-\mathrm{H}(4)$ & 115.8 \\
\hline $\mathrm{N}(1)-\mathrm{C}(1)-\mathrm{C}(2)$ & $104.7(3)$ \\
\hline $\mathrm{N}(1)-\mathrm{C}(1)-\mathrm{H}(1)$ & 127.6 \\
\hline $\mathrm{C}(2)-\mathrm{C}(1)-\mathrm{H}(1)$ & 127.6 \\
\hline $\mathrm{C}(1)-\mathrm{C}(2)-\mathrm{N}(3)$ & 107.7(3) \\
\hline $\mathrm{C}(1)-\mathrm{C}(2)-\mathrm{C}(3)$ & $130.5(3)$ \\
\hline $\mathrm{N}(3)-\mathrm{C}(2)-\mathrm{C}(3)$ & $121.8(3)$ \\
\hline $\mathrm{C}(2)-\mathrm{C}(3)-\mathrm{C}(4)$ & $111.9(3)$ \\
\hline$C(2)-C(3)-C(14)$ & $110.9(3)$ \\
\hline$C(4)-C(3)-C(14)$ & $111.3(3)$ \\
\hline $\mathrm{C}(2)-\mathrm{C}(3)-\mathrm{H}(3)$ & 107.5 \\
\hline $\mathrm{C}(4)-\mathrm{C}(3)-\mathrm{H}(3)$ & 107.5 \\
\hline $\mathrm{C}(14)-\mathrm{C}(3)-\mathrm{H}(3)$ & 107.5 \\
\hline$C(5)-C(4)-C(13)$ & $118.7(3)$ \\
\hline$C(5)-C(4)-C(3)$ & 121.1(3) \\
\hline$C(13)-C(4)-C(3)$ & $120.0(3)$ \\
\hline$C(4)-C(5)-C(6)$ & $122.2(3)$ \\
\hline $\mathrm{C}(4)-\mathrm{C}(5)-\mathrm{H}(5)$ & 118.9 \\
\hline $\mathrm{C}(6)-\mathrm{C}(5)-\mathrm{H}(5)$ & 118.9 \\
\hline$C(7)-C(6)-C(5)$ & $120.2(3)$ \\
\hline $\mathrm{C}(7)-\mathrm{C}(6)-\mathrm{H}(6)$ & 119.9 \\
\hline $\mathrm{C}(5)-\mathrm{C}(6)-\mathrm{H}(6)$ & 119.9 \\
\hline$C(6)-C(7)-C(8)$ & $120.5(3)$ \\
\hline $\mathrm{C}(6)-\mathrm{C}(7)-\mathrm{H}(7)$ & 119.8 \\
\hline $\mathrm{C}(8)-\mathrm{C}(7)-\mathrm{H}(7)$ & 119.8 \\
\hline $\mathrm{C}(9)-\mathrm{C}(8)-\mathrm{C}(7)$ & $121.6(4)$ \\
\hline $\mathrm{C}(9)-\mathrm{C}(8)-\mathrm{C}(13)$ & $118.6(3)$ \\
\hline$C(7)-C(8)-C(13)$ & $119.7(3)$ \\
\hline$C(10)-C(9)-C(8)$ & $121.8(4)$ \\
\hline $\mathrm{C}(10)-\mathrm{C}(9)-\mathrm{H}(9)$ & 119.1 \\
\hline $\mathrm{C}(8)-\mathrm{C}(9)-\mathrm{H}(9)$ & 119.1 \\
\hline $\mathrm{C}(9)-\mathrm{C}(10)-\mathrm{C}(11)$ & $119.3(4)$ \\
\hline $\mathrm{C}(9)-\mathrm{C}(10)-\mathrm{H}(10)$ & 120.3 \\
\hline $\mathrm{C}(11)-\mathrm{C}(10)-\mathrm{H}(10)$ & 120.3 \\
\hline $\mathrm{C}(12)-\mathrm{C}(11)-\mathrm{C}(10)$ & $121.4(4)$ \\
\hline $\mathrm{C}(12)-\mathrm{C}(11)-\mathrm{H}(11)$ & 119.3 \\
\hline $\mathrm{C}(10)-\mathrm{C}(11)-\mathrm{H}(11)$ & 119.3 \\
\hline $\mathrm{C}(11)-\mathrm{C}(12)-\mathrm{C}(13)$ & $120.8(4)$ \\
\hline
\end{tabular}




\begin{tabular}{|c|c|}
\hline $\mathrm{C}(11)-\mathrm{C}(12)-\mathrm{H}(12)$ & 119.6 \\
\hline $\mathrm{C}(13)-\mathrm{C}(12)-\mathrm{H}(12)$ & 119.6 \\
\hline$C(12)-C(13)-C(8)$ & 118.1(3) \\
\hline$C(12)-C(13)-C(4)$ & $123.2(3)$ \\
\hline $\mathrm{C}(8)-\mathrm{C}(13)-\mathrm{C}(4)$ & $118.7(3)$ \\
\hline $\mathrm{C}(3)-\mathrm{C}(14)-\mathrm{H}(14 \mathrm{~A})$ & 109.5 \\
\hline $\mathrm{C}(3)-\mathrm{C}(14)-\mathrm{H}(14 \mathrm{~B})$ & 109.5 \\
\hline $\mathrm{H}(14 \mathrm{~A})-\mathrm{C}(14)-\mathrm{H}(14 \mathrm{~B})$ & 109.5 \\
\hline $\mathrm{C}(3)-\mathrm{C}(14)-\mathrm{H}(14 \mathrm{C})$ & 109.5 \\
\hline $\mathrm{H}(14 \mathrm{~A})-\mathrm{C}(14)-\mathrm{H}(14 \mathrm{C})$ & 109.5 \\
\hline $\mathrm{H}(14 \mathrm{~B})-\mathrm{C}(14)-\mathrm{H}(14 \mathrm{C})$ & 109.5 \\
\hline$C(16)-C(15)-C(20)$ & $120.4(3)$ \\
\hline$C(16)-C(15)-S(1)$ & $119.7(3)$ \\
\hline$C(20)-C(15)-S(1)$ & $119.9(3)$ \\
\hline$C(17)-C(16)-C(15)$ & $120.4(3)$ \\
\hline $\mathrm{C}(17)-\mathrm{C}(16)-\mathrm{H}(16)$ & 119.8 \\
\hline $\mathrm{C}(15)-\mathrm{C}(16)-\mathrm{H}(16)$ & 119.8 \\
\hline$C(16)-C(17)-C(18)$ & $120.3(3)$ \\
\hline $\mathrm{C}(16)-\mathrm{C}(17)-\mathrm{H}(17)$ & 119.8 \\
\hline $\mathrm{C}(18)-\mathrm{C}(17)-\mathrm{H}(17)$ & 119.8 \\
\hline $\mathrm{C}(17)-\mathrm{C}(18)-\mathrm{N}(4)$ & $123.5(3)$ \\
\hline$C(17)-C(18)-C(19)$ & 119.1(3) \\
\hline $\mathrm{N}(4)-\mathrm{C}(18)-\mathrm{C}(19)$ & $117.4(3)$ \\
\hline$C(20)-C(19)-C(18)$ & $120.8(3)$ \\
\hline $\mathrm{C}(20)-\mathrm{C}(19)-\mathrm{H}(19)$ & 119.6 \\
\hline $\mathrm{C}(18)-\mathrm{C}(19)-\mathrm{H}(19)$ & 119.6 \\
\hline$C(19)-C(20)-C(15)$ & 119.1(3) \\
\hline $\mathrm{C}(19)-\mathrm{C}(20)-\mathrm{H}(20)$ & 120.5 \\
\hline $\mathrm{C}(15)-\mathrm{C}(20)-\mathrm{H}(20)$ & 120.5 \\
\hline $\mathrm{O}(1)-\mathrm{C}(21)-\mathrm{N}(4)$ & $123.4(3)$ \\
\hline $\mathrm{O}(1)-\mathrm{C}(21)-\mathrm{C}(22)$ & $122.6(4)$ \\
\hline $\mathrm{N}(4)-\mathrm{C}(21)-\mathrm{C}(22)$ & $114.0(4)$ \\
\hline $\mathrm{C}(21)-\mathrm{C}(22)-\mathrm{H}(22 \mathrm{~A})$ & 109.5 \\
\hline $\mathrm{C}(21)-\mathrm{C}(22)-\mathrm{H}(22 \mathrm{~B})$ & 109.5 \\
\hline $\mathrm{H}(22 \mathrm{~A})-\mathrm{C}(22)-\mathrm{H}(22 \mathrm{~B})$ & 109.5 \\
\hline $\mathrm{C}(21)-\mathrm{C}(22)-\mathrm{H}(22 \mathrm{C})$ & 109.5 \\
\hline $\mathrm{H}(22 \mathrm{~A})-\mathrm{C}(22)-\mathrm{H}(22 \mathrm{C})$ & 109.5 \\
\hline $\mathrm{H}(22 \mathrm{~B})-\mathrm{C}(22)-\mathrm{H}(22 \mathrm{C})$ & 109.5 \\
\hline
\end{tabular}


Symmetry transformations used to generate equivalent atoms:

Table 4. Anisotropic displacement parameters $\left(\AA^{2} \mathrm{x} 10^{3}\right)$ for mo_d8v18878_0m. The anisotropic displacement factor exponent takes the form: $-2 \pi^{2}\left[h^{2} a^{* 2} U^{11}+\ldots+2 h k a * b * U^{12}\right]$

\begin{tabular}{|c|c|c|c|c|c|c|}
\hline & $\mathrm{U}^{11}$ & $\mathrm{U}^{22}$ & $\mathrm{U}^{33}$ & $\mathrm{U}^{23}$ & $\mathrm{U}^{13}$ & $\mathrm{U}^{12}$ \\
\hline$S(1)$ & $64(1)$ & $46(1)$ & $55(1)$ & $-4(1)$ & $-11(1)$ & $12(1)$ \\
\hline $\mathrm{O}(1)$ & $50(2)$ & $129(3)$ & $69(2)$ & $-7(2)$ & $-4(1)$ & $-12(2)$ \\
\hline $\mathrm{O}(2)$ & $57(2)$ & $72(2)$ & $83(2)$ & $-17(1)$ & $-22(1)$ & $21(1)$ \\
\hline $\mathrm{O}(3)$ & $113(2)$ & $48(1)$ & $58(1)$ & $9(1)$ & $-7(1)$ & $9(1)$ \\
\hline $\mathrm{N}(1)$ & $46(2)$ & $44(1)$ & $53(2)$ & $-3(1)$ & $-2(1)$ & $3(1)$ \\
\hline $\mathrm{N}(2)$ & $51(2)$ & $51(2)$ & $67(2)$ & $-2(1)$ & $-2(1)$ & $-3(1)$ \\
\hline $\mathrm{N}(3)$ & $46(2)$ & $56(2)$ & $64(2)$ & $-1(1)$ & $0(1)$ & $0(1)$ \\
\hline $\mathrm{N}(4)$ & $45(2)$ & $86(2)$ & $52(2)$ & $-9(2)$ & $4(1)$ & $-2(2)$ \\
\hline $\mathrm{C}(1)$ & $42(2)$ & $46(2)$ & $52(2)$ & $-3(1)$ & $-1(1)$ & $1(1)$ \\
\hline $\mathrm{C}(2)$ & $46(2)$ & $48(2)$ & $42(2)$ & $0(1)$ & $-1(1)$ & $3(1)$ \\
\hline $\mathrm{C}(3)$ & $57(2)$ & $49(2)$ & $49(2)$ & $-4(1)$ & $2(1)$ & $9(2)$ \\
\hline $\mathrm{C}(4)$ & $46(2)$ & $50(2)$ & $46(2)$ & $0(1)$ & $5(1)$ & $2(1)$ \\
\hline $\mathrm{C}(5)$ & $56(2)$ & $56(2)$ & $53(2)$ & $-2(2)$ & $2(2)$ & $1(2)$ \\
\hline$C(6)$ & $73(2)$ & $65(2)$ & $52(2)$ & $-4(2)$ & $-4(2)$ & $-5(2)$ \\
\hline$C(7)$ & $69(2)$ & $71(2)$ & $63(2)$ & $6(2)$ & $-18(2)$ & $-6(2)$ \\
\hline $\mathrm{C}(8)$ & $46(2)$ & $55(2)$ & $75(2)$ & $2(2)$ & $-6(2)$ & $1(2)$ \\
\hline $\mathrm{C}(9)$ & $73(3)$ & $67(3)$ & $104(3)$ & $5(2)$ & $-29(2)$ & $16(2)$ \\
\hline$C(10)$ & $81(3)$ & $73(3)$ & $118(4)$ & $-13(3)$ & $-9(3)$ & $29(2)$ \\
\hline $\mathrm{C}(11)$ & $92(3)$ & $72(3)$ & $85(3)$ & $-13(2)$ & $7(2)$ & $25(2)$ \\
\hline$C(12)$ & $76(3)$ & $63(2)$ & $67(2)$ & $-3(2)$ & $4(2)$ & $18(2)$ \\
\hline $\mathrm{C}(13)$ & $48(2)$ & $45(2)$ & $58(2)$ & $3(1)$ & $4(1)$ & $2(1)$ \\
\hline $\mathrm{C}(14)$ & $81(3)$ & $49(2)$ & $86(3)$ & $-4(2)$ & $-22(2)$ & $1(2)$ \\
\hline$C(15)$ & $57(2)$ & $42(2)$ & $54(2)$ & $-3(1)$ & $-3(2)$ & $5(2)$ \\
\hline$C(16)$ & $59(2)$ & $54(2)$ & $57(2)$ & $-1(2)$ & $4(2)$ & $-5(2)$ \\
\hline$C(17)$ & $50(2)$ & $60(2)$ & $58(2)$ & $-5(2)$ & $3(2)$ & $-5(2)$ \\
\hline $\mathrm{C}(18)$ & $43(2)$ & $58(2)$ & $52(2)$ & $-3(2)$ & $2(1)$ & $6(2)$ \\
\hline $\mathrm{C}(19)$ & $52(2)$ & $66(2)$ & $63(2)$ & $-4(2)$ & $10(2)$ & $-10(2)$ \\
\hline$C(20)$ & $56(2)$ & $58(2)$ & $62(2)$ & $-6(2)$ & $1(2)$ & $-6(2)$ \\
\hline $\mathrm{C}(21)$ & $44(2)$ & $91(3)$ & $64(2)$ & $-11(2)$ & $-6(2)$ & $7(2)$ \\
\hline $\mathrm{C}(22)$ & $71(3)$ & 197(6) & $66(3)$ & $-44(3)$ & $1(2)$ & $-7(3)$ \\
\hline
\end{tabular}


Table 5. Hydrogen coordinates ( x 104) and isotropic displacement parameters $\left(\AA^{2} \times 10^{3}\right)$ for mo_d8v18878_0m.

\begin{tabular}{|c|c|c|c|c|}
\hline & $\mathrm{x}$ & $\mathrm{y}$ & $\mathrm{z}$ & $\mathrm{U}(\mathrm{eq})$ \\
\hline $\mathrm{H}(4)$ & 2697 & 9296 & 6266 & 73 \\
\hline $\mathrm{H}(1)$ & 2533 & 5021 & 3450 & 56 \\
\hline $\mathrm{H}(3)$ & 5500 & 3138 & 2953 & 62 \\
\hline $\mathrm{H}(5)$ & 5086 & 4177 & 4448 & 66 \\
\hline $\mathrm{H}(6)$ & 6248 & 3423 & 5297 & 76 \\
\hline $\mathrm{H}(7)$ & 7642 & 1608 & 5237 & 81 \\
\hline $\mathrm{H}(9)$ & 8705 & -186 & 4625 & 98 \\
\hline $\mathrm{H}(10)$ & 8962 & -1289 & 3729 & 109 \\
\hline $\mathrm{H}(11)$ & 7769 & -577 & 2878 & 100 \\
\hline $\mathrm{H}(12)$ & 6352 & 1222 & 2919 & 82 \\
\hline $\mathrm{H}(14 \mathrm{~A})$ & 3031 & 2637 & 3588 & 108 \\
\hline $\mathrm{H}(14 \mathrm{~B})$ & 3848 & 1545 & 3230 & 108 \\
\hline $\mathrm{H}(14 \mathrm{C})$ & 3160 & 2731 & 2881 & 108 \\
\hline $\mathrm{H}(16)$ & 4599 & 9601 & 4102 & 68 \\
\hline $\mathrm{H}(17)$ & 4920 & 10172 & 5098 & 67 \\
\hline $\mathrm{H}(19)$ & 1611 & 7998 & 5558 & 72 \\
\hline $\mathrm{H}(20)$ & 1290 & 7422 & 4553 & 70 \\
\hline $\mathrm{H}(22 \mathrm{~A})$ & 4230 & 11375 & 7062 & 167 \\
\hline $\mathrm{H}(22 \mathrm{~B})$ & 4814 & 9972 & 7238 & 167 \\
\hline $\mathrm{H}(22 \mathrm{C})$ & 3244 & 10140 & 7092 & 167 \\
\hline
\end{tabular}

Table 6. Torsion angles $\left[{ }^{\circ}\right]$ for mo_d8v18878_0m.

\begin{tabular}{lc}
\hline $\mathrm{O}(2)-\mathrm{S}(1)-\mathrm{N}(1)-\mathrm{N}(2)$ & $167.0(2)$ \\
$\mathrm{O}(3)-\mathrm{S}(1)-\mathrm{N}(1)-\mathrm{N}(2)$ & $37.9(3)$ \\
$\mathrm{C}(15)-\mathrm{S}(1)-\mathrm{N}(1)-\mathrm{N}(2)$ & $-77.7(3)$ \\
$\mathrm{O}(2)-\mathrm{S}(1)-\mathrm{N}(1)-\mathrm{C}(1)$ & $-18.4(3)$ \\
$\mathrm{O}(3)-\mathrm{S}(1)-\mathrm{N}(1)-\mathrm{C}(1)$ & $-147.5(3)$ \\
$\mathrm{C}(15)-\mathrm{S}(1)-\mathrm{N}(1)-\mathrm{C}(1)$ & $96.9(3)$ \\
$\mathrm{C}(1)-\mathrm{N}(1)-\mathrm{N}(2)-\mathrm{N}(3)$ & $0.9(4)$ \\
$\mathrm{S}(1)-\mathrm{N}(1)-\mathrm{N}(2)-\mathrm{N}(3)$ & $176.4(2)$ \\
$\mathrm{N}(1)-\mathrm{N}(2)-\mathrm{N}(3)-\mathrm{C}(2)$ & $-0.2(3)$
\end{tabular}




\begin{tabular}{|c|c|}
\hline $\mathrm{N}(2)-\mathrm{N}(1)-\mathrm{C}(1)-\mathrm{C}(2)$ & $-1.2(4)$ \\
\hline $\mathrm{S}(1)-\mathrm{N}(1)-\mathrm{C}(1)-\mathrm{C}(2)$ & $-176.2(2)$ \\
\hline $\mathrm{N}(1)-\mathrm{C}(1)-\mathrm{C}(2)-\mathrm{N}(3)$ & $1.0(3)$ \\
\hline $\mathrm{N}(1)-\mathrm{C}(1)-\mathrm{C}(2)-\mathrm{C}(3)$ & $-177.7(3)$ \\
\hline $\mathrm{N}(2)-\mathrm{N}(3)-\mathrm{C}(2)-\mathrm{C}(1)$ & $-0.5(3)$ \\
\hline $\mathrm{N}(2)-\mathrm{N}(3)-\mathrm{C}(2)-\mathrm{C}(3)$ & 178.4(3) \\
\hline $\mathrm{C}(1)-\mathrm{C}(2)-\mathrm{C}(3)-\mathrm{C}(4)$ & $-114.8(4)$ \\
\hline $\mathrm{N}(3)-\mathrm{C}(2)-\mathrm{C}(3)-\mathrm{C}(4)$ & $66.6(4)$ \\
\hline$C(1)-C(2)-C(3)-C(14)$ & $10.0(5)$ \\
\hline $\mathrm{N}(3)-\mathrm{C}(2)-\mathrm{C}(3)-\mathrm{C}(14)$ & $-168.6(3)$ \\
\hline $\mathrm{C}(2)-\mathrm{C}(3)-\mathrm{C}(4)-\mathrm{C}(5)$ & $29.8(4)$ \\
\hline $\mathrm{C}(14)-\mathrm{C}(3)-\mathrm{C}(4)-\mathrm{C}(5)$ & $-94.9(4)$ \\
\hline $\mathrm{C}(2)-\mathrm{C}(3)-\mathrm{C}(4)-\mathrm{C}(13)$ & $-153.8(3)$ \\
\hline $\mathrm{C}(14)-\mathrm{C}(3)-\mathrm{C}(4)-\mathrm{C}(13)$ & $81.6(4)$ \\
\hline $\mathrm{C}(13)-\mathrm{C}(4)-\mathrm{C}(5)-\mathrm{C}(6)$ & $0.0(5)$ \\
\hline$C(3)-C(4)-C(5)-C(6)$ & 176.4(3) \\
\hline$C(4)-C(5)-C(6)-C(7)$ & $0.0(6)$ \\
\hline$C(5)-C(6)-C(7)-C(8)$ & $0.7(6)$ \\
\hline $\mathrm{C}(6)-\mathrm{C}(7)-\mathrm{C}(8)-\mathrm{C}(9)$ & $-179.7(4)$ \\
\hline $\mathrm{C}(6)-\mathrm{C}(7)-\mathrm{C}(8)-\mathrm{C}(13)$ & $-1.3(6)$ \\
\hline $\mathrm{C}(7)-\mathrm{C}(8)-\mathrm{C}(9)-\mathrm{C}(10)$ & $178.4(4)$ \\
\hline $\mathrm{C}(13)-\mathrm{C}(8)-\mathrm{C}(9)-\mathrm{C}(10)$ & $0.0(6)$ \\
\hline $\mathrm{C}(8)-\mathrm{C}(9)-\mathrm{C}(10)-\mathrm{C}(11)$ & $-0.6(7)$ \\
\hline$C(9)-C(10)-C(11)-C(12)$ & $0.4(8)$ \\
\hline $\mathrm{C}(10)-\mathrm{C}(11)-\mathrm{C}(12)-\mathrm{C}(13)$ & $0.5(7)$ \\
\hline $\mathrm{C}(11)-\mathrm{C}(12)-\mathrm{C}(13)-\mathrm{C}(8)$ & $-1.1(6)$ \\
\hline $\mathrm{C}(11)-\mathrm{C}(12)-\mathrm{C}(13)-\mathrm{C}(4)$ & $-179.9(4)$ \\
\hline $\mathrm{C}(9)-\mathrm{C}(8)-\mathrm{C}(13)-\mathrm{C}(12)$ & $0.8(5)$ \\
\hline $\mathrm{C}(7)-\mathrm{C}(8)-\mathrm{C}(13)-\mathrm{C}(12)$ & $-177.6(3)$ \\
\hline $\mathrm{C}(9)-\mathrm{C}(8)-\mathrm{C}(13)-\mathrm{C}(4)$ & $179.6(3)$ \\
\hline $\mathrm{C}(7)-\mathrm{C}(8)-\mathrm{C}(13)-\mathrm{C}(4)$ & $1.3(5)$ \\
\hline $\mathrm{C}(5)-\mathrm{C}(4)-\mathrm{C}(13)-\mathrm{C}(12)$ & $178.2(3)$ \\
\hline$C(3)-C(4)-C(13)-C(12)$ & $1.7(5)$ \\
\hline $\mathrm{C}(5)-\mathrm{C}(4)-\mathrm{C}(13)-\mathrm{C}(8)$ & $-0.6(5)$ \\
\hline $\mathrm{C}(3)-\mathrm{C}(4)-\mathrm{C}(13)-\mathrm{C}(8)$ & $-177.1(3)$ \\
\hline $\mathrm{O}(2)-\mathrm{S}(1)-\mathrm{C}(15)-\mathrm{C}(16)$ & $-164.4(3)$ \\
\hline $\mathrm{O}(3)-\mathrm{S}(1)-\mathrm{C}(15)-\mathrm{C}(16)$ & $-25.9(3)$ \\
\hline $\mathrm{N}(1)-\mathrm{S}(1)-\mathrm{C}(15)-\mathrm{C}(16)$ & $85.4(3)$ \\
\hline
\end{tabular}




$\begin{array}{lc}\mathrm{O}(2)-\mathrm{S}(1)-\mathrm{C}(15)-\mathrm{C}(20) & 16.1(3) \\ \mathrm{O}(3)-\mathrm{S}(1)-\mathrm{C}(15)-\mathrm{C}(20) & 154.6(3) \\ \mathrm{N}(1)-\mathrm{S}(1)-\mathrm{C}(15)-\mathrm{C}(20) & -94.1(3) \\ \mathrm{C}(20)-\mathrm{C}(15)-\mathrm{C}(16)-\mathrm{C}(17) & -0.8(5) \\ \mathrm{S}(1)-\mathrm{C}(15)-\mathrm{C}(16)-\mathrm{C}(17) & 179.7(3) \\ \mathrm{C}(15)-\mathrm{C}(16)-\mathrm{C}(17)-\mathrm{C}(18) & -0.1(5) \\ \mathrm{C}(16)-\mathrm{C}(17)-\mathrm{C}(18)-\mathrm{N}(4) & -177.4(3) \\ \mathrm{C}(16)-\mathrm{C}(17)-\mathrm{C}(18)-\mathrm{C}(19) & 1.0(5) \\ \mathrm{C}(21)-\mathrm{N}(4)-\mathrm{C}(18)-\mathrm{C}(17) & -11.5(6) \\ \mathrm{C}(21)-\mathrm{N}(4)-\mathrm{C}(18)-\mathrm{C}(19) & 170.0(4) \\ \mathrm{C}(17)-\mathrm{C}(18)-\mathrm{C}(19)-\mathrm{C}(20) & -1.0(5) \\ \mathrm{N}(4)-\mathrm{C}(18)-\mathrm{C}(19)-\mathrm{C}(20) & 177.5(3) \\ \mathrm{C}(18)-\mathrm{C}(19)-\mathrm{C}(20)-\mathrm{C}(15) & 0.1(5) \\ \mathrm{C}(16)-\mathrm{C}(15)-\mathrm{C}(20)-\mathrm{C}(19) & 0.8(5) \\ \mathrm{S}(1)-\mathrm{C}(15)-\mathrm{C}(20)-\mathrm{C}(19) & -179.6(3) \\ \mathrm{C}(18)-\mathrm{N}(4)-\mathrm{C}(21)-\mathrm{O}(1) & -4.7(7) \\ \mathrm{C}(18)-\mathrm{N}(4)-\mathrm{C}(21)-\mathrm{C}(22) & 176.5(4)\end{array}$

Symmetry transformations used to generate equivalent atoms:

Table 7. Hydrogen bonds for mo_d8v18878_0m [Å and $\left.{ }^{\circ}\right]$.

\begin{tabular}{lllll}
\hline D-H...A & d(D-H) & d(H...A & d(D...A $)$ & $<($ DHA $)$
\end{tabular}

\section{DFT Calculations}

\section{Computational details}

All of the calculations were performed using the Gaussian 09 D.01 program. ${ }^{\text {S6 }}$ Structures were optimized at the $(\mathrm{U}) \mathrm{wB} 97 \mathrm{XD}^{\mathrm{S} 7}$ level of density functional theory. The keyword "int=ultrafine" in the Gaussian 09 D.01 program was used to increase the accuracy of the calculations. The SDD basis set ${ }^{\mathrm{S} 8}$ with $\mathrm{f}$ polarization $\left(\zeta_{\mathrm{f}}=3.525\right)^{\mathrm{S} 9}$ was used for $\mathrm{Cu}$, while the 6-31G(d) basis set was used for all other atoms. Frequency calculations have been performed to verify the optimized structures as local minima or transition states and to obtain Gibbs free energy at $298 \mathrm{~K}$. Intrinsic reaction coordinate (IRC) calculations ${ }^{\mathrm{S} 10}$ were carried out to make sure that every transition state links relevant intermediates. The more accurate relative 
stabilities were further refined by carrying out single-point energy calculations using (U)wB97XD functional, def2TZVP basis set for $\mathrm{Cu}^{\mathrm{S} 11}$, and 6-311G(d,p) basis set for all nonmental atoms. CPCM solvation model ${ }^{\mathrm{S} 12-13}$ with perfluorobenzene as solvent was employed in the single-point energy calculations.

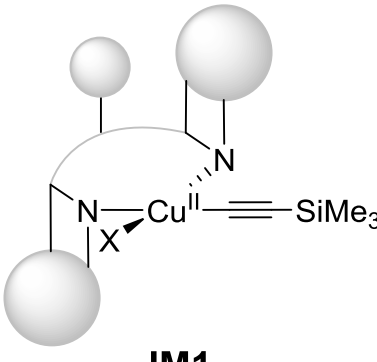

IM1

0.0

$(0.0)$

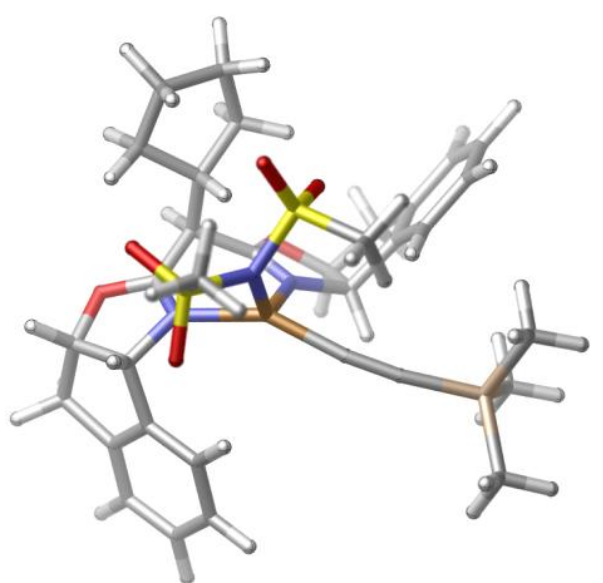

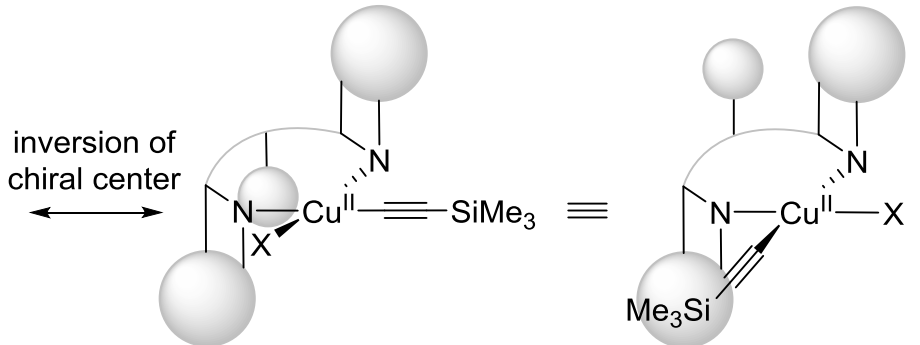

IM3

7.9

(10.9)

Figure S1. Comparison of the two diastereomers IM1 and IM3 calculated for $\mathrm{L}^{*} \mathrm{Cu}($ alkynyl)(bismethylsulfonamide).

Notes on Figure S1: In the main text, we presented the calculation results considering how the chiral $\mathrm{L}^{*}$ CuII-alkynyl $\left(\mathrm{L}^{*}=\mathbf{L 9}\right.$, IM1) reacts with a benzyl radical. As a diastereomer of IM1, IM3 was also calculated. IM3 is lying $7.9 \mathrm{kcal} / \mathrm{mol}$ higher than IM1, and thus we do not consider further the corresponding reaction from IM3 in view of the fact that the barriers from IM1 are relatively low. 


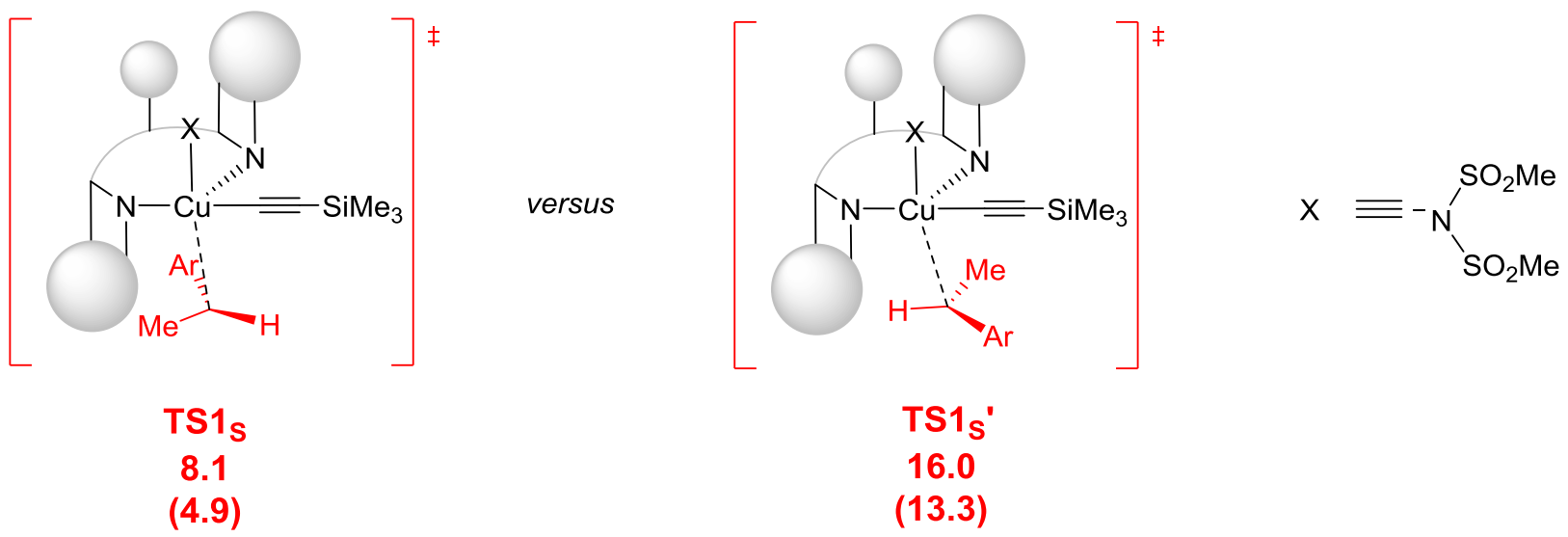

Figure S2. Transition state structures (leading to the $S$ product) in different conformations. The relative free energies and electronic energies (in parentheses) are given in $\mathrm{kcal} / \mathrm{mol}$.

Notes on Figure S2: The conformations of the transition states presented in the main text are the most stable ones. Here, we showed a higher energy conformation, TS1 $\mathbf{s}^{\prime}$, which is lying $7.9 \mathrm{kcal} / \mathrm{mol}$ higher in free energy than $\mathbf{T S} \mathbf{1}_{\mathbf{S}}$ (presented in the main text).

\section{XYZ coordinate}

IM1+int1

\begin{tabular}{lrrr}
$E=$ & -5087.967606 & \multicolumn{2}{c}{$G=-5087.158135$} \\
$O$ & 3.36126800 & 2.44476000 & 0.71758700 \\
$O$ & -0.80053000 & 1.93179500 & 1.80738000 \\
$N$ & 2.33656400 & 1.35907400 & -0.94852200 \\
$N$ & -0.37857800 & 0.87453500 & -0.11616400 \\
$\mathrm{C}$ & 3.75826200 & 0.98853100 & -1.10210500 \\
$\mathrm{C}$ & 4.00337600 & -0.48593500 & -0.93425800 \\
$\mathrm{C}$ & 3.70768600 & -1.50182000 & -1.83808500 \\
$\mathrm{C}$ & 4.00567700 & -2.81351900 & -1.47633900 \\
$\mathrm{C}$ & 4.56978400 & -3.09565200 & -0.23091600 \\
$\mathrm{C}$ & 4.87017300 & -2.07068000 & 0.66441300 \\
$\mathrm{C}$ & 4.59454600 & -0.75745300 & 0.29809100 \\
$\mathrm{C}$ & 4.87397600 & 0.50748500 & 1.07809100 \\
$\mathrm{C}$ & 4.42338000 & 1.64395600 & 0.13624600 \\
$\mathrm{C}$ & 2.24757000 & 2.16003300 & 0.04519700 \\
$\mathrm{C}$ & -0.08613300 & 1.81983400 & 0.69595600 \\
$\mathrm{C}$ & -1.65917300 & 0.75708000 & 1.87241400 \\
$\mathrm{C}$ & -3.10664200 & 1.16668900 & 2.13108100 \\
$\mathrm{C}$ & -3.72653500 & 1.18700500 & 0.75645900 \\
$\mathrm{C}$ & -4.98660600 & 1.65461500 & 0.39839100 \\
$\mathrm{C}$ & -5.39126000 & 1.55234400 & -0.92971100 \\
$\mathrm{C}$ & -4.54343500 & 0.98775700 & -1.88303200 \\
$\mathrm{C}$ & -3.28249000 & 0.52101200 & -1.52441400 \\
$\mathrm{C}$ & -2.87832400 & 0.62553300 & -0.19806700 \\
$\mathrm{C}$ & -1.56609000 & 0.19142900 & 0.43017700 \\
$\mathrm{H}$ & 4.10837200 & 1.36965000 & -2.06076300 \\
$\mathrm{H}$ & 3.26577900 & -1.26254000 & -2.80066700 \\
$\mathrm{H}$ & 3.78822100 & -3.62398500 & -2.16505500 \\
& & &
\end{tabular}




\begin{tabular}{|c|c|c|c|}
\hline H & 600 & 9600 & 2000 \\
\hline $\boldsymbol{n}$ & 5.31776200 & -2.29622700 & 1.62827900 \\
\hline H & 4.31596600 & 0.54285100 & 2.01981900 \\
\hline H & 5.93525400 & 0.60153900 & 1.33066900 \\
\hline H & 5.22643800 & 2.33684700 & -0.11153300 \\
\hline $\mathrm{H}$ & -1.23551500 & 0.11492800 & 2.64381500 \\
\hline $\mathrm{H}$ & -3.59243500 & 0.42476300 & 2.77609100 \\
\hline $\mathrm{H}$ & -3.15393200 & 2.13001300 & 2.64849300 \\
\hline $\mathrm{H}$ & -5.64459400 & 2.09469500 & 1.14301400 \\
\hline H & -6.37056000 & 1.91745800 & -1.22528300 \\
\hline H & -4.86395100 & 0.92125800 & -2.91880800 \\
\hline $\mathrm{H}$ & -2.61499300 & 0.09382800 & -2.26064000 \\
\hline H & -1.40729400 & -0.88884900 & 0.38297800 \\
\hline C & 0.98970200 & 2.84168700 & 0.48330200 \\
\hline $\mathrm{Cu}$ & 0.69548500 & 0.52769700 & -1.86749900 \\
\hline C & -0.29659300 & -1.01256100 & -2.39377700 \\
\hline C & -1.07486900 & -1.94006900 & -2.59358500 \\
\hline $\mathrm{Si}$ & -2.40592500 & -3.19582800 & -2.61705500 \\
\hline C & -3.06773600 & -3.34544900 & -0.85696800 \\
\hline $\mathrm{H}$ & -2.27159700 & -3.58452900 & -0.14212000 \\
\hline H & -3.51183400 & -2.39061200 & -0.55051300 \\
\hline H & -3.84135000 & -4.11846200 & -0.77816100 \\
\hline & -3.78882400 & -2.59549500 & -3.74909400 \\
\hline H & -4.60002900 & -3.33128200 & -3.80276500 \\
\hline H & -4.21277300 & -1.65693800 & -3.37486500 \\
\hline $\mathrm{H}$ & -3.42178700 & -2.42302400 & -4.76704300 \\
\hline C & -1.75885700 & -4.85043000 & -3.24435400 \\
\hline $\mathrm{H}$ & -1.38458600 & -4.75410600 & -4.26961300 \\
\hline H & -0.93827400 & -5.22761300 & -2.62514100 \\
\hline H & -2.55553700 & -5.60387300 & -3.24578900 \\
\hline $\mathrm{H}$ & 1.17351300 & 3.35851400 & 1.42862300 \\
\hline C & 0.55429600 & 3.84403300 & -0.60908700 \\
\hline C & -0.75764100 & 4.57437100 & -0.31972600 \\
\hline C & 1.54776700 & 4.97079700 & -0.88942500 \\
\hline $\mathrm{H}$ & 0.42052200 & 3.27589400 & -1.53285500 \\
\hline C & -0.75685200 & 5.71931500 & -1.35559000 \\
\hline H & -0.74369500 & 4.96973800 & 0.70553800 \\
\hline $\mathrm{H}$ & -1.62732900 & 3.91798900 & -0.42027400 \\
\hline C & 0.72401600 & 5.90305900 & -1.79377400 \\
\hline $\mathrm{H}$ & 2.45980700 & 4.61814200 & -1.38011700 \\
\hline H & 1.82416900 & 5.46934200 & 0.05070100 \\
\hline $\mathrm{H}$ & -1.37096800 & 5.44007800 & -2.21562800 \\
\hline H & -1.17883600 & 6.63613000 & -0.93298100 \\
\hline $\mathrm{H}$ & 0.84744600 & 5.58437400 & -2.83331500 \\
\hline H & 1.05826900 & 6.94234800 & -1.72502300 \\
\hline C & 1.08630800 & -2.77718100 & 0.37320800 \\
\hline $\mathrm{H}$ & 1.68528100 & -1.93668400 & 0.04191200 \\
\hline C & 0.94152700 & -3.89774100 & -0.60339700 \\
\hline H & 1.16377000 & -3.54866200 & -1.61330500 \\
\hline $\mathrm{H}$ & 1.62810900 & -4.72348300 & -0.36393600 \\
\hline $\mathrm{H}$ & -0.07287700 & -4.31101100 & -0.61655500 \\
\hline C & 0.55460300 & -2.77866800 & 1.66815100 \\
\hline C & 0.78148800 & -1.67558100 & 2.60125900 \\
\hline C & -0.23524100 & -3.86036400 & 2.12412900 \\
\hline C & 1.56916500 & -0.54736200 & 2.27564600 \\
\hline C & 0.18352900 & -1.71925900 & 3.89238500 \\
\hline C & -0.80725300 & -3.88169400 & 3.39832400 \\
\hline $\mathrm{H}$ & -0.40578600 & -4.70297700 & 1.46296600 \\
\hline C & 1.73764600 & 0.49425700 & 3.16289300 \\
\hline H & 2.04809800 & -0.49136000 & 1.30500000 \\
\hline & 0.37370800 & -0.63482300 & 4.78601800 \\
\hline
\end{tabular}




$\begin{array}{lrrr}C & -0.61413200 & -2.83588400 & 4.27398900 \\ H & -1.41151600 & -4.73397400 & 3.69535300 \\ C & 1.12890500 & 0.45614900 & 4.43093300 \\ H & 2.33597500 & 1.35547400 & 2.87858100 \\ H & -0.09647100 & -0.68189400 & 5.76510000 \\ H & -1.06146100 & -2.84844900 & 5.26397100 \\ H & 1.25906200 & 1.28331700 & 5.12186200 \\ \text { N } & 0.78336500 & 1.54371100 & -3.66232200 \\ \text { S } & -0.60985000 & 2.32907200 & -4.05087700 \\ \text { S } & 2.25988900 & 1.97750600 & -4.23624300 \\ 0 & -0.35783600 & 3.50694200 & -4.88014000 \\ 0 & -1.38363300 & 2.49372100 & -2.82279000 \\ 0 & 3.10946000 & 0.80301400 & -4.00965600 \\ 0 & 2.71988400 & 3.24915300 & -3.68195300 \\ C & -1.46782000 & 1.11532500 & -5.04106400 \\ H & -0.92532900 & 0.98212200 & -5.97877100 \\ H & -1.49598200 & 0.17829600 & -4.47967600 \\ H & -2.47380200 & 1.49576200 & -5.23132500 \\ C & 2.10480800 & 2.16476200 & -6.00600000 \\ H & 1.73623700 & 1.22455900 & -6.42002300 \\ H & 1.43174200 & 2.99366100 & -6.22169900 \\ H & 3.11168900 & 2.36995000 & -6.37506800\end{array}$

IM2

\begin{tabular}{lrrr} 
E $=-4138.712968$ & \multicolumn{2}{c}{$G=-4138.200802$} \\
$O$ & 3.30725600 & 2.79089200 & 0.50105800 \\
$O$ & -0.88771600 & 1.80973500 & 2.01327400 \\
$\mathrm{~N}$ & 2.24479900 & 1.29938300 & -0.79785500 \\
$\mathrm{~N}$ & -0.49896600 & 0.79400900 & 0.05727700 \\
$\mathrm{C}$ & 3.65818200 & 1.15584900 & -1.16506200 \\
$\mathrm{C}$ & 4.24866300 & -0.12872000 & -0.63793800 \\
$\mathrm{C}$ & 3.88894200 & -1.42679800 & -0.98419400 \\
$\mathrm{C}$ & 4.53440200 & -2.48307100 & -0.34541000 \\
$\mathrm{C}$ & 5.51918700 & -2.23967700 & 0.61452900 \\
$\mathrm{C}$ & 5.87649100 & -0.93557500 & 0.95042100 \\
$\mathrm{C}$ & 5.23182500 & 0.12188200 & 0.31640200 \\
$\mathrm{C}$ & 5.46903300 & 1.60429700 & 0.49548300 \\
$\mathrm{C}$ & 4.36880700 & 2.27020900 & -0.34439700 \\
$\mathrm{C}$ & 2.17510500 & 2.18894900 & 0.10770800 \\
$\mathrm{C}$ & -0.14903900 & 1.68763300 & 0.90524100 \\
$\mathrm{C}$ & -1.96251300 & 0.84210300 & 1.93236100 \\
$\mathrm{C}$ & -3.32770400 & 1.54735000 & 1.93234500 \\
$\mathrm{C}$ & -3.76452900 & 1.51410100 & 0.48607600 \\
$\mathrm{C}$ & -4.85649200 & 2.14746700 & -0.09936000 \\
$\mathrm{C}$ & -5.08691000 & 1.97212300 & -1.46226600 \\
$\mathrm{C}$ & -4.23984600 & 1.17109000 & -2.23070100 \\
$\mathrm{C}$ & -3.14620600 & 0.53628800 & -1.64664800 \\
$\mathrm{C}$ & -2.92065500 & 0.71936000 & -0.28643500 \\
$\mathrm{C}$ & -1.75820300 & 0.20469100 & 0.52899100 \\
$\mathrm{H}$ & 3.74795700 & 1.25415100 & -2.24965800 \\
$\mathrm{H}$ & 3.12245100 & -1.60640500 & -1.73355900 \\
$\mathrm{H}$ & 4.27071700 & -3.50548800 & -0.59955500 \\
$\mathrm{H}$ & 6.01379700 & -3.07488600 & 1.10240800 \\
$\mathrm{H}$ & 6.64622800 & -0.75059300 & 1.69532600 \\
$\mathrm{H}$ & 5.40722800 & 1.93036500 & 1.53892000 \\
$\mathrm{H}$ & 6.45855400 & 1.89497000 & 0.12358900 \\
$\mathrm{H}$ & 4.72552300 & 3.11064400 & -0.94193500 \\
$\mathrm{H}$ & -1.82777300 & 0.15353200 & 2.76749300 \\
$\mathrm{H}$ & -4.03631000 & 1.00960800 & 2.57292500 \\
$\mathrm{H}$ & -3.23259400 & 2.56206600 & 2.33139900 \\
$\mathrm{H}$ & -5.51842100 & 2.77255000 & 0.49362900 \\
& & & \\
& & &
\end{tabular}




$\begin{array}{lrrr}\text { H } & -5.93368300 & 2.46392100 & -1.93160700 \\ \mathrm{H} & -4.43246300 & 1.04530300 & -3.29190100 \\ \mathrm{H} & -2.46898100 & -0.06853700 & -2.24440200 \\ \mathrm{H} & -1.67804600 & -0.88508900 & 0.53645800 \\ \mathrm{C} & 0.90890100 & 2.73108800 & 0.70810500 \\ \mathrm{Cu} & 0.42110700 & 0.60531400 & -1.67621800 \\ \mathrm{H} & 1.13750600 & 3.18632100 & 1.67596100 \\ \mathrm{C} & 0.32282900 & 3.80468100 & -0.24975600 \\ \mathrm{C} & -0.98908300 & 4.44228900 & 0.23177800 \\ \mathrm{C} & 1.23316800 & 5.00320800 & -0.52067900 \\ \mathrm{H} & 0.15186000 & 3.30929500 & -1.21267900 \\ \mathrm{C} & -1.10181700 & 5.75395300 & -0.58372600 \\ \mathrm{H} & -0.92182300 & 4.65821100 & 1.30583000 \\ \mathrm{H} & -1.85103600 & 3.78094900 & 0.08836100 \\ \mathrm{C} & 0.28427600 & 5.96494300 & -1.24642100 \\ \mathrm{H} & 2.10308900 & 4.73945500 & -1.12710300 \\ \mathrm{H} & 1.58754600 & 5.43108600 & 0.42726000 \\ \mathrm{H} & -1.89041000 & 5.68276200 & -1.33906000 \\ \mathrm{H} & -1.36498500 & 6.59140500 & 0.06956000 \\ \mathrm{H} & 0.24771300 & 5.67896400 & -2.30278200 \\ \mathrm{H} & 0.62055500 & 7.00446600 & -1.19796700 \\ \mathrm{~N} & 0.43537100 & 0.71374400 & -3.64510400 \\ \mathrm{~S} & 0.46231000 & 2.25606900 & -4.24459800 \\ \mathrm{~S} & 1.53950700 & -0.39394700 & -4.17040800 \\ \mathrm{O} & 0.75258600 & 2.25730600 & -5.67775700 \\ \mathrm{O} & 1.22421100 & 3.16861200 & -3.38751000 \\ \mathrm{O} & 1.31252700 & -1.55501200 & -3.30164200 \\ \mathrm{O} & 2.89630400 & 0.14313800 & -4.25232200 \\ \mathrm{O} & -1.26162300 & 2.68225400 & -4.02458900 \\ \mathrm{C} & -1.87031800 & 2.02245400 & -4.64347800 \\ \mathrm{H} & -1.53373900 & 2.57547700 & -2.97275000 \\ \mathrm{H} & -1.37039100 & 3.72122000 & -4.34376600 \\ \mathrm{H} & 1.02643600 & -0.83618400 & -5.82098500 \\ \mathrm{C} & 0.01565400 & -1.24266500 & -5.76824600 \\ \mathrm{H} & 1.06005300 & 0.06323800 & -6.43699100 \\ \mathrm{H} & 1.72813700 & -1.58995300 & -6.18335100 \\ \mathrm{H} & & & \\ & & & \end{array}$

IM3+int1

\begin{tabular}{lrrr}
$E=-5087.950221$ & \multicolumn{2}{c}{$G=-5087.145583$} \\
$O$ & -4.95799500 & 2.10615200 & -0.73925800 \\
$O$ & -2.22897900 & 3.38844600 & -4.10368100 \\
$N$ & -3.57157000 & 0.52025900 & -1.48587300 \\
$N$ & -1.61908600 & 1.39068100 & -3.31694800 \\
$C$ & -4.66224400 & -0.23904100 & -0.85494700 \\
$C$ & -5.49411000 & -0.99100300 & -1.86331500 \\
$C$ & -5.11095500 & -2.09079800 & -2.62221100 \\
$C$ & -6.02966400 & -2.62414700 & -3.52225700 \\
$C$ & -7.30075700 & -2.06245000 & -3.65862900 \\
$C$ & -7.68013700 & -0.96455700 & -2.88677200 \\
$C$ & -6.76690600 & -0.43548700 & -1.97952900 \\
$C$ & -6.97334200 & 0.70153400 & -1.00479700 \\
$C$ & -5.60601000 & 0.87466700 & -0.31928600 \\
$C$ & -3.84350000 & 1.76248200 & -1.38639900 \\
$C$ & -2.27762200 & 2.47797300 & -3.14184000 \\
$C$ & -1.53202800 & 2.77318300 & -5.22867400 \\
$C$ & -0.44555800 & 3.71456600 & -5.73881700 \\
$C$ & 0.74319300 & 3.36135600 & -4.87437800 \\
$C$ & 1.91019500 & 4.08616000 & -4.66180500 \\
$C$ & 2.85870500 & 3.59022300 & -3.76839200 \\
$C$ & 2.63587500 & 2.39201200 & -3.08872700 \\
$C$ & 1.46619500 & 1.66245700 & -3.29868700
\end{tabular}




\begin{tabular}{|c|c|c|c|}
\hline & 0.53600800 & 2.15099000 & -4.21034400 \\
\hline & -0.83228400 & 1.58691000 & -4.54094800 \\
\hline H & -4.23006600 & -0.89007500 & -0.09252600 \\
\hline & -4.12291700 & -2.52644700 & -2.51621400 \\
\hline & -5.74149100 & -3.48176600 & -4.12212300 \\
\hline$n$ & -8.00394600 & -2.48531800 & -4.37043100 \\
\hline & -8.67385000 & -0.53672500 & -2.98899600 \\
\hline & -7.28231000 & 1.63376800 & -1.48962300 \\
\hline H & -7.74443200 & 0.45570600 & -0.26599800 \\
\hline H & -5.67346100 & 0.92203100 & 0.76802000 \\
\hline & -2.29224800 & 2.50455700 & -5.96292400 \\
\hline H & -0.25383700 & 3.51176700 & -6.79965500 \\
\hline H & -0.75592700 & 4.76039700 & -5.65633300 \\
\hline & 2.07493800 & 5.03287000 & -5.16930100 \\
\hline t & 3.77178200 & 4.15001100 & -3.58811800 \\
\hline H & 3.37280100 & 2.02678300 & -2.37985700 \\
\hline $\boldsymbol{H}$ & 1.28219700 & 0.74642500 & -2.74165600 \\
\hline & -0.81671600 & 0.66662700 & -5.12294500 \\
\hline c & -2.97617200 & 2.87508200 & -1.88125000 \\
\hline $\mathrm{Cu}$ & -1.85484800 & -0.33541000 & -2.29190200 \\
\hline C & -1.96654800 & -1.79399200 & -1.07549200 \\
\hline c & -2.16388100 & -2.85025300 & -0.48563100 \\
\hline Si & -2.38500900 & -4.60579000 & -0.01838600 \\
\hline c & -3.02885200 & -4.74799100 & 1.75102100 \\
\hline H & -2.32670800 & -4.29973300 & 2.46287000 \\
\hline-1 & -3.99324200 & -4.23913200 & 1.86272000 \\
\hline $\mathbf{H}$ & -3.16912000 & -5.79865900 & 2.03251300 \\
\hline . & -3.64397300 & -5.33525500 & -1.21678100 \\
\hline H & -3.78081700 & -6.41110100 & -1.05374500 \\
\hline $\mathbf{H}$ & -4.61992400 & -4.84724600 & -1.10985000 \\
\hline H & -3.30200700 & -5.17777900 & -2.24557300 \\
\hline$c$ & -0.73234000 & -5.48669600 & -0.18398200 \\
\hline - & -0.31283200 & -5.29792300 & -1.17787700 \\
\hline H & -0.01455200 & -5.11237300 & 0.55435600 \\
\hline $\mathrm{H}$ & -0.83589600 & -6.56947600 & -0.04325800 \\
\hline t & -3.60126100 & 3.74650800 & -2.08893400 \\
\hline c & -1.89981400 & 3.23696800 & -0.82648300 \\
\hline C & -0.93446600 & 4.33893400 & -1.28041900 \\
\hline 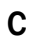 & -2.47369200 & 3.78116500 & 0.49038800 \\
\hline $\mathbf{H}$ & -1.31890500 & 2.32654400 & -0.62530000 \\
\hline c & -0.23344600 & 4.72878200 & 0.02630900 \\
\hline+ & -1.50858300 & 5.18442400 & -1.68374100 \\
\hline $\mathbf{H}$ & -0.23563400 & 4.00885700 & -2.05582300 \\
\hline c & -1.31197300 & 4.58108400 & 1.13032200 \\
\hline t & -2.85040000 & 2.98542700 & 1.13975100 \\
\hline H & -3.32084200 & 4.44241100 & 0.26985500 \\
\hline H & 0.59372100 & 4.03426600 & 0.21038900 \\
\hline H & 0.19379500 & 5.73457600 & -0.01427700 \\
\hline H & -0.90773500 & 4.06286700 & 2.00445700 \\
\hline H & -1.66409500 & 5.55741600 & 1.47624000 \\
\hline $\mathbf{N}$ & -0.73187300 & -1.41861300 & -3.72021100 \\
\hline 5 & 0.66456800 & -2.14442800 & -3.21077400 \\
\hline 5 & -1.85745700 & -2.19026600 & -4.64233000 \\
\hline o & 0.58824700 & -3.59973500 & -3.20831300 \\
\hline & 1.08014000 & -1.41213400 & -2.01818400 \\
\hline & -2.79481700 & -1.10760500 & -4.96359100 \\
\hline & -2.40139900 & -3.41931700 & -4.08250800 \\
\hline & 1.85192400 & -1.69824500 & -4.48384900 \\
\hline & 1.60326600 & -2.19628000 & -5.42123100 \\
\hline & 1.85531100 & -0.61427400 & -4.60767000 \\
\hline & & & \\
\hline
\end{tabular}




$\begin{array}{llll}\text { C } & -1.02184100 & -2.65951600 & -6.15374000 \\ \text { H } & -0.54232000 & -1.78463800 & -6.59615700 \\ \text { H } & -0.30031200 & -3.44560100 & -5.92185300 \\ \text { H } & -1.78929300 & -3.05227600 & -6.82407600 \\ \text { C } & -5.36401100 & 0.19507900 & -5.88126200 \\ \text { H } & -5.71339000 & 0.02469900 & -4.86928500 \\ \text { C } & -5.27837000 & -1.00757400 & -6.76378400 \\ \text { H } & -5.63445800 & -1.89246700 & -6.23313900 \\ \text { H } & -5.87640700 & -0.88618200 & -7.67763400 \\ \text { H } & -4.24154000 & -1.20453200 & -7.06239000 \\ \text { C } & -4.99709500 & 1.48537700 & -6.28617300 \\ \text { C } & -5.18071000 & 2.65687500 & -5.43128600 \\ \text { C } & -4.44157600 & 1.69885800 & -7.56918000 \\ \text { C } & -5.74364900 & 2.57836500 & -4.13633800 \\ \text { C } & -4.80414500 & 3.94141600 & -5.91737800 \\ \text { C } & -4.06837400 & 2.96859100 & -8.01948100 \\ \text { H } & -4.29529800 & 0.84656200 & -8.22399700 \\ \text { C } & -5.94091400 & 3.70770100 & -3.37045300 \\ \text { H } & -6.04538900 & 1.61528900 & -3.73698800 \\ \text { C } & -4.99923000 & 5.08195600 & -5.10084300 \\ \text { C } & -4.23804500 & 4.07701300 & -7.21744000 \\ \text { H } & -3.64302200 & 3.07747400 & -9.01335600 \\ \text { C } & -5.56216200 & 4.97462400 & -3.85314600 \\ \text { H } & -6.37400400 & 3.61849300 & -2.37936400 \\ \text { H } & -4.70145000 & 6.05342800 & -5.48756200 \\ \text { H } & -3.95553200 & 5.06626500 & -7.56713200 \\ \text { H } & -5.71893100 & 5.85898900 & -3.24240600 \\ & & & \end{array}$

TS1R

\begin{tabular}{lrrr} 
E $=-5087.962625$ & \multicolumn{2}{c}{$G=-5087.149309$} \\
$\mathrm{O}$ & 3.29922700 & 2.80866800 & 0.53823200 \\
$\mathrm{O}$ & -0.73231100 & 2.01520500 & 2.04835300 \\
$\mathrm{~N}$ & 2.39763400 & 1.12611900 & -0.64738400 \\
$\mathrm{~N}$ & -0.50367100 & 0.86695400 & 0.13304100 \\
$\mathrm{C}$ & 3.86883100 & 1.03951800 & -0.89857800 \\
$\mathrm{C}$ & 4.53912400 & -0.26301200 & -0.53906100 \\
$\mathrm{C}$ & 4.66105700 & -1.41094500 & -1.31365600 \\
$\mathrm{C}$ & 5.33100800 & -2.50990800 & -0.78038800 \\
$\mathrm{C}$ & 5.88371600 & -2.45155500 & 0.49951800 \\
$\mathrm{C}$ & 5.80405800 & -1.28153600 & 1.25270900 \\
$\mathrm{C}$ & 5.13881900 & -0.18404900 & 0.71927900 \\
$\mathrm{C}$ & 4.99057100 & 1.19526900 & 1.31286500 \\
$\mathrm{C}$ & 4.43853400 & 2.03187800 & 0.13928100 \\
$\mathrm{C}$ & 2.21882300 & 2.15668200 & 0.10022400 \\
$\mathrm{C}$ & -0.11562400 & 1.82461200 & 0.87736000 \\
$\mathrm{C}$ & -1.65508400 & 0.90236100 & 2.20845200 \\
$\mathrm{C}$ & -3.05953600 & 1.40992300 & 2.53593000 \\
$\mathrm{C}$ & -3.73672900 & 1.48195200 & 1.18859000 \\
$\mathrm{C}$ & -4.95241900 & 2.07624400 & 0.86699800 \\
$\mathrm{C}$ & -5.39554100 & 2.03157400 & -0.45289600 \\
$\mathrm{C}$ & -4.62766100 & 1.40650000 & -1.43645100 \\
$\mathrm{C}$ & -3.41209400 & 0.81001200 & -1.11393000 \\
$\mathrm{C}$ & -2.97963200 & 0.84541100 & 0.20618000 \\
$\mathrm{C}$ & -1.69028000 & 0.29570600 & 0.78238700 \\
$\mathrm{H}$ & 4.03831400 & 1.33596500 & -1.93338900 \\
$\mathrm{H}$ & 4.23507900 & -1.43205300 & -2.30995000 \\
$\mathrm{H}$ & 5.43015800 & -3.41642000 & -1.37021500 \\
$\mathrm{H}$ & 6.40160200 & -3.31660900 & 0.90325500 \\
$\mathrm{H}$ & 6.26779200 & -1.22620300 & 2.23377800 \\
$\mathrm{H}$ & 4.27552200 & 1.20941700 & 2.14434100 \\
$\mathrm{H}$ & 5.93620200 & 1.59083400 & 1.69571300
\end{tabular}




\begin{tabular}{|c|c|c|c|}
\hline H & 30900 & 200 & 9000 \\
\hline H & -1.22818500 & 0.25318700 & 2.97407400 \\
\hline H & -3.56521600 & 0.69840600 & 3.20052200 \\
\hline H & -3.01924400 & 2.37159700 & 3.05693500 \\
\hline H & -5.54334800 & 2.57656400 & 1.62971700 \\
\hline H & -6.33896400 & 2.49866300 & -0.72050100 \\
\hline H & -4.97053800 & 1.40132900 & -2.46696900 \\
\hline H & -2.79395700 & 0.34879900 & -1.87317700 \\
\hline H & -1.64616500 & -0.79607700 & 0.75404400 \\
\hline C & 0.93515200 & 2.81495200 & 0.49692900 \\
\hline $\mathrm{Cu}$ & 0.79128600 & -0.01307100 & -1.44719100 \\
\hline C & -0.50854000 & -1.27082000 & -1.92307400 \\
\hline C & -1.50642400 & -1.93752400 & -2.17474800 \\
\hline Si & -3.06620500 & -2.87138900 & -2.35367700 \\
\hline C & -3.99031600 & -2.80748400 & -0.71336200 \\
\hline H & -3.35958800 & -3.17357600 & 0.10473400 \\
\hline H & -4.27938600 & -1.77762100 & -0.47679400 \\
\hline H & -4.89986900 & -3.41926400 & -0.74473700 \\
\hline C & -4.11709100 & -2.09045600 & -3.70926900 \\
\hline H & -5.06106300 & -2.63287300 & -3.83825700 \\
\hline H & -4.36009100 & -1.05212400 & -3.45619000 \\
\hline H & -3.59296600 & -2.09149200 & -4.67166300 \\
\hline C & -2.66996400 & -4.66160800 & -2.79674900 \\
\hline H & -2.09459700 & -4.71966800 & -3.72726100 \\
\hline H & -2.07900000 & -5.14233500 & -2.00813200 \\
\hline H & -3.58746100 & -5.24724900 & -2.92888700 \\
\hline H & 1.14349200 & 3.46212300 & 1.35310200 \\
\hline C & 0.42273500 & 3.65227300 & -0.69726500 \\
\hline C & -0.86663000 & 4.42441500 & -0.40454600 \\
\hline C & 1.38162300 & 4.72027500 & -1.22395400 \\
\hline H & 0.21497400 & 2.94922600 & -1.50475500 \\
\hline C & -0.94981200 & 5.44790400 & -1.55874900 \\
\hline H & -0.78161500 & 4.93351900 & 0.56593400 \\
\hline H & -1.73944000 & 3.76513100 & -0.36531700 \\
\hline C & 0.47525300 & 5.52161900 & -2.17272200 \\
\hline H & 2.24865300 & 4.29562300 & -1.73988100 \\
\hline H & 1.74424600 & 5.34608600 & -0.39595500 \\
\hline H & -1.66615800 & 5.10650200 & -2.30964900 \\
\hline H & -1.28946700 & 6.42297500 & -1.19553300 \\
\hline H & 0.48006000 & 5.04268000 & -3.15704400 \\
\hline H & 0.82521000 & 6.54974900 & -2.30568500 \\
\hline C & 1.45938500 & -2.01436000 & -0.53549100 \\
\hline H & 2.30580400 & -1.37722900 & -0.29942800 \\
\hline c & 1.79005600 & -2.99071600 & -1.63316800 \\
\hline H & 2.19932100 & -2.46224300 & -2.49618200 \\
\hline H & 2.55474600 & -3.68507600 & -1.25583300 \\
\hline H & 0.92766000 & -3.56332000 & -1.97332700 \\
\hline C & 0.67178400 & -2.42614600 & 0.62565700 \\
\hline C & 0.81360600 & -1.78194900 & 1.91011800 \\
\hline C & -0.23295900 & -3.47278400 & 0.51278300 \\
\hline C & 1.70610900 & -0.70423100 & 2.14831700 \\
\hline C & 0.02084100 & -2.23598500 & 3.00525700 \\
\hline C & -1.00824200 & -3.91448800 & 1.59781200 \\
\hline H & -0.37163900 & -3.94981600 & -0.44932400 \\
\hline C & 1.78626200 & -0.09800500 & 3.37726300 \\
\hline H & 2.33364800 & -0.34113300 & 1.34588600 \\
\hline C & 0.14214700 & -1.60451900 & 4.27202900 \\
\hline C & -0.89103500 & -3.30623200 & 2.82141200 \\
\hline H & -1.70446800 & -4.73557600 & 1.45593300 \\
\hline c & 0.99881800 & -0.55110800 & 4.45783700 \\
\hline 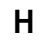 & 2.46316000 & 0.73885200 & 3.52349300 \\
\hline
\end{tabular}




$\begin{array}{lrrr}\mathrm{H} & -0.46743800 & -1.97124600 & 5.09397900 \\ \mathrm{H} & -1.49062400 & -3.63660900 & 3.66550000 \\ \mathrm{H} & 1.07612700 & -0.06789400 & 5.42679100 \\ \mathrm{~N} & 0.68130700 & 1.07667500 & -3.30684400 \\ \mathrm{~S} & -0.59070900 & 1.88153100 & -3.95807700 \\ \mathrm{~S} & 2.15725200 & 1.04339000 & -3.99788200 \\ \mathrm{O} & -0.15225800 & 2.85501200 & -4.96017100 \\ \mathrm{O} & -1.44347500 & 2.33937200 & -2.86742100 \\ \mathrm{O} & 2.77622600 & -0.18742300 & -3.47286700 \\ \mathrm{O} & 2.91081200 & 2.28191100 & -3.80220600 \\ \mathrm{C} & -1.51113200 & 0.60530600 & -4.80780800 \\ \mathrm{H} & -0.93496200 & 0.25705600 & -5.66673800 \\ \mathrm{H} & -1.68198000 & -0.21280100 & -4.10515700 \\ \mathrm{H} & -2.45267300 & 1.04799600 & -5.14025600 \\ \mathrm{C} & 1.97146100 & 0.79856700 & -5.75927500 \\ \mathrm{H} & 1.44518600 & -0.14313600 & -5.92466600 \\ \mathrm{H} & 1.43435600 & 1.64674900 & -6.18359700 \\ \mathrm{H} & 2.98450200 & 0.73888100 & -6.16174100\end{array}$

TS1S

\begin{tabular}{lrrr} 
E $=-5087.959851$ & \multicolumn{2}{c}{$G=-5087.145293$} \\
$O$ & -2.01836400 & 1.40449700 & 2.04954700 \\
$O$ & 0.66793300 & 3.09917700 & -0.84559400 \\
$\mathrm{~N}$ & -0.78715200 & -0.35075200 & 1.42154100 \\
$\mathrm{~N}$ & 1.29342500 & 0.99998900 & -0.36869300 \\
$\mathrm{C}$ & -1.28031000 & -0.71139800 & 2.77802800 \\
$\mathrm{C}$ & -2.14325800 & -1.94385800 & 2.85422600 \\
$\mathrm{C}$ & -1.72885100 & -3.26814500 & 2.94728300 \\
$\mathrm{C}$ & -2.69789100 & -4.26593800 & 3.03867800 \\
$\mathrm{C}$ & -4.05394000 & -3.93678400 & 3.06236800 \\
$\mathrm{C}$ & -4.46048700 & -2.60363100 & 3.03170100 \\
$\mathrm{C}$ & -3.49408300 & -1.60764000 & 2.94051300 \\
$\mathrm{C}$ & -3.68954300 & -0.11172000 & 3.00294300 \\
$\mathrm{C}$ & -2.25370500 & 0.44747000 & 3.09984200 \\
$\mathrm{C}$ & -1.25466400 & 0.81605500 & 1.14052800 \\
$\mathrm{C}$ & 0.36211000 & 1.86361700 & -0.43276100 \\
$\mathrm{C}$ & 2.08986800 & 3.12223200 & -1.06176900 \\
$\mathrm{C}$ & 2.80806300 & 3.99776400 & -0.02251600 \\
$\mathrm{C}$ & 3.89564100 & 3.10192400 & 0.50879200 \\
$\mathrm{C}$ & 4.99281200 & 3.46699500 & 1.28105600 \\
$\mathrm{C}$ & 5.90051600 & 2.48666700 & 1.67755800 \\
$\mathrm{C}$ & 5.69484900 & 1.15422200 & 1.31758500 \\
$\mathrm{C}$ & 4.59110600 & 0.79088100 & 0.54762500 \\
$\mathrm{C}$ & 3.69889500 & 1.77326800 & 0.13482400 \\
$\mathrm{C}$ & 2.53014100 & 1.64713000 & -0.82384200 \\
$\mathrm{H}$ & -0.43572500 & -0.75491700 & 3.46117400 \\
$\mathrm{H}$ & -0.66803700 & -3.50141300 & 2.96258400 \\
$\mathrm{H}$ & -2.39373600 & -5.30612400 & 3.10734400 \\
$\mathrm{H}$ & -4.79835200 & -4.72427800 & 3.13763100 \\
$\mathrm{H}$ & -5.51487000 & -2.34907300 & 3.10157800 \\
$\mathrm{H}$ & -4.18089000 & 0.28476800 & 2.10665800 \\
$\mathrm{H}$ & -4.30010500 & 0.18321200 & 3.86228400 \\
$\mathrm{H}$ & -2.02770000 & 0.94519400 & 4.04076000 \\
$\mathrm{H}$ & 2.24753300 & 3.46668200 & -2.08526300 \\
$\mathrm{H}$ & 3.19002000 & 4.92856700 & -0.45324100 \\
$\mathrm{H}$ & 2.10379300 & 4.25215300 & 0.77690900 \\
$\mathrm{H}$ & 5.14543700 & 4.50467700 & 1.56548900 \\
$\mathrm{H}$ & 6.76782100 & 2.76065600 & 2.27134400 \\
$\mathrm{H}$ & 6.40228400 & 0.39257400 & 1.63511900 \\
$\mathrm{H}$ & 4.41785300 & -0.24181000 & 0.27511000 \\
$\mathrm{H}$ & 2.84563900 & 1.13377900 & -1.73955000 \\
& & & \\
& &
\end{tabular}




\begin{tabular}{|c|c|c|c|}
\hline C & -1.09363800 & 0200 & -0.16347700 \\
\hline $\mathrm{Cu}$ & 0.95926900 & -0.98218500 & 0.62246900 \\
\hline C & 2.45233300 & -1.78156700 & -0.20314800 \\
\hline C & 3.43727900 & -2.16739100 & -0.82537300 \\
\hline Si & 4.98546400 & -2.48475600 & -1.74477600 \\
\hline C & 5.23793700 & -1.02810000 & -2.91670400 \\
\hline $\mathrm{H}$ & 4.39967800 & -0.94532500 & -3.61870900 \\
\hline $\mathbf{H}$ & 5.29964500 & -0.09170800 & -2.34952300 \\
\hline $\mathrm{H}$ & 6.15926100 & -1.13143200 & -3.50170000 \\
\hline C & 6.41749600 & -2.57353400 & -0.52261100 \\
\hline $\mathrm{H}$ & 7.36345400 & -2.78245600 & -1.03585000 \\
\hline $\mathbf{H}$ & 6.53086000 & -1.62377900 & 0.01264300 \\
\hline H & 6.25440800 & -3.36254200 & 0.21990400 \\
\hline C & 4.85551300 & -4.09348100 & -2.71330700 \\
\hline $\mathbf{H}$ & 4.68912200 & -4.94232300 & -2.04110500 \\
\hline $\mathrm{H}$ & 4.02074400 & -4.05914700 & -3.42219500 \\
\hline $\mathrm{H}$ & 5.77316200 & -4.28650200 & -3.28137500 \\
\hline $\mathrm{H}$ & -1.36779900 & 0.83784300 & -0.93466300 \\
\hline C & -2.06854100 & 2.74941800 & -0.30667300 \\
\hline C & -2.01722600 & 3.43165100 & -1.69288100 \\
\hline C & -3.55645500 & 2.30098400 & -0.14931300 \\
\hline $\mathrm{H}$ & -1.83151600 & 3.49066400 & 0.46300400 \\
\hline C & -3.42623000 & 3.99367700 & -1.88591500 \\
\hline $\mathrm{H}$ & -1.80644900 & 2.68479600 & -2.47047500 \\
\hline $\mathrm{H}$ & -1.23893100 & 4.19212800 & -1.75283700 \\
\hline C & -4.32290100 & 2.87147000 & -1.35827700 \\
\hline $\mathrm{H}$ & -3.96100200 & 2.67685700 & 0.79351000 \\
\hline H & -3.64305900 & 1.20912100 & -0.11645900 \\
\hline H & -3.55314600 & 4.89746100 & -1.27577200 \\
\hline $\mathrm{H}$ & -3.63883000 & 4.26545800 & -2.92484400 \\
\hline $\mathrm{H}$ & -5.33039100 & 3.20874200 & -1.09626200 \\
\hline H & -4.42803400 & 2.09979500 & -2.13015200 \\
\hline C & 0.05915300 & -2.44744600 & -0.89883700 \\
\hline $\mathrm{H}$ & 0.89751500 & -2.64522700 & -1.55096000 \\
\hline C & -0.26957800 & -3.62864400 & -0.03561100 \\
\hline H & -0.63662900 & -4.43756900 & -0.68550700 \\
\hline $\mathrm{H}$ & 0.62392500 & -3.98146400 & 0.48328300 \\
\hline $\mathrm{H}$ & -1.03707800 & -3.42383500 & 0.70754400 \\
\hline C & -0.97360200 & -1.63714300 & -1.52477300 \\
\hline C & -0.64882800 & -0.78705900 & -2.64767400 \\
\hline C & -2.29580500 & -1.71618500 & -1.10172900 \\
\hline C & 0.68545700 & -0.54557800 & -3.06886300 \\
\hline C & -1.70303200 & -0.12407500 & -3.33767300 \\
\hline C & -3.32711800 & -1.05275300 & -1.78989800 \\
\hline H & -2.54636300 & -2.31341700 & 50800 \\
\hline C & 0.95064900 & 0.29701500 & -4.12060100 \\
\hline H & 1.51362200 & -0.99305200 & -2.52923200 \\
\hline C & -1.39736300 & 0.72672000 & -4.43209200 \\
\hline C & -3.04249700 & -0.29047600 & -2.89664500 \\
\hline H & -4.35147100 & -1.15708700 & -1.44555000 \\
\hline C & -0.09847500 & 0.93742600 & -4.81614000 \\
\hline H & 1.98058300 & 0.47556300 & -4.41673300 \\
\hline $\mathrm{H}$ & -2.21466600 & 1.22194500 & -4.95018900 \\
\hline H & -3.83849200 & 0.20820400 & -3.44312000 \\
\hline H & 0.12640200 & 1.59810800 & -5.64777900 \\
\hline $\mathbf{N}$ & 1.92532500 & -0.32484600 & 2.49686600 \\
\hline $\mathbf{S}$ & 1.66397900 & 1.10309700 & 3.28119500 \\
\hline $\mathbf{S}$ & 2.33782200 & -1.67296100 & 3.33856200 \\
\hline 0 & 0.66990000 & 0.99477900 & 4.35021800 \\
\hline 0 & 1.39128000 & 2.10320200 & 2.2470730 \\
\hline 0 & 1.52020300 & -2.77269600 & 2.8081330 \\
\hline
\end{tabular}




$\begin{array}{lrrr}\text { O } & 2.35809900 & -1.46086300 & 4.78487300 \\ \text { C } & 3.21643100 & 1.52620700 & 4.05037000 \\ \text { H } & 3.44389200 & 0.76511700 & 4.79860000 \\ \text { H } & 3.98394600 & 1.58232900 & 3.27811000 \\ \text { H } & 3.07880200 & 2.49907900 & 4.52651700 \\ \text { C } & 4.02156200 & -1.98861600 & 2.83180200 \\ \text { H } & 4.03541600 & -2.14792400 & 1.75312400 \\ \text { H } & 4.63137400 & -1.12611900 & 3.10770900 \\ \text { H } & 4.35600500 & -2.88206500 & 3.36372300\end{array}$

TS1S'

\begin{tabular}{lrrr} 
E $=-5087.946426$ & \multicolumn{2}{c}{$\mathrm{G}=-5087.132558$} \\
$\mathrm{O}$ & -2.07605000 & 1.60077400 & 2.06858900 \\
$\mathrm{O}$ & 0.67812900 & 3.40561100 & -0.67340700 \\
$\mathrm{~N}$ & -0.70793300 & -0.06735900 & 1.49744800 \\
$\mathrm{~N}$ & 1.33696900 & 1.30011800 & -0.27620000 \\
$\mathrm{C}$ & -1.26708500 & -0.49424900 & 2.80319700 \\
$\mathrm{C}$ & -2.13806500 & -1.72120700 & 2.70600000 \\
$\mathrm{C}$ & -1.73611600 & -3.04756500 & 2.58679200 \\
$\mathrm{C}$ & -2.71003800 & -4.03708400 & 2.47584100 \\
$\mathrm{C}$ & -4.06442400 & -3.70191000 & 2.51690700 \\
$\mathrm{C}$ & -4.46145500 & -2.37908600 & 2.70404300 \\
$\mathrm{C}$ & -3.48831300 & -1.39016500 & 2.80677800 \\
$\mathrm{C}$ & -3.68053900 & 0.07701100 & 3.11380200 \\
$\mathrm{C}$ & -2.25114200 & 0.65288600 & 3.14931800 \\
$\mathrm{C}$ & -1.23612700 & 1.06481000 & 1.19930700 \\
$\mathrm{C}$ & 0.38985900 & 2.14865400 & -0.32908600 \\
$\mathrm{C}$ & 2.10810800 & 3.46670700 & -0.85568200 \\
$\mathrm{C}$ & 2.78882700 & 4.32497000 & 0.22136400 \\
$\mathrm{C}$ & 3.88005600 & 3.43093300 & 0.75017300 \\
$\mathrm{C}$ & 4.96216500 & 3.79159900 & 1.54600100 \\
$\mathrm{C}$ & 5.86812600 & 2.80944700 & 1.94224800 \\
$\mathrm{C}$ & 5.67778600 & 1.48106200 & 1.55818800 \\
$\mathrm{C}$ & 4.59578700 & 1.12292800 & 0.75669600 \\
$\mathrm{C}$ & 3.70663800 & 2.10835500 & 0.34532000 \\
$\mathrm{C}$ & 2.57604200 & 1.99349100 & -0.65699000 \\
$\mathrm{H}$ & -0.45911600 & -0.60599500 & 3.52163700 \\
$\mathrm{H}$ & -0.68198500 & -3.30284300 & 2.62689800 \\
$\mathrm{H}$ & -2.41164700 & -5.07632200 & 2.37584600 \\
$\mathrm{H}$ & -4.81533300 & -4.48214900 & 2.43282500 \\
$\mathrm{H}$ & -5.51619800 & -2.12901300 & 2.78358100 \\
$\mathrm{H}$ & -4.27916200 & 0.60011800 & 2.35986500 \\
$\mathrm{H}$ & -4.18451800 & 0.21411800 & 4.07655400 \\
$\mathrm{H}$ & -1.99149600 & 1.17605000 & 4.06706100 \\
$\mathrm{H}$ & 2.27732300 & 3.84732200 & -1.86441500 \\
$\mathrm{H}$ & 3.16351800 & 5.27253800 & -0.17781400 \\
$\mathrm{H}$ & 2.06505300 & 4.54474800 & 1.01337400 \\
$\mathrm{H}$ & 5.10200100 & 4.82488500 & 1.85207500 \\
$\mathrm{H}$ & 6.72224700 & 3.07847300 & 2.55695600 \\
$\mathrm{H}$ & 6.38708000 & 0.72100800 & 1.87503300 \\
$\mathrm{H}$ & 4.44176300 & 0.09631100 & 0.44798000 \\
$\mathrm{H}$ & 2.93988300 & 1.52105400 & -1.57525900 \\
$\mathrm{C}$ & -1.06333500 & 1.79329800 & -0.11550100 \\
$\mathrm{Cu}$ & 1.03151300 & -0.66002100 & 0.66496800 \\
$\mathrm{C}$ & 2.45143900 & -1.42296900 & -0.25166100 \\
$\mathrm{C}$ & 3.47120800 & -1.80697500 & -0.81174500 \\
$\mathrm{Si}$ & 5.02181200 & -2.52634600 & -1.46542100 \\
$\mathrm{C}$ & 4.72468900 & -3.21926900 & -3.18978200 \\
$\mathrm{H}$ & 3.96553700 & -4.00759200 & -3.16138300 \\
$\mathrm{H}$ & 4.37336500 & -2.43817900 & -3.87323600 \\
$\mathrm{H}$ & 5.64707800 & -3.64320000 & -3.60471900 \\
& & & \\
\hline
\end{tabular}




\begin{tabular}{|c|c|c|c|}
\hline & 6.35087300 & -1.19010600 & 0600 \\
\hline & 7.27765100 & -1.58843600 & -1.97238500 \\
\hline H & 6.02708500 & -0.34545100 & -2.16072800 \\
\hline H & 6.58495800 & -0.79702500 & -0.54679300 \\
\hline C & 5.55288800 & -3.89682500 & -0.28851300 \\
\hline H & 5.73896600 & -3.49900300 & 0.71576400 \\
\hline H & 4.77126300 & -4.65953400 & -0.20279500 \\
\hline H & 6.47249400 & -4.38453500 & -0.63351200 \\
\hline H & -1.26958700 & 1.03473200 & -0.88273200 \\
\hline C & -2.08546600 & 2.92274400 & -0.32287800 \\
\hline C & -1.98232100 & 3.60039900 & -1.70959000 \\
\hline C & -3.55314900 & 2.38944100 & -0.25265800 \\
\hline H & -1.93236500 & 3.67490400 & 0.45656900 \\
\hline C & -3.41664400 & 4.02086200 & -2.03552600 \\
\hline H & -1.63761400 & 2.87259100 & -2.45886100 \\
\hline H & -1.27367100 & 4.42888000 & -1.71397500 \\
\hline C & -4.23901900 & 2.82069700 & -1.56265600 \\
\hline H & -4.05933500 & 2.81772900 & 0.61638300 \\
\hline H & -3.59014600 & 1.30114100 & -0.12535200 \\
\hline H & -3.68418200 & 4.91390600 & -1.45600900 \\
\hline H & -3.56184900 & 4.25746100 & -3.09404500 \\
\hline H & -5.30065300 & 3.04673500 & -1.42569000 \\
\hline H & -4.17061000 & 2.01793500 & -2.30761200 \\
\hline C & 0.07843800 & -2.45010600 & -0.34068200 \\
\hline H & -0.24139300 & -2.66838900 & 0.67373100 \\
\hline C & -0.95085400 & -1.70169600 & -1.15330600 \\
\hline H & -1.58526300 & -2.41987000 & -1.68831400 \\
\hline H & -1.59507300 & -1.11662200 & -0.49799000 \\
\hline H & -0.49488800 & -1.04132600 & -1.89943400 \\
\hline C & 0.78915200 & -3.56099700 & -0.98361400 \\
\hline c & 1.50733400 & -4.53903300 & -0.20858100 \\
\hline C & 0.71647700 & -3.72737600 & -2.35613100 \\
\hline C & 1.72185900 & -4.40058700 & 1.18306200 \\
\hline C & 2.06031100 & -5.67455400 & -0.86389000 \\
\hline c & 1.27445600 & -4.84744800 & -3.00057000 \\
\hline H & 0.21307300 & -2.98451200 & -2.96483100 \\
\hline C & 2.41563800 & -5.34983600 & 1.89266000 \\
\hline H & 1.38043000 & -3.52013200 & 1.71300200 \\
\hline C & 2.76111100 & -6.64737100 & -0.10394100 \\
\hline C & 1.92180100 & -5.80990700 & -2.26998500 \\
\hline H & 1.18368500 & -4.94331500 & -4.07811600 \\
\hline c & 2.93416000 & -6.49362800 & 1.24812100 \\
\hline H & 2.56644100 & -5.20448700 & 2.95818900 \\
\hline H & 3.16934800 & -7.51373300 & -0.61830600 \\
\hline H & 2.34747800 & -6.68277900 & -2.75839700 \\
\hline r & 3.47792400 & -7.24007500 & 1.81920300 \\
\hline $\mathbf{N}$ & 2.00537500 & 0.07088200 & 2.66088800 \\
\hline S & 1.57763800 & 1.47832700 & 3.39855800 \\
\hline $\mathbf{S}$ & 2.39603900 & -1.21573500 & 3.59922900 \\
\hline 0 & 0.56292900 & 1.28794200 & 4.43937000 \\
\hline 0 & 1.23511600 & 2.42680400 & 2.33309200 \\
\hline 0 & 1.37862900 & -2.26687000 & 3.42157700 \\
\hline 0 & 2.73740200 & -0.84879000 & 4.97420100 \\
\hline c & 3.04550300 & 2.09845100 & 4.19755500 \\
\hline H & 3.33224200 & 1.39187700 & 4.97631900 \\
\hline H & 3.82681100 & 2.20646500 & 3.44583900 \\
\hline H & 2.78358700 & 3.06852900 & 4.62507300 \\
\hline C & 3.89091500 & -1.81443400 & 2.83188400 \\
\hline & 3.69529100 & -2.05964100 & 1.78731100 \\
\hline & 4.64576000 & -1.03034400 & 2.91062500 \\
\hline H & 4.19531000 & -2.70270100 & 3.39014200 \\
\hline
\end{tabular}




$\begin{array}{lrrr}3 a & & & \\ \text { E }=-949.266655 & G=-948.993317 \\ \mathrm{C} & 1.21389700 & -0.76134500 & -0.08950400 \\ \mathrm{C} & 2.38112500 & -0.45039800 & 0.03869800 \\ \mathrm{Si} & 4.13376200 & 0.05975700 & 0.24676600 \\ \mathrm{C} & 4.24178100 & 1.92287000 & 0.00093900 \\ \mathrm{H} & 3.90481700 & 2.20917700 & -1.00139100 \\ \mathrm{H} & 3.61896200 & 2.45298000 & 0.72966100 \\ \mathrm{H} & 5.27401900 & 2.27221500 & 0.12178100 \\ \mathrm{C} & 4.70409000 & -0.39905600 & 1.97997500 \\ \mathrm{H} & 5.74745200 & -0.10034600 & 2.13620800 \\ \mathrm{H} & 4.09290700 & 0.09866300 & 2.74048900 \\ \mathrm{H} & 4.63411100 & -1.47932100 & 2.14689700 \\ \mathrm{C} & 5.18532900 & -0.82492200 & -1.03883500 \\ \mathrm{H} & 5.12239300 & -1.91216700 & -0.92174300 \\ \mathrm{H} & 4.85920100 & -0.57689600 & -2.05466200 \\ \mathrm{H} & 6.23859900 & -0.53583100 & -0.94261900 \\ \mathrm{C} & -5.28613900 & -0.52716200 & 0.64234900 \\ \mathrm{C} & -4.79971200 & 0.72040400 & 0.35232800 \\ \mathrm{C} & -3.42523900 & 0.92255200 & 0.06087500 \\ \mathrm{C} & -2.53469100 & -0.19116400 & 0.07154000 \\ \mathrm{C} & -3.07561800 & -1.47053700 & 0.37937600 \\ \mathrm{C} & -4.40935100 & -1.63447400 & 0.65608000 \\ \mathrm{H} & -3.61728300 & 3.05663200 & -0.24474200 \\ \mathrm{H} & -6.33989400 & -0.66822100 & 0.86297000 \\ \mathrm{H} & -5.46364500 & 1.58118100 & 0.34066500 \\ \mathrm{C} & -2.92702900 & 2.21702200 & -0.23977300 \\ \mathrm{C} & -1.14854500 & 0.02752200 & -0.22181900 \\ \mathrm{H} & -2.42921500 & -2.34166100 & 0.39803100 \\ \mathrm{H} & -4.79435800 & -2.62288700 & 0.88886200 \\ \mathrm{C} & -0.71329100 & 1.29940500 & -0.50434100 \\ \mathrm{C} & -1.59996600 & 2.39956200 & -0.51556600 \\ \mathrm{H} & -1.21663400 & 3.38977100 & -0.74298300 \\ \mathrm{C} & -0.18535100 & -1.15631800 & -0.27843400 \\ \mathrm{H} & -0.42899400 & -1.84316100 & 0.54209100 \\ \mathrm{C} & -0.33680000 & -1.92171100 & -1.61226800 \\ \mathrm{H} & 0.31554200 & -2.79969800 & -1.62847900 \\ \mathrm{H} & -1.37234100 & -2.24486900 & -1.75314000 \\ \mathrm{H} & -0.06436000 & -1.26898700 & -2.44653100 \\ \mathrm{H} & 0.33912000 & 1.46331800 & -0.71581000 \\ & & & \\ & & & \end{array}$

\section{References}

(S1) Hayashida, M.; Honda, H.; Hamana, M. Heterocycles 1990, 31, 1325.

(S2) Sun, H.-X.; Sun, Z.-H.; Wang, B. Tetrahedron Lett. 2009, 50, 1596.

(S3) Yazaki, R.; Kumagai, N.; Shibasaki, M. Org. Lett. 2011, 13, 952.

(S4) (a) Guin, J.; Varseev, G.; List, B. J. Am. Chem. Soc. 2013, 135, 2100. (b) Iwamoto, T.; Okuzono, C.; Adak, L.; Jin, M.; Nakamura, M. Chem.commun. 2019, 55, 1128.

(S5) (a) Fu, L.; Zhou, S.; Wan, X.; Chen, P.; Liu, G. J. Am. Chem. Soc. 2018, 140, 10965. (b) Liu, Y.; Xie, P.; Sun, Z.; Wo, X.; Gao, C.; Fu, W.; Loh, T.-P. Org. Lett. 2018, 20, 5353.

(S6) Frisch, M. J.; Trucks, G. W.; Schlegel, H. B.; Scuseria, G. E.; Robb, M. A.; Cheeseman, J. R.; Scalmani, G.; Barone, V.; Mennucci, B.; Petersson, G. A.; Nakatsuji, H.; Caricato, M.; 
Li, X.; Hratchian, H. P.; Izmaylov, A. F.; Bloino, J.; Zheng, G.; Sonnenberg, J. L.; Hada, M. E., M.; ; Toyota, K. F., R.; ; Hasegawa, J.; Ishida, M.; Nakajima, T.; Honda, Y.; Kitao, O.; Nakai, H.; Vreven, T.; Montgomery, J., J. A.;; Peralta, J. E.; Ogliaro, F.; Bearpark, M.; Heyd, J. J.; Brothers, E.; Kudin, K. N.; Staroverov, V. N.; Kobayashi, R.; Normand, J.; Raghavachari, K.; Rendell, A.; Burant, J. C.; Iyengar, S. S.; Tomasi, J.; Cossi, M.; Rega, N.; Millam, N. J.; Klene, M.; Knox, J. E.; Cross, J. B.; Bakken, V.; Adamo, C.; Jaramillo, J.; Gomperts, R.; Stratmann, R. E.; Yazyev, O.; Austin, A. J.; Cammi, R.; Pomelli, C.; Ochterski, J. W.; Martin, R. L.; Morokuma, K.; Zakrzewski, V. G.; Voth, G. A.; Salvador, P.; Dannenberg, J. J.; Dapprich, S.; Daniels, A. D.; Farkas, Ö.; Foresman, J. B.; Ortiz, J. V.; Cioslowski, J.; Fox, D. J., Gaussian 09, Gaussian, Inc., Wallingford CT, 2009.

(S7) Chai, J.-D.; Head-Gordon, M., Long-range corrected hybrid density functionals with damped atom-atom dispersion corrections. Phys. Chem. Chem. Phys. 2008, 10 (44), 66156620.

(S8) Dolg, M.; Wedig, U.; Stoll, H.; Preuss, H., Energy-adjusted ab initio pseudopotentials for the first row transition elements. The Journal of Chemical Physics 1987, 86 (2), 866-872. (S9) Ehlers, A.; Böhme, M.; Dapprich, S.; Gobbi, A.; Höllwarth, A.; Jonas, V.; Köhler, K.; Stegmann, R.; Veldkamp, A.; Frenking, G., A set of f-polarization functions for pseudopotential basis sets of the transition metals $\mathrm{Sc}-\mathrm{Cu}, \mathrm{Y}-\mathrm{Ag}$ and $\mathrm{La}-\mathrm{Au}$. Chemical physics letters 1993, 208 (1), 111-114.

(S10) Fukui, K., The path of chemical reactions-the IRC approach. Accounts of chemical research 1981, 14 (12), 363-368.

(S11) Weigend, F.; Ahlrichs, R., Balanced basis sets of split valence, triple zeta valence and quadruple zeta valence quality for $\mathrm{H}$ to $\mathrm{Rn}$ : Design and assessment of accuracy. Phys. Chem. Chem. Phys. 2005, 7 (18), 3297-3305.

(S12) Barone, V.; Cossi, M., Quantum calculation of molecular energies and energy gradients in solution by a conductor solvent model. J. Phys. Chem. A 1998, 102 (11), 1995-2001. (S13) Cossi, M.; Rega, N.; Scalmani, G.; Barone, V., Energies, structures, and electronic properties of molecules in solution with the C-PCM solvation model. J. Comput. Chem. 2003, 24 (6), 669-681. 